\title{
Genetically Intact Bioengineered Spores of Bacillus subtilis
}

Juan F. Quijano* and Ozgur Sahin

* = Corresponding author

\section{Supporting Information}

Supporting Tables 1-2: Supporting Table 1 with the primers used for building the plasmids in our study, and Supporting Table 2 with the primers used for recombination detection.

Supporting Figures 1-21: Supporting Figure 1 with spore germination experiments for detection of plasmid retention, Supporting Figures 2-19 with plasmid maps, Supporting Figure 20 with additional visualization of the GFP strain pCas9g2-D15-GFP, and Supporting Figure 21 with spore germination experiments of strains derived from plasmids that mutated.

Supporting DNA sequences: DNA sequences corresponding to the plasmids shown in Supporting Figures 2-19. 
Supporting Table 1. Primers used for the construction of the plasmids in our study

\begin{tabular}{|c|c|c|c|c|}
\hline Oligo & Oligo sequence $\left(5^{\prime} \rightarrow 3^{\prime}\right)$ & Target DNA & Source & $\begin{array}{l}\text { Resulting } \\
\text { plasmid }\end{array}$ \\
\hline 001 & ttgtccttttccgctgcata AACAATATGGCCCGTTTG & \multirow{2}{*}{ pHY300PLK } & \multirow{2}{*}{ Takara Bio - \#3060 } & \multirow{4}{*}{$\mathrm{pV} 1$} \\
\hline 002 & gccagggttttcccagtcac TGCAGATCTCTAGAAGCT & & & \\
\hline 003 & caagcttctagagatctgca GTGACTGGGAAAACCCT & \multirow{2}{*}{ pSEVA3b61 } & \multirow{2}{*}{ Addgene - \#58310 } & \\
\hline 004 & aacaaacgggccatattgtt TATGCAGCGGAAAAGGAC & & & \\
\hline 005 & tatggaaaaacgctttgccc & \multirow{3}{*}{ gB1 } & \multirow{3}{*}{$\begin{array}{l}\text { Gene fragment } \\
\text { synthesis }\end{array}$} & \multirow{7}{*}{$\mathrm{pV} 1 \mathrm{RX}$} \\
\hline 005 & CACACAGGCTCTTGAACAAG & & & \\
\hline 006 & AACACCATTGACTCCTTCATC & & & \\
\hline 007 & GCAGCCAAGTGAATACACTG & & & \\
\hline 008 & $\begin{array}{c}\text { Actgcagatctctagaagct } \\
\text { ATGCTGAATGACTTCTTACAGC }\end{array}$ & gB2 & synthesis & \\
\hline 009 & tgtaagaagtcattcagcat AGCTTCTAGAGATCTGCA & \multirow{2}{*}{$\mathrm{pV} 1$} & \multirow{2}{*}{ This study } & \\
\hline 010 & cttgttcaagagcctgtgtg GGGCAAAGCGTTTTTCC & & & \\
\hline 011 & tacggttatccacagaatca GAGGGCAAACTACTAGAGA & & & \multirow{5}{*}{$\mathrm{pBb}$} \\
\hline 012 & ccaagtaatcgtgaatgtcg & $\mathrm{pV} 1 \mathrm{RX}$ & This study & \\
\hline & ACCGGATAAGGAGATCATTG & \multirow{3}{*}{$\begin{array}{l}\text { pJWV102-PL- } \\
\text { dCas9 }\end{array}$} & & \\
\hline 013 & caatgatctccttatccggt CGACATTCACGATTACTTGG & & \multirow{2}{*}{ Addgene - \#85588 } & \\
\hline 014 & atctctagtagtttgccctc TGATTCTGTGGATAACCGTA & & & \\
\hline 015 & gcttgtcagggggcg GAGCCTATGGAAAAACGCTTTG & \multirow[b]{2}{*}{ gB20 } & \multirow[b]{2}{*}{$\begin{array}{l}\text { Gene fragment } \\
\text { synthesis }\end{array}$} & \multirow{6}{*}{ pV1-PsspA } \\
\hline 016 & $\begin{array}{c}\text { ccctttaagagcgtcatgta } \\
\text { AACTTCATTGTAAATTCTGAAGAAATACTTGTCAAG }\end{array}$ & & & \\
\hline 017 & $\begin{array}{c}\text { tcagaatttacaatgaagtt } \\
\text { TACATGACGCTCTTAAAGGGG }\end{array}$ & \multirow[t]{2}{*}{ gB20 } & \multirow{2}{*}{$\begin{array}{l}\text { Gene fragment } \\
\text { synthesis }\end{array}$} & \\
\hline 018 & tcgccagggttttc CCAGTCACTGCAGATCTCT & & & \\
\hline 019 & AGCTTCTAGAGATCTGCA & \multirow{2}{*}{$\mathrm{pV} 1$} & \multirow{2}{*}{ This study } & \\
\hline 020 & GGGCAAAGCGTTTTTCC & & & \\
\hline 021 & AATAGGCTTAGATATCGGCACA & \multirow{2}{*}{ pCas9 } & \multirow{2}{*}{ Addgene - \#42876 } & \multirow{4}{*}{$\begin{array}{l}\text { pCas9g1- } \\
\text { cons }\end{array}$} \\
\hline 022 & TCAGTCACCTCCTAGCTG & & & \\
\hline 023 & GAAACACGCATTGATTTTGAGT & \multirow{2}{*}{ pV1-PsspA } & \multirow{2}{*}{ This study } & \\
\hline 024 & CCCATCCGACGCTATTTGT & & & \\
\hline 025 & gactggagcttctagagatc AGGTCAGACGCGATTTCC & \multirow{3}{*}{ gB26 } & \multirow{3}{*}{$\begin{array}{l}\text { Gene fragment } \\
\text { synthesis }\end{array}$} & \multirow{4}{*}{$\begin{array}{l}\text { pCas9g2- } \\
\text { cons }\end{array}$} \\
\hline 026 & gggttttcccagtcactgca TGACCCAGACACCTATTCC & & & \\
\hline 027 & aggaataggtgtctgggtca TGCAGTGACTGGGAAAACC & & & \\
\hline 028 & $\begin{array}{c}\text { caggaaatcgcgtctgacct } \\
\text { GATCTCTAGAAGCTCCAGTCAC }\end{array}$ & pCas9g1-cons & This study & \\
\hline
\end{tabular}




\begin{tabular}{|c|c|c|c|c|}
\hline & & & & \\
\hline 029 & tacaatgaagtttacatgac & & & \\
\hline 029 & GATTAACTAATAAGGAGGGCAAAC & gB27 & Gene fragment & \\
\hline 030 & ctaaaaccccctttaagagc & & & \\
\hline & TTATCATTGTTCCGCGATCTC & & & pCas9g1- \\
\hline 031 & $\begin{array}{c}\text { agatcgcggaacaatgataa } \\
\text { GCTCTTAAAGGGGGTTTTAGA }\end{array}$ & & & D15-cons \\
\hline & & pCas9g1-cons & This study & \\
\hline 032 & $\begin{array}{l}\text { gccctccttattagttaatc } \\
\text { GTCATGTAAACTTCATTGTAAATTCTGAAG }\end{array}$ & & & \\
\hline 033 & tacaatgaagtttacatgac & & & \\
\hline 033 & GATTAACTAATAAGGAGGGCAAAC & gB27 & Gene fragment & \\
\hline 034 & ctaaaaccccctttaagagc & gD 21 & synthesis & \\
\hline 034 & TTATCATTGTTCCGCGATCTC & & & pCas9g2- \\
\hline 035 & agatcgcggaacaatgataa & & & D15-cons \\
\hline 035 & GCTCTTAAAGGGGGTTTTAAGA & & & \\
\hline 036 & $\begin{array}{c}\text { gccctccttattagttaatc } \\
\text { GTCATGTAAACTTCATTGTAAATTCTGAAG }\end{array}$ & pCas9g2-cons & This study & \\
\hline 037 & gttttggtccctcaatgatc TCCTTATCCGGTTAAGATGG & aB27 & Gene fragment & \\
\hline 038 & ttgtgccgatatcta AGCCTATTGAGTATTTCTTATCCAT & GD $\angle 1$ & synthesis & \\
\hline 039 & ATGGATAAGAAATACTCAATAGGCT & nCacor1-sonc & Thic ctudve & plasogd \\
\hline 040 & CCATCTTAACCGGATAAGGA & plassge-conss & IIIIs study & \\
\hline 041 & gttttggtccctcaatgatc TCCTTATCCGGTTAAGATGG & $\sigma R 27$ & Gene fragment & \\
\hline 042 & ttgtgccgatatcta AGCCTATTGAGTATTTCTTATCCAT & gBLI & synthesis & 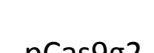 \\
\hline 043 & ATGGATAAGAAATACTCAATAGGCT & nCacof2_rons & This ctudy & plasoge \\
\hline 044 & CCATCTTAACCGGATAAGGA & $5 c a s z 8<-60113$ & 11115 stuny & \\
\hline 045 & gttttggtccctcaatgatc TCCTTATCCGGTTAAGATGG & $\sigma B 28$ & Gene fragment & \\
\hline 046 & cctcttcctcctcgatgaat TTGCCCCAGGATTTACTCAT & $g B<8$ & synthesis & pCas9g1- \\
\hline 047 & ATGAGTAAATCCTGGGGCAA & nCac9o1-D15_ranc & Thic ctudve & D15 \\
\hline 048 & CCATCTTAACCGGATAAGGA & 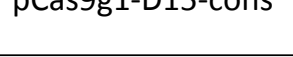 & IIIIs study & \\
\hline 049 & gttttggtccctcaatgatc TCCTTATCCGGTTAAGATGG & $\sigma \mathrm{B} 38$ & Gene fragment & \\
\hline 050 & cctcttcctcctcgatgaat TTGCCCCAGGATTTACTCAT & $g B<8$ & synthesis & pCas9g2- \\
\hline 051 & ATGAGTAAATCCTGGGGCAA & nCacas)-D15-ronc & This ctudy & D15 \\
\hline 052 & CCATCTTAACCGGATAAGGA & מוs & IIIIs study & \\
\hline 053 & ccttatccggttaagatggc GAGTAAATCCTGGGGCAAA & nCas9g1-D15 & This studv & pCas9g1- \\
\hline 054 & atttgccccaggatttactc GCCATCTTAACCGGATAAGG & plasygi-DiJ & Ihis study & D15-mut \\
\hline 055 & ccttatccggttaagatggc GAGTAAATCCTGGGGCAAA & $n$ racas)-D15 & This studv & pCas9g2- \\
\hline 056 & atttgccccaggatttactc GCCATCTTAACCGGATAAGG & 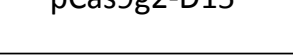 & 1111s study & D15-mut \\
\hline 057 & gagcgtctcagcttctagag GTGACTGGGAAAACCCTG & & & \\
\hline 058 & $\begin{array}{c}\text { cggataaggagatcattgag } \\
\text { GAAGCTATACTGTAAAGTACAAGCA }\end{array}$ & pCas9g1-D15 & This study & $\begin{array}{l}\text { pCas9g1- } \\
\text { D15-GFP }\end{array}$ \\
\hline 059 & gtactttacagtatagcttc CTCAATGATCTCCTTATCCGG & $\sigma B 2+2+2+$ & Gene fragment & \\
\hline 060 & gccagggttttcccagtcac CTCTAGAAGCTGAGACGCT & GD $\angle 1$ & synthesis & \\
\hline 061 & $\begin{array}{c}\text { gagcgtctcagcttctagag } \\
\text { TAGAGATCTGCAGTGACTGG }\end{array}$ & pCas9g2-D15 & This study & $\begin{array}{l}\text { pCas9g2- } \\
\text { D15-GFP }\end{array}$ \\
\hline
\end{tabular}




\begin{tabular}{|c|c|c|c|c|}
\hline 062 & $\begin{array}{c}\text { cggataaggagatcattgag } \\
\text { GAAGCTATACTGTAAAGTACAAGCA }\end{array}$ & & & \\
\hline $\begin{array}{l}063 \\
064\end{array}$ & $\begin{array}{l}\text { gtactttacagtatagcttc CTCAATGATCTCCTTATCCGG } \\
\text { ccagtcactgcagatctcta CTCTAGAAGCTGAGACGCT }\end{array}$ & gB21 & $\begin{array}{l}\text { Gene fragment } \\
\text { synthesis }\end{array}$ & \\
\hline 065 & $\begin{array}{c}\text { ttaagatggcaagcttgaca GCCGAATTCGGATTCCCG } \\
\text { ttgtaaagctcgtccatccc } \\
\text { CGGGCATGCTTATTTTTTTGGA }\end{array}$ & $\begin{array}{l}\text { B. subtilis MF2352 } \\
\text { chromosome }\end{array}$ & $\begin{array}{l}\text { Ref. } 30 \text { of the } \\
\text { manuscript }\end{array}$ & \multirow{2}{*}{$\begin{array}{l}\text { pCas9g1 } \\
\text { D15-KinA }\end{array}$} \\
\hline 067 & $\begin{array}{c}\text { caaaaaaataagcatgcccg GGGATGGACGAGCTTTAC } \\
\text { cgcgggaatccgaattcggc } \\
\text { TGTCAAGCTTGCCATCTTAAC }\end{array}$ & pCas9g1-D15-GFP & This study & \\
\hline $\begin{array}{l}069 \\
070\end{array}$ & $\begin{array}{c}\text { ttaagatggcaagcttgaca GCCGAATTCGGATTCCCG } \\
\text { ttgtaaagctcgtccatccc } \\
\text { CGGGCATGCTTATTTTTTTGGA }\end{array}$ & $\begin{array}{l}\text { B. subtilis MF2352 } \\
\text { chromosome }\end{array}$ & $\begin{array}{l}\text { Ref. } 30 \text { of the } \\
\text { manuscript }\end{array}$ & \multirow{2}{*}{$\begin{array}{l}\text { pCas9g2- } \\
\text { D15-KinA }\end{array}$} \\
\hline 071 & $\begin{array}{c}\text { caaaaaaataagcatgcccg GGGATGGACGAGCTTTAC } \\
\text { cgcgggaatccgaattcggc } \\
\text { TGTCAAGCTTGCCATCTTAAC }\end{array}$ & pCas9g2-D15-GFP & This study & \\
\hline
\end{tabular}


Supporting Table 2. Plasmid integration into the chromosome

\begin{tabular}{ll} 
Plasmid & Colonies with recombination \\
\hline pCas9g1-D15 & $0 / 100$ \\
pCas9g1-D15-GFP & $1 / 100$ \\
pCas9g1-D15-KinA & $1 / 100$ \\
pCas9g2-D15 & $1 / 100$ \\
pCas9g2-D15-GFP & $0 / 100$ \\
pCas9g2-D15-KinA & $2 / 100$
\end{tabular}


Supporting Table 3. Primers used for identification of plasmid integration events through reverse PCR

\begin{tabular}{|c|c|c|c|}
\hline Oligo & Target & Oligo sequence $\left(5^{\prime} \rightarrow 3^{\prime}\right)$ & Plasmid(s) \\
\hline R001 & \multirow{2}{*}{1} & CCTGAATAGAGTTCATAAACAATCC & \multirow{4}{*}{ All } \\
\hline R002 & & TTCAGGAATTGTCAGATAGGCC & \\
\hline R003 & \multirow{2}{*}{$1 \mathrm{~A}$} & AACAATCCTGCATGATAACCATC & \\
\hline R004 & & GTCAGATAGGCCTAATGACTG & \\
\hline R005 & \multirow{4}{*}{$2 \mathrm{~A}$} & TGCAGGGTAAAATTTATATCCTTCT & \multirow{4}{*}{ All } \\
\hline R006 & & TTAGTGACAAGGGTGATAAACTC & \\
\hline R007 & & ATATCCTTCTTGTTTTATGTTTCGG & \\
\hline R008 & & AGGGTGATAAACTCAAATACAGC & \\
\hline R009 & \multirow{4}{*}{$3 A$} & TACGGTGTAAACCTTCCTCCA & \multirow{4}{*}{ All } \\
\hline R010 & & TACACCGTAAAAGGTTAATCTCC & \\
\hline R011 & & CCTCCAAATCAGACAAACGTTTC & \\
\hline R012 & & GGTTAATCTCCTATGGTGGTTTG & \\
\hline R013 & \multirow{4}{*}{$4 \mathrm{~A}$} & CATTGCTCTCCTCCAGTTGC & \multirow{4}{*}{ All } \\
\hline R014 & & AATGAAACATGGCATTCAGTCAC & \\
\hline R015 & & CAGTTGCACATTGGACAAAGC & \\
\hline R016 & & GTCACAAAAGGTTGTTGCTGAAG & \\
\hline R017 & \multirow{4}{*}{$5 A$} & CCTCTCGGTTATGAGTTAGTTC & \multirow{4}{*}{ All } \\
\hline R018 & & AAGAACGAAGTCGAGATCAGG & \\
\hline R019 & & GTTATGAGTTAGTTCAAATTCGTTC & \\
\hline R020 & & GTCGAGATCAGGGAATGAGTT & \\
\hline R021 & \multirow{4}{*}{$6 \mathrm{~A}$} & AACTCGGTCGCCGCATACA & \multirow{4}{*}{ All } \\
\hline R022 & & TTGCCCGGCGTCAACACG & \\
\hline R023 & & CCGCATACACTATTCTCAGAATG & \\
\hline R024 & & ACACGGGATAATACCGCGC & \\
\hline R025 & \multirow{2}{*}{7} & TAGTTATCTACACGACGGGGA & \multirow{4}{*}{ All } \\
\hline R026 & & ACGATACGGGAGGGCTTAC & \\
\hline R027 & \multirow{2}{*}{$7 \mathrm{~A}$} & AGTCAGGCAACTATGGATGAAC & \\
\hline R028 & & CCATCTGGCCCCAGTGCT & \\
\hline R029 & \multirow{2}{*}{8} & GGATAAGGCGCAGCGGTC & \multirow{4}{*}{ All } \\
\hline R030 & & GGTAACTATCGTCTTGAGTCC & \\
\hline R031 & \multirow{2}{*}{$8 \mathrm{~A}$} & AGCGGTCGGACTGAACGG & \\
\hline R032 & & GTCCAACCCGGAAAGACATG & \\
\hline R033 & \multirow{2}{*}{9} & AGCTATGAGATGGAGAAAGCC & \multirow{2}{*}{ All } \\
\hline R034 & & TACCCAGGCTTAACCTTCTTC & \\
\hline
\end{tabular}




\begin{tabular}{|c|c|c|c|}
\hline $\begin{array}{l}\text { R035 } \\
\text { R036 }\end{array}$ & $9 A$ & $\begin{array}{c}\text { GCCATAACCATGAGTGATAACAC } \\
\text { CTTCTTCGGCTTTTTCACGAG }\end{array}$ & \\
\hline R037 & \multirow{2}{*}{10} & GCGTGTTCAGACTAGAACATC & \multirow{4}{*}{ All } \\
\hline R038 & & СССTTATCACCTAGAACAATCGT & \\
\hline R039 & \multirow{2}{*}{$10 \mathrm{~A}$} & ACCAGAGTATAAAGGTAATCGTG & \\
\hline R040 & & ATCGTAGTTCTGGCAGAGTAG & \\
\hline R041 & \multirow{2}{*}{11} & TGCTATTGCTGCTGTAGGTCA & \multirow{4}{*}{ All } \\
\hline R042 & & CCACACAGTAGGTAGGTAAATC & \\
\hline R043 & \multirow{2}{*}{$11 \mathrm{~A}$} & CAAGATGTGTTAGATAAGTTCACG & \\
\hline $\mathrm{R} 044$ & & ATCAACCAGAATCAAGTTTCGGA & \\
\hline R045 & \multirow{2}{*}{12} & GATTGAGGGAGATTTAAATCCTG & \multirow{4}{*}{ All } \\
\hline R046 & & CCAAATAGATTAAGCGCAAATCC & \\
\hline R047 & \multirow{2}{*}{$12 \mathrm{~A}$} & TCCTGATAATAGTGATGTGGACA & \\
\hline R048 & & CCGCTTTATCAGTAGAATCTACC & \\
\hline R049 & \multirow{4}{*}{$13 \mathrm{~A}$} & TTATATTGATGGGGGAGCTAGC & \multirow{4}{*}{ All } \\
\hline R050 & & CATCAATATAACCTGCATATCCG & \\
\hline R051 & & GGGGAGCTAGCCAAGAAGA & \\
\hline R052 & & CCTGCATATCCGTTTTTTGATTG & \\
\hline R053 & \multirow{4}{*}{$14 \mathrm{~A}$} & CCAGCATTTCTTTCAGGTGAAC & \multirow{4}{*}{ All } \\
\hline R054 & & GGTTTTCGCATTCCTTCAGTAAC & \\
\hline R055 & & GAACAGAAGAAAGCCATTGTTGA & \\
\hline R056 & & AACATATTTGACCTTTGTCAATTCG & \\
\hline R057 & \multirow{2}{*}{15} & GTCAAAGTAATGGGGCGGCA & \multirow{4}{*}{ All } \\
\hline R058 & & TGTTCATGTAAACTATCGCCTTG & \\
\hline R059 & \multirow{2}{*}{$15 \mathrm{~A}$} & CGGCATAAGCCAGAAAATATCG & \\
\hline R060 & & CCTTGTCCAGACACTTGTGC & \\
\hline R061 & \multirow{4}{*}{$16 \mathrm{~A}$} & GGTTGAAACTCGCCAAATCAC & \multirow{4}{*}{ All } \\
\hline R062 & & AATTGGCGTTTGATAAAACCAGC & \\
\hline R063 & & AAATCACTAAGCATGTGGCACAA & \\
\hline R064 & & GCTTTATCAAGTTCACTCAAACC & \\
\hline R065 & \multirow{2}{*}{17} & CACAGTGCGCAAAGTATTGTC & \multirow{4}{*}{ All } \\
\hline R066 & & CTGTGGCAAAATCTCGCCC & \\
\hline R067 & \multirow{2}{*}{$17 \mathrm{~A}$} & TCCATGCCCCAAGTCAATATTG & \\
\hline R068 & & CTCGCCCTTTATCCCAGACA & \\
\hline \multirow{2}{*}{ R069 } & & GCTAGTCATTATGAAAAGTTGAAG & \multirow{3}{*}{ All } \\
\hline & 18 & G & \\
\hline R070 & & CACATATTTGCTTGGCAGAGC & \\
\hline
\end{tabular}




\begin{tabular}{|c|c|c|c|}
\hline $\begin{array}{l}\text { R071 } \\
\text { R072 }\end{array}$ & $18 \mathrm{~A}$ & $\begin{array}{l}\text { GTTGAAGGGTAGTCCAGAAGA } \\
\text { GCAGAGCCAGCTCATTTCC }\end{array}$ & \\
\hline R073 & 10 & GAGATCTGCAGTGACTGGGA & \multirow{4}{*}{ pCas9g1-D15 } \\
\hline R074 & 10 & ATCTCTAGAAGCTCCAGTCAC & \\
\hline R075 & \multirow{2}{*}{$19 A$} & CCTGGCGACTAGTCTTGGA & \\
\hline R076 & & TCACTGCAGATCTCTAGAAGC & \\
\hline R077 & \multirow{2}{*}{20} & GAGATCAGGTCAGACGCGAT & \multirow{4}{*}{ pCas9g2-D15 } \\
\hline R078 & & ATCTCTAGAAGCTCCAGTCAC & \\
\hline R079 & \multirow{2}{*}{$20 A$} & GATTTCCTGGGTGCTCGAG & \\
\hline R080 & & TCACTGCAGATCTCTAGAAGC & \\
\hline R081 & \multirow{2}{*}{21} & CTCCATCTGGATTTGTTCAGAAC & \multirow{4}{*}{$\begin{array}{l}\text { pCas9g2-D, pCas9g2-D15- } \\
\text { GFP, pCas9g2-D15-KinA }\end{array}$} \\
\hline R082 & & AGTTCTGAGGTCATTACTGGATC & \\
\hline R083 & \multirow{2}{*}{$21 \mathrm{~A}$} & AGAACGCTCGGTTGCCGC & \\
\hline R084 & & TGGATCTATCAACAGGAGTCC & \\
\hline R085 & & GCAAGCTTGACAAGTATTTCTTC & \multirow{4}{*}{$\begin{array}{c}\text { pCas9g1-D15-GFP, pCas9g2- } \\
\text { D15-GFP }\end{array}$} \\
\hline R086 & $\angle 2$ & TGCCATCTTAACCGGATAAGG & \\
\hline R087 & \multirow{2}{*}{$22 \mathrm{~A}$} & GGAGGGCAAACTACTAGAGATG & \\
\hline R088 & & GATAAGGAGATCATTGAGGAAGC & \\
\hline R089 & \multirow{2}{*}{23} & CGTGCTTGAGGCGGACGA & \multirow{4}{*}{$\begin{array}{c}\text { pCas9g1-D15-GFP, pCas9g2- } \\
\text { D15-GFP }\end{array}$} \\
\hline R090 & & CACCGGTGTTTGTTCCTCGA & \\
\hline R091 & \multirow{2}{*}{$23 \mathrm{~A}$} & ACGACCTGTCAGAAGTGTTCA & \\
\hline R092 & & CGATCTTAAGGTCCGGTTCG & \\
\hline R093 & \multirow{2}{*}{24} & GCAGTAGCGACTACCAGAG & \multirow{4}{*}{$\begin{array}{c}\text { pCas9g1-D15-GFP, pCas9g2- } \\
\text { D15-GFP }\end{array}$} \\
\hline R094 & & TGAGCTTTTGCTGCTCTGGTA & \\
\hline R095 & \multirow{2}{*}{$34 \mathrm{~A}$} & ACCAGAGTAGTCGTAGCCC & \\
\hline R096 & & GGTAGTCGCTACTCTTCAGG & \\
\hline R097 & \multirow{2}{*}{25} & GGAGGACGGAAACATTCTGG & \multirow{4}{*}{$\begin{array}{c}\text { pCas9g1-D15-GFP, pCas9g2 } \\
\text { D15-GFP }\end{array}$} \\
\hline R098 & & TCGATTCCTTTGAGTTCAATACG & \\
\hline R099 & \multirow{2}{*}{$25 \mathrm{~A}$} & TTCTGGGCCACAAACTGGAAT & \\
\hline R100 & & TACGGTTTACCAGGGTGTCG & \\
\hline R101 & \multirow{2}{*}{26} & TTGGTGAGAATCCAGGGGTC & \multirow{4}{*}{ pCas9g1-D15-GFP } \\
\hline R102 & & GTTCTGAACAAATCCAGATGGAG & \\
\hline R103 & \multirow{2}{*}{$26 A$} & GGGGTCCCCAATAATTACGAT & \\
\hline R104 & & GGAGTTCTGAGGTCATTACTG & \\
\hline R105 & \multirow{2}{*}{27} & CGCCGAATGAAGCGGATGA & \\
\hline R106 & & TCCACCACCGCTTTCCCAT & pCas9g2-D15-GFP \\
\hline R107 & $27 \mathrm{~A}$ & ATGAAATTGCTCATCGTCTCGTG & \\
\hline
\end{tabular}




\begin{tabular}{|c|c|c|c|}
\hline $\mathrm{R} 108$ & & ATGGCTCGAGCACCCAGG & \\
\hline R109 & \multirow{2}{*}{28} & GACAGCCGAATTCGGATTCC & \multirow{4}{*}{$\begin{array}{c}\text { pCas9g1-D15-KinA, pCas9g2- } \\
\text { D15-KinA }\end{array}$} \\
\hline R110 & & AAGCTTGCCATCTTAACCGGA & \\
\hline R111 & \multirow{2}{*}{$28 \mathrm{~A}$} & GGATTCCCGCGTATATCTTGA & \\
\hline $\mathrm{R} 112$ & & GATAAGGAGATCATTGAGGAAGC & \\
\hline R113 & \multirow{2}{*}{29} & GAACCTGCAAGCCCGGAAT & \multirow{4}{*}{$\begin{array}{c}\text { pCas9g1-D15-KinA, pCas9g2- } \\
\text { D15-KinA }\end{array}$} \\
\hline R114 & & TCGCAGTTTAGGGATTGATGG & \\
\hline $\mathrm{R} 115$ & \multirow{2}{*}{$29 A$} & GGAATCGACTACATATATAACGG & \\
\hline R116 & & GATGGCCTGTTTCTTCTTCAAG & \\
\hline $\mathrm{R} 117$ & \multirow{2}{*}{30} & TTGTCAAGCAATCCTGGTTCAC & \multirow{4}{*}{$\begin{array}{c}\text { pCas9g1-D15-KinA, pCas9g2- } \\
\text { D15-KinA }\end{array}$} \\
\hline R118 & & TCTCTTTTACATCCTCGTGATCG & \\
\hline R119 & \multirow{2}{*}{$30 A$} & CCTTTCAGAACAGGGTCATCTA & \\
\hline R120 & & TCGCAAGGATGCAGCTGATC & \\
\hline R121 & \multirow{2}{*}{31} & ACCGAAGATGAGCATTCTGTTC & \multirow{4}{*}{$\begin{array}{c}\text { pCas9g1-D15-KinA, pCas9g2- } \\
\text { D15-KinA }\end{array}$} \\
\hline R122 & & TATGATAATGTCTACTGTTCCCC & \\
\hline $\mathrm{R} 123$ & \multirow{2}{*}{$31 \mathrm{~A}$} & GTTCATGTTACTGTCAAAGACGA & \\
\hline R124 & & CCCATCAGGCATTGATTCAAC & \\
\hline $\mathrm{R} 125$ & 32 & ACATGTGGACAGCCATCCTG & \multirow{4}{*}{$\begin{array}{c}\text { pCas9g1-D15-KinA, pCas9g2- } \\
\text { D15-KinA }\end{array}$} \\
\hline $\mathrm{R} 126$ & 32 & ATGTATAACTCCCTGATGGTTTTC & \\
\hline R127 & \multirow{2}{*}{$32 \mathrm{~A}$} & ATCCTGAAAAAGGCACAGCGT & \\
\hline $\mathrm{R} 128$ & & TGTCACCATTAATCCAAGCCC & \\
\hline RR129 & \multirow{2}{*}{33} & GTCATAGCTGTTTCCTGTGTG & \multirow{4}{*}{ pUC57 } \\
\hline RR130 & & CAAGCTTGGCGTAATCATGGT & \\
\hline RR131 & \multirow{2}{*}{$33 \mathrm{~A}$} & TGTGTGAAATTGTTATCCGCTCA & \\
\hline RR132 & & TGCAGAGGCCTGCATGCAA & \\
\hline RR133 & \multirow{2}{*}{34} & CCGCTTACCGGATACCTGT & \multirow{4}{*}{ pUC57 } \\
\hline RR134 & & GAAGCTCCCTCGTGCGCT & \\
\hline RR135 & \multirow{2}{*}{$34 \mathrm{~A}$} & TGTCCGCCTTTCTCCCTTC & \\
\hline RR136 & & CAGGCGTTTCCCCCTGGA & \\
\hline RR137 & \multirow{2}{*}{35} & GAACGAAAACTCACGTTAAGGG & \multirow{4}{*}{ pUC57 } \\
\hline RR138 & & ACGGGGTCTGACGCTCAG & \\
\hline RR139 & \multirow{2}{*}{$35 \mathrm{~A}$} & GGGATTTTGGTCATGAGATTATC & \\
\hline RR140 & & GGATCTCAAGAAGATCCTTTGAT & \\
\hline RR141 & \multirow{2}{*}{36} & AGCGGTTAGCTCCTTCGGT & \multirow{4}{*}{ pUC57 } \\
\hline RR142 & & CCCAACGATCAAGGCGAGTT & \\
\hline RR143 & \multirow{2}{*}{$36 \mathrm{~A}$} & GGTCCTCCGATCGTTGTCA & \\
\hline RR144 & & GGCTTCATTCAGCTCCGGTT & \\
\hline
\end{tabular}




\begin{tabular}{|c|c|c|c|}
\hline RR145 & 27 & САCCTGACGTCTAAGAAACCAT & \multirow{4}{*}{ pUC57 } \\
\hline RR146 & 3 & ACATTTCCCCGAAAAGTGCCA & \\
\hline RR147 & \multirow{2}{*}{$37 A$} & AGGCGTATCACGAGGCCC & \\
\hline RR148 & & TAGGGGTTCCGCGCACATTT & \\
\hline RR149 & \multirow{2}{*}{38} & GAAAGGGGGATGTGCTGCAA & \multirow{4}{*}{ pUC57 } \\
\hline RR150 & & CTTCGCTATTACGCCAGCTG & \\
\hline RR151 & \multirow{2}{*}{$38 \mathrm{~A}$} & TCGGTGCGGGCCTCTTC & \\
\hline RR152 & & GCAAGGCGATTAAGTTGGGTA & \\
\hline
\end{tabular}

Targets labeled with an "A" (1A, 2A, 3A...) correspond to nested PCRs, which are performed when increased specificity is needed 
Supporting Figure 1. Germination of spores in LB or Valine media, and growth in LB-agar with or without antibiotic. $\mathrm{Cm}=$ chloramphenicol.
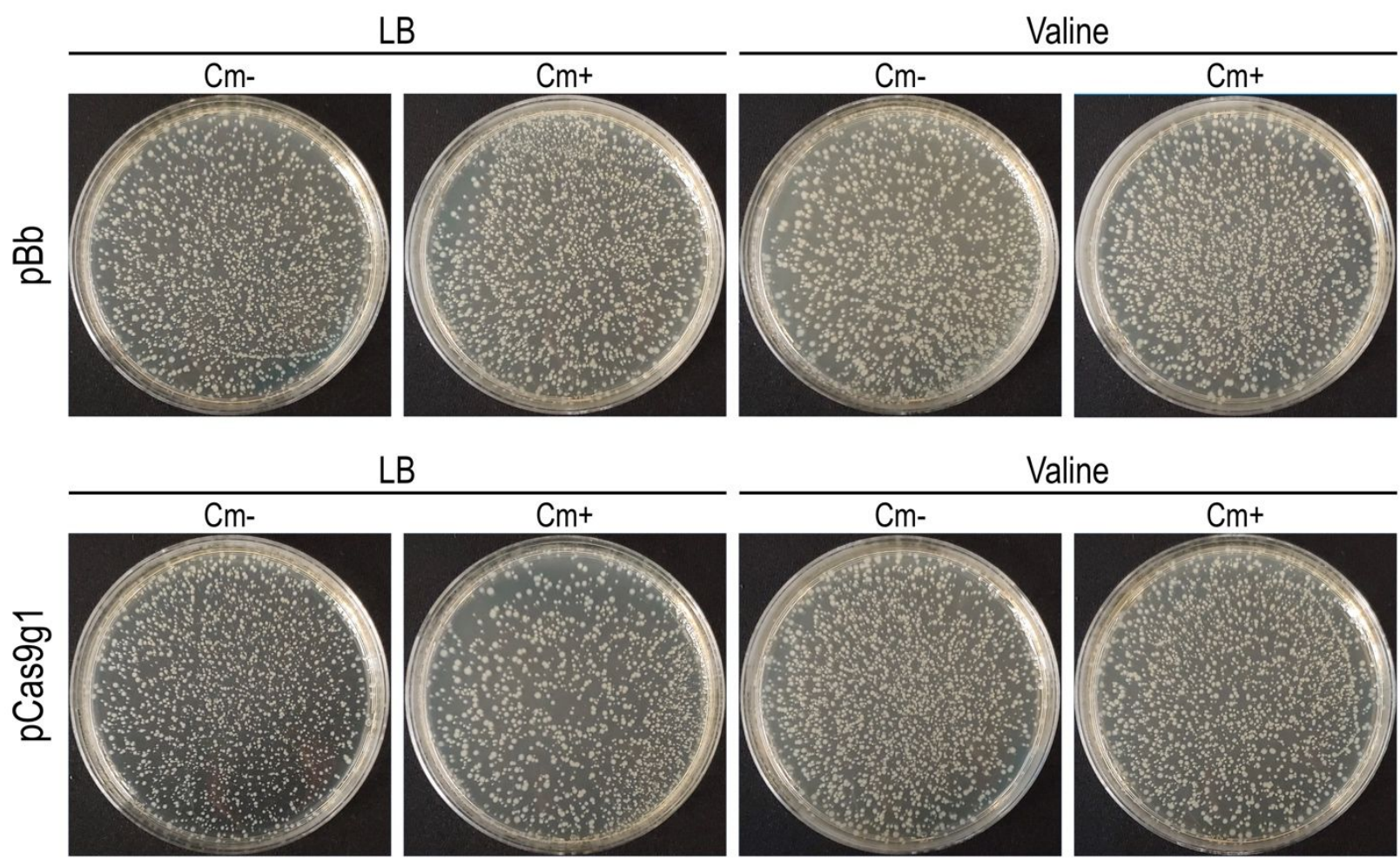

B

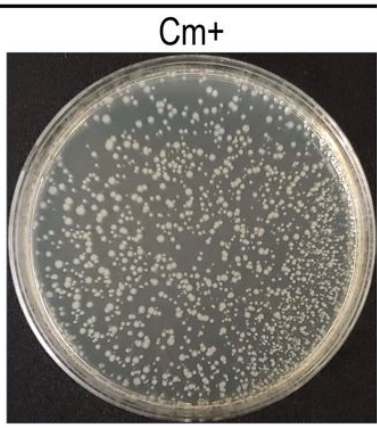

Valine

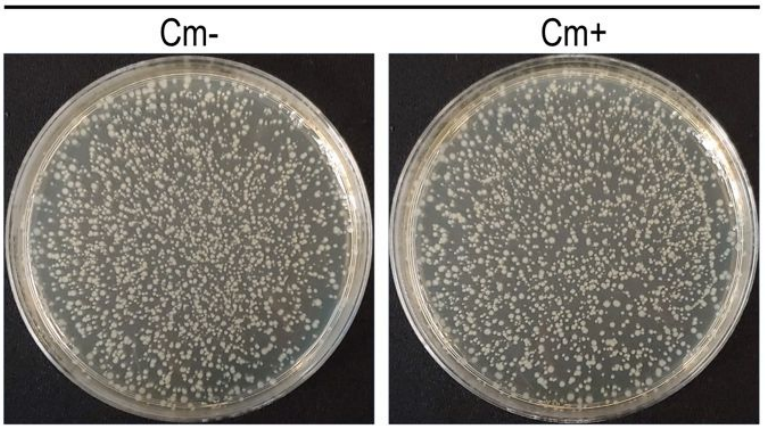

LB
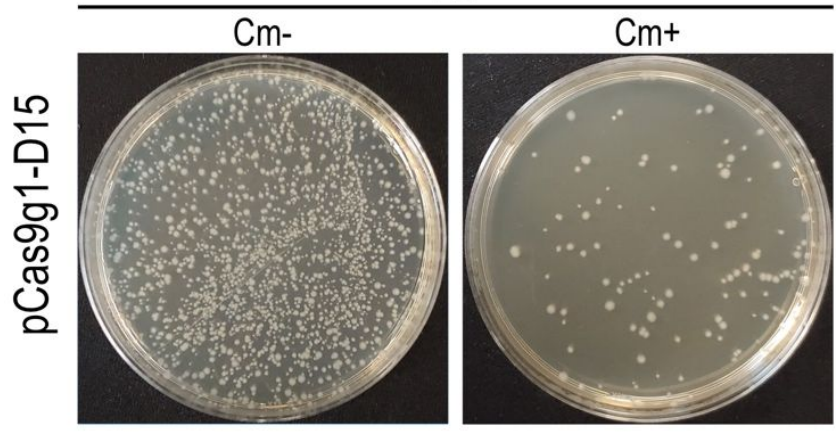

Valine
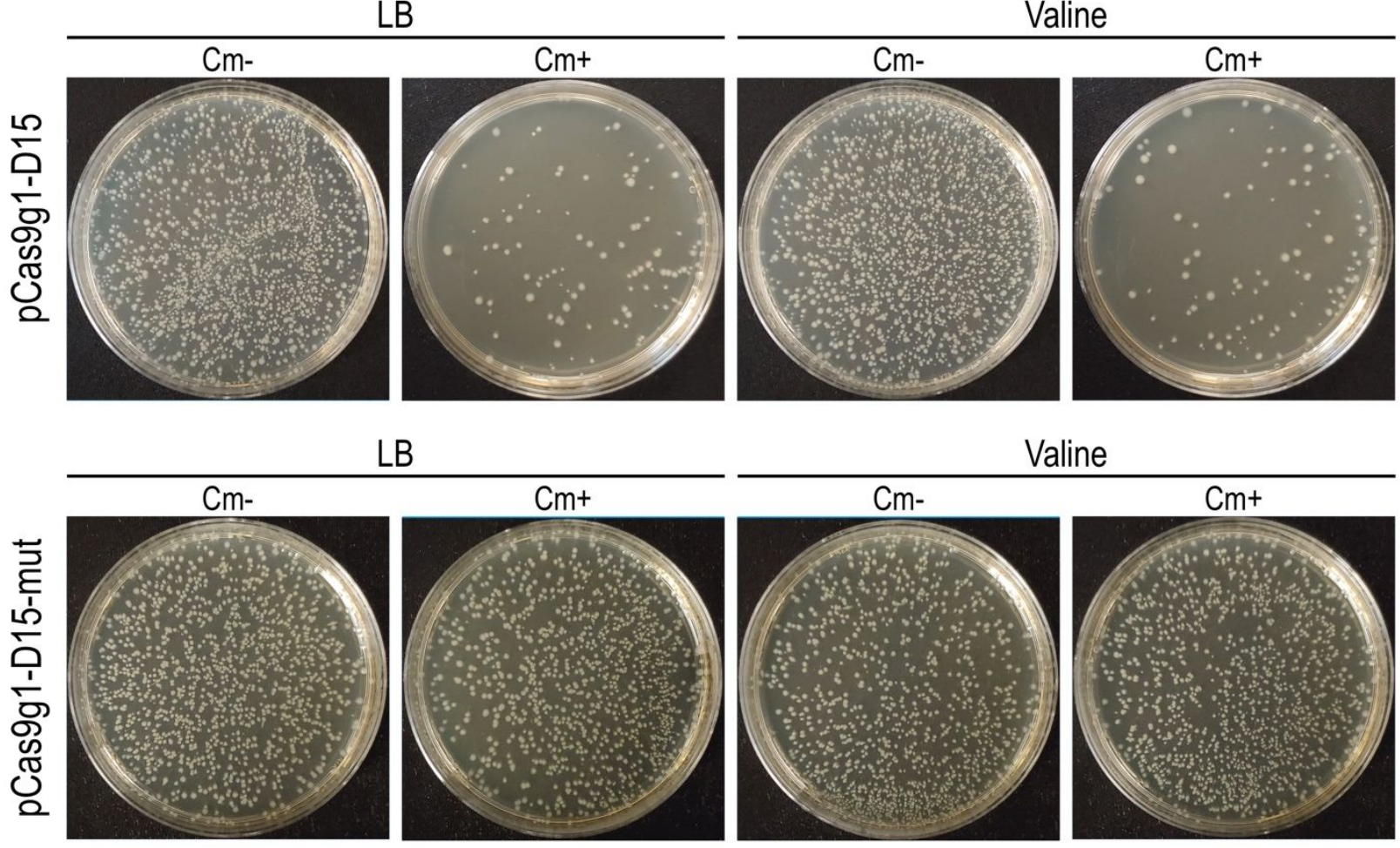

B

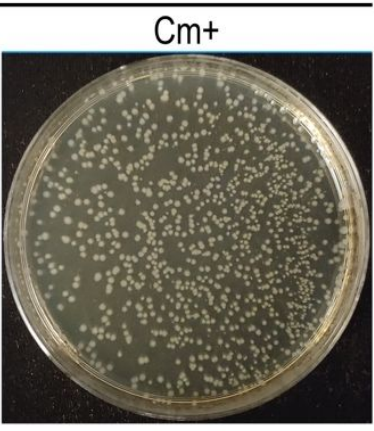

Valine
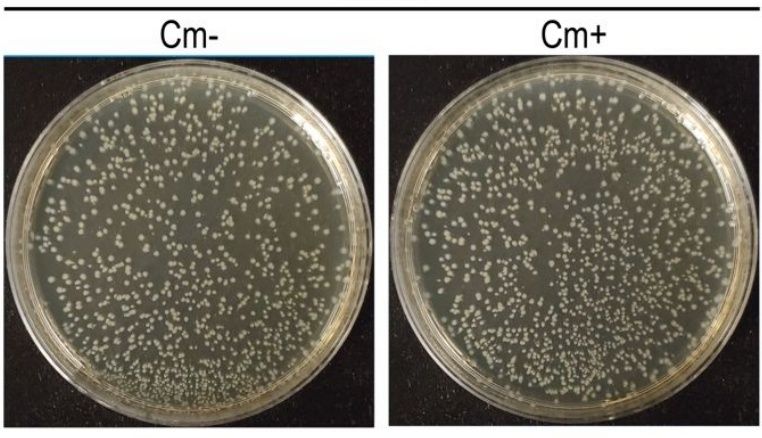
... continuation of Supporting Figure 1
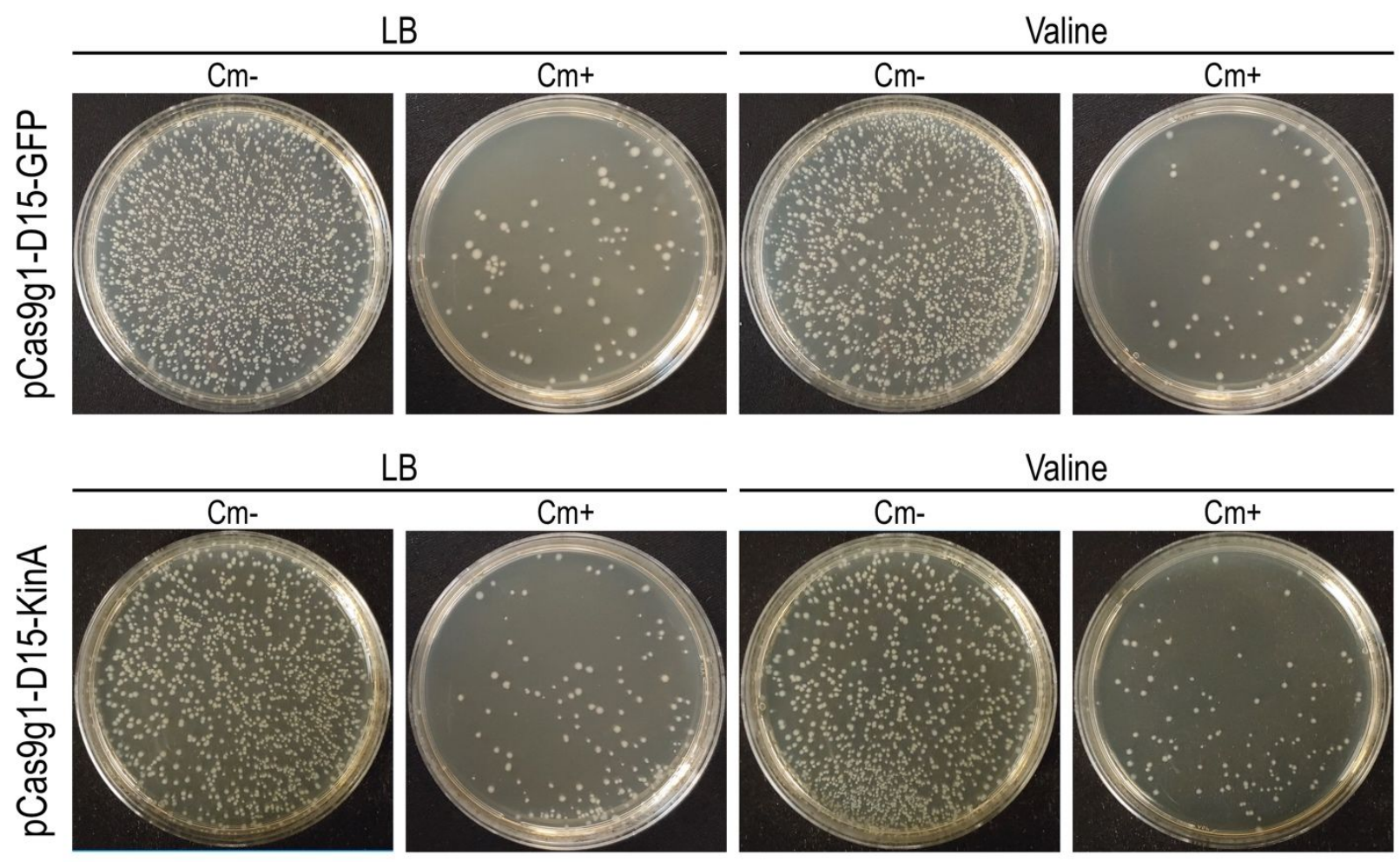

$B$

Valine
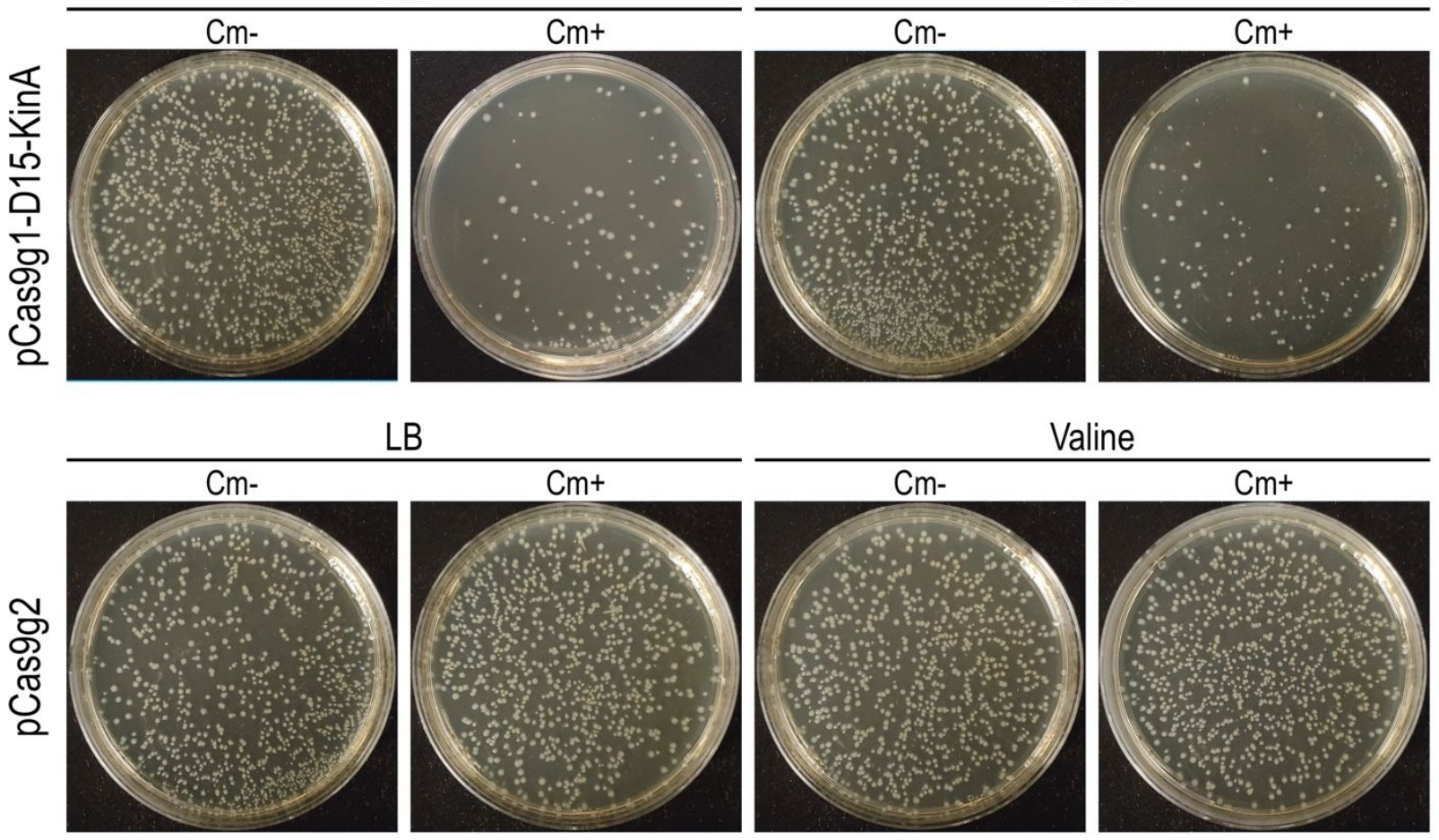

LB

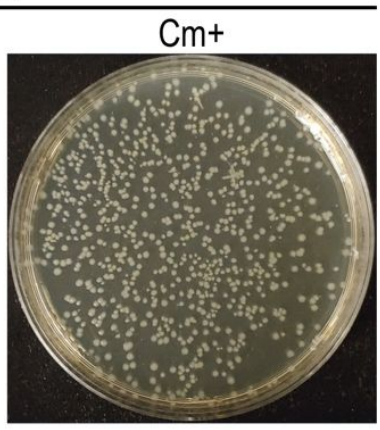

Valine
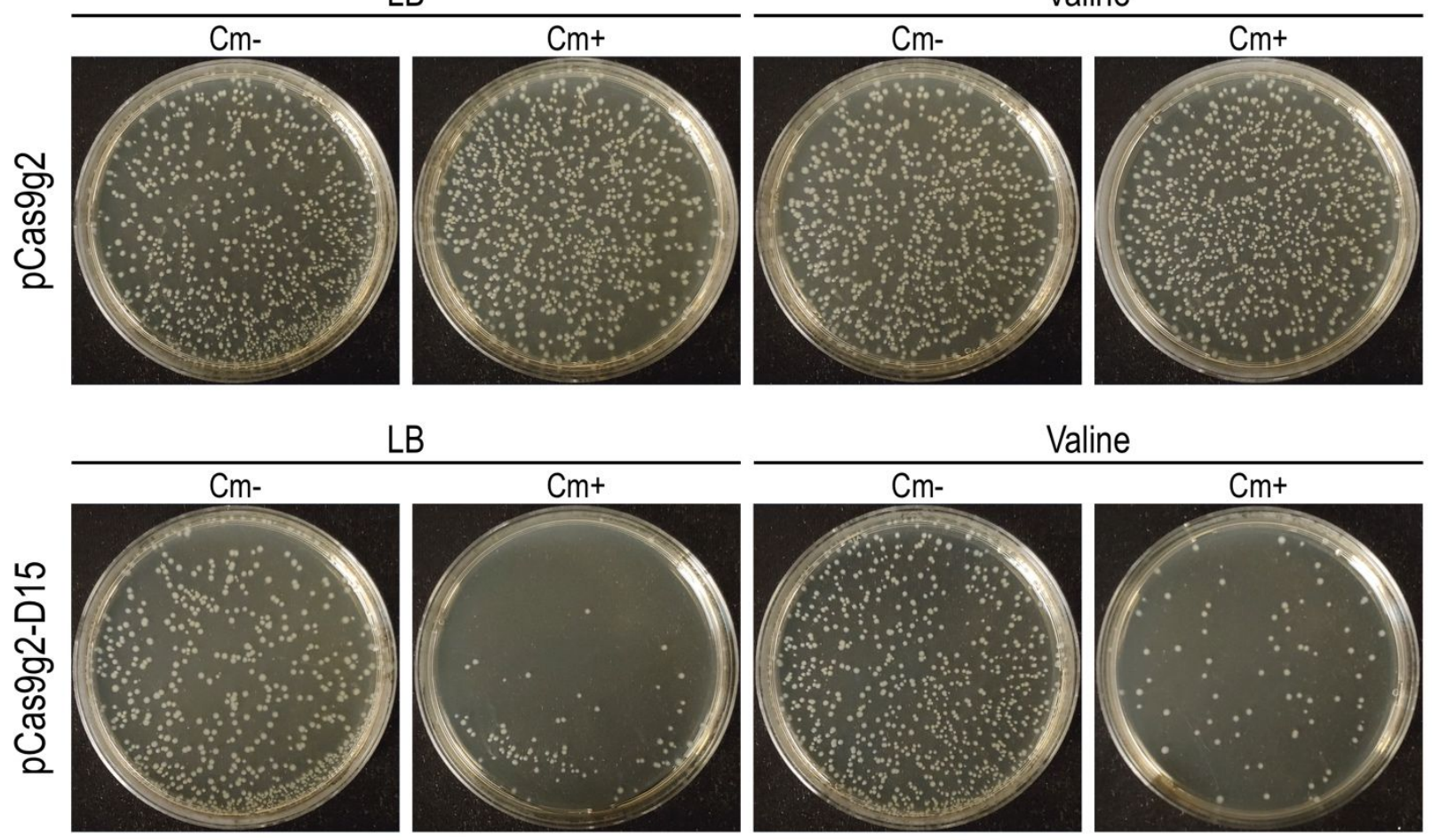

LB

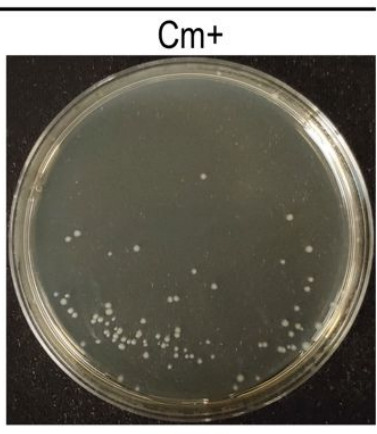

Valine

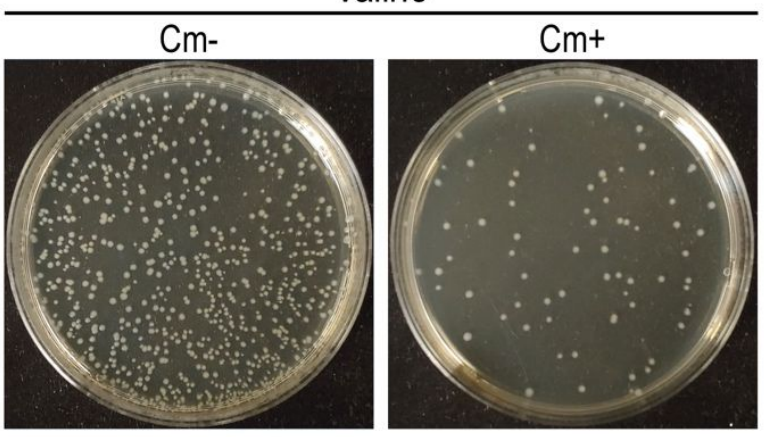


... continuation of Supporting Figure 1
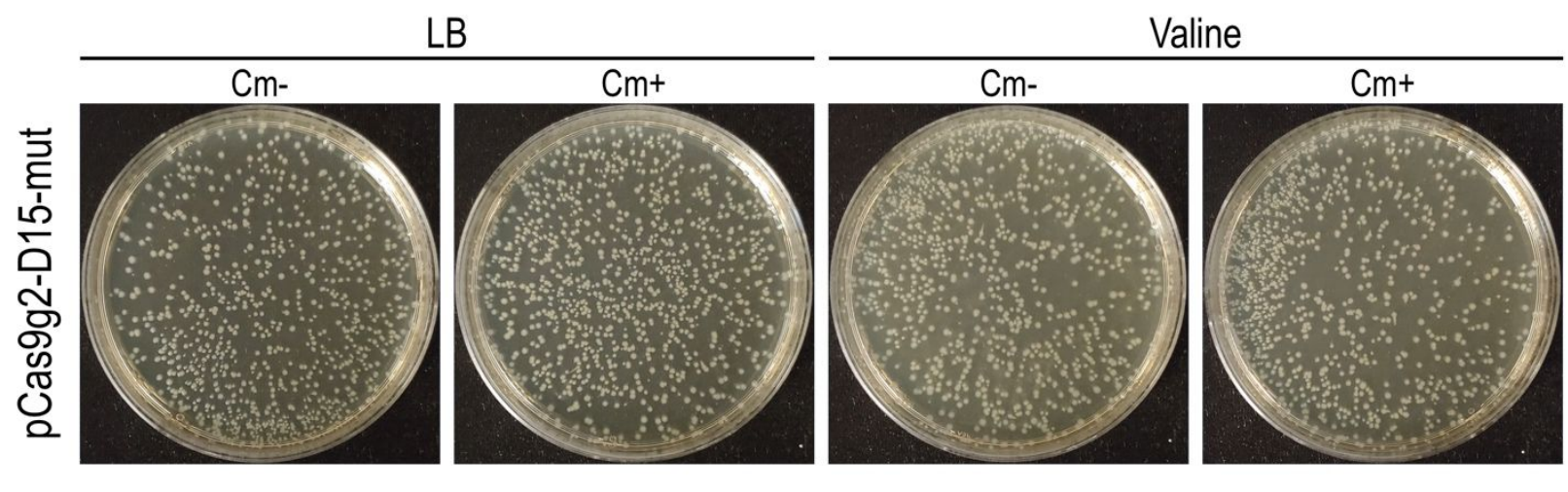

LB
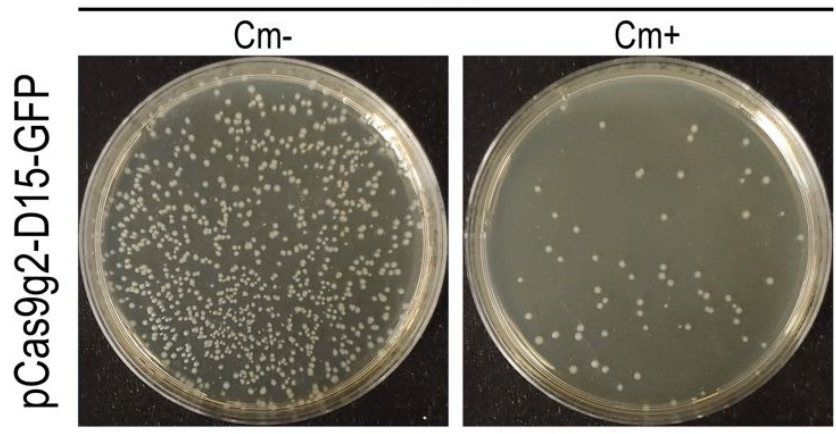

Valine

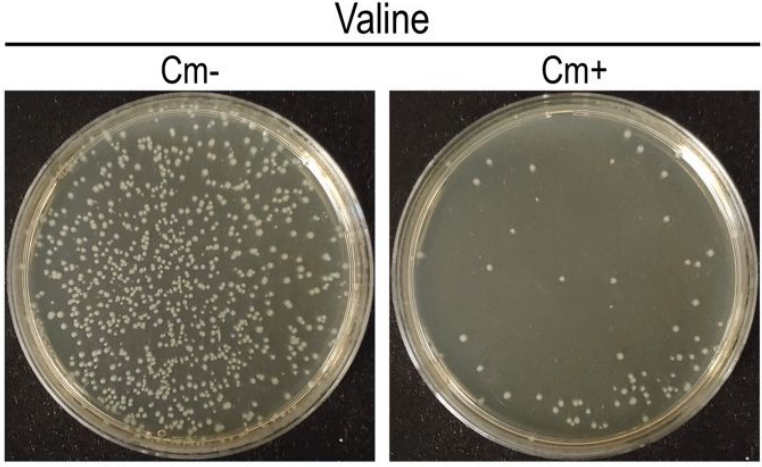

LB

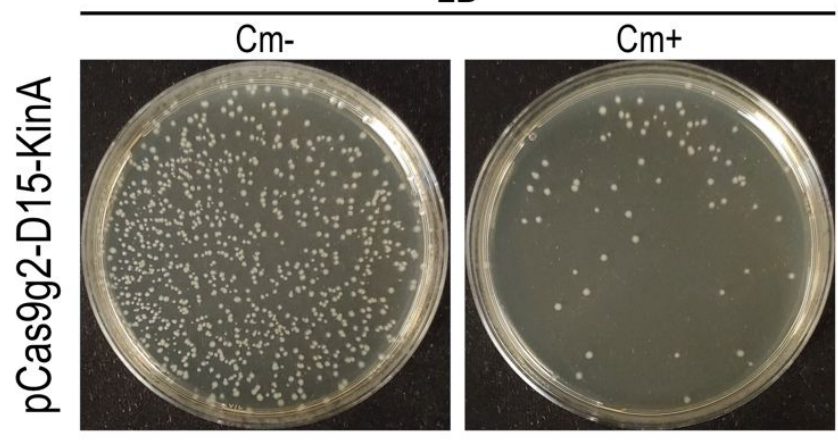

Valine
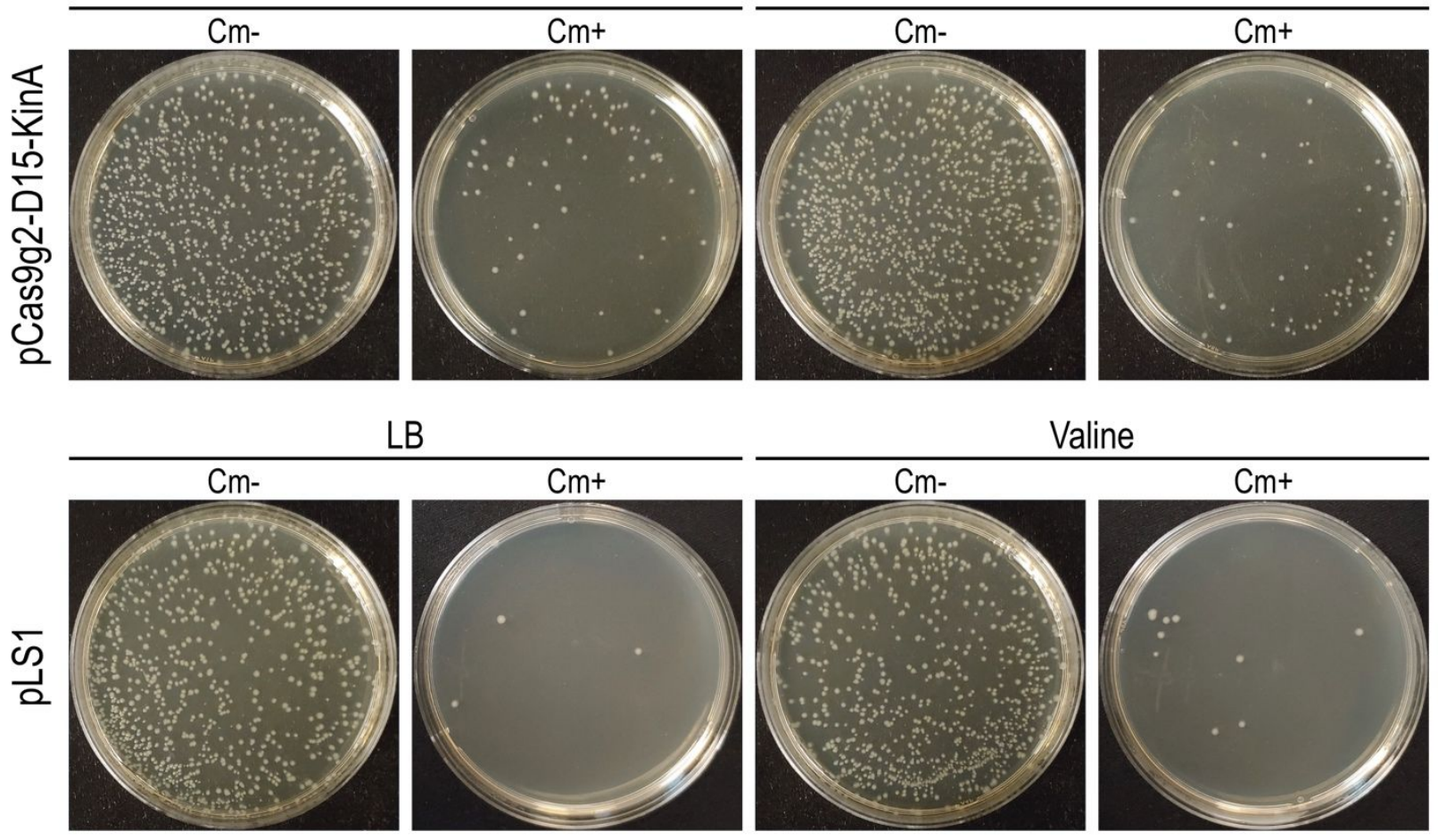

LB

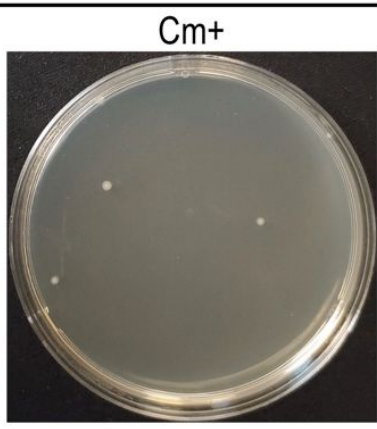

Valine

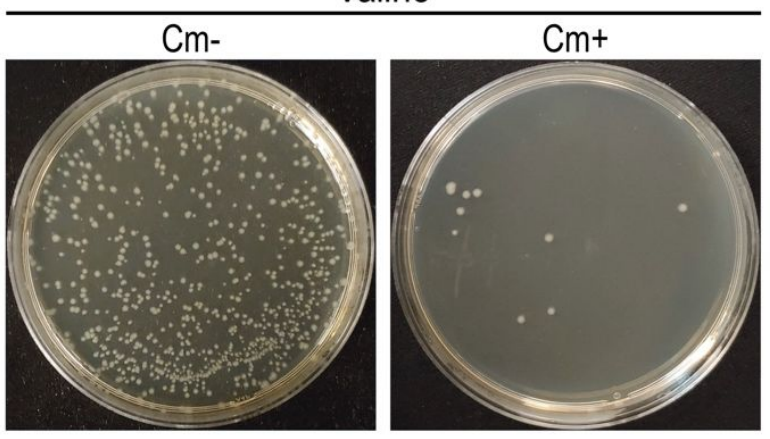


Supporting Figure 2. Map of plasmid pV1.

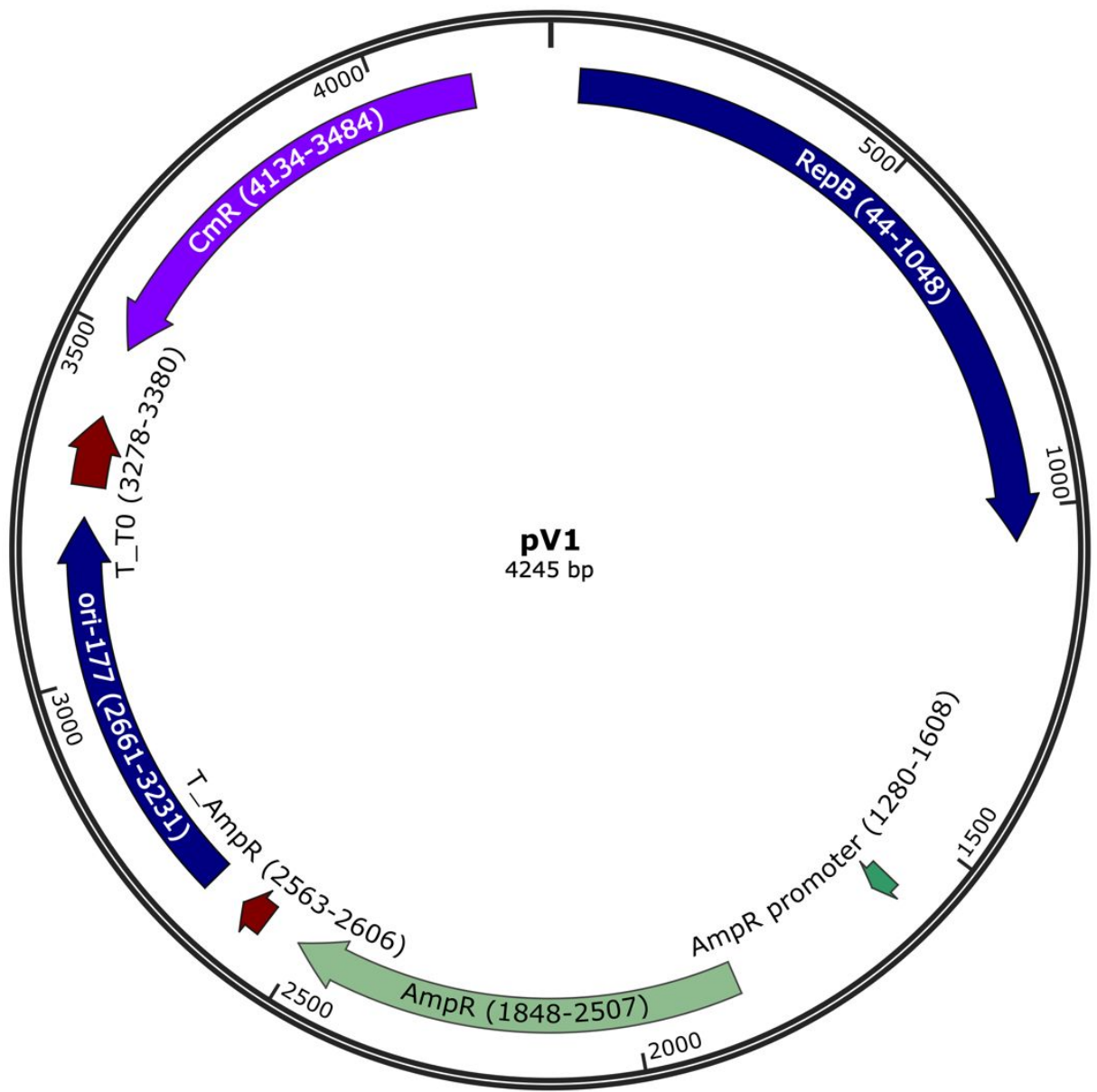


Supporting Figure 3. Map of plasmid pV1RX.

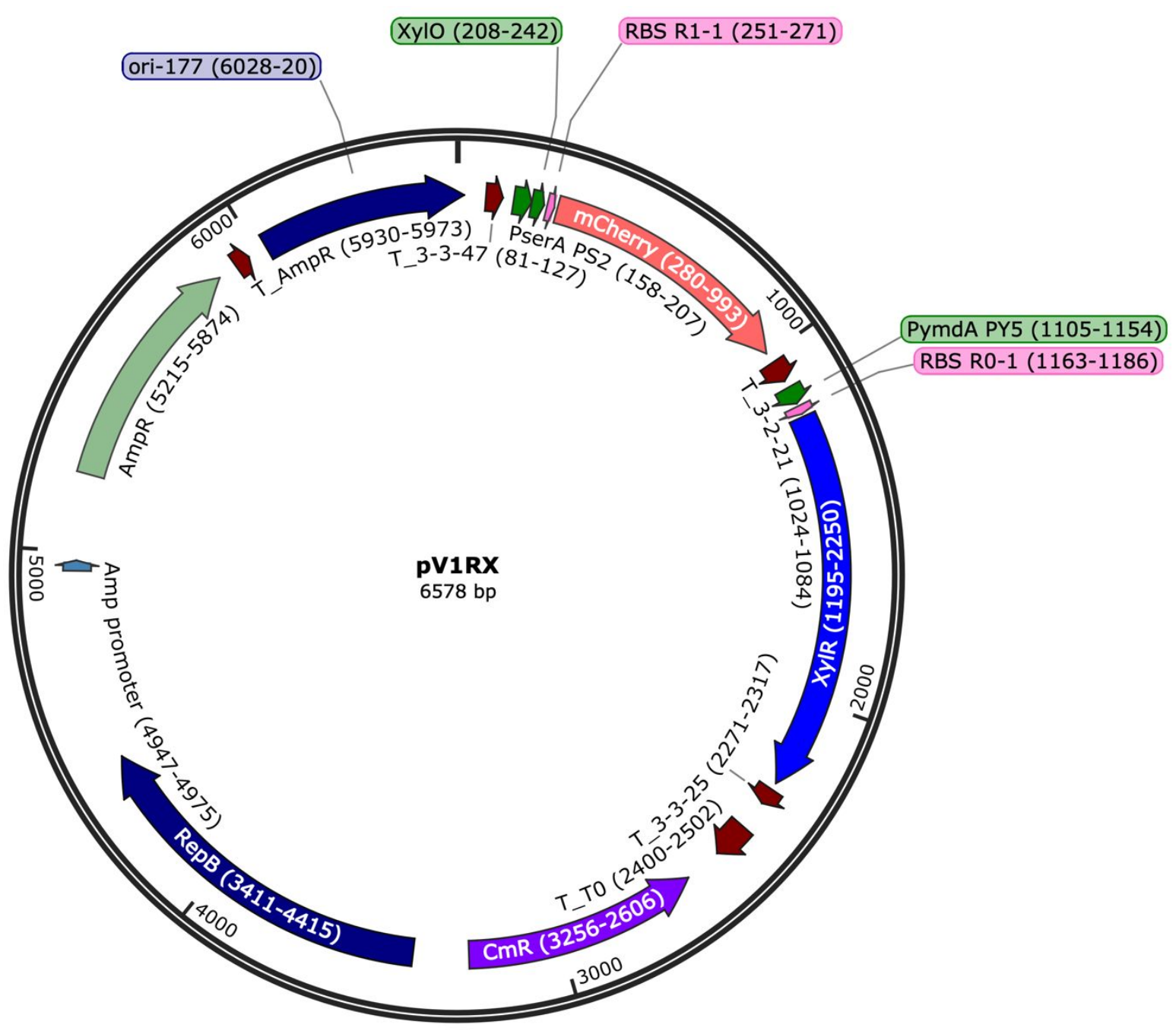


Supporting Figure 4. Map of plasmid pBb.

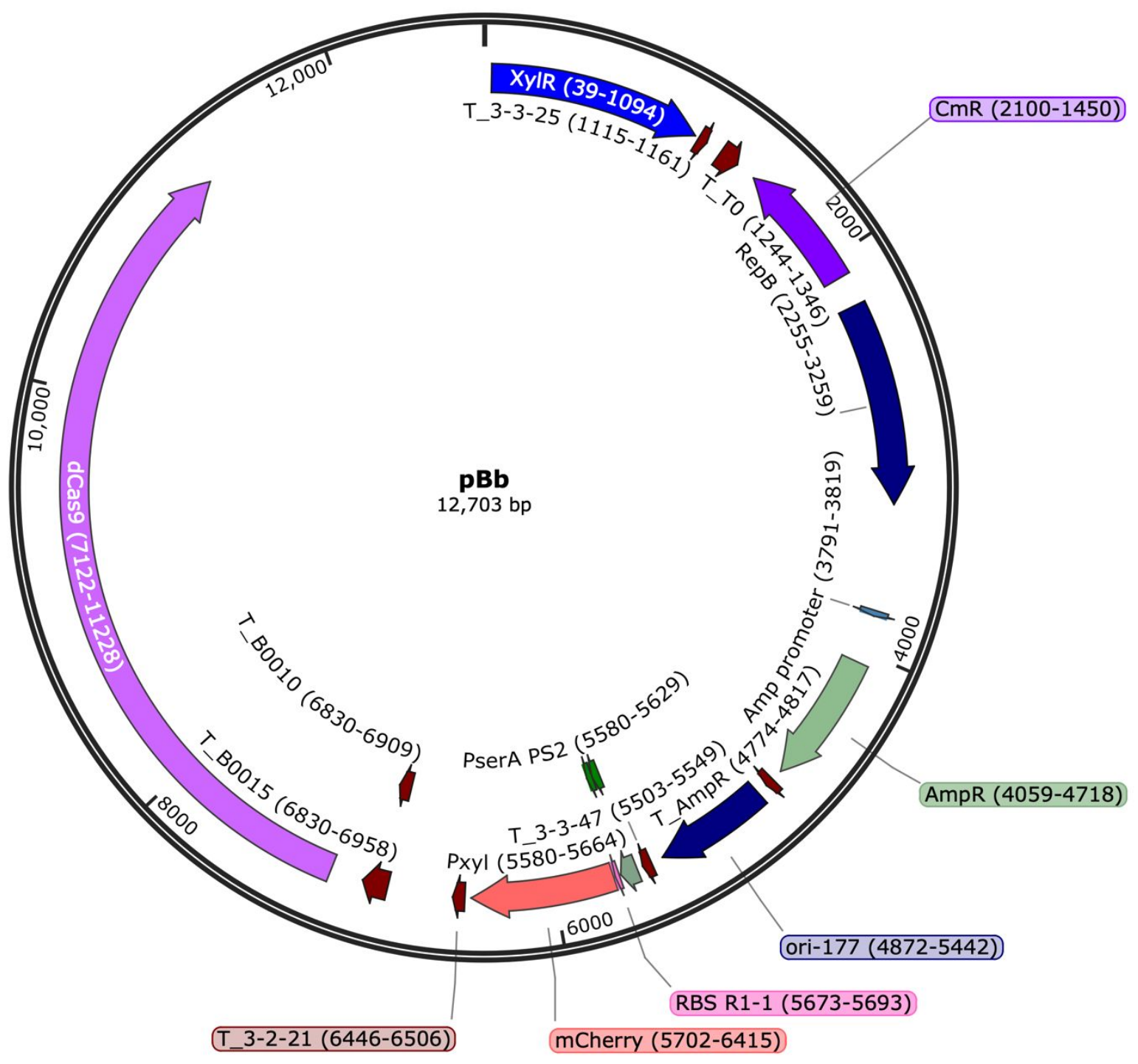


Supporting Figure 5. Map of plasmid pV1-PsspA.

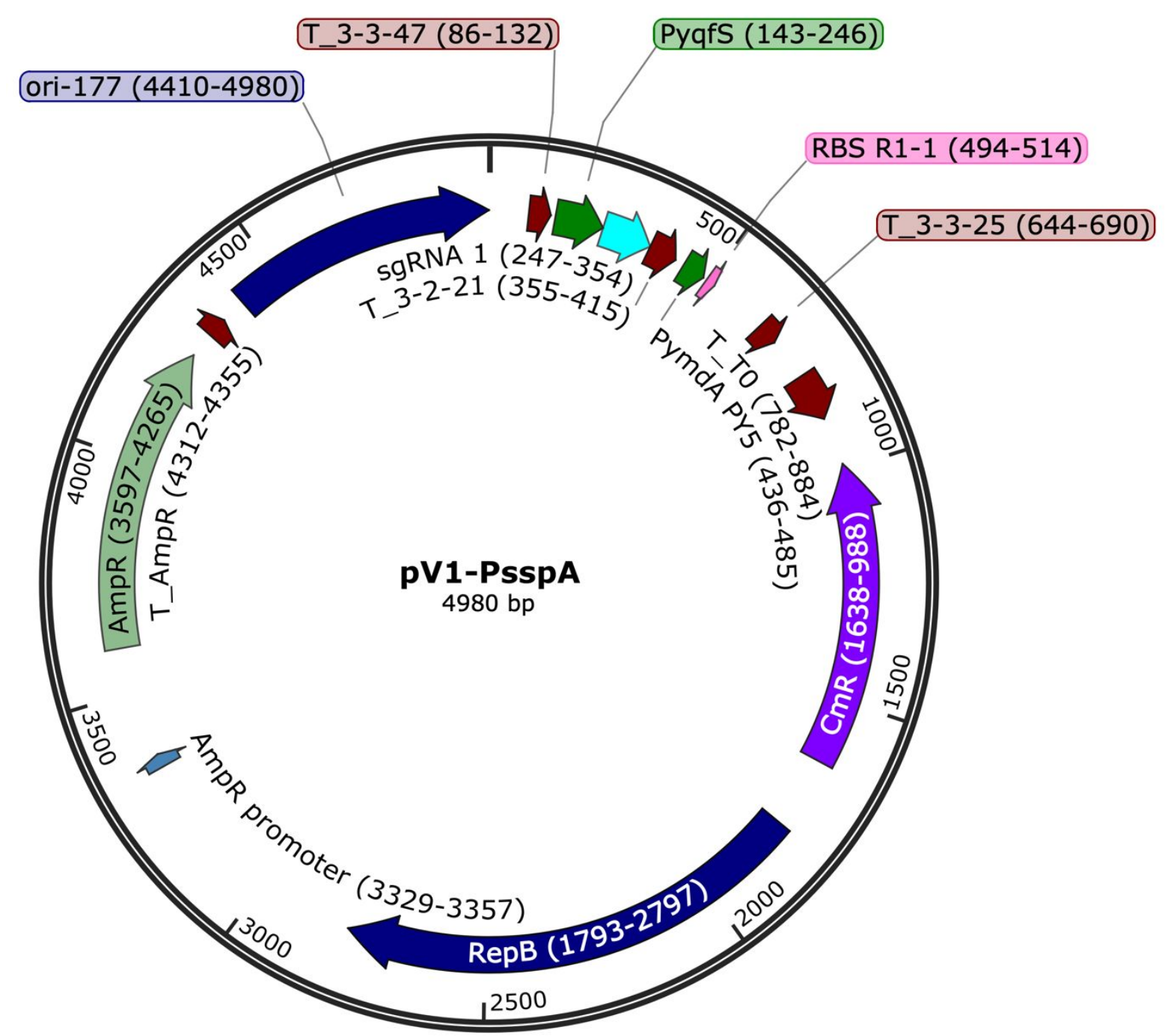


Supporting Figure 6. Map of plasmid pCas9g1-cons.

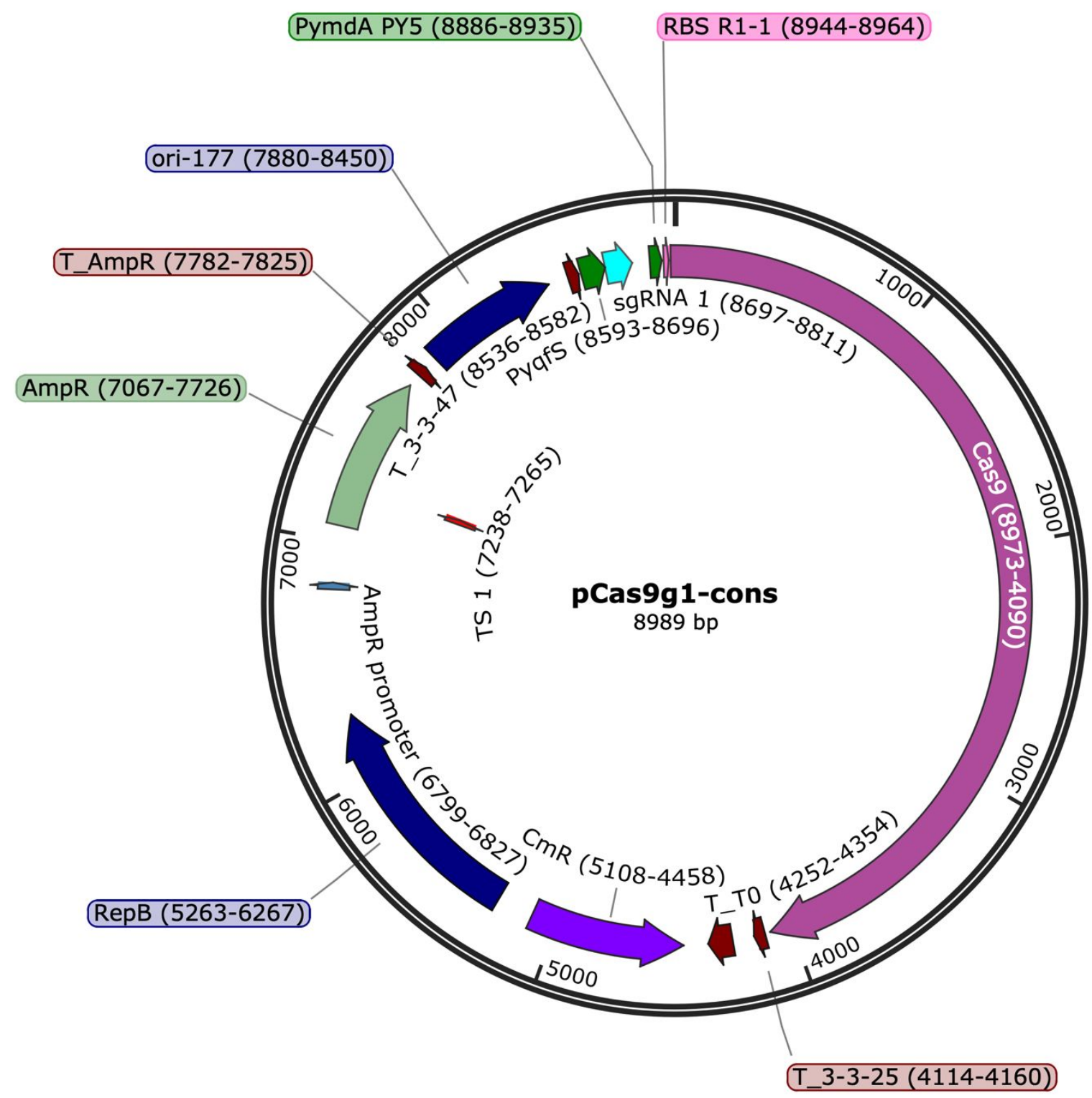


Supporting Figure 7. Map of plasmid pCas9g2-cons.

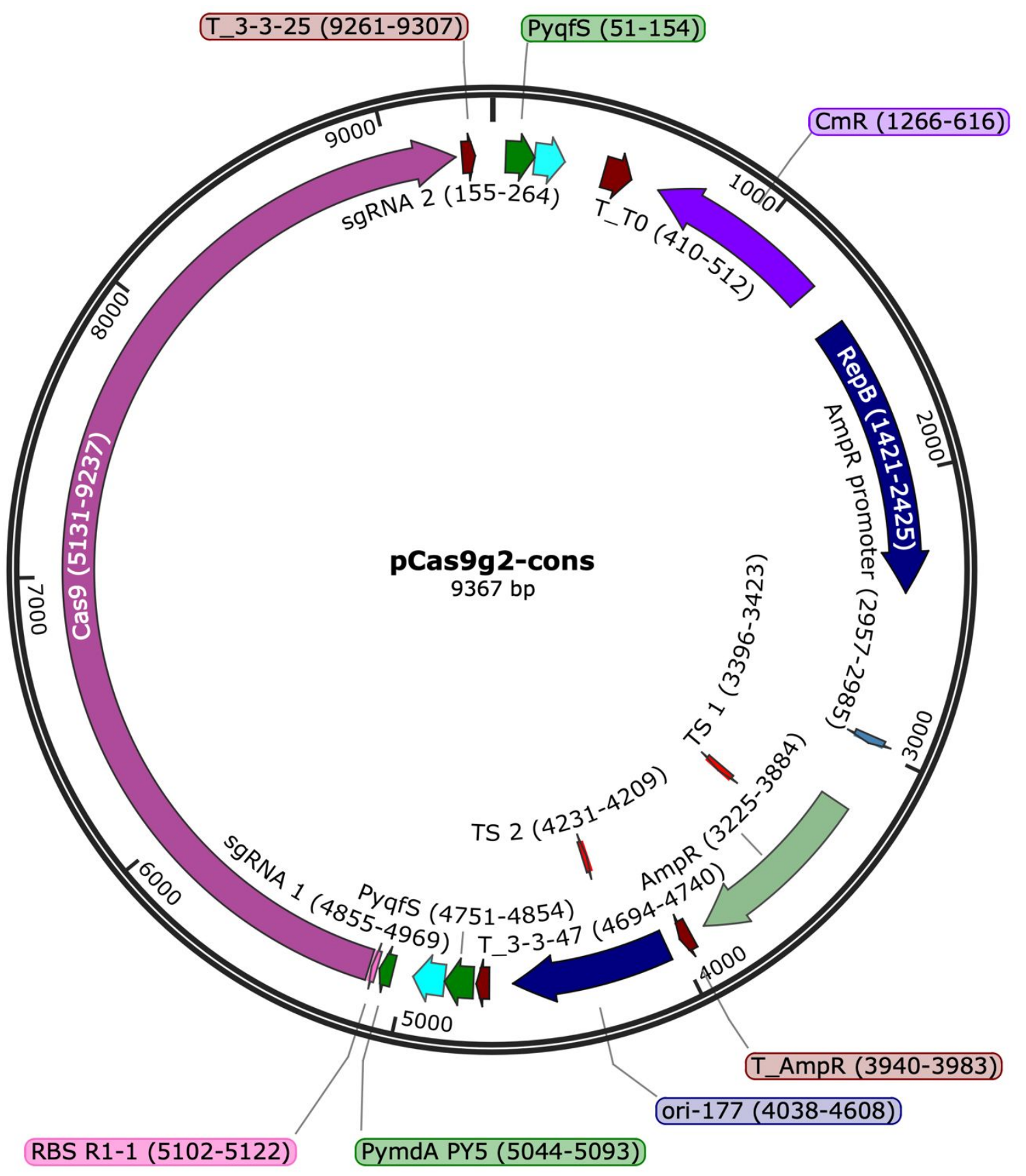


Supporting Figure 8. Map of plasmid pCas9g1-D15-cons.

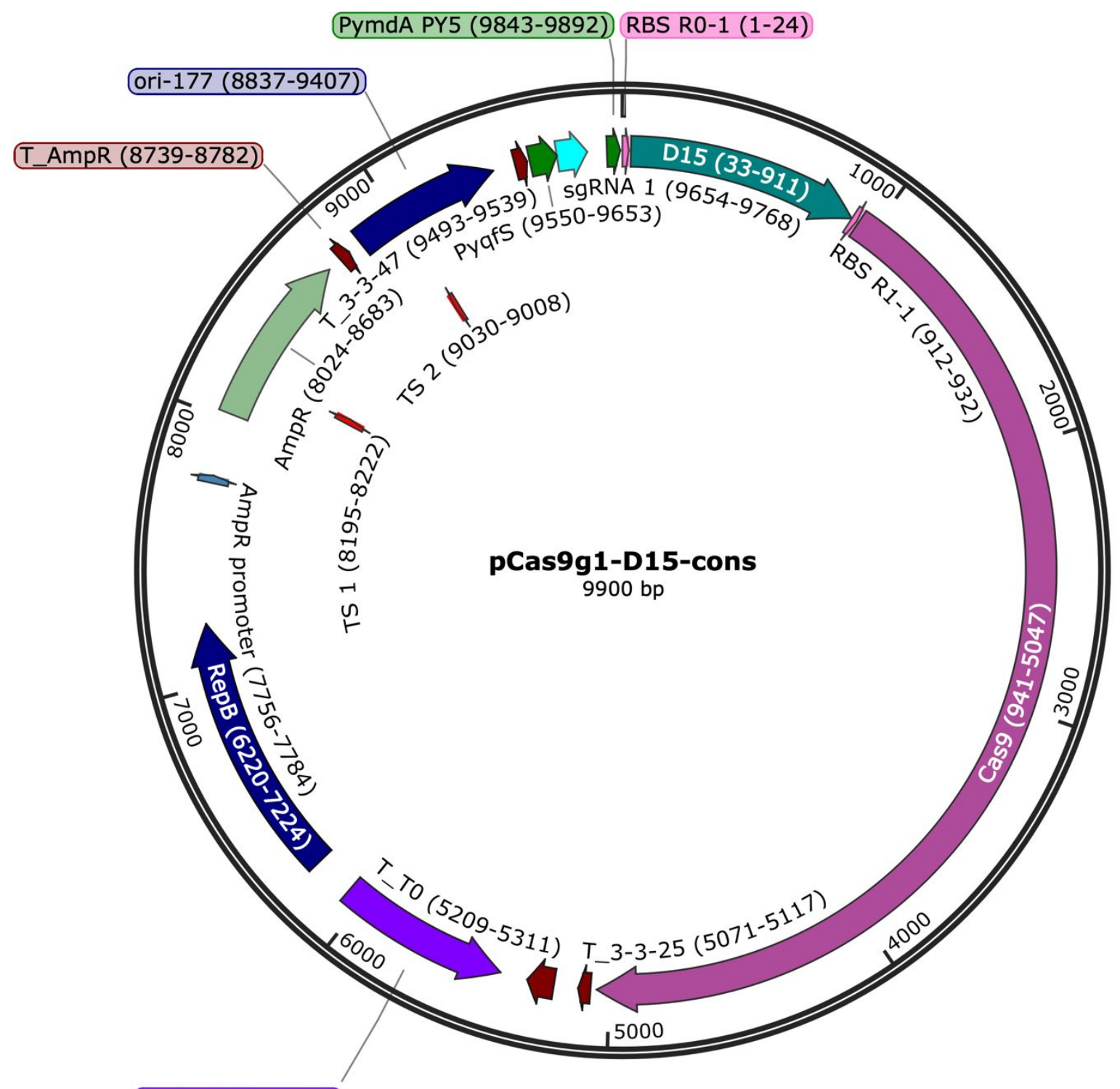

CmR (6065-5415) 
Supporting Figure 9. Map of plasmid pCas9g2-D15-cons.

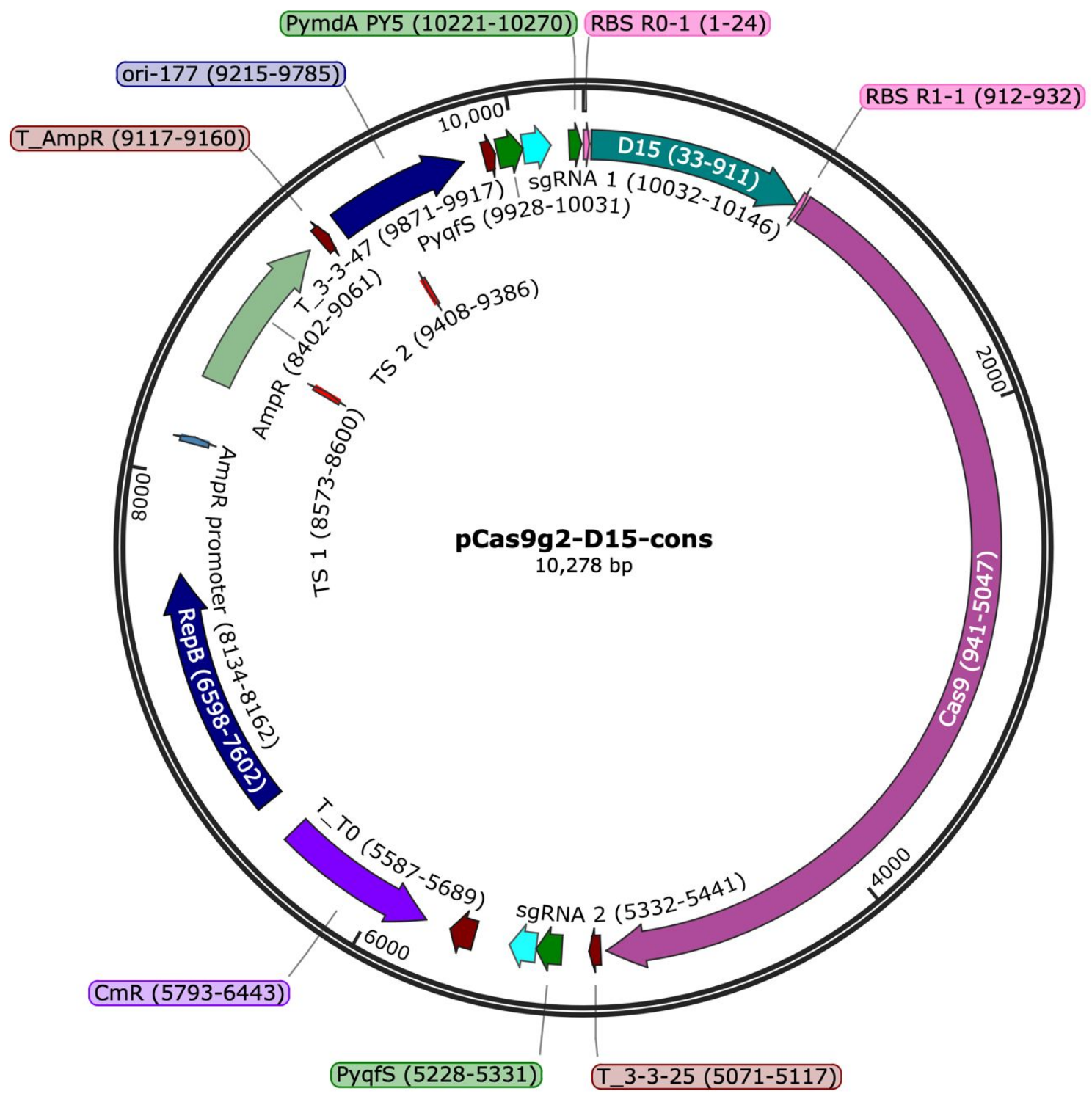


Supporting Figure 10. Map of plasmid pCas9g1.

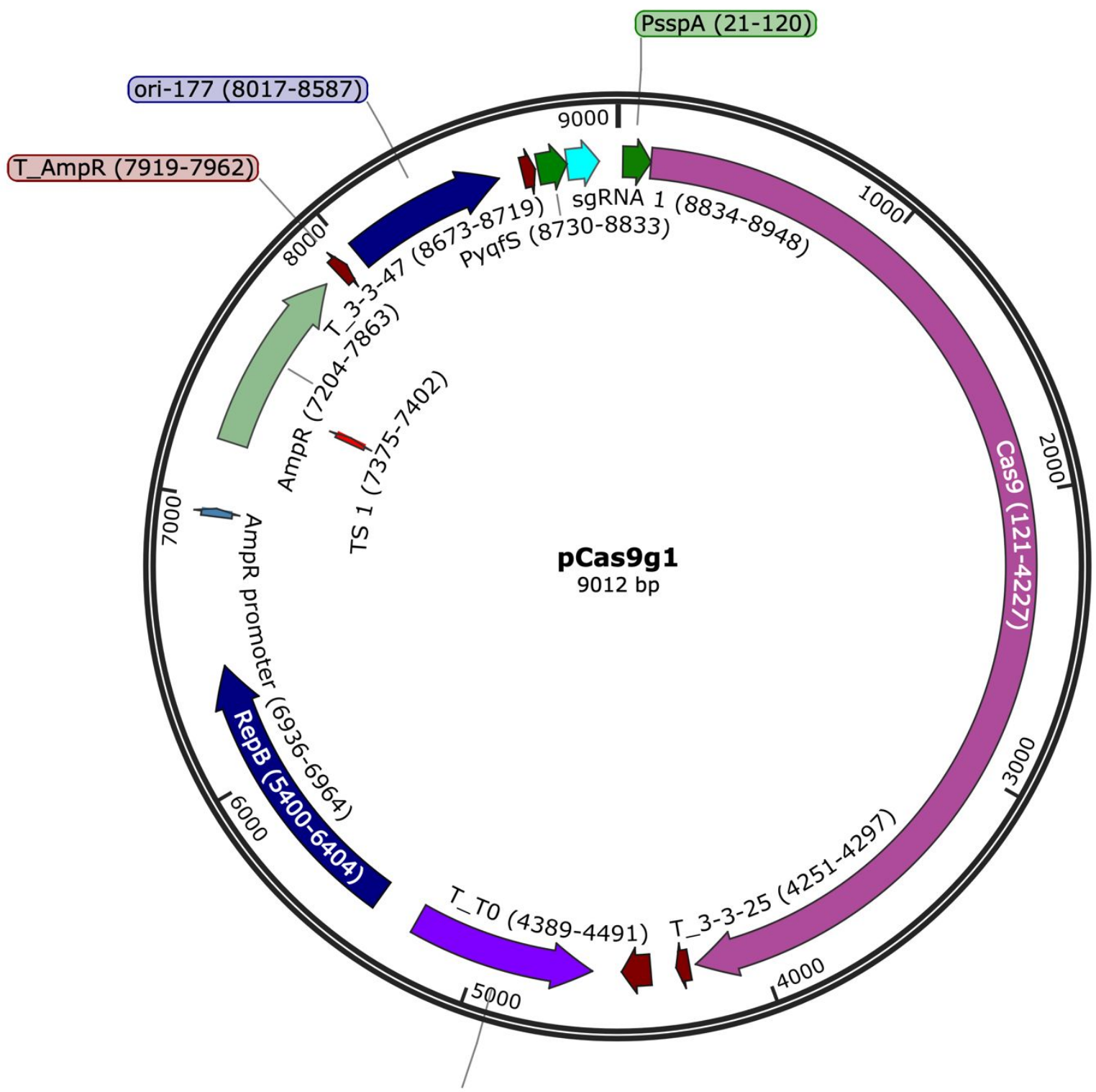

CmR (4595-5245) 
Supporting Figure 11. Map of plasmid pCas9g2.

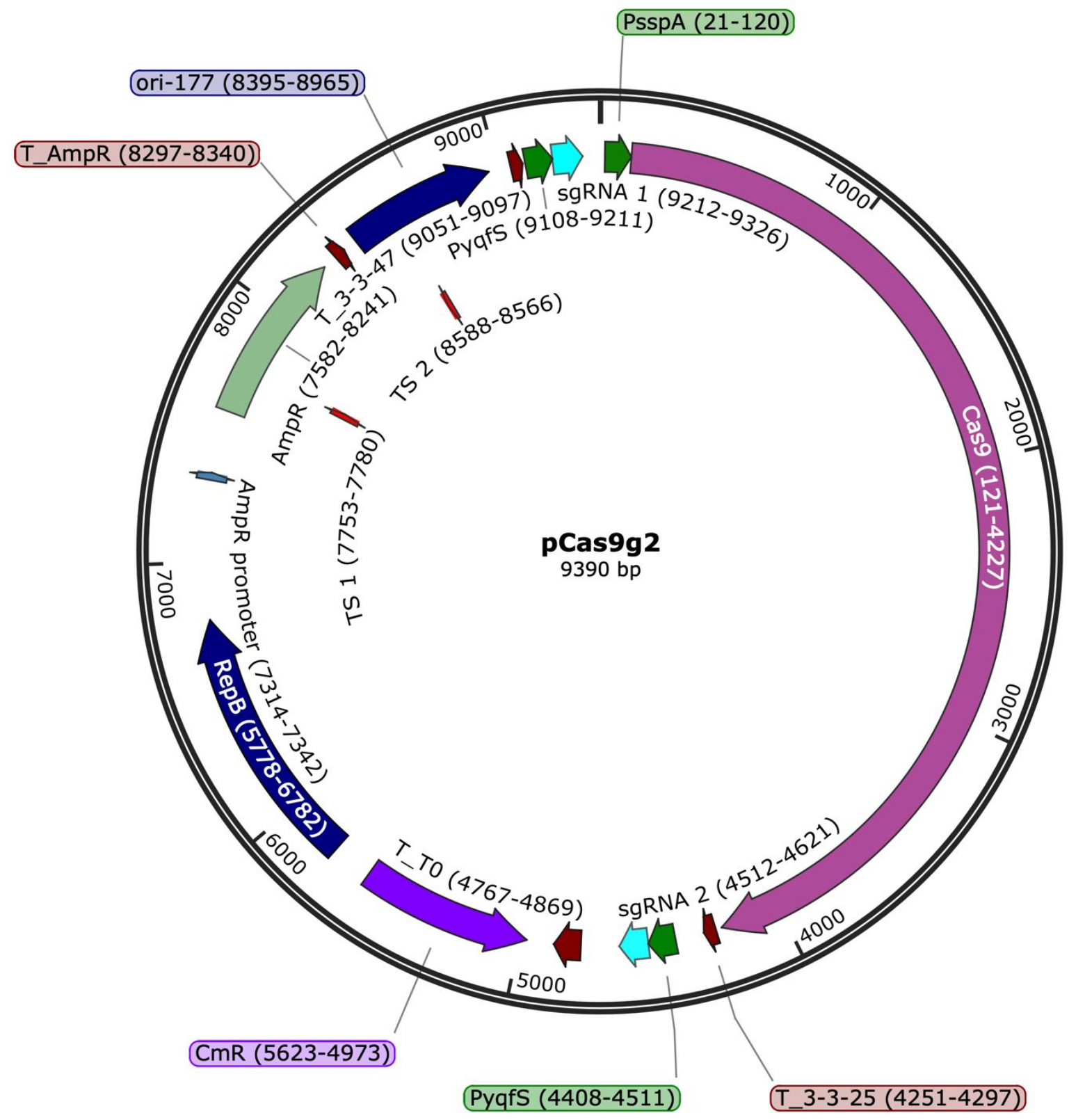


Supporting Figure 12. Map of plasmid pCas9g1-D15.

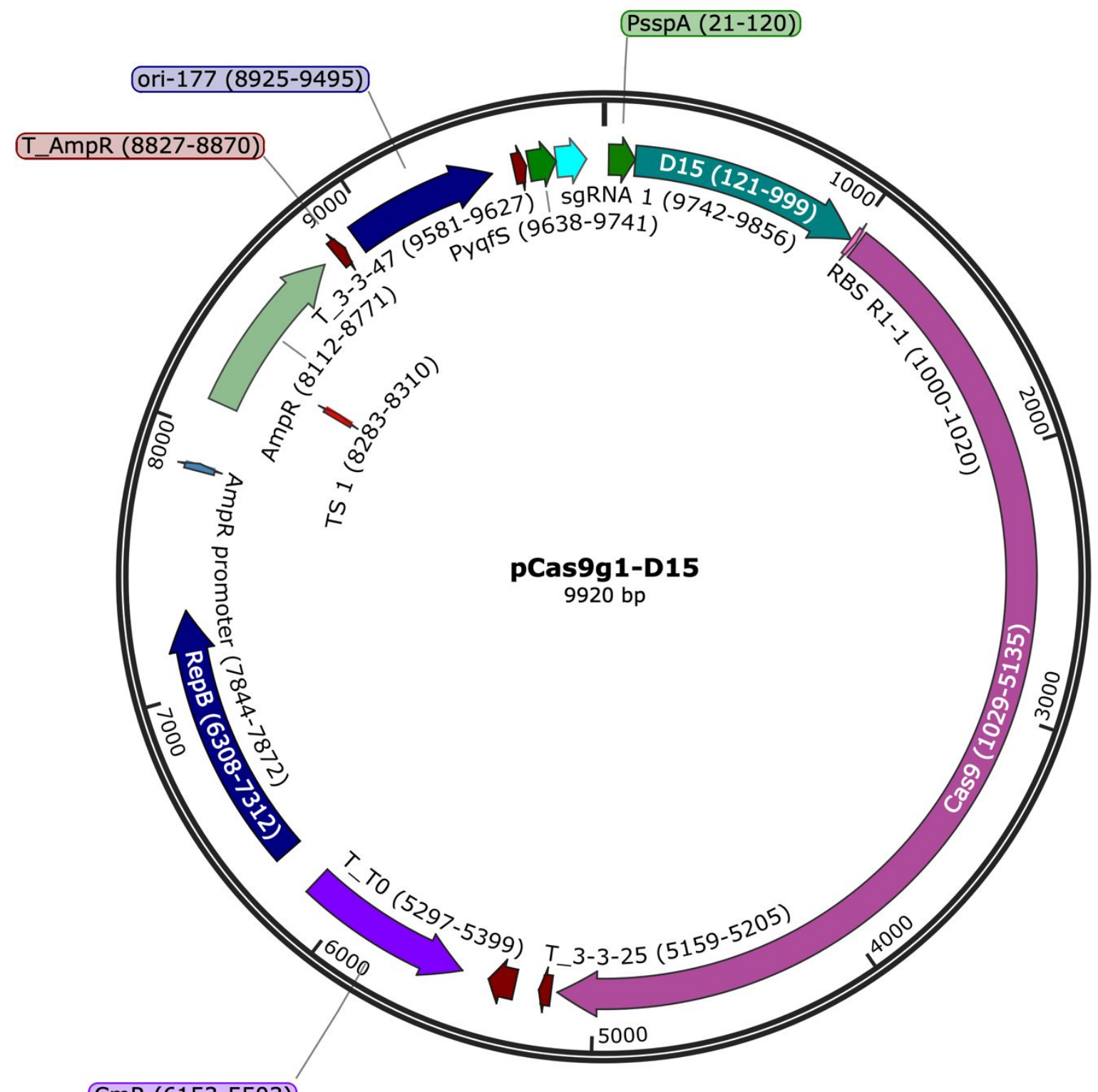

CmR (6153-5503) 
Supporting Figure 13. Map of plasmid pCas9g2-D15.

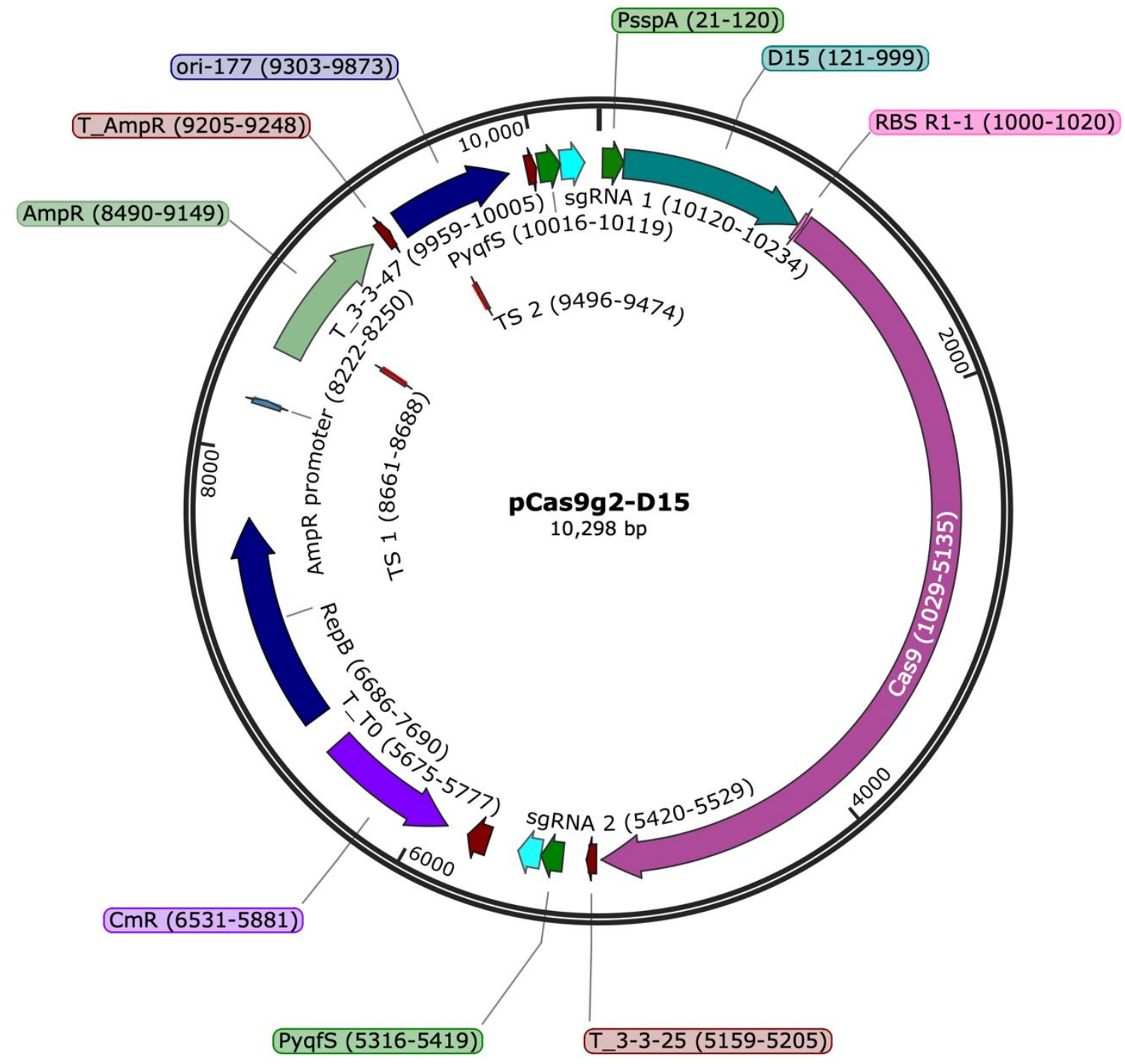


Supporting Figure 14. Map of plasmid pCas9g1-D15-mut.

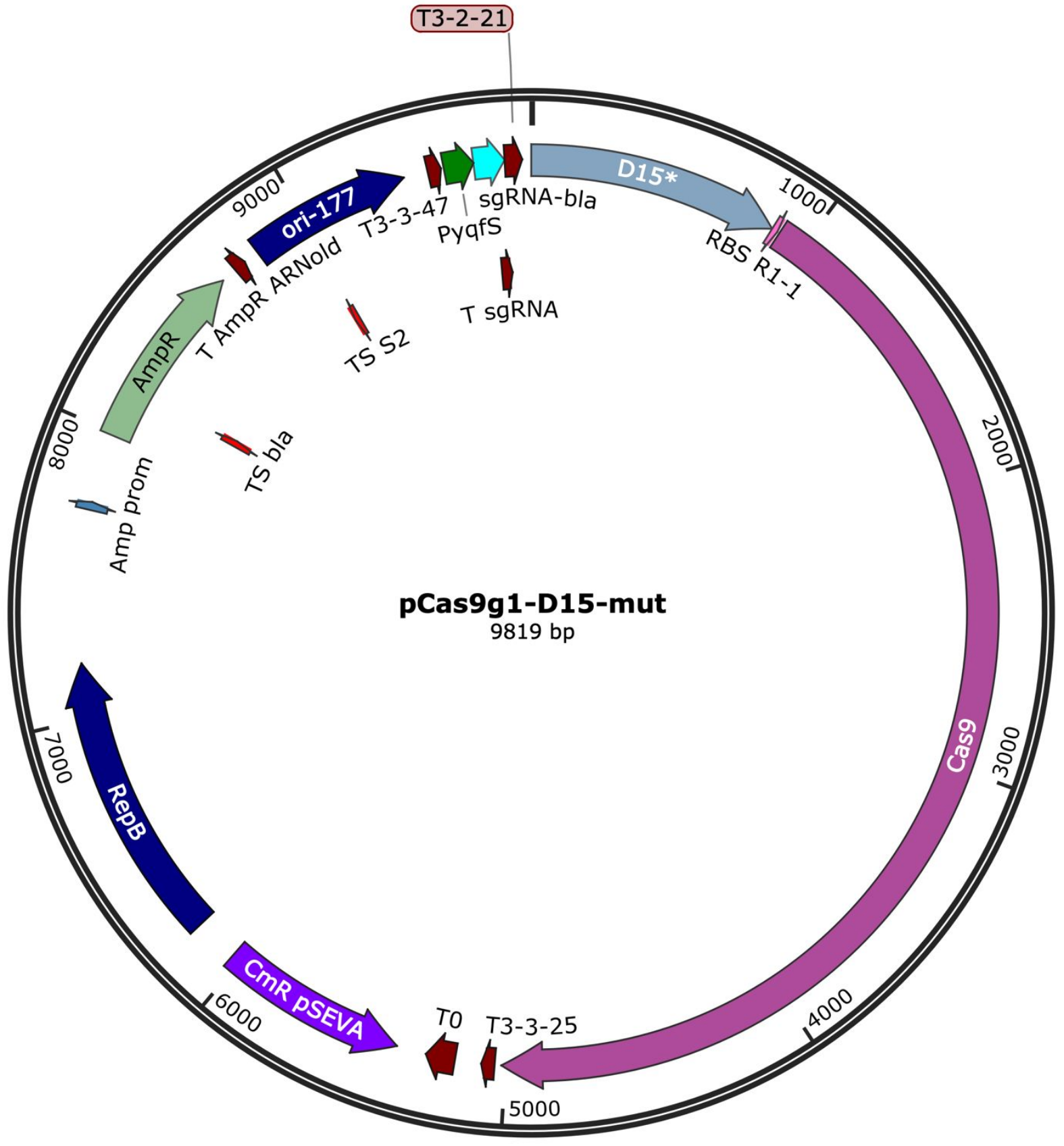


Supporting Figure 15. Map of plasmid pCas9g2-D15-mut.

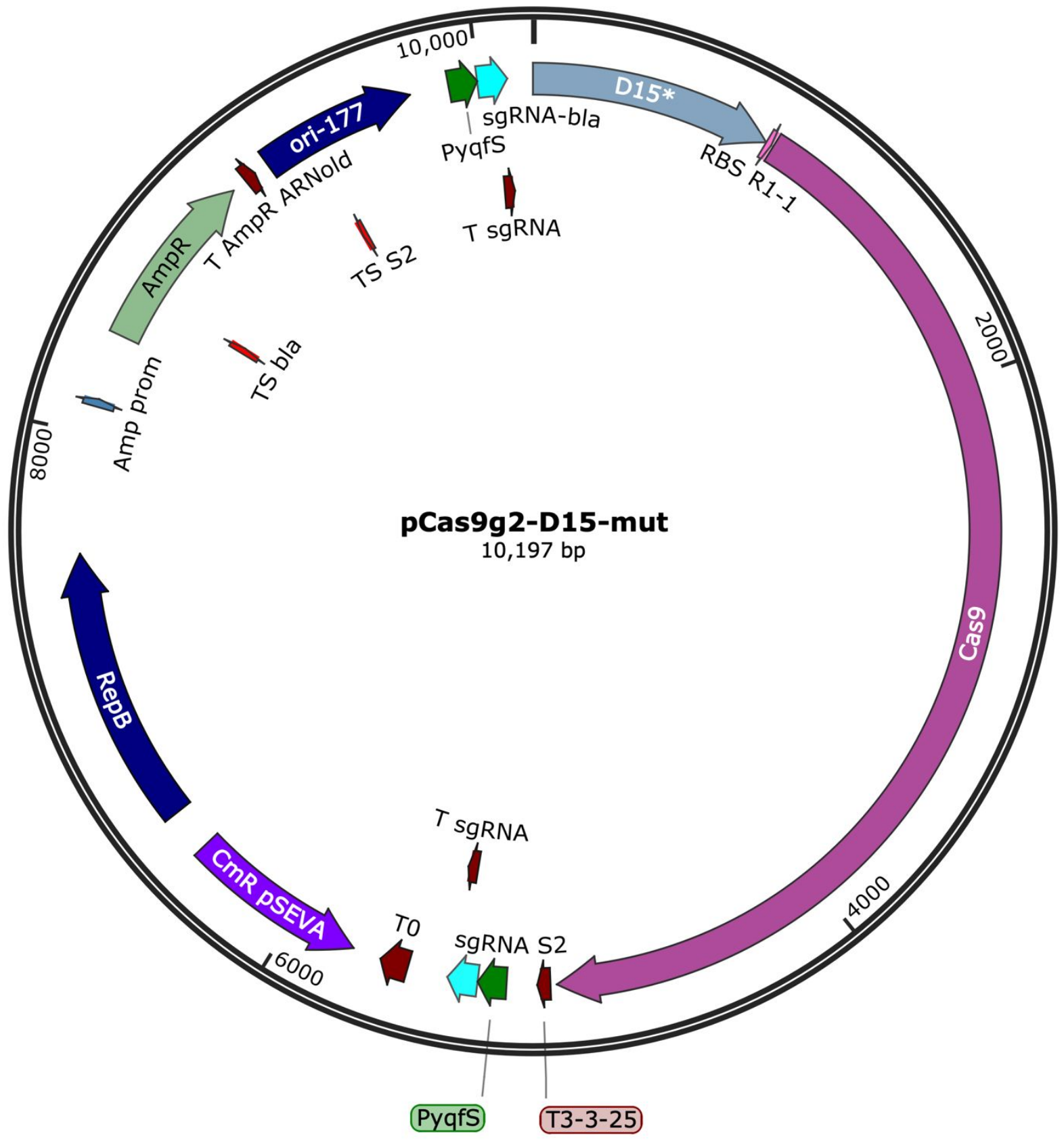


Supporting Figure 16. Map of plasmid pCas9g1-D15-GFP.

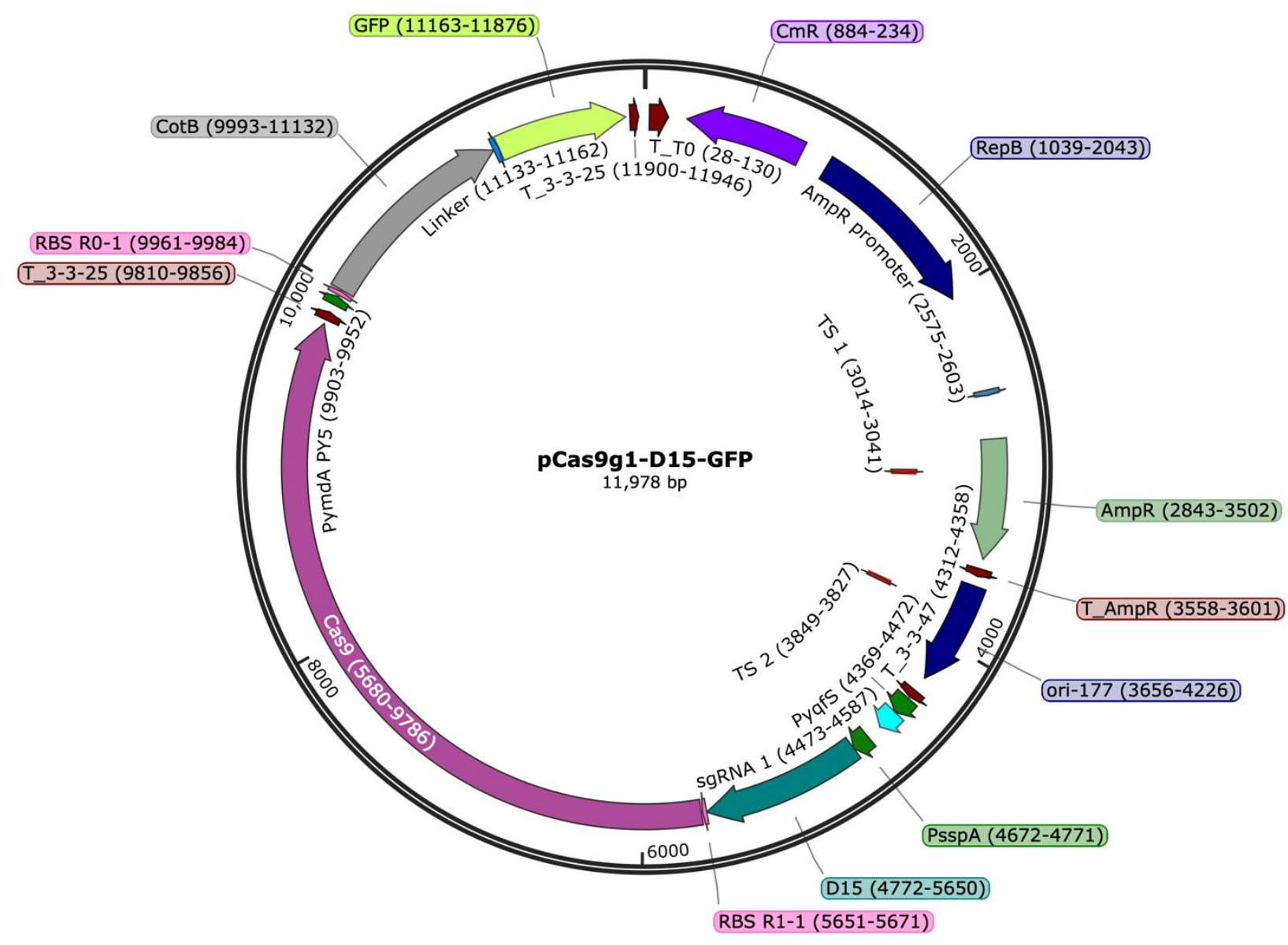


Supporting Figure 17. Map of plasmid pCas9g2-D15-GFP.

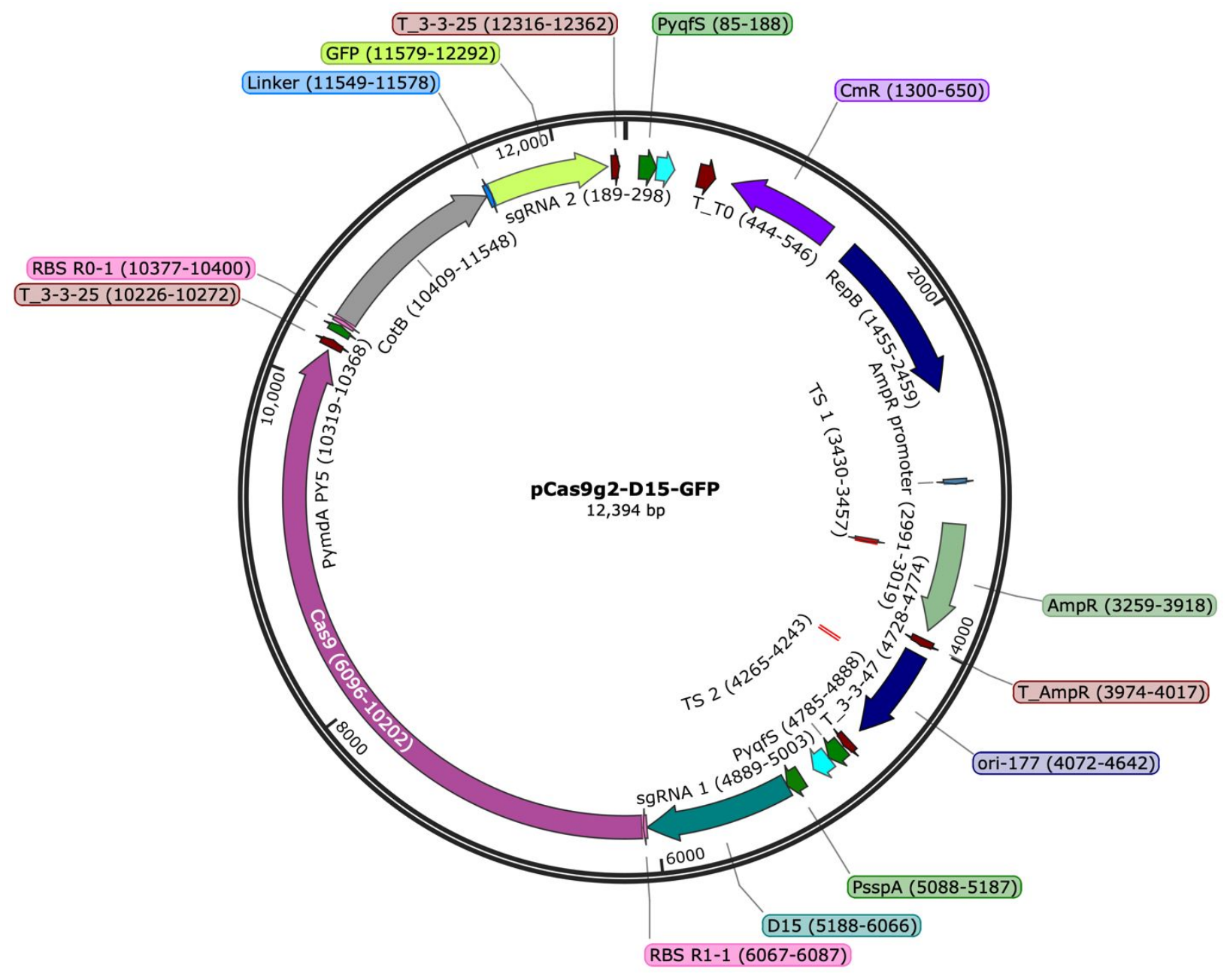


Supporting Figure 18. Map of plasmid pCas9g1-D15-KinA.

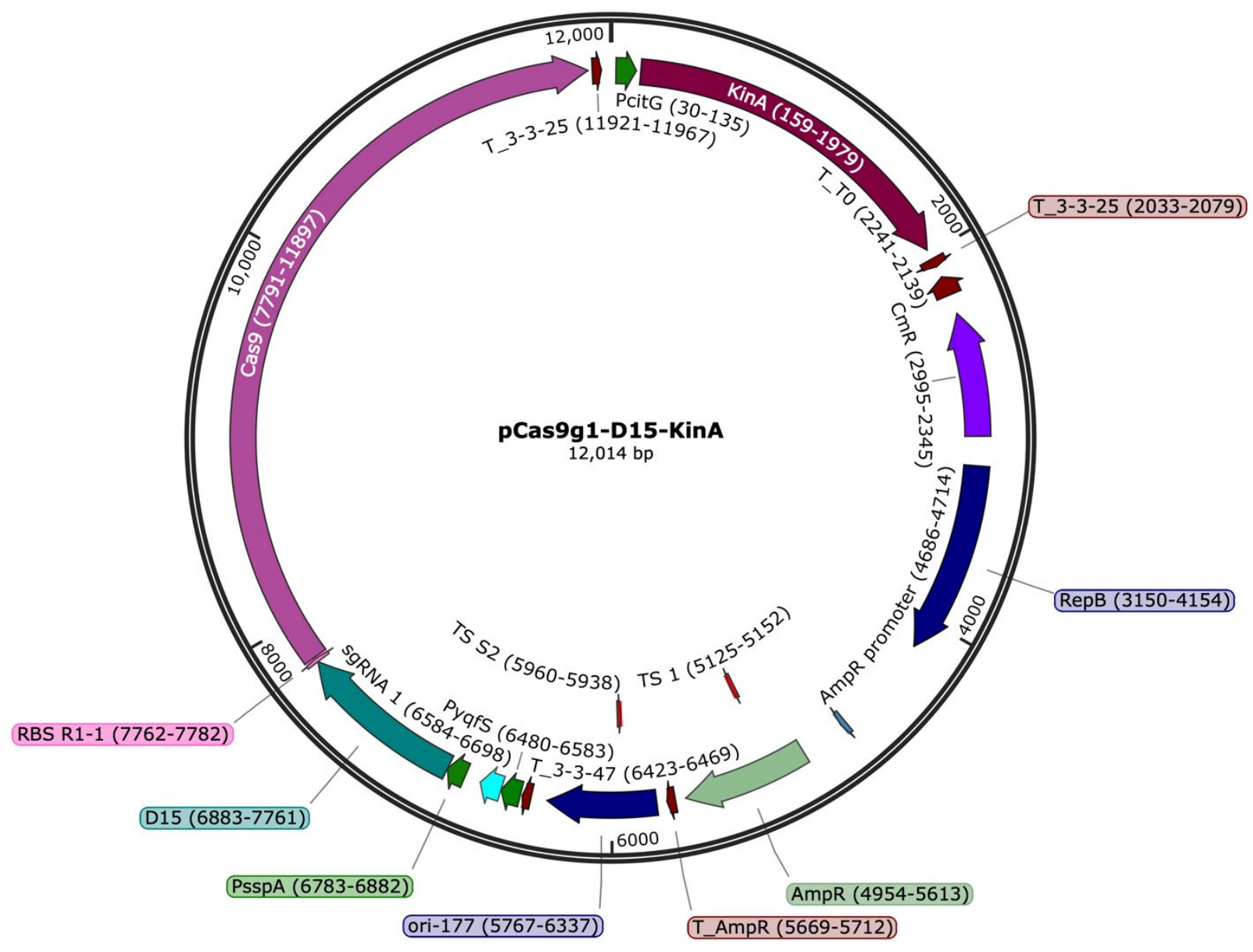


Supporting Figure 19. Map of plasmid pCas9g2-D15-KinA.

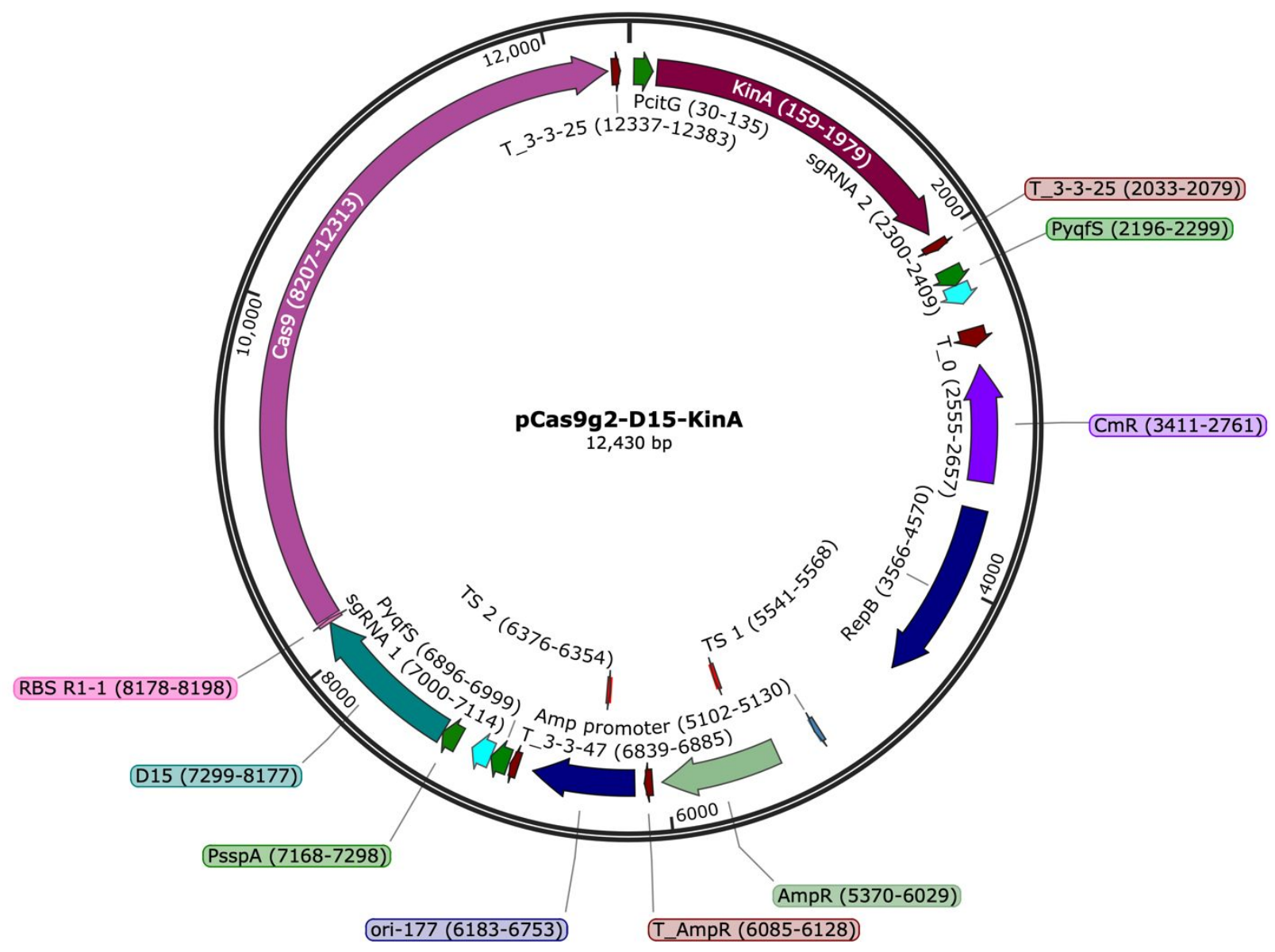


Supporting Figure 20. GFP-coated spores. A, C: GFP imaging with confocal microscopy. B, D: Optical microscopy. A-B: Strain pCas9g1-D15-GFP. C-D: Strain pCas9g2-D15-GFP.
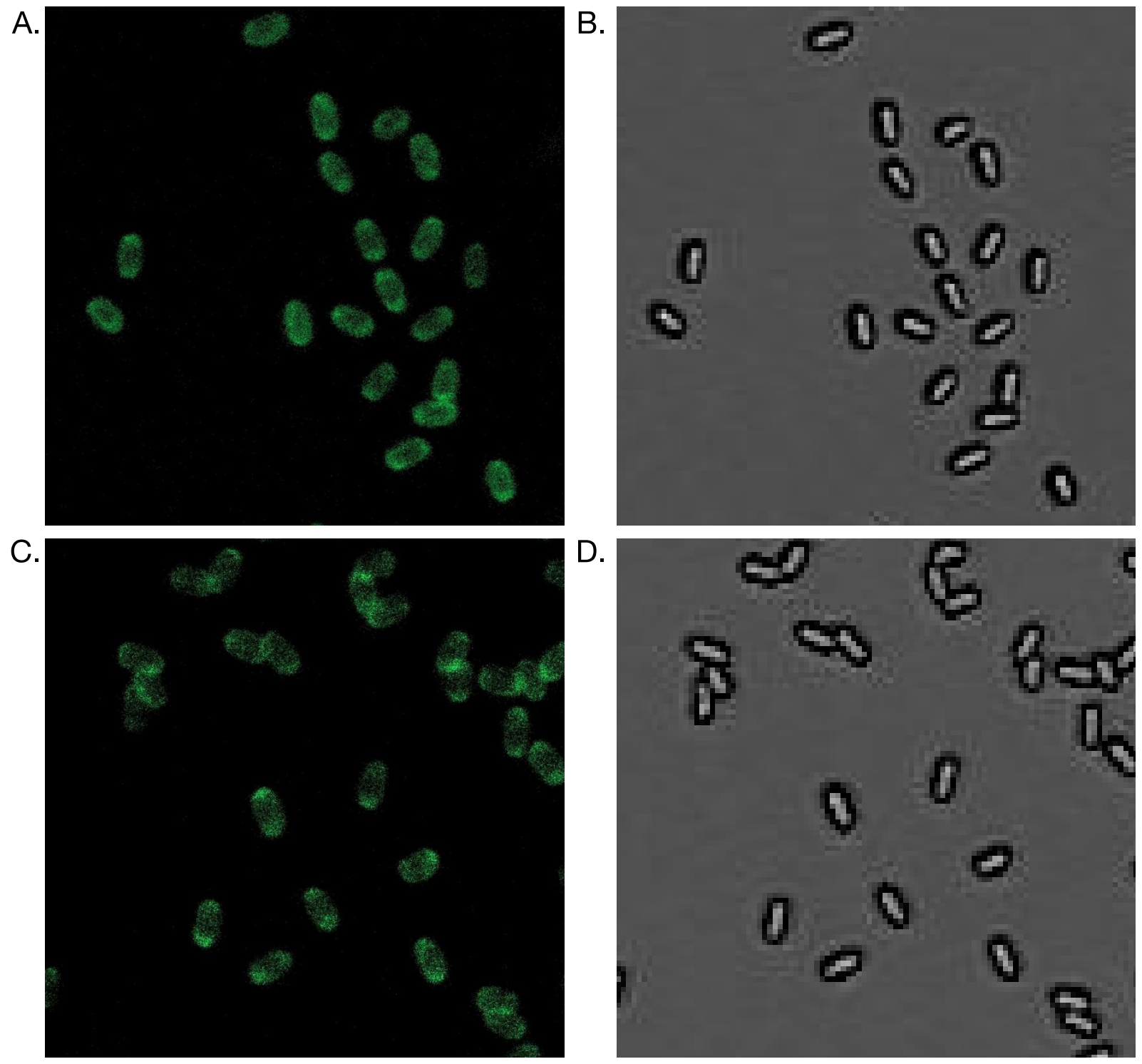
Supporting Figure 21. Germination of spore strains corresponding to mutated plasmids that were retained. Growth in LB-agar with or without antibiotic. $\mathrm{Cm}=$ chloramphenicol.
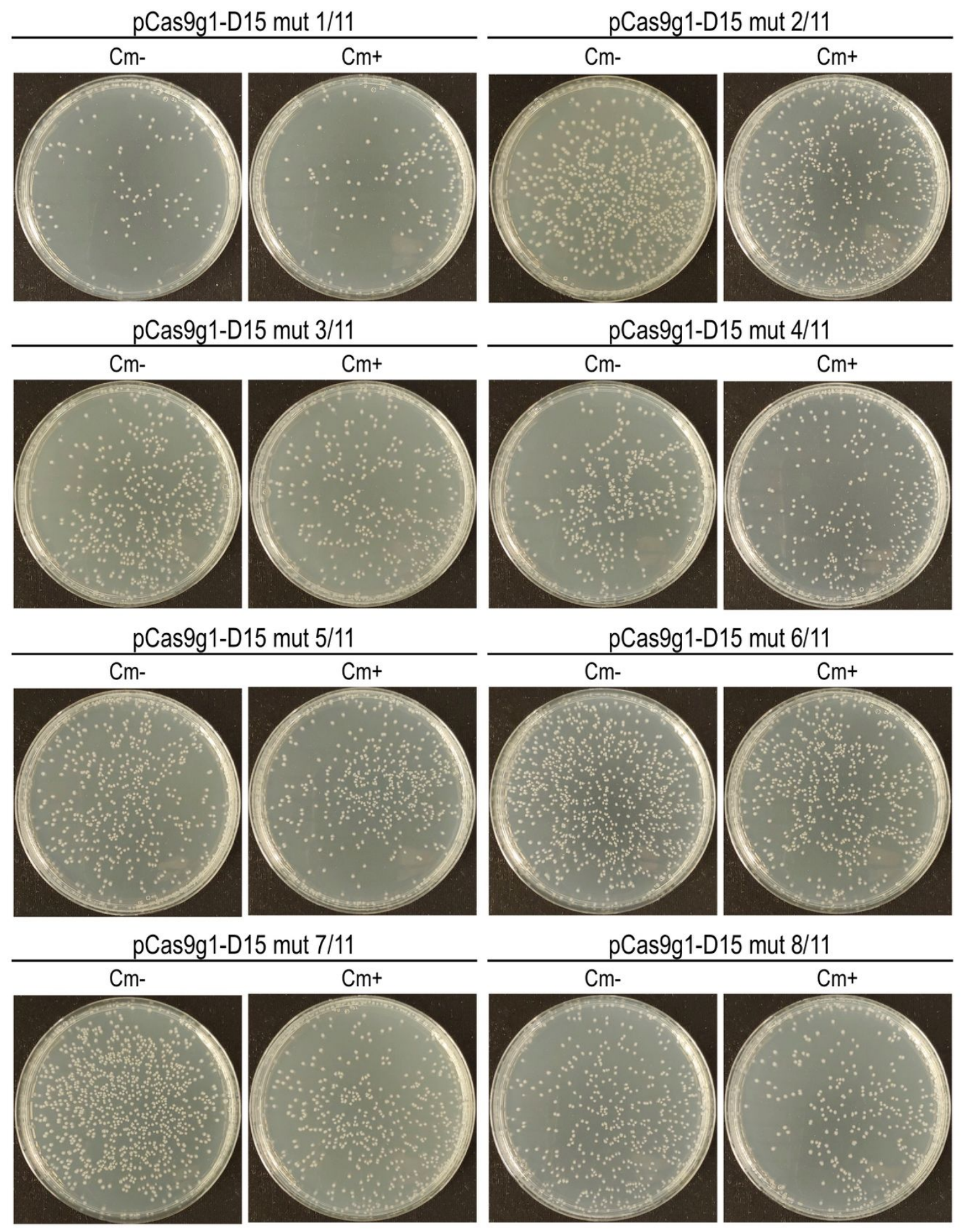
... continuation of Supporting Figure 21
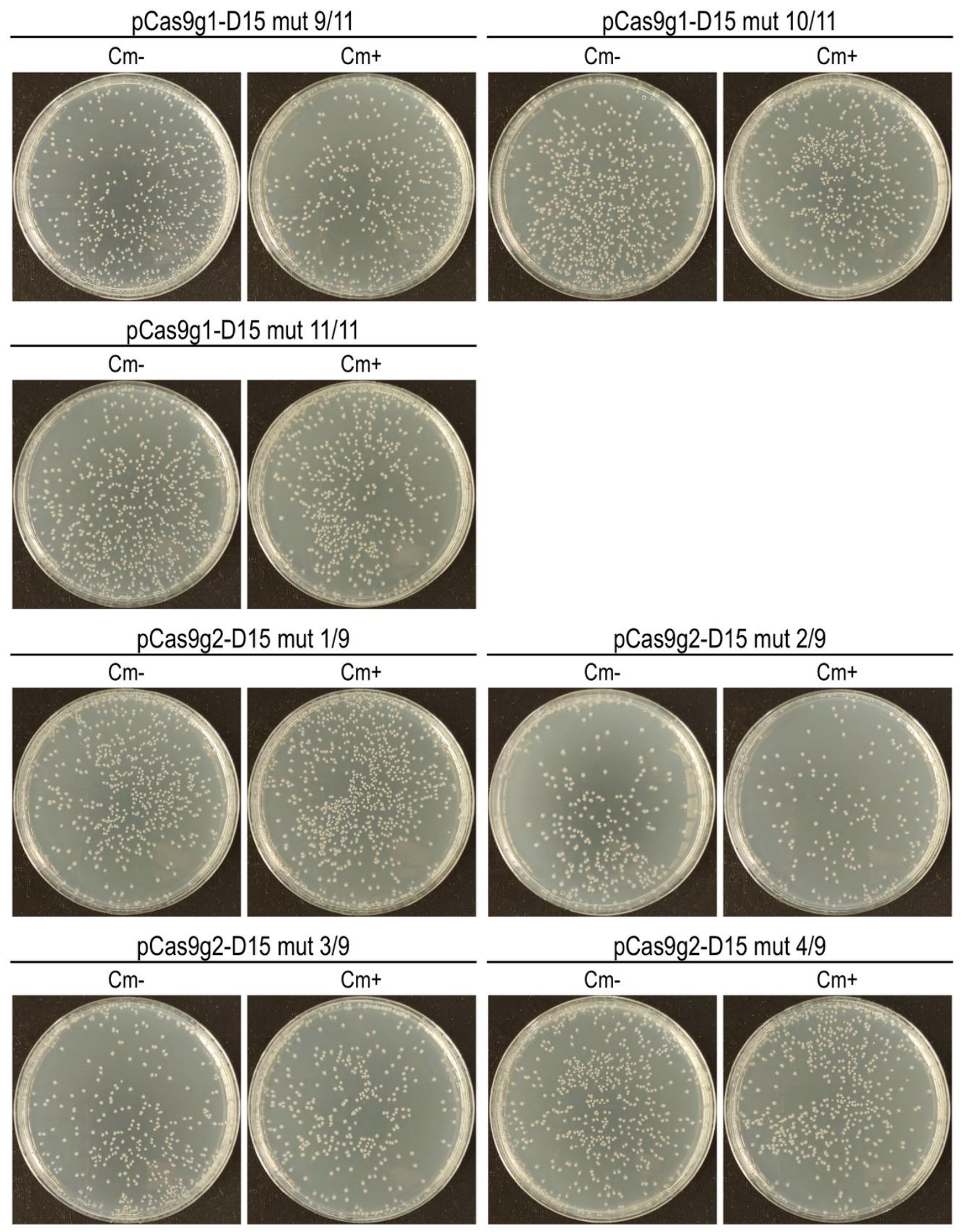
... continuation of Supporting Figure 21 

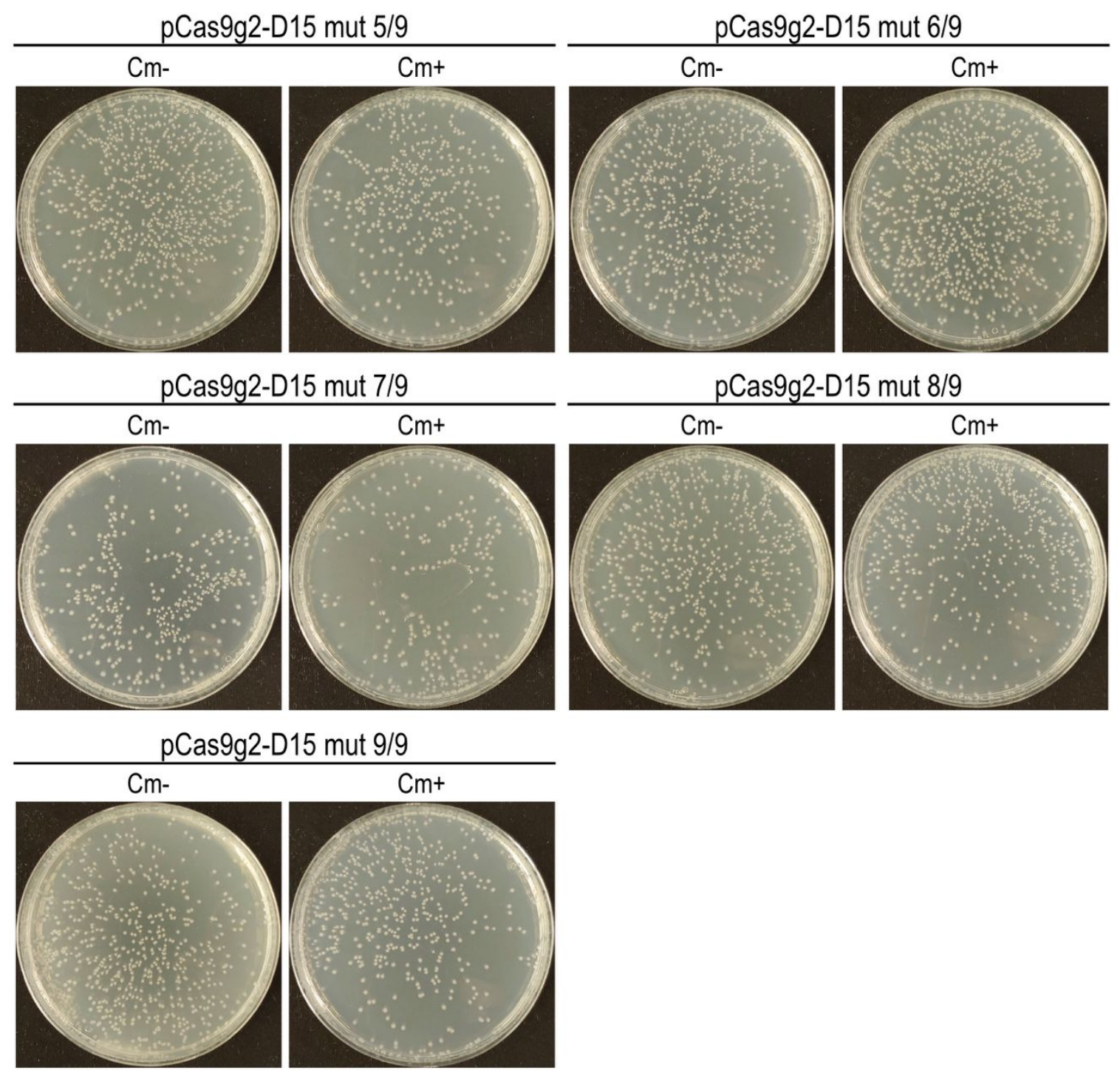
ttgtccttttccgctgcataaacaatatggcccgtttgttGAACTACTCTTTAATAAAATAATTTTTTCGTTCCCAATTCCACATTGCAATAATAG AAAATCCATCTTCATCGGCTTTTTCGTCATCATCTGTATGAATCAAATCGCCTTCTTCTGTGTCATCAAGGTTTAATTTTTTATGTA TTTCTTTTTAACAAACCACCATAGGAGATTAACCTTTTACGGTGTAAACCTTCCTCCAAATCAGACAAACGTTTCAAATTCTTTTTCT TCATCATCGGTCATAAAATCCGTATCCTTTACAGGATATTTTTGCAGTTTCGTCAATTGCCGATTGTATATCCGATTTATATTTATT TTTCGGTCGAATCATTTGAACTTTTACATTTGGATCATAGTCTAATTTCATTGCCTTTTTCCAAAATTGAATCCATTGTTTTTGATT CACGTAGTTTTCTGTATTCTTAAAATAAGTTGGTTCCACACATACCAATACATGCATGTGCTGATTATAAGAATTATCTTTATTAT TTATTGTCACTTCCGTTGCACGCATAAAACCAACAAGATTTTTATTAATTTTTTTATATTGCATCATTCGGCGAAATCCTTGAGCC ATATCTGACAAACTCTTATTTAATTCTTCGCCATCATAAACATTTTTAACTGTTAATGTGAGAAACAACCAACGAACTGTTGGCTT TTGTTTAATAACTTCAGCAACAACCTTTTGTGACTGAATGCCATGTTTCATTGCTCTCCTCCAGTTGCACATTGGACAAAGCCTG GATTTACAAAACCACACTCGATACAACTTTCTTTCGCCTGTTTCACGATTTTGTTTATACTCTAATATTTCAGCACAATCTTTTACT CTTTCAGCCTTTTTAAATTCAAGAATATGCAGAAGTTCAAAGTAATCAACATTAGCGATTTTCTTTTCTCTCCATGGTCTCACTTT TCCACTTTTTGTCTTGTCCACTAAAACCCTTGATTTTTCATCTGAATAAATGCTACTATTAGGACACATAATATTAAAAGAAACCC CCATCTATTTAGTTATTTGTTTGGTCACTTATAACTTTAACAGATGGGGTTTTTCTGTGCAACCAATTTTAAGGGTTTTCCAATAC TTTAAAACACATACATACCAACACTTCAACGCACCTTTCAGCAACTAAAATAAAAATGACGTTATTTCTATATGTATCAAGATAA GAAAGAACAAGTTCAAAACCATCAAAAAAAGACACCTTTTCAGGTGCTTTTTTTTATTTTATAAACTCATTCCCTGATCTCGACTT CGTTCTTTTTTTTACCTCTCGGTTATGAGTTAGTTCAAATTCGTTCTTTTTAGGTTCTAAATCGTGTTTTTCTTGGAATTGTGCTGTT TTATCCTTTACCTTGTCTACAAACCCCTTAAAAACGTTTTTAAAGGCTTTTAAGCGTCTGTACGTTCCTTAAGGAATTATTCCTTA GTGCTTTCTAGGTTAATGTCATGATAATAATGGTTTCTTAGACGTCAGGTGGCACTTTTCGGGGAAATGTCCGCGGAACCCCTA TTTGTTTATTTTTCTAAATACATTCAAATATGTATCCGCTCATGAGACAATAACCCTGATAAATGCTTCAATAATATTGAAAAAG GAAGAGTATGAGTATTCAACATTTCCGTGTCGCCCTTATTCCCTTTTTTGGGGCATTTTGCCTTCCTGTTTTTGCTCACCCAGAAA CGCTGGTGAAAGTAAAAGATGCTGAAGATCAGTTGGGTGCACGAGTGGGTTACATCGAACTGGATCTCAACAGCGGTAAGAT CCTTGAGAGTTTTCGCCCCGAAGAACGTTTTCCAATGATGAGCACTTTTAAAGTTCTGCTATGTGGCGCGGTATTATCCCGTGTT GACGCCGGGCAAGAGCAACTCGGTCGCCGCATACACTATTCTCAGAATGACTTGGTTGAGTACTCACCAGTCACAGAAAAGCA TCTTACGGATGGCATGACAGTAAGAGAATTATGCAGTGCTGCCATAACCATGAGTGATAACACTGCGGCCAACTTACTTCTGA CAACGATCGGAGGACCGAAGGAGCTAACCGCTTTTTTGCACAACATGGGGGATCATGTAACTCGCCTTGATCGTTGGGAACCG GAGCTGAATGAAGCCATACCAAACGACGAGCGTGACACCACGATGCCTGCAGCAATGGCAACAACGTTGCGCAAACTATTAA CTGGCGAACTACTTACTCTAGCTTCCCGGCAACAATTAATAGACTGGATGGAGGCGGATAAAGTTGCAGGACCACTTCTGCGC TCGGCCCTTCCGGCTGGCTGGTTTATTGCTGATAAATCTGGAGCCGGTGAGCGTGGGTCTCGCGGTATCATTGCAGCACTGGG GCCAGATGGTAAGCCCTCCCGTATCGTAGTTATCTACACGACGGGGAGTCAGGCAACTATGGATGAACGAAATAGACAGATC GCTGAGATAGGTGCCTCACTGATTAAGCATTGGTAACTGTCAGACCAAGTTTACTCATATATACTTTAGATTGATTTAAAACTTC ATTTTTAATTTAAAAGGATCTAGGTGAAGATCCTTTTTGATAATCTCATGACCAAAATCCCTTAACGTGAGTTTTCGTTCCACTG AGCGTCAGACCCCTTAATAAGATGATCTTCTTGAGATCGTTTTGGTCTGCGCGTAATCTCTTGCTCTGAAAACGAAAAAACCGC CTTGCAGGGAGGTTTTTCGAAGGTTCTCTGAGCTACCAACTCTTTGAACCGAGGTAACTGGCTTGCAGGAGCGCAGTCACCAA AACTTGTCCTTTCAGTTTAGCCTTAACCGGCGCATGACTTCAAGACTAACTCCTCTAAATCAATTACCAGTGGCTGCTGCCAGTG GTGCTTTTGCATGTCTTTCCGGGTTGGACTCAAGACGATAGTTACCGGATAAGGCGCAGCGGTCGGACTGAACGGGGGGTTC GTGCATACAGTCCAGCTTGGAGCGAACTGCCTACCCGGAACTGAGTGTCAGGCGTGGAATGAGACAAACGCGGCCATAACAG CGGAATGACACCGGTAAACCGAAAGGCAGGAACAGGAGAGCGCACGAGGGAGCCGCCAGGGGGAAACGCCTGGTATCTTTA TAGTCCTGTCGGGTTTCGCCACCACTGATTTGAGCGTCAGATTTCGTGATGCTTGTCAGGGGGCGGAGCCTATGGAAAAACGC TTTGCCcaagcttctagagatctgcagtgactgggaaaaccctggcGACTAGTCTTGGACTCCTGTTGATAGATCCAGTAATGACCTCAGAAC TCCATCTGGATTTGTTCAGAACGCTCGGTTGCCGCCGGGCGTTTTTTATTGGTGAGAATCCAGGGGTCCCCAATAATTACGATT TAAATCCTTCAAACTTCCCAAAGGCGAGCCCTAGTGACATTAGAAAACCGACTGTAAAAAGTACAGTCGGCATTATCTCATATT ATAAAAGCCAGTCATTAGGCCTATCTGACAATTCCTGAATAGAGTTCATAAACAATCCTGCATGATAACCATCACAAACAGAAT GATGTACCTGTAAAGATAGCGGTAAATATATTGAATTACCTTTATTAATGAATTTTTCCTGCTGTAATAATGGGTAGAAGGTAAT TACTATTATTATTGATATTTAAGTTAAACCCAGTAAATGAAGTCCATGGAATAATAGAAAGAGAAAAAGCATTTTCAGGTATAG GTGTTTTGGGAAACAATTTCCCCGAACCATTATATTTCTCTACATCAGAAAGGTATAAATCATAAAACTCTTTGAAGTCATTCTTT ACAGGAGTCCAAATACCAGAGAATGTTTTAGATACACCATCAAAAATTGTATAAAGTGGCTCTAACTTATCCCAATAACCTAAC TCTCCGTCGCTATTGTAACCAGTTCTAAAAGCTGTATTTGAGTTTATCACCCTTGTCACTAAGAAAATAAATGCAGGGTAAAATT TATATCCTTCTTGTTTTATGTTTCGGTATAAAACACTAATATCAATTTCTGTGGTTATACTAAAAGTCGTTTGTTGGTTCAAATAA TGATTAAATATCTCTTTTCTCTTCCAATTGTCTAAATCAATTTTATTAAAGTTCATTTGATATGCCTCCTAAATTTTTATCTAAAGT GAATTTAGGAGGCTTACTTGTCTGCTTTCTTCATTAGAATCAATCCTTTTTTAAAAGTCAATCCCGTTTGTTGAAGACTT

$>p V 1 R X$ 
tatggaaaaacgctttgccccacacaggctcttgaacaagGGATAACAATTTGTCATAGCTGTATTCGTTACAAGGAGGATTTTCGAAAAAAC ACCCTAACGGGTGTTTTTTTATAGCTGGTCTCCCTTGCTTGTACTTTACAGTATGCCATACGAAACTGCGTCAATACACGTTGAC ACTCTTTTGTAGTTATGTTAAATTATCAGATCGCCATCATTAGTTTGTTTAAACAACAAACTAATACTAGAGGCTCTTAAAGGGG GTTTTAGATACCAGAGATGGTGAGCAAGGGCGAGGAGGATAACATGGCCATCATCAAGGAGTTCATGCGCTTCAAGGTGCAC ATGGAGGGCTCCGTGAACGGCCACGAGTTCGAGATCGAGGGCGAGGGCGAGGGCCGCCCCTACGAGGGCACCCAGACCGCC AAGCTGAAGGTGACCAAGGGTGGCCCCCTGCCCTTCGCCTGGGACATCCTGTCCCCTCAGTTCATGTACGGCTCCAAGGCCTA CGTGAAGCACCCCGCCGACATCCCCGACTACTTGAAGCTGTCCTTCCCCGAGGGCTTCAAGTGGGAGCGCGTGATGAACTTCG AGGACGGCGGCGTGGTGACCGTGACCCAGGACTCCTCCCTGCAGGACGGCGAGTTCATCTACAAGGTGAAGCTGCGCGGCAC CAACTTCCCCTCCGACGGCCCCGTAATGCAGAAGAAGACCATGGGCTGGGAGGCCTCCTCCGAGCGGATGTACCCCGAGGAC GGCGCCCTGAAGGGCGAGATCAAGCAGAGGCTGAAGCTGAAGGACGGCGGCCACTACGACGCTGAGGTCAAGACCACCTAC AAGGCCAAGAAGCCCGTGCAGCTGCCCGGCGCCTACAACGTCAACATCAAGTTGGACATCACCTCCCACAACGAGGACTACAC CATCGTGGAACAGTACGAACGCGCCGAGGGCCGCCACTCCACCGGCGGCATGGACGAGCTGTACAAGTAATAGCTGCCACCT TTCTGAACTTTGGTAATGCGTCTCGGTACCAAATTCCAGAAAAGAGGCCTCCCGAAAGGGGGGCCTTTTTTCGTTTTGGTCCCT CAATGATCTCCTTATCCGGTTAAGATGGCAAGCTTGACAAGTATTTCTTCAGAATTTACAATGAAGTTTACTAGAGGATTAACTA ATAAGGAGGGCAAACTACTAGAGATGACGGGCCTGAACAAGAGCACCGTAAgcagccaagtgaatacactgatgaaggagtcaatggtgtt CGAGATCGGCCAGGGCCAGAGCTCAGGTGGCCGCCGCCCGGTAATGTTAGTCTTCAACAAGAAAGCGGGCTATAGTGTGGGC ATCGACGTCGGCGTAGACTACATCAACGGAATCCTTACGGATTTAGAGGGCACGATTGTGTTGGACCAGTATAGACACCTTGA GAGCAACAGCCCGGAGATCACTAAGGATATCCTTATCGACATGATCCACCATTTCATCACACAGATGCCTCAGAGCCCTTATGG ATTCATCGGCATCGGCATCTGTGTACCGGGTTTAATCGACAAGGACCAGAAGATCGTGTTTACGCCTAATAGCAATTGGCGTG ACATCGATCTGAAGAGCAGCATCCAGGAGAAATATAACGTAAGCGTGTTCATCGAGAACGAAGCGAACGCAGGAGCGTACG GCGAGAAGTTGTTCGGTGCAGCGAAGAACCATGACAATATCATCTATGTGTCAATTTCAACGGGCATCGGAATTGGCGTGATC ATTAATAACCACCTGTACCGTGGCGTGTCAGGATTTAGCGGTGAGATGGGCCACATGACGATCGATTTCAACGGACCGAAGTG TTCATGTGGCAATAGAGGCTGTTGGGAGCTTTACGCGAGCGAGAAAGCGCTGCTGAAGAGCTTGCAAACGAAGGAGAAGAA ACTCAGCTACCAGGACATCATCAATCTTGCACACCTTAACGACATTGGCACACTGAACGCGCTGCAGAACTTCGGCTTCTACCT GGGCATCGGATTGACAAACATCTTGAACACGTTCAATCCGCAGGCAGTGATCCTGCGCAACTCAATCATCGAGAGCCACCCGA TGGTGCTGAACAGCATGCGCTCAGAGGTGAGCAGCCGTGTGTACAGCCAGCTCGGAAACTCATACGAGCTGCTTCCGAGCAG TCTTGGCCAGAACGCTCCTGCGCTTGGCATGAGCAGCATCGTAATCGACCACTTCTTAGATATGATCACGATGTAATAGTCAAA TGTCAGACGAAAATGCCAATTATTGAAGCGGCTAACGCCGCTTTTTTTGTTTCTGGTCTCCCCATAGTTTAACAAGTGCtgtaaga agtcattcagcatagcttctagagatctgcagtGACTGGGAAAACCCTGGCGACTAGTCTTGGACTCCTGTTGATAGATCCAGTAATGACC TCAGAACTCCATCTGGATTTGTTCAGAACGCTCGGTTGCCGCCGGGCGTTTTTTATTGGTGAGAATCCAGGGGTCCCCAATAAT TACGATTTAAATCCTTCAAACTTCCCAAAGGCGAGCCCTAGTGACATTAGAAAACCGACTGTAAAAAGTACAGTCGGCATTATC TCATATTATAAAAGCCAGTCATTAGGCCTATCTGACAATTCCTGAATAGAGTTCATAAACAATCCTGCATGATAACCATCACAAA CAGAATGATGTACCTGTAAAGATAGCGGTAAATATATTGAATTACCTTTATTAATGAATTTTCCTGCTGTAATAATGGGTAGAA GGTAATTACTATTATTATTGATATTTAAGTTAAACCCAGTAAATGAAGTCCATGGAATAATAGAAAGAGAAAAAGCATTTTCAG GTATAGGTGTTTTGGGAAACAATTTCCCCGAACCATTATATTTCTCTACATCAGAAAGGTATAAATCATAAAACTCTTTGAAGTC ATTCTTTACAGGAGTCCAAATACCAGAGAATGTTTTAGATACACCATCAAAAATTGTATAAAGTGGCTCTAACTTATCCCAATAA CCTAACTCTCCGTCGCTATTGTAACCAGTTCTAAAAGCTGTATTTGAGTTTATCACCCTTGTCACTAAGAAAATAAATGCAGGGT AAAATTTATATCCTTCTTGTTTTATGTTTCGGTATAAAACACTAATATCAATTTCTGTGGTTATACTAAAAGTCGTTTGTTGGTTC AAATAATGATTAAATATCTCTTTTCTCTTCCAATTGTCTAAATCAATTTTATTAAAGTTCATTTGATATGCCTCCTAAATTTTTATCT AAAGTGAATTTAGGAGGCTTACTTGTCTGCTTTCTTCATTAGAATCAATCCTTTTTTTAAAAGTCAATCCCGTTTGTTGAAGACTTT TGTCCTTTTCCGCTGCATAAACAATATGGCCCGTTTGTTGAACTACTCTTTAATAAAATAATTTTTCCGTTCCCAATTCCACATTG CAATAATAGAAAATCCATCTTCATCGGCTTTTTCGTCATCATCTGTATGAATCAAATCGCCTTCTTCTGTGTCATCAAGGTTTAAT TTTTTATGTATTTCTTTTAACAAACCACCATAGGAGATTAACCTTTTACGGTGTAAACCTTCCTCCAAATCAGACAAACGTTTCAA ATTCTTTTCTTCATCATCGGTCATAAAATCCGTATCCTTTACAGGATATTTTGCAGTTTCGTCAATTGCCGATTGTATATCCGATTT ATATTTATTTTTCGGTCGAATCATTTGAACTTTTACATTTGGATCATAGTCTAATTTCATTGCCTTTTTTCCAAAATTGAATCCATTG TTTTTGATTCACGTAGTTTTCTGTATTCTTAAAATAAGTTGGTTCCACACATACCAATACATGCATGTGCTGATTATAAGAATTAT CTTTATTATTTATTGTCACTTCCGTTGCACGCATAAAACCAACAAGATTTTTATTAATTTTTTTATATTGCATCATTCGGCGAAATC CTTGAGCCATATCTGACAAACTCTTATTTAATTCTTCGCCATCATAAACATTTTTAACTGTTAATGTGAGAAACAACCAACGAAC TGTTGGCTTTTGTTTAATAACTTCAGCAACAACCTTTTGTGACTGAATGCCATGTTTCATTGCTCTCCTCCAGTTGCACATTGGAC AAAGCCTGGATTTACAAAACCACACTCGATACAACTTTCTTTTCGCCTGTTTCACGATTTTGTTTATACTCTAATATTTCAGCACAA TCTTTTACTCTTTCAGCCTTTTTTAAATTCAAGAATATGCAGAAGTTCAAAGTAATCAACATTAGCGATTTTCTTTTCTCTCCATGG TCTCACTTTTCCACTTTTTGTCTTGTCCACTAAAACCCTTGATTTTTCATCTGAATAAATGCTACTATTAGGACACATAATATTAAA AGAAACCCCCATCTATTTAGTTATTTGTTTGGTCACTTATAACTTTAACAGATGGGGTTTTTCTGTGCAACCAATTTTAAGGGTTT 
TCCAATACTTTAAAACACATACATACCAACACTTCAACGCACCTTTCAGCAACTAAAATAAAAATGACGTTATTTCTATATGTATC AAGATAAGAAAGAACAAGTTCAAAACCATCAAAAAAAGACACCTTTTCAGGTGCTTTTTTTATTTTATAAACTCATTCCCTGATC TCGACTTCGTTCTTTTTTTTACCTCTCGGTTATGAGTTAGTTCAAATTCGTTCTTTTTTAGGTTCTAAATCGTGTTTTTCTTGGAATTG TGCTGTTTTATCCTTTACCTTGTCTACAAACCCCTTAAAAACGTTTTTAAAGGCTTTTAAGCGTCTGTACGTTCCTTAAGGAATTA TTCCTTAGTGCTTTCTAGGTTAATGTCATGATAATAATGGTTTCTTAGACGTCAGGTGGCACTTTTCGGGGAAATGTCCGCGGA ACCCCTATTTGTTTATTTTTCTAAATACATTCAAATATGTATCCGCTCATGAGACAATAACCCTGATAAATGCTTCAATAATATTG AAAAAGGAAGAGTATGAGTATTCAACATTTCCGTGTCGCCCTTATTCCCTTTTTTGGGGCATTTTGCCTTCCTGTTTTTGCTCACC CAGAAACGCTGGTGAAAGTAAAAGATGCTGAAGATCAGTTGGGTGCACGAGTGGGTTACATCGAACTGGATCTCAACAGCGG TAAGATCCTTGAGAGTTTTTCGCCCCGAAGAACGTTTTCCAATGATGAGCACTTTTAAAGTTCTGCTATGTGGCGCGGTATTATC CCGTGTTGACGCCGGGCAAGAGCAACTCGGTCGCCGCATACACTATTCTCAGAATGACTTGGTTGAGTACTCACCAGTCACAG AAAAGCATCTTACGGATGGCATGACAGTAAGAGAATTATGCAGTGCTGCCATAACCATGAGTGATAACACTGCGGCCAACTTA CTTCTGACAACGATCGGAGGACCGAAGGAGCTAACCGCTTTTTTGCACAACATGGGGGATCATGTAACTCGCCTTGATCGTTG GGAACCGGAGCTGAATGAAGCCATACCAAACGACGAGCGTGACACCACGATGCCTGCAGCAATGGCAACAACGTTGCGCAAA CTATTAACTGGCGAACTACTTACTCTAGCTTCCCGGCAACAATTAATAGACTGGATGGAGGCGGATAAAGTTGCAGGACCACT TCTGCGCTCGGCCCTTCCGGCTGGCTGGTTTATTGCTGATAAATCTGGAGCCGGTGAGCGTGGGTCTCGCGGTATCATTGCAG CACTGGGGCCAGATGGTAAGCCCTCCCGTATCGTAGTTATCTACACGACGGGGAGTCAGGCAACTATGGATGAACGAAATAG ACAGATCGCTGAGATAGGTGCCTCACTGATTAAGCATTGGTAACTGTCAGACCAAGTTTACTCATATATACTTTAGATTGATTTA AAACTTCATTTTTAATTTAAAAGGATCTAGGTGAAGATCCTTTTTTGATAATCTCATGACCAAAATCCCTTAACGTGAGTTTTCGTT CCACTGAGCGTCAGACCCCTTAATAAGATGATCTTCTTGAGATCGTTTTGGTCTGCGCGTAATCTCTTGCTCTGAAAACGAAAA AACCGCCTTGCAGGGAGGTTTTTTCGAAGGTTCTCTGAGCTACCAACTCTTTGAACCGAGGTAACTGGCTTGCAGGAGCGCAGT CACCAAAACTTGTCCTTTCAGTTTAGCCTTAACCGGCGCATGACTTCAAGACTAACTCCTCTAAATCAATTACCAGTGGCTGCTG CCAGTGGTGCTTTTGCATGTCTTTCCGGGTTGGACTCAAGACGATAGTTACCGGATAAGGCGCAGCGGTCGGACTGAACGGG GGGTTCGTGCATACAGTCCAGCTTGGAGCGAACTGCCTACCCGGAACTGAGTGTCAGGCGTGGAATGAGACAAACGCGGCCA TAACAGCGGAATGACACCGGTAAACCGAAAGGCAGGAACAGGAGAGCGCACGAGGGAGCCGCCAGGGGGAAACGCCTGGT ATCTTTATAGTCCTGTCGGGTTTCGCCACCACTGATTTGAGCGTCAGATTTCGTGATGCTTGTCAGGGGGCGGAGCC

$>\mathrm{pBb}$

tacggttatccacagaatcagagggcaaactactagagatGACGGGCCTGAACAAGAGCACCGTAAGCAGCCAAGTGAATACACTGATGAA GGAGTCAATGGTGTTCGAGATCGGCCAGGGCCAGAGCTCAGGTGGCCGCCGCCCGGTAATGTTAGTCTTCAACAAGAAAGCG GGCTATAGTGTGGGCATCGACGTCGGCGTAGACTACATCAACGGAATCCTTACGGATTTAGAGGGCACGATTGTGTTGGACCA GTATAGACACCTTGAGAGCAACAGCCCGGAGATCACTAAGGATATCCTTATCGACATGATCCACCATTTCATCACACAGATGCC TCAGAGCCCTTATGGATTCATCGGCATCGGCATCTGTGTACCGGGTTTAATCGACAAGGACCAGAAGATCGTGTTTACGCCTAA TAGCAATTGGCGTGACATCGATCTGAAGAGCAGCATCCAGGAGAAATATAACGTAAGCGTGTTCATCGAGAACGAAGCGAAC GCAGGAGCGTACGGCGAGAAGTTGTTCGGTGCAGCGAAGAACCATGACAATATCATCTATGTGTCAATTTCAACGGGCATCG GAATTGGCGTGATCATTAATAACCACCTGTACCGTGGCGTGTCAGGATTTAGCGGTGAGATGGGCCACATGACGATCGATTTC AACGGACCGAAGTGTTCATGTGGCAATAGAGGCTGTTGGGAGCTTTACGCGAGCGAGAAAGCGCTGCTGAAGAGCTTGCAAA CGAAGGAGAAGAAACTCAGCTACCAGGACATCATCAATCTTGCACACCTTAACGACATTGGCACACTGAACGCGCTGCAGAAC TTCGGCTTCTACCTGGGCATCGGATTGACAAACATCTTGAACACGTTCAATCCGCAGGCAGTGATCCTGCGCAACTCAATCATC GAGAGCCACCCGATGGTGCTGAACAGCATGCGCTCAGAGGTGAGCAGCCGTGTGTACAGCCAGCTCGGAAACTCATACGAGC TGCTTCCGAGCAGTCTTGGCCAGAACGCTCCTGCGCTTGGCATGAGCAGCATCGTAATCGACCACTTCTTAGATATGATCACGA TGTAATAGTCAAATGTCAGACGAAAATGCCAATTATTGAAGCGGCTAACGCCGCTTTTTTTGTTTCTGGTCTCCCCATAGTTTAA CAAGTGCTGTAAGAAGTCATTCAGCATAGCTTCTAGAGATCTGCAGTGACTGGGAAAACCCTGGCGACTAGTCTTGGACTCCT GTTGATAGATCCAGTAATGACCTCAGAACTCCATCTGGATTTGTTCAGAACGCTCGGTTGCCGCCGGGCGTTTTTTATTGGTGA GAATCCAGGGGTCCCCAATAATTACGATTTAAATCCTTCAAACTTCCCAAAGGCGAGCCCTAGTGACATTAGAAAACCGACTGT AAAAAGTACAGTCGGCATTATCTCATATTATAAAAGCCAGTCATTAGGCCTATCTGACAATTCCTGAATAGAGTTCATAAACAA TCCTGCATGATAACCATCACAAACAGAATGATGTACCTGTAAAGATAGCGGTAAATATATTGAATTACCTTTATTAATGAATTTT CCTGCTGTAATAATGGGTAGAAGGTAATTACTATTATTATTGATATTTAAGTTAAACCCAGTAAATGAAGTCCATGGAATAATA GAAAGAGAAAAAGCATTTTCAGGTATAGGTGTTTTGGGAAACAATTTCCCCGAACCATTATATTTCTCTACATCAGAAAGGTAT AAATCATAAAACTCTTTGAAGTCATTCTTTACAGGAGTCCAAATACCAGAGAATGTTTTAGATACACCATCAAAAATTGTATAAA GTGGCTCTAACTTATCCCAATAACCTAACTCTCCGTCGCTATTGTAACCAGTTCTAAAAGCTGTATTTGAGTTTATCACCCTTGTC ACTAAGAAAATAAATGCAGGGTAAAATTTATATCCTTCTTGTTTTATGTTTCGGTATAAAACACTAATATCAATTTCTGTGGTTA TACTAAAAGTCGTTTGTTGGTTCAAATAATGATTAAATATCTCTTTTCTCTTCCAATTGTCTAAATCAATTTTATTAAAGTTCATTT GATATGCCTCCTAAATTTTTATCTAAAGTGAATTTAGGAGGCTTACTTGTCTGCTTTCTTCATTAGAATCAATCCTTTTTTTAAAAG 
TCAATCCCGTTTGTTGAAGACTTTTGTCCTTTTCCGCTGCATAAACAATATGGCCCGTTTGTTGAACTACTCTTTAATAAAATAAT TTTTCCGTTCCCAATTCCACATTGCAATAATAGAAAATCCATCTTCATCGGCTTTTTTCGTCATCATCTGTATGAATCAAATCGCCT TCTTCTGTGTCATCAAGGTTTAATTTTTTATGTATTTCTTTTAACAAACCACCATAGGAGATTAACCTTTTTACGGTGTAAACCTTC CTCCAAATCAGACAAACGTTTCAAATTCTTTTCTTCATCATCGGTCATAAAATCCGTATCCTTTACAGGATATTTTGCAGTTTCGT CAATTGCCGATTGTATATCCGATTTATATTTATTTTTCGGTCGAATCATTTGAACTTTTACATTTGGATCATAGTCTAATTTCATTG CCTTTTTCCAAAATTGAATCCATTGTTTTTGATTCACGTAGTTTTTCTGTATTCTTAAAATAAGTTGGTTCCACACATACCAATACAT GCATGTGCTGATTATAAGAATTATCTTTATTATTTATTGTCACTTCCGTTGCACGCATAAAACCAACAAGATTTTTATTAATTTTT TTATATTGCATCATTCGGCGAAATCCTTGAGCCATATCTGACAAACTCTTATTTAATTCTTCGCCATCATAAACATTTTTAACTGT TAATGTGAGAAACAACCAACGAACTGTTGGCTTTTGTTTAATAACTTCAGCAACAACCTTTTGTGACTGAATGCCATGTTTCATT GCTCTCCTCCAGTTGCACATTGGACAAAGCCTGGATTTACAAAACCACACTCGATACAACTTTCTTTTCGCCTGTTTCACGATTTT GTTTATACTCTAATATTTCAGCACAATCTTTTACTCTTTCAGCCTTTTTTAAATTCAAGAATATGCAGAAGTTCAAAGTAATCAACA TTAGCGATTTTCTTTTCTCTCCATGGTCTCACTTTTTCCACTTTTTGTCTTGTCCACTAAAACCCTTGATTTTTCATCTGAATAAATGC TACTATTAGGACACATAATATTAAAAGAAACCCCCATCTATTTAGTTATTTGTTTGGTCACTTATAACTTTAACAGATGGGGTTTT TCTGTGCAACCAATTTTAAGGGTTTTCCAATACTTTAAAACACATACATACCAACACTTCAACGCACCTTTCAGCAACTAAAATA AAAATGACGTTATTTCTATATGTATCAAGATAAGAAAGAACAAGTTCAAAACCATCAAAAAAAGACACCTTTTCAGGTGCTTTT TTTATTTTTATAAACTCATTCCCTGATCTCGACTTCGTTCTTTTTTTACCTCTCGGTTATGAGTTAGTTCAAATTCGTTCTTTTTTAGGT TCTAAATCGTGTTTTTTCTTGGAATTGTGCTGTTTTATCCTTTACCTTGTCTACAAACCCCTTAAAAACGTTTTTAAAGGCTTTTAAG CGTCTGTACGTTCCTTAAGGAATTATTCCTTAGTGCTTTCTAGGTTAATGTCATGATAATAATGGTTTCTTAGACGTCAGGTGGC ACTTTTCGGGGAAATGTCCGCGGAACCCCTATTTGTTTATTTTTCTAAATACATTCAAATATGTATCCGCTCATGAGACAATAAC CCTGATAAATGCTTCAATAATATTGAAAAAGGAAGAGTATGAGTATTCAACATTTCCGTGTCGCCCTTATTCCCTTTTTTGCGGC ATTTTGCCTTCCTGTTTTTGCTCACCCAGAAACGCTGGTGAAAGTAAAAGATGCTGAAGATCAGTTGGGTGCACGAGTGGGTT ACATCGAACTGGATCTCAACAGCGGTAAGATCCTTGAGAGTTTTCGCCCCGAAGAACGTTTTCCAATGATGAGCACTTTTAAAG TTCTGCTATGTGGCGCGGTATTATCCCGTGTTGACGCCGGGCAAGAGCAACTCGGTCGCCGCATACACTATTCTCAGAATGACT TGGTTGAGTACTCACCAGTCACAGAAAAGCATCTTACGGATGGCATGACAGTAAGAGAATTATGCAGTGCTGCCATAACCATG AGTGATAACACTGCGGCCAACTTACTTCTGACAACGATCGGAGGACCGAAGGAGCTAACCGCTTTTTTGCACAACATGGGGGA TCATGTAACTCGCCTTGATCGTTGGGAACCGGAGCTGAATGAAGCCATACCAAACGACGAGCGTGACACCACGATGCCTGCAG CAATGGCAACAACGTTGCGCAAACTATTAACTGGCGAACTACTTACTCTAGCTTCCCGGCAACAATTAATAGACTGGATGGAG GCGGATAAAGTTGCAGGACCACTTCTGCGCTCGGCCCTTCCGGCTGGCTGGTTTATTGCTGATAAATCTGGAGCCGGTGAGCG TGGGTCTCGCGGTATCATTGCAGCACTGGGGCCAGATGGTAAGCCCTCCCGTATCGTAGTTATCTACACGACGGGGAGTCAGG CAACTATGGATGAACGAAATAGACAGATCGCTGAGATAGGTGCCTCACTGATTAAGCATTGGTAACTGTCAGACCAAGTTTAC TCATATATACTTTAGATTGATTTAAAACTTCATTTTTAATTTAAAAGGATCTAGGTGAAGATCCTTTTTGGATAATCTCATGACCAA AATCCCTTAACGTGAGTTTTCGTTCCACTGAGCGTCAGACCCCTTAATAAGATGATCTTCTTGAGATCGTTTTGGTCTGCGCGTA ATCTCTTGCTCTGAAAACGAAAAAACCGCCTTGCAGGGAGGTTTTTCGAAGGTTCTCTGAGCTACCAACTCTTTGAACCGAGGT AACTGGCTTGCAGGAGCGCAGTCACCAAAACTTGTCCTTTCAGTTTAGCCTTAACCGGCGCATGACTTCAAGACTAACTCCTCT AAATCAATTACCAGTGGCTGCTGCCAGTGGTGCTTTTGCATGTCTTTCCGGGTTGGACTCAAGACGATAGTTACCGGATAAGGC GCAGCGGTCGGACTGAACGGGGGGTTCGTGCATACAGTCCAGCTTGGAGCGAACTGCCTACCCGGAACTGAGTGTCAGGCGT GGAATGAGACAAACGCGGCCATAACAGCGGAATGACACCGGTAAACCGAAAGGCAGGAACAGGAGAGCGCACGAGGGAGC CGCCAGGGGGAAACGCCTGGTATCTTTATAGTCCTGTCGGGTTTCGCCACCACTGATTTGAGCGTCAGATTTCGTGATGCTTGT CAGGGGGCGGAGCCTATGGAAAAACGCTTTGCCCCACACAGGCTCTTGAACAAGGGATAACAATTTGTCATAGCTGTATTCGT TACAAGGAGGATTTTTCGAAAAAACACCCTAACGGGTGTTTTTTTATAGCTGGTCTCCCTTGCTTGTACTTTACAGTATGCCATAC GAAACTGCGTCAATACACGTTGACACTCTTTTGTAGTTATGTTAAATTATCAGATCGCCATCATTAGTTTGTTTAAACAACAAAC TAATACTAGAGGCTCTTAAAGGGGGTTTTAGATACCAGAGATGGTGAGCAAGGGCGAGGAGGATAACATGGCCATCATCAAG GAGTTCATGCGCTTCAAGGTGCACATGGAGGGCTCCGTGAACGGCCACGAGTTCGAGATCGAGGGCGAGGGCGAGGGCCGC CCCTACGAGGGCACCCAGACCGCCAAGCTGAAGGTGACCAAGGGTGGCCCCCTGCCCTTCGCCTGGGACATCCTGTCCCCTCA GTTCATGTACGGCTCCAAGGCCTACGTGAAGCACCCCGCCGACATCCCCGACTACTTGAAGCTGTCCTTCCCCGAGGGCTTCAA GTGGGAGCGCGTGATGAACTTCGAGGACGGCGGCGTGGTGACCGTGACCCAGGACTCCTCCCTGCAGGACGGCGAGTTCATC TACAAGGTGAAGCTGCGCGGCACCAACTTCCCCTCCGACGGCCCCGTAATGCAGAAGAAGACCATGGGCTGGGAGGCCTCCT CCGAGCGGATGTACCCCGAGGACGGCGCCCTGAAGGGCGAGATCAAGCAGAGGCTGAAGCTGAAGGACGGCGGCCACTAC GACGCTGAGGTCAAGACCACCTACAAGGCCAAGAAGCCCGTGCAGCTGCCCGGCGCCTACAACGTCAACATCAAGTTGGACA TCACCTCCCACAACGAGGACTACACCATCGTGGAACAGTACGAACGCGCCGAGGGCCGCCACTCCACCGGCGGCATGGACGA GCTGTACAAGTAATAGCTGCCACCTTTCTGAACTTTGGTAATGCGTCTCGGTACCAAATTCCAGAAAAGAGGCCTCCCGAAAG GGGGGCCTTTTTTCGTTTTGGTCCCTCcaatgatctccttatccggtcgacattcacgattacttggCTAGCGCTATATGCGTTGATGCAATTTC TATGCGCACCCGTTCTCGGAGCACTGTCCGACCGCTTTGGCCGCCGCCCAGTCCTGCTCGCTTCGCTACTTGGAGCCACTATCG 
ACTACGCGATCATGGCGACCACACCCGTCCTGTGGATCTATCGATGCATGCAGCCGGTACCATCACAAGCACTTTGGGACGTTC TCCCTTAGTGCTTTTTTTGATTTCTCATAGGCCGGCCTGTTAGTCATATGGACACTTAAGCTCTAGACGGTGATCAACACGCTAGC CAGGCATCAAATAAAACGAAAGGCTCAGTCGAAAGACTGGGCCTTTCGTTTTATCTGTTGTTTGTCGGTGAACGCTCTCTACTA GAGTCACACTGGCTCACCTTCGGGTGGGCCTTTCTGCGTTTATAGAATTCACTAGTAATTGTGAGGCGGATAACAATTCTCACT CATTCTACAGTTTATTCTTGACATTGCACTGTCCCCCTGGTATAATAACTAATTGTGAGCGCTCACAATTAAGATCCCCGGGGCG GCCGCGAATTCAAAAGATCTAAAGAGGAGAAAGGATCTATGGATAAGAAATACTCAATAGGCTTAGCTATCGGCACAAATAG CGTCGGATGGGCGGTGATCACTGATGAATATAAGGTTCCGTCTAAAAAGTTCAAGGTTCTGGGAAATACAGACCGCCACAGTA TCAAAAAAAATCTTATAGGGGCTCTTTTTATTTGACAGTGGAGAGACAGCGGAAGCGACTCGTCTCAAACGGACAGCTCGTAGA AGGTATACACGTCGGAAGAATCGTATTTGTTATCTACAGGAGATTTTTTCAAATGAGATGGCGAAAGTAGATGATAGTTTCTTT CATCGACTTGAAGAGTCTTTTTTGGTGGAAGAAGACAAGAAGCATGAACGTCATCCTATTTTTGGAAATATAGTAGATGAAGT TGCTTATCATGAGAAATATCCAACTATCTATCATCTGCGAAAAAAATTGGTAGATTCTACTGATAAAGCGGATTTGCGCTTAATC TATTTGGCCTTAGCGCATATGATTAAGTTTCGTGGTCATTTTTTGATTGAGGGAGATTTAAATCCTGATAATAGTGATGTGGAC AAACTATTTATCCAGTTGGTACAAACCTACAATCAATTATTTGAAGAAAACCCTATTAACGCAAGTGGAGTAGATGCTAAAGCG ATTCTTTCTGCACGATTGAGTAAATCAAGACGATTAGAAAATCTCATTGCTCAGCTCCCCGGTGAGAAGAAAAATGGCTTATTT GGGAATCTCATTGCTTTGTCATTGGGTTTGACCCCTAATTTTAAATCAAATTTTGATTTGGCAGAAGATGCTAAATTACAGCTTT CAAAAGATACTTACGATGATGATTTAGATAATTTATTGGCGCAAATTGGAGATCAATATGCTGATTTGTTTTTGGCAGCTAAGA ATTTATCAGATGCTATTTTACTTTCAGATATCCTAAGAGTAAATACTGAAATAACTAAGGCTCCCCTATCAGCTTCAATGATTAA ACGCTACGATGAACATCATCAAGACTTGACTCTTTTAAAAGCTTTAGTTCGACAACAACTTCCAGAAAAGTATAAAGAAATCTTT TTTGATCAATCAAAAAACGGATATGCAGGTTATATTGATGGGGGAGCTAGCCAAGAAGAATTTTATAAATTTATCAAACCAATT TTAGAAAAAATGGATGGTACTGAGGAATTATTGGTGAAACTAAATCGTGAAGATTTGCTGCGCAAGCAACGGACCTTTGACAA CGGCTCTATTCCCCATCAAATTCACTTGGGTGAGCTGCATGCTATTTTGAGAAGACAAGAAGACTTTTATCCATTTTTAAAAGAC AATCGTGAGAAGATTGAAAAAATCTTGACTTTTCGAATTCCTTATTATGTTGGTCCATTGGCGCGTGGCAATAGTCGTTTTGCAT GGATGACTCGGAAGTCTGAAGAAACAATTACCCCATGGAATTTTGAAGAAGTTGTCGATAAAGGTGCTTCAGCTCAATCATTT ATTGAACGCATGACAAACTTTGATAAAAATCTTCCAAATGAAAAAGTACTACCAAAACATAGTTTGCTTTATGAGTATTTTACG GTTTATAACGAATTGACAAAGGTCAAATATGTTACTGAAGGAATGCGAAAACCAGCATTTCTTTCAGGTGAACAGAAGAAAGC CATTGTTGATTTACTCTTCAAAACAAATCGAAAAGTAACCGTTAAGCAATTAAAAGAAGATTATTTCAAAAAAATAGAATGTTTT GATAGTGTTGAAATTTCAGGAGTTGAAGATAGATTTAATGCTTCATTAGGTACCTACCATGATTTGCTAAAAATTATTAAAGAT AAAGATTTTTTGGATAATGAAGAAAATGAAGATATCTTAGAGGATATTGTTTTAACATTGACCTTATTTGAAGATAGGGAGATG ATTGAGGAAAGACTTAAAACATATGCTCACCTCTTTGATGATAAGGTGATGAAACAGCTTAAACGTCGCCGTTATACTGGTTGG GGACGTTTGTCTCGAAAATTGATTAATGGTATTAGGGATAAGCAATCTGGCAAAACAATATTAGATTTTTTGAAATCAGATGGT TTTGCCAATCGCAATTTTATGCAGCTGATCCATGATGATAGTTTGACATTTAAAGAAGACATTCAAAAAGCACAAGTGTCTGGA CAAGGCGATAGTTTACATGAACATATTGCAAATTTAGCTGGTAGCCCTGCTATTAAAAAAGGTATTTTACAGACTGTAAAAGTT GTTGATGAATTGGTCAAAGTAATGGGGCGGCATAAGCCAGAAAATATCGTTATTGAAATGGCACGTGAAAATCAGACAACTC AAAAGGGCCAGAAAAATTCGCGAGAGCGTATGAAACGAATCGAAGAAGGTATCAAAGAATTAGGAAGTCAGATTCTTAAAG AGCATCCTGTTGAAAATACTCAATTGCAAAATGAAAAGCTCTATCTCTATTATCTCCAAAATGGAAGAGACATGTATGTGGACC AAGAATTAGATATTAATCGTTTAAGTGATTATGATGTCGATGCCATTGTTCCACAAAGTTTCCTTAAAGACGATTCAATAGACA ATAAGGTCTTAACGCGTTCTGATAAAAATCGTGGTAAATCGGATAACGTTCCAAGTGAAGAAGTAGTCAAAAAGATGAAAAAC TATTGGAGACAACTTCTAAACGCCAAGTTAATCACTCAACGTAAGTTTGATAATTTAACGAAAGCTGAACGTGGAGGTTTGAGT GAACTTGATAAAGCTGGTTTTATCAAACGCCAATTGGTTGAAACTCGCCAAATCACTAAGCATGTGGCACAAATTTTGGATAGT CGCATGAATACTAAATACGATGAAAATGATAAACTTATTCGAGAGGTTAAAGTGATTACCTTAAAATCTAAATTAGTTTCTGAC TTCCGAAAAGATTTCCAATTCTATAAAGTACGTGAGATTAACAATTACCATCATGCCCATGATGCGTATCTAAATGCCGTCGTTG GAACTGCTTTGATTAAGAAATATCCAAAACTTGAATCGGAGTTTGTCTATGGTGATTATAAAGTTTATGATGTTCGTAAAATGA TTGCTAAGTCTGAGCAAGAAATAGGCAAAGCAACCGCAAAATATTTCTTTTACTCTAATATCATGAACTTCTTCAAAACAGAAA TTACACTTGCAAATGGAGAGATTCGCAAACGCCCTCTAATCGAAACTAATGGGGAAACTGGAGAAATTGTCTGGGATAAAGG GCGAGATTTTGCCACAGTGCGCAAAGTATTGTCCATGCCCCAAGTCAATATTGTCAAGAAAACAGAAGTACAGACAGGCGGAT TCTCCAAGGAGTCAATTTTACCAAAAAGAAATTCGGACAAGCTTATTGCTCGTAAAAAAGACTGGGATCCAAAAAAATATGGT GGTTTTGATAGTCCAACGGTAGCTTATTCAGTCCTAGTGGTTGCTAAGGTGGAAAAAGGGAAATCGAAGAAGTTAAAATCCGT TAAAGAGTTACTAGGGATCACAATTATGGAAAGAAGTTCCTTTGAAAAAAATCCGATTGACTTTTTAGAAGCTAAAGGATATA AGGAAGTTAAAAAAGACTTAATCATTAAACTACCTAAATATAGTCTTTTTGAGTTAGAAAACGGTCGTAAACGGATGCTGGCTA GTGCCGGAGAATTACAAAAAGGAAATGAGCTGGCTCTGCCAAGCAAATATGTGAATTTTTTATATTTAGCTAGTCATTATGAAA AGTTGAAGGGTAGTCCAGAAGATAACGAACAAAAACAATTGTTTGTGGAGCAGCATAAGCATTATTTAGATGAGATTATTGAG CAAATCAGTGAATTTTCTAAGCGTGTTATTTTAGCAGATGCCAATTTAGATAAAGTTCTTAGTGCATATAACAAACATAGAGAC AAACCAATACGTGAACAAGCAGAAAATATTATTCATTTATTTACGTTGACGAATCTTGGAGCTCCCGCTGCTTTTAAATATTTTG 
ATACAACAATTGATCGTAAACGATATACGTCTACAAAAGAAGTTTTAGATGCCACTCTTATCCATCAATCCATCACTGGTCTTTA TGAAACACGCATTGATTTGAGTCAGCTAGGAGGTGACTAAGTCGACCTCGAGACTAGTCAAGGTCGGCAATTCTGCAGTACTA GGACGCCGCCAAGCCAGCTTAAACCCAGCTCAATGAGCTGGGTTTTTTTGTTTGTTAAAAATGAAGAAGAAACTGTGAAGCGTA TTTATAGCAAAGCACTCAAAAGTTTACCTTATGGGTGCTTTTTTCGTGCTTTTTTGAAAAGACAAAAAAAAGAACCTTGCCAAGC AAGATTCTTTTGATAGCGCTATCGCTGAGCGCCGGTCGCTACCATTACCAGTTGGTCTGGTGTCAAAAATAATAATAACCGGGC AGGCCATGTCTGCCCGTATTTCGCGTAAGGAAATCCATTATGTACTATTTCTGGTGATGAAATCAACGTAACATTTAAAGCTGT CAAAGCCAAAGTCATGAGATGGCGTATGGAGCGTAAAGCTGACAAGAGCGGTGTTGCGATGATTGAGATGACCTTCCTTGCA CCAAGTGAATTGCCTCAAGAAAGCACTCAATCAAAGATTCTTGTAGATGGAAAAGAACTTGCTGATTTCGCTGAAAATCGTCAA GACTATCAAATTACCTATAAAGGTCAACGGCCAAAAGTCTCAGTTGAAGAAAACAATCAAGTAGCTTCAACTGTGGTAGATAG TGGAGAAGATAGCCTTCCAGTACTTGTTCGCCTCGTTTCAGAAAGTGGAAAACAAGTCAAGGAATACCGTATCCAGTTGACTA AGGAAAAACCAGTTTCTGCTGTACAAGAAGATCTTCCAAAACTCGAATTTGTTGAAAAAGATTTGGCCTACAAGACAGTTGAG AAAAAAGATTCAACACTGTATCTAGGTGAAACTCGTGTAGAACAAGAAGGAAAAGTTGGAAAAGAACGTATCTTTACAGTGAT TAATCCTGATGGAAGTAAGGAAGAAAAACTCCGTGAAGTGGTAGAAGTTCCGACAGACCGCATCGTCTTGGTTGGAACCAAA CCAGTAGCTCAAGAAGCTAAAAAACCACAAGTGTCAGAAAAAGCAGATACAAAACCAATTGATTCAAGTGAAGCTGATCAAAC TAATAAAGCCCAGTTACCAAATACAGGTAGTGCGGCAAGCCAAGCAGCAGTAGCAGCAGGTTTAGCCTGCCTCGCGCGTTTCG GTGATGACGGTGAAAACCTCTGACACATGCAGCTCCCGGAGACGGTCACAGCTTGTCTGTAAGCGGATGCCGGGAGCAGACA AGCCCGTCAGGGCGCGTCAGCGGGTGTTGGCGGGTGTCGGGGCGCAGCCATGACCCAGTCACGTAGCGATAGCGGAGTGTA TACTGGCTTAACTATGCGGCATCAGAGCAGATTGTACTGAGAGTGCACCATATGCGGTGTGAAATACCGCACAGATGCGTAAG GAGAAAATACCGCATCAGGCGCTCTTCCGCTTCCTCGCTCACTGACTCGCTGCGCTCGGTCGTTCGGCTGCGGCGAGCGGTAT CAGCTCACTCAAAGGCGGTAA

$>$ pV1-PsspA

GAGCCTATGGAAAAACGCTTTGCCCCACACAGGCTCTTGAACAAGGGATAACAATTTGTCATAGCTGTATTCGTTACAAGGAG GATTTTCGAAAAAACACCCTAACGGGTGTTTTTTTATAGCTGGTCTCCCCAAGTGCTGTAAAAAACGCCGAATGAAGCGGATG AAATTGCTCATCGTCTCGTGAAAAAGCCGAAGAAGGTTAAGCCTGGGTATAAAAAGAAAATGAGCTATGAGATGGAGAAAGC CATAACCATGAGTGATAACACTGGTTTTAGAGCTAGAAATAGCAAGTTAAAATAAGGCTAGTCCGTTATCAACTTGAAAAAGT GGCACCGAGTCGGTGCTTTTTTTCTCGGTACCAAATTCCAGAAAAGAGGCCTCCCGAAAGGGGGGCCTTTTTTTCGTTTTGGTCC CTCAATGATCTCCTTATCCGGTTAAGATGGCAAGCTTGACAAGTATTTCTTCAGAATTTACAATGAAGTTTACATGACGCTCTTA AAGGGGGTTTTAGATACCAGAGATGGATAAGAAATACTCAATAGGCTTAGATATCGGCACAAATAGCGTCGGATGGGCGGTG AAACACGCATTGATTTGAGTCAGCTAGGAGGTGACTGATAATCAAATGTCAGACGAAAATGCCAATTATTGAAGCGGCTAACG CCGCTTTTTTTGTTTCTGGTCTCCCTTGCTTGTACTTTACAGTATAGCTTCTAGAGATCTGCAGTGACTGGAGCTTCTAGAGATCT GCAGTGACTGGGAAAACCCTGGCGACTAGTCTTGGACTCCTGTTGATAGATCCAGTAATGACCTCAGAACTCCATCTGGATTTG TTCAGAACGCTCGGTTGCCGCCGGGCGTTTTTTATTGGTGAGAATCCAGGGGTCCCCAATAATTACGATTTAAATCCTTCAAAC TTCCCAAAGGCGAGCCCTAGTGACATTAGAAAACCGACTGTAAAAAGTACAGTCGGCATTATCTCATATTATAAAAGCCAGTCA TTAGGCCTATCTGACAATTCCTGAATAGAGTTCATAAACAATCCTGCATGATAACCATCACAAACAGAATGATGTACCTGTAAA GATAGCGGTAAATATATTGAATTACCTTTATTAATGAATTTTCCTGCTGTAATAATGGGTAGAAGGTAATTACTATTATTATTGA TATTTAAGTTAAACCCAGTAAATGAAGTCCATGGAATAATAGAAAGAGAAAAAGCATTTTCAGGTATAGGTGTTTTGGGAAAC AATTTCCCCGAACCATTATATTTCTCTACATCAGAAAGGTATAAATCATAAAACTCTTTGAAGTCATTCTTTACAGGAGTCCAAA TACCAGAGAATGTTTTAGATACACCATCAAAAATTGTATAAAGTGGCTCTAACTTATCCCAATAACCTAACTCTCCGTCGCTATT GTAACCAGTTCTAAAAGCTGTATTTGAGTTTATCACCCTTGTCACTAAGAAAATAAATGCAGGGTAAAATTTATATCCTTCTTGT TTTATGTTTCGGTATAAAACACTAATATCAATTTCTGTGGTTATACTAAAAGTCGTTTGTTGGTTCAAATAATGATTAAATATCTC TTTTCTCTTCCAATTGTCTAAATCAATTTTATTAAAGTTCATTTGATATGCCTCCTAAATTTTTATCTAAAGTGAATTTAGGAGGCT TACTTGTCTGCTTTCTTCATTAGAATCAATCCTTTTTTAAAAGTCAATCCCGTTTGTTGAAGACTTTTGTCCTTTTCCGCTGCATAA ACAATATGGCCCGTTTGTTGAACTACTCTTTAATAAAATAATTTTTCCGTTCCCAATTCCACATTGCAATAATAGAAAATCCATCT TCATCGGCTTTTTTCGTCATCATCTGTATGAATCAAATCGCCTTCTTCTGTGTCATCAAGGTTTAATTTTTTTATGTATTTCTTTTAAC AAACCACCATAGGAGATTAACCTTTTACGGTGTAAACCTTCCTCCAAATCAGACAAACGTTTCAAATTCTTTTCTTCATCATCGG TCATAAAATCCGTATCCTTTACAGGATATTTTGCAGTTTCGTCAATTGCCGATTGTATATCCGATTTATATTTATTTTTCGGTCGA ATCATTTGAACTTTTACATTTGGATCATAGTCTAATTTCATTGCCTTTTTCCAAAATTGAATCCATTGTTTTTGATTCACGTAGTTT TCTGTATTCTTAAAATAAGTTGGTTCCACACATACCAATACATGCATGTGCTGATTATAAGAATTATCTTTATTATTTATTGTCAC TTCCGTTGCACGCATAAAACCAACAAGATTTTTATTAATTTTTTTATATTGCATCATTCGGCGAAATCCTTGAGCCATATCTGACA AACTCTTATTTAATTCTTCGCCATCATAAACATTTTTAACTGTTAATGTGAGAAACAACCAACGAACTGTTGGCTTTTGTTTAATA ACTTCAGCAACAACCTTTTGTGACTGAATGCCATGTTTCATTGCTCTCCTCCAGTTGCACATTGGACAAAGCCTGGATTTACAAA ACCACACTCGATACAACTTTCTTTTCGCCTGTTTCACGATTTTGTTTATACTCTAATATTTCAGCACAATCTTTTTACTCTTTCAGCCT 
TTTTAAATTCAAGAATATGCAGAAGTTCAAAGTAATCAACATTAGCGATTTTCTTTTCTCTCCATGGTCTCACTTTTCCACTTTTT GTCTTGTCCACTAAAACCCTTGATTTTTCATCTGAATAAATGCTACTATTAGGACACATAATATTAAAAGAAACCCCCATCTATTT AGTTATTTGTTTGGTCACTTATAACTTTAACAGATGGGGTTTTTCTGTGCAACCAATTTTAAGGGTTTTCCAATACTTTAAAACAC ATACATACCAACACTTCAACGCACCTTTCAGCAACTAAAATAAAAATGACGTTATTTCTATATGTATCAAGATAAGAAAGAACA AGTTCAAAACCATCAAAAAAAGACACCTTTTCAGGTGCTTTTTTTATTTTTATAAACTCATTCCCTGATCTCGACTTCGTTCTTTTTT TACCTCTCGGTTATGAGTTAGTTCAAATTCGTTCTTTTTAGGTTCTAAATCGTGTTTTTTCTTGGAATTGTGCTGTTTTATCCTTTAC CTTGTCTACAAACCCCTTAAAAACGTTTTTAAAGGCTTTTAAGCGTCTGTACGTTCCTTAAGGAATTATTCCTTAGTGCTTTCTAG GTTAATGTCATGATAATAATGGTTTCTTAGACGTCAGGTGGCACTTTTCGGGGAAATGTCCGCGGAACCCCTATTTGTTTATTTT TCTAAATACATTCAAATATGTATCCGCTCATGAGACAATAACCCTGATAAATGCTTCAATAATATTGAAAAAGGAAGAGTATGA GTATTCAACATTTCCGTGTCGCCCTTATTCCCTTTTTTGCGGCATTTTGCCTTCCTGTTTTTGCTCACCCAGAAACGCTGGTGAAA GTAAAAGATGCTGAAGATCAGTTGGGTGCACGAGTGGGTTACATCGAACTGGATCTCAACAGCGGTAAGATCCTTGAGAGTT TTCGCCCCGAAGAACGTTTTCCAATGATGAGCACTTTTAAAGTTCTGCTATGTGGCGCGGTATTATCCCGTGTTGACGCCGGGC AAGAGCAACTCGGTCGCCGCATACACTATTCTCAGAATGACTTGGTTGAGTACTCACCAGTCACAGAAAAGCATCTTACGGAT GGCATGACAGTAAGAGAATTATGCAGTGCTGCCATAACCATGAGTGATAACACTGCGGCCAACTTACTTCTGACAACGATCGG AGGACCGAAGGAGCTAACCGCTTTTTTGCACAACATGGGGGATCATGTAACTCGCCTTGATCGTTGGGAACCGGAGCTGAATG AAGCCATACCAAACGACGAGCGTGACACCACGATGCCTGCAGCAATGGCAACAACGTTGCGCAAACTATTAACTGGCGAACTA CTTACTCTAGCTTCCCGGCAACAATTAATAGACTGGATGGAGGCGGATAAAGTTGCAGGACCACTTCTGCGCTCGGCCCTTCCG GCTGGCTGGTTTATTGCTGATAAATCTGGAGCCGGTGAGCGTGGGTCTCGCGGTATCATTGCAGCACTGGGGCCAGATGGTA AGCCCTCCCGTATCGTAGTTATCTACACGACGGGGAGTCAGGCAACTATGGATGAACGAAATAGACAGATCGCTGAGATAGG TGCCTCACTGATTAAGCATTGGTAACTGTCAGACCAAGTTTACTCATATATACTTTAGATTGATTTAAAACTTCATTTTTAATTTA AAAGGATCTAGGTGAAGATCCTTTTTGATAATCTCATGACCAAAATCCCTTAACGTGAGTTTTCGTTCCACTGAGCGTCAGACC CCTTAATAAGATGATCTTCTTGAGATCGTTTTGGTCTGCGCGTAATCTCTTGCTCTGAAAACGAAAAAACCGCCTTGCAGGGAG GTTTTTCGAAGGTTCTCTGAGCTACCAACTCTTTGAACCGAGGTAACTGGCTTGCAGGAGCGCAGTCACCAAAACTTGTCCTTT CAGTTTAGCCTTAACCGGCGCATGACTTCAAGACTAACTCCTCTAAATCAATTACCAGTGGCTGCTGCCAGTGGTGCTTTTGCAT GTCTTTCCGGGTTGGACTCAAGACGATAGTTACCGGATAAGGCGCAGCGGTCGGACTGAACGGGGGGTTCGTGCATACAGTC CAGCTTGGAGCGAACTGCCTACCCGGAACTGAGTGTCAGGCGTGGAATGAGACAAACGCGGCCATAACAGCGGAATGACACC GGTAAACCGAAAGGCAGGAACAGGAGAGCGCACGAGGGAGCCGCCAGGGGGAAACGCCTGGTATCTTTATAGTCCTGTCGG GTTTCGCCACCACTGATTTGAGCGTCAGATTTCGTGATGCTTGTCAGGGGGCGGAGCCTATGGAAAAACGCTTTGCCC

\section{$>$ pCas9g1-cons}

aataggcttagatatcggcacaaatagcgtcggatgggCGGTGATCACTGATGAATATAAGGTTCCGTCTAAAAAGTTCAAGGTTCTGGGA AATACAGACCGCCACAGTATCAAAAAAATCTTATAGGGGCTCTTTTATTTGACAGTGGAGAGACAGCGGAAGCGACTCGTCT CAAACGGACAGCTCGTAGAAGGTATACACGTCGGAAGAATCGTATTTGTTATCTACAGGAGATTTTTTCAAATGAGATGGCGA AAGTAGATGATAGTTTCTTTCATCGACTTGAAGAGTCTTTTTTGGTGGAAGAAGACAAGAAGCATGAACGTCATCCTATTTTTG GAAATATAGTAGATGAAGTTGCTTATCATGAGAAATATCCAACTATCTATCATCTGCGAAAAAAATTGGTAGATTCTACTGATA AAGCGGATTTGCGCTTAATCTATTTGGCCTTAGCGCATATGATTAAGTTTCGTGGTCATTTTTTGATTGAGGGAGATTTAAATCC TGATAATAGTGATGTGGACAAACTATTTATCCAGTTGGTACAAACCTACAATCAATTATTTGAAGAAAACCCTATTAACGCAAG TGGAGTAGATGCTAAAGCGATTCTTTCTGCACGATTGAGTAAATCAAGACGATTAGAAAATCTCATTGCTCAGCTCCCCGGTGA GAAGAAAAATGGCTTATTTGGGAATCTCATTGCTTTGTCATTGGGTTTGACCCCTAATTTTAAATCAAATTTTGATTTGGCAGAA GATGCTAAATTACAGCTTTCAAAAGATACTTACGATGATGATTTAGATAATTTATTGGCGCAAATTGGAGATCAATATGCTGAT TTGTTTTTGGCAGCTAAGAATTTATCAGATGCTATTTTACTTTCAGATATCCTAAGAGTAAATACTGAAATAACTAAGGCTCCCC TATCAGCTTCAATGATTAAACGCTACGATGAACATCATCAAGACTTGACTCTTTTAAAAGCTTTAGTTCGACAACAACTTCCAGA AAAGTATAAAGAAATCTTTTTTGATCAATCAAAAAACGGATATGCAGGTTATATTGATGGGGGAGCTAGCCAAGAAGAATTTT ATAAATTTATCAAACCAATTTTAGAAAAAATGGATGGTACTGAGGAATTATTGGTGAAACTAAATCGTGAAGATTTGCTGCGC AAGCAACGGACCTTTGACAACGGCTCTATTCCCCATCAAATTCACTTGGGTGAGCTGCATGCTATTTTGAGAAGACAAGAAGA CTTTTATCCATTTTTAAAAGACAATCGTGAGAAGATTGAAAAAATCTTGACTTTTCGAATTCCTTATTATGTTGGTCCATTGGCG CGTGGCAATAGTCGTTTTGCATGGATGACTCGGAAGTCTGAAGAAACAATTACCCCATGGAATTTTGAAGAAGTTGTCGATAA AGGTGCTTCAGCTCAATCATTTATTGAACGCATGACAAACTTTGATAAAAATCTTCCAAATGAAAAAGTACTACCAAAACATAG TTTGCTTTATGAGTATTTTACGGTTTATAACGAATTGACAAAGGTCAAATATGTTACTGAAGGAATGCGAAAACCAGCATTTCTT TCAGGTGAACAGAAGAAAGCCATTGTTGATTTACTCTTCAAAACAAATCGAAAAGTAACCGTTAAGCAATTAAAAGAAGATTA TTTCAAAAAAATAGAATGTTTTGATAGTGTTGAAATTTCAGGAGTTGAAGATAGATTTAATGCTTCATTAGGTACCTACCATGA TTTGCTAAAAATTATTAAAGATAAAGATTTTTTGGATAATGAAGAAAATGAAGATATCTTAGAGGATATTGTTTTAACATTGAC CTTATTTGAAGATAGGGAGATGATTGAGGAAAGACTTAAAACATATGCTCACCTCTTTGATGATAAGGTGATGAAACAGCTTA 
AACGTCGCCGTTATACTGGTTGGGGACGTTTGTCTCGAAAATTGATTAATGGTATTAGGGATAAGCAATCTGGCAAAACAATA TTAGATTTTTTGAAATCAGATGGTTTTGCCAATCGCAATTTTATGCAGCTGATCCATGATGATAGTTTGACATTTAAAGAAGACA TTCAAAAAGCACAAGTGTCTGGACAAGGCGATAGTTTACATGAACATATTGCAAATTTAGCTGGTAGCCCTGCTATTAAAAAA GGTATTTTACAGACTGTAAAAGTTGTTGATGAATTGGTCAAAGTAATGGGGCGGCATAAGCCAGAAAATATCGTTATTGAAAT GGCACGTGAAAATCAGACAACTCAAAAGGGCCAGAAAAATTCGCGAGAGCGTATGAAACGAATCGAAGAAGGTATCAAAGA ATTAGGAAGTCAGATTCTTAAAGAGCATCCTGTTGAAAATACTCAATTGCAAAATGAAAAGCTCTATCTCTATTATCTCCAAAAT GGAAGAGACATGTATGTGGACCAAGAATTAGATATTAATCGTTTAAGTGATTATGATGTCGATCACATTGTTCCACAAAGTTTC CTTAAAGACGATTCAATAGACAATAAGGTCTTAACGCGTTCTGATAAAAATCGTGGTAAATCGGATAACGTTCCAAGTGAAGA AGTAGTCAAAAAGATGAAAAACTATTGGAGACAACTTCTAAACGCCAAGTTAATCACTCAACGTAAGTTTGATAATTTAACGAA AGCTGAACGTGGAGGTTTGAGTGAACTTGATAAAGCTGGTTTTATCAAACGCCAATTGGTTGAAACTCGCCAAATCACTAAGC ATGTGGCACAAATTTTGGATAGTCGCATGAATACTAAATACGATGAAAATGATAAACTTATTCGAGAGGTTAAAGTGATTACCT TAAAATCTAAATTAGTTTCTGACTTCCGAAAAGATTTCCAATTCTATAAAGTACGTGAGATTAACAATTACCATCATGCCCATGA TGCGTATCTAAATGCCGTCGTTGGAACTGCTTTGATTAAGAAATATCCAAAACTTGAATCGGAGTTTGTCTATGGTGATTATAA AGTTTATGATGTTCGTAAAATGATTGCTAAGTCTGAGCAAGAAATAGGCAAAGCAACCGCAAAATATTTCTTTTACTCTAATAT CATGAACTTCTTCAAAACAGAAATTACACTTGCAAATGGAGAGATTCGCAAACGCCCTCTAATCGAAACTAATGGGGAAACTG GAGAAATTGTCTGGGATAAAGGGCGAGATTTTGCCACAGTGCGCAAAGTATTGTCCATGCCCCAAGTCAATATTGTCAAGAAA ACAGAAGTACAGACAGGCGGATTCTCCAAGGAGTCAATTTTACCAAAAAGAAATTCGGACAAGCTTATTGCTCGTAAAAAAGA CTGGGATCCAAAAAAATATGGTGGTTTTGATAGTCCAACGGTAGCTTATTCAGTCCTAGTGGTTGCTAAGGTGGAAAAAGGGA AATCGAAGAAGTTAAAATCCGTTAAAGAGTTACTAGGGATCACAATTATGGAAAGAAGTTCCTTTGAAAAAAATCCGATTGAC TTTTTAGAAGCTAAAGGATATAAGGAAGTTAAAAAAGACTTAATCATTAAACTACCTAAATATAGTCTTTTTGAGTTAGAAAAC GGTCGTAAACGGATGCTGGCTAGTGCCGGAGAATTACAAAAAGGAAATGAGCTGGCTCTGCCAAGCAAATATGTGAATTTTTT ATATTTAGCTAGTCATTATGAAAAGTTGAAGGGTAGTCCAGAAGATAACGAACAAAAACAATTGTTTGTGGAGCAGCATAAGC ATTATTTAGATGAGATTATTGAGCAAATCAGTGAATTTTCTAAGCGTGTTATTTTAGCAGATGCCAATTTAGATAAAGTTCTTAG TGCATATAACAAACATAGAGACAAACCAATACGTGAACAAGCAGAAAATATTATTCATTTATTTACGTTGACGAATCTTGGAGC TCCCGCTGCTTTTAAATATTTTGATACAACAATTGATCGTAAACGATATACGTCTACAAAAGAAGTTTTAGATGCCACTCTTATC CATCAATCCATCACTGGTCTTTATgaaacacgcattgatttgagtcagctaggaggtgactgaTAATCAAATGTCAGACGAAAATGCCAATTA TTGAAGCGGCTAACGCCGCTTTTTTTGTTTCTGGTCTCCCTTGCTTGTACTTTACAGTATAGCTTCTAGAGATCTGCAGTGACTG GAGCTTCTAGAGATCTGCAGTGACTGGGAAAACCCTGGCGACTAGTCTTGGACTCCTGTTGATAGATCCAGTAATGACCTCAG AACTCCATCTGGATTTGTTCAGAACGCTCGGTTGCCGCCGGGCGTTTTTTATTGGTGAGAATCCAGGGGTCCCCAATAATTACG ATTTAAATCCTTCAAACTTCCCAAAGGCGAGCCCTAGTGACATTAGAAAACCGACTGTAAAAAGTACAGTCGGCATTATCTCAT ATTATAAAAGCCAGTCATTAGGCCTATCTGACAATTCCTGAATAGAGTTCATAAACAATCCTGCATGATAACCATCACAAACAG AATGATGTACCTGTAAAGATAGCGGTAAATATATTGAATTACCTTTATTAATGAATTTTCCTGCTGTAATAATGGGTAGAAGGT AATTACTATTATTATTGATATTTAAGTTAAACCCAGTAAATGAAGTCCATGGAATAATAGAAAGAGAAAAAGCATTTTCAGGTA TAGGTGTTTTGGGAAACAATTTCCCCGAACCATTATATTTCTCTACATCAGAAAGGTATAAATCATAAAACTCTTTGAAGTCATT CTTTACAGGAGTCCAAATACCAGAGAATGTTTTAGATACACCATCAAAAATTGTATAAAGTGGCTCTAACTTATCCCAATAACCT AACTCTCCGTCGCTATTGTAACCAGTTCTAAAAGCTGTATTTGAGTTTATCACCCTTGTCACTAAGAAAATAAATGCAGGGTAAA ATTTATATCCTTCTTGTTTTATGTTTCGGTATAAAACACTAATATCAATTTCTGTGGTTATACTAAAAGTCGTTTGTTGGTTCAAA TAATGATTAAATATCTCTTTTCTCTTCCAATTGTCTAAATCAATTTTATTAAAGTTCATTTGATATGCCTCCTAAATTTTTATCTAAA GTGAATTTAGGAGGCTTACTTGTCTGCTTTCTTCATTAGAATCAATCCTTTTTTAAAAGTCAATCCCGTTTGTTGAAGACTTTTGT CCTTTTCCGCTGCATAAACAATATGGCCCGTTTGTTGAACTACTCTTTAATAAAATAATTTTTCCGTTCCCAATTCCACATTGCAA TAATAGAAAATCCATCTTCATCGGCTTTTTCGTCATCATCTGTATGAATCAAATCGCCTTCTTCTGTGTCATCAAGGTTTAATTTT TTATGTATTTCTTTTAACAAACCACCATAGGAGATTAACCTTTTACGGTGTAAACCTTCCTCCAAATCAGACAAACGTTTCAAATT CTTTTCTTCATCATCGGTCATAAAATCCGTATCCTTTACAGGATATTTTGCAGTTTCGTCAATTGCCGATTGTATATCCGATTTAT ATTTATTTTTCGGTCGAATCATTTGAACTTTTACATTTGGATCATAGTCTAATTTCATTGCCTTTTTTCCAAAATTGAATCCATTGTT TTTGATTCACGTAGTTTTCTGTATTCTTAAAATAAGTTGGTTCCACACATACCAATACATGCATGTGCTGATTATAAGAATTATCT TTATTATTTATTGTCACTTCCGTTGCACGCATAAAACCAACAAGATTTTTATTAATTTTTTTATATTGCATCATTCGGCGAAATCCT TGAGCCATATCTGACAAACTCTTATTTAATTCTTCGCCATCATAAACATTTTTAACTGTTAATGTGAGAAACAACCAACGAACTG TTGGCTTTTGTTTAATAACTTCAGCAACAACCTTTTGTGACTGAATGCCATGTTTCATTGCTCTCCTCCAGTTGCACATTGGACAA AGCCTGGATTTACAAAACCACACTCGATACAACTTTCTTTTGCCTGTTTCACGATTTTGTTTATACTCTAATATTTCAGCACAATC TTTTACTCTTTCAGCCTTTTTAAATTCAAGAATATGCAGAAGTTCAAAGTAATCAACATTAGCGATTTTCTTTTCTCTCCATGGTC TCACTTTTCCACTTTTTGTCTTGTCCACTAAAACCCTTGATTTTTTCATCTGAATAAATGCTACTATTAGGACACATAATATTAAAA GAAACCCCCATCTATTTAGTTATTTGTTTGGTCACTTATAACTTTAACAGATGGGGTTTTTCTGTGCAACCAATTTTAAGGGTTTT CCAATACTTTAAAACACATACATACCAACACTTCAACGCACCTTTCAGCAACTAAAATAAAAATGACGTTATTTCTATATGTATC 
AAGATAAGAAAGAACAAGTTCAAAACCATCAAAAAAAGACACCTTTTCAGGTGCTTTTTTTATTTTTATAAACTCATTCCCTGATC TCGACTTCGTTCTTTTTTTTACCTCTCGGTTATGAGTTAGTTCAAATTCGTTCTTTTTTAGGTTCTAAATCGTGTTTTTCTTGGAATTG TGCTGTTTTATCCTTTAACCTTGTCTACAAACCCCTTAAAAACGTTTTTAAAGGCTTTTAAGCGTCTGTACGTTCCTTAAGGAATTA TTCCTTAGTGCTTTCTAGGTTAATGTCATGATAATAATGGTTTCTTAGACGTCAGGTGGCACTTTTCGGGGAAATGTCCGCGGA ACCCCTATTTGTTTATTTTTCTAAATACATTCAAATATGTATCCGCTCATGAGACAATAACCCTGATAAATGCTTCAATAATATTG AAAAAGGAAGAGTATGAGTATTCAACATTTCCGTGTCGCCCTTATTCCCTTTTTTGGGGCATTTTGCCTTCCTGTTTTTGCTCACC CAGAAACGCTGGTGAAAGTAAAAGATGCTGAAGATCAGTTGGGTGCACGAGTGGGTTACATCGAACTGGATCTCAACAGCGG TAAGATCCTTGAGAGTTTTTCGCCCCGAAGAACGTTTTCCAATGATGAGCACTTTTAAAGTTCTGCTATGTGGCGCGGTATTATC CCGTGTTGACGCCGGGCAAGAGCAACTCGGTCGCCGCATACACTATTCTCAGAATGACTTGGTTGAGTACTCACCAGTCACAG AAAAGCATCTTACGGATGGCATGACAGTAAGAGAATTATGCAGTGCTGCCATAACCATGAGTGATAACACTGCGGCCAACTTA CTTCTGACAACGATCGGAGGACCGAAGGAGCTAACCGCTTTTTTGCACAACATGGGGGATCATGTAACTCGCCTTGATCGTTG GGAACCGGAGCTGAATGAAGCCATACCAAACGACGAGCGTGACACCACGATGCCTGCAGCAATGGCAACAACGTTGCGCAAA CTATTAACTGGCGAACTACTTACTCTAGCTTCCCGGCAACAATTAATAGACTGGATGGAGGCGGATAAAGTTGCAGGACCACT TCTGCGCTCGGCCCTTCCGGCTGGCTGGTTTATTGCTGATAAATCTGGAGCCGGTGAGCGTGGGTCTCGCGGTATCATTGCAG CACTGGGGCCAGATGGTAAGCCCTCCCGTATCGTAGTTATCTACACGACGGGGAGTCAGGCAACTATGGATGAACGAAATAG ACAGATCGCTGAGATAGGTGCCTCACTGATTAAGCATTGGTAACTGTCAGACCAAGTTTACTCATATATACTTTAGATTGATTTA AAACTTCATTTTTAATTTAAAAGGATCTAGGTGAAGATCCTTTTTTGATAATCTCATGACCAAAATCCCTTAACGTGAGTTTTCGTT CCACTGAGCGTCAGACCCCTTAATAAGATGATCTTCTTGAGATCGTTTTGGTCTGCGCGTAATCTCTTGCTCTGAAAACGAAAA AACCGCCTTGCAGGGAGGTTTTTTCGAAGGTTCTCTGAGCTACCAACTCTTTGAACCGAGGTAACTGGCTTGCAGGAGCGCAGT CACCAAAACTTGTCCTTTCAGTTTAGCCTTAACCGGCGCATGACTTCAAGACTAACTCCTCTAAATCAATTACCAGTGGCTGCTG CCAGTGGTGCTTTTGCATGTCTTTCCGGGTTGGACTCAAGACGATAGTTACCGGATAAGGCGCAGCGGTCGGACTGAACGGG GGGTTCGTGCATACAGTCCAGCTTGGAGCGAACTGCCTACCCGGAACTGAGTGTCAGGCGTGGAATGAGACAAACGCGGCCA TAACAGCGGAATGACACCGGTAAACCGAAAGGCAGGAACAGGAGAGCGCACGAGGGAGCCGCCAGGGGGAAACGCCTGGT ATCTTTATAGTCCTGTCGGGTTTCGCCACCACTGATTTGAGCGTCAGATTTCGTGATGCTTGTCAGGGGGCGGAGCCTATGGAA AAACGCTTTGCCCGAGCCTATGGAAAAACGCTTTGCCCCACACAGGCTCTTGAACAAGGGATAACAATTTGTCATAGCTGTATT CGTTACAAGGAGGATTTTCGAAAAAACACCCTAACGGGTGTTTTTTTATAGCTGGTCTCCCCAAGTGCTGTAAAAAACGCCGAA TGAAGCGGATGAAATTGCTCATCGTCTCGTGAAAAAGCCGAAGAAGGTTAAGCCTGGGTATAAAAAGAAAATGAGCTATGAG ATGGAGAAAGCCATAACCATGAGTGATAACACTGGTTTTAGAGCTAGAAATAGCAAGTTAAAATAAGGCTAGTCCGTTATCAA CTTGAAAAAGTGGCACCGAGTCGGTGCTTTTTTTCTCGGTACCAAATTCCAGAAAAGAGGCCTCCCGAAAGGGGGGCCTTTTTT CGTTTTGGTCCCTCAATGATCTCCTTATCCGGTTAAGATGGCAAGCTTGACAAGTATTTCTTCAGAATTTACAATGAAGTTTACA TGACGCTCTTAAAGGGGGTTTTAGATACCAGAGATGGATAAGAAATACTC

$>$ pCas9g2-cons AGGTCAGACGCGATTTCCTGGGTGCTCGAGCCATGGGAAAGCGGTGGTGGAAAAAACGCCGAATGAAGCGGATGAAATTGC TCATCGTCTCGTGAAAAAGCCGAAGAAGGTTAAGCCTGGGTATAAAAAGAAAATGAGCTATGAGATGGAGAAATTGAAGTCA TGCGCCGGTTAGTTTTAGAGCTAGAAATAGCAAGTTAAAATAAGGCTAGTCCGTTATCAACTTGAAAAAGTGGCACCGAGTCG GTGCTTTTTTTCTCGGTACCAAATTCCAGAAAAGAGGCCTCCCGAAAGGGGGGCCTTTTTTCGTTTTGGTCCCTCAATGATCCG ACAGGAAGTGGTATTAGCGACTCCTACAAGGAATAGGTGTCTGGGTCATGCAGTGACTGGGAAAACCCTGGCGACTAGTCTT GGACTCCTGTTGATAGATCCAGTAATGACCTCAGAACTCCATCTGGATTTGTTCAGAACGCTCGGTTGCCGCCGGGCGTTTTTT ATTGGTGAGAATCCAGGGGTCCCCAATAATTACGATTTAAATCCTTCAAACTTCCCAAAGGCGAGCCCTAGTGACATTAGAAAA CCGACTGTAAAAAGTACAGTCGGCATTATCTCATATTATAAAAGCCAGTCATTAGGCCTATCTGACAATTCCTGAATAGAGTTC ATAAACAATCCTGCATGATAACCATCACAAACAGAATGATGTACCTGTAAAGATAGCGGTAAATATATTGAATTACCTTTATTA ATGAATTTTCCTGCTGTAATAATGGGTAGAAGGTAATTACTATTATTATTGATATTTAAGTTAAACCCAGTAAATGAAGTCCATG GAATAATAGAAAGAGAAAAAGCATTTTTCAGGTATAGGTGTTTTGGGAAACAATTTCCCCGAACCATTATATTTCTCTACATCAG AAAGGTATAAATCATAAAACTCTTTGAAGTCATTCTTTTACAGGAGTCCAAATACCAGAGAATGTTTTAGATACACCATCAAAAA TTGTATAAAGTGGCTCTAACTTATCCCAATAACCTAACTCTCCGTCGCTATTGTAACCAGTTCTAAAAGCTGTATTTGAGTTTATC ACCCTTGTCACTAAGAAAATAAATGCAGGGTAAAATTTATATCCTTCTTGTTTTATGTTTCGGTATAAAACACTAATATCAATTTC TGTGGTTATACTAAAAGTCGTTTGTTGGTTCAAATAATGATTAAATATCTCTTTTCTCTTCCAATTGTCTAAATCAATTTTATTAA AGTTCATTTGATATGCCTCCTAAATTTTTATCTAAAGTGAATTTAGGAGGCTTACTTGTCTGCTTTCTTCATTAGAATCAATCCTT TTTTAAAAGTCAATCCCGTTTGTTGAAGACTTTTGTCCTTTTCCGCTGCATAAACAATATGGCCCGTTTGTTGAACTACTCTTTAA TAAAATAATTTTTCCGTTCCCAATTCCACATTGCAATAATAGAAAATCCATCTTCATCGGCTTTTTCGTCATCATCTGTATGAATC AAATCGCCTTCTTCTGTGTCATCAAGGTTTAATTTTTTATGTATTTCTTTTAACAAACCACCATAGGAGATTAACCTTTTACGGTG TAAACCTTCCTCCAAATCAGACAAACGTTTCAAATTCTTTTCTTCATCATCGGTCATAAAATCCGTATCCTTTACAGGATATTTTG 
CAGTTTCGTCAATTGCCGATTGTATATCCGATTTATATTTATTTTTCGGTCGAATCATTTGAACTTTTACATTTGGATCATAGTCT AATTTCATTGCCTTTTTTCCAAAATTGAATCCATTGTTTTTGATTCACGTAGTTTTCTGTATTCTTAAAATAAGTTGGTTCCACACAT ACCAATACATGCATGTGCTGATTATAAGAATTATCTTTATTATTTATTGTCACTTCCGTTGCACGCATAAAACCAACAAGATTTTT ATTAATTTTTTTTATATTGCATCATTCGGCGAAATCCTTGAGCCATATCTGACAAACTCTTATTTAATTCTTCGCCATCATAAACATT TTTAACTGTTAATGTGAGAAACAACCAACGAACTGTTGGCTTTTGTTTAATAACTTCAGCAACAACCTTTTGTGACTGAATGCCA TGTTTCATTGCTCTCCTCCAGTTGCACATTGGACAAAGCCTGGATTTACAAAACCACACTCGATACAACTTTCTTTCGCCTGTTTC ACGATTTTGTTTATACTCTAATATTTCAGCACAATCTTTTTACTCTTTCAGCCTTTTTAAATTCAAGAATATGCAGAAGTTCAAAGT AATCAACATTAGCGATTTTCTTTTCTCTCCATGGTCTCACTTTTCCACTTTTTGTCTTGTCCACTAAAACCCTTGATTTTTCATCTGA ATAAATGCTACTATTAGGACACATAATATTAAAAGAAACCCCCATCTATTTAGTTATTTGTTTGGTCACTTATAACTTTAACAGAT GGGGTTTTTCTGTGCAACCAATTTTAAGGGTTTTCCAATACTTTAAAACACATACATACCAACACTTCAACGCACCTTTCAGCAA CTAAAATAAAAATGACGTTATTTCTATATGTATCAAGATAAGAAAGAACAAGTTCAAAACCATCAAAAAAAGACACCTTTTCAG GTGCTTTTTTTTATTTTATAAACTCATTCCCTGATCTCGACTTCGTTCTTTTTTTTACCTCTCGGTTATGAGTTAGTTCAAATTCGTTCT TTTTAGGTTCTAAATCGTGTTTTTCTTGGAATTGTGCTGTTTTATCCTTTACCTTGTCTACAAACCCCTTAAAAACGTTTTTAAAGG CTTTTAAGCGTCTGTACGTTCCTTAAGGAATTATTCCTTAGTGCTTTCTAGGTTAATGTCATGATAATAATGGTTTCTTAGACGTC AGGTGGCACTTTTCGGGGAAATGTCCGCGGAACCCCTATTTGTTTATTTTTCTAAATACATTCAAATATGTATCCGCTCATGAGA CAATAACCCTGATAAATGCTTCAATAATATTGAAAAAGGAAGAGTATGAGTATTCAACATTTCCGTGTCGCCCTTATTCCCTTTT TTGCGGCATTTTGCCTTCCTGTTTTTGCTCACCCAGAAACGCTGGTGAAAGTAAAAGATGCTGAAGATCAGTTGGGTGCACGA GTGGGTTACATCGAACTGGATCTCAACAGCGGTAAGATCCTTGAGAGTTTTCGCCCCGAAGAACGTTTTCCAATGATGAGCAC TTTTAAAGTTCTGCTATGTGGCGCGGTATTATCCCGTGTTGACGCCGGGCAAGAGCAACTCGGTCGCCGCATACACTATTCTCA GAATGACTTGGTTGAGTACTCACCAGTCACAGAAAAGCATCTTACGGATGGCATGACAGTAAGAGAATTATGCAGTGCTGCCA TAACCATGAGTGATAACACTGCGGCCAACTTACTTCTGACAACGATCGGAGGACCGAAGGAGCTAACCGCTTTTTTGCACAAC ATGGGGGATCATGTAACTCGCCTTGATCGTTGGGAACCGGAGCTGAATGAAGCCATACCAAACGACGAGCGTGACACCACGA TGCCTGCAGCAATGGCAACAACGTTGCGCAAACTATTAACTGGCGAACTACTTACTCTAGCTTCCCGGCAACAATTAATAGACT GGATGGAGGCGGATAAAGTTGCAGGACCACTTCTGCGCTCGGCCCTTCCGGCTGGCTGGTTTATTGCTGATAAATCTGGAGCC GGTGAGCGTGGGTCTCGCGGTATCATTGCAGCACTGGGGCCAGATGGTAAGCCCTCCCGTATCGTAGTTATCTACACGACGG GGAGTCAGGCAACTATGGATGAACGAAATAGACAGATCGCTGAGATAGGTGCCTCACTGATTAAGCATTGGTAACTGTCAGA CCAAGTTTACTCATATATACTTTAGATTGATTTAAAACTTCATTTTTAATTTAAAAGGATCTAGGTGAAGATCCTTTTTGATAATC TCATGACCAAAATCCCTTAACGTGAGTTTTCGTTCCACTGAGCGTCAGACCCCTTAATAAGATGATCTTCTTGAGATCGTTTTGG TCTGCGCGTAATCTCTTGCTCTGAAAACGAAAAAACCGCCTTGCAGGGAGGTTTTTCGAAGGTTCTCTGAGCTACCAACTCTTT GAACCGAGGTAACTGGCTTGCAGGAGCGCAGTCACCAAAACTTGTCCTTTCAGTTTAGCCTTAACCGGCGCATGACTTCAAGA CTAACTCCTCTAAATCAATTACCAGTGGCTGCTGCCAGTGGTGCTTTTGCATGTCTTTTCCGGGTTGGACTCAAGACGATAGTTAC CGGATAAGGCGCAGCGGTCGGACTGAACGGGGGGTTCGTGCATACAGTCCAGCTTGGAGCGAACTGCCTACCCGGAACTGA GTGTCAGGCGTGGAATGAGACAAACGCGGCCATAACAGCGGAATGACACCGGTAAACCGAAAGGCAGGAACAGGAGAGCG CACGAGGGAGCCGCCAGGGGGAAACGCCTGGTATCTTTATAGTCCTGTCGGGTTTCGCCACCACTGATTTGAGCGTCAGATTT CGTGATGCTTGTCAGGGGGCGGAGCCTATGGAAAAACGCTTTGCCCGAGCCTATGGAAAAACGCTTTGCCCCACACAGGCTCT TGAACAAGGGATAACAATTTGTCATAGCTGTATTCGTTACAAGGAGGATTTTCGAAAAAACACCCTAACGGGTGTTTTTTTATA GCTGGTCTCCCCAAGTGCTGTAAAAAACGCCGAATGAAGCGGATGAAATTGCTCATCGTCTCGTGAAAAAGCCGAAGAAGGT TAAGCCTGGGTATAAAAAGAAAATGAGCTATGAGATGGAGAAAGCCATAACCATGAGTGATAACACTGGTTTTAGAGCTAGA AATAGCAAGTTAAAATAAGGCTAGTCCGTTATCAACTTGAAAAAGTGGCACCGAGTCGGTGCTTTTTTTCTCGGTACCAAATTC CAGAAAAGAGGCCTCCCGAAAGGGGGGCCTTTTTTTCGTTTTGGTCCCTCAATGATCTCCTTATCCGGTTAAGATGGCAAGCTTG ACAAGTATTTCTTCAGAATTTACAATGAAGTTTACATGACGCTCTTAAAGGGGGTTTTAGATACCAGAGATGGATAAGAAATAC TCAATAGGCTTAGATATCGGCACAAATAGCGTCGGATGGGCGGTGATCACTGATGAATATAAGGTTCCGTCTAAAAAGTTCAA GGTTCTGGGAAATACAGACCGCCACAGTATCAAAAAAAATCTTATAGGGGCTCTTTTATTTGACAGTGGAGAGACAGCGGAA GCGACTCGTCTCAAACGGACAGCTCGTAGAAGGTATACACGTCGGAAGAATCGTATTTGTTATCTACAGGAGATTTTTTCAAAT GAGATGGCGAAAGTAGATGATAGTTTCTTTCATCGACTTGAAGAGTCTTTTTTTGGTGGAAGAAGACAAGAAGCATGAACGTCA TCCTATTTTTGGAAATATAGTAGATGAAGTTGCTTATCATGAGAAATATCCAACTATCTATCATCTGCGAAAAAAATTGGTAGAT TCTACTGATAAAGCGGATTTGCGCTTAATCTATTTGGCCTTAGCGCATATGATTAAGTTTCGTGGTCATTTTTTGATTGAGGGAG ATTTAAATCCTGATAATAGTGATGTGGACAAACTATTTATCCAGTTGGTACAAACCTACAATCAATTATTTGAAGAAAACCCTAT TAACGCAAGTGGAGTAGATGCTAAAGCGATTCTTTCTGCACGATTGAGTAAATCAAGACGATTAGAAAATCTCATTGCTCAGCT CCCCGGTGAGAAGAAAAATGGCTTATTTGGGAATCTCATTGCTTTGTCATTGGGTTTGACCCCTAATTTTAAATCAAATTTTGAT TTGGCAGAAGATGCTAAATTACAGCTTTCAAAAGATACTTACGATGATGATTTAGATAATTTATTGGCGCAAATTGGAGATCAA TATGCTGATTTGTTTTTGGCAGCTAAGAATTTATCAGATGCTATTTTACTTTCAGATATCCTAAGAGTAAATACTGAAATAACTA AGGCTCCCCTATCAGCTTCAATGATTAAACGCTACGATGAACATCATCAAGACTTGACTCTTTTAAAAGCTTTAGTTCGACAACA 
ACTTCCAGAAAAGTATAAAGAAATCTTTTTTGATCAATCAAAAAACGGATATGCAGGTTATATTGATGGGGGAGCTAGCCAAG AAGAATTTTATAAATTTATCAAACCAATTTTAGAAAAAATGGATGGTACTGAGGAATTATTGGTGAAACTAAATCGTGAAGATT TGCTGCGCAAGCAACGGACCTTTGACAACGGCTCTATTCCCCATCAAATTCACTTGGGTGAGCTGCATGCTATTTTGAGAAGAC AAGAAGACTTTTATCCATTTTTAAAAGACAATCGTGAGAAGATTGAAAAAATCTTGACTTTTCGAATTCCTTATTATGTTGGTCC ATTGGCGCGTGGCAATAGTCGTTTTGCATGGATGACTCGGAAGTCTGAAGAAACAATTACCCCATGGAATTTTGAAGAAGTTG TCGATAAAGGTGCTTCAGCTCAATCATTTATTGAACGCATGACAAACTTTGATAAAAATCTTCCAAATGAAAAAGTACTACCAA AACATAGTTTGCTTTATGAGTATTTTACGGTTTATAACGAATTGACAAAGGTCAAATATGTTACTGAAGGAATGCGAAAACCAG CATTTCTTTCAGGTGAACAGAAGAAAGCCATTGTTGATTTACTCTTCAAAACAAATCGAAAAGTAACCGTTAAGCAATTAAAAG AAGATTATTTCAAAAAAATAGAATGTTTTGATAGTGTTGAAATTTCAGGAGTTGAAGATAGATTTAATGCTTCATTAGGTACCT ACCATGATTTGCTAAAAATTATTAAAGATAAAGATTTTTTGGATAATGAAGAAAATGAAGATATCTTAGAGGATATTGTTTTAA CATTGACCTTATTTGAAGATAGGGAGATGATTGAGGAAAGACTTAAAACATATGCTCACCTCTTTGATGATAAGGTGATGAAA CAGCTTAAACGTCGCCGTTATACTGGTTGGGGACGTTTGTCTCGAAAATTGATTAATGGTATTAGGGATAAGCAATCTGGCAA AACAATATTAGATTTTTTGAAATCAGATGGTTTTGCCAATCGCAATTTTATGCAGCTGATCCATGATGATAGTTTGACATTTAAA GAAGACATTCAAAAAGCACAAGTGTCTGGACAAGGCGATAGTTTACATGAACATATTGCAAATTTAGCTGGTAGCCCTGCTAT TAAAAAAGGTATTTTACAGACTGTAAAAGTTGTTGATGAATTGGTCAAAGTAATGGGGCGGCATAAGCCAGAAAATATCGTTA TTGAAATGGCACGTGAAAATCAGACAACTCAAAAGGGCCAGAAAAATTCGCGAGAGCGTATGAAACGAATCGAAGAAGGTAT CAAAGAATTAGGAAGTCAGATTCTTAAAGAGCATCCTGTTGAAAATACTCAATTGCAAAATGAAAAGCTCTATCTCTATTATCT CCAAAATGGAAGAGACATGTATGTGGACCAAGAATTAGATATTAATCGTTTAAGTGATTATGATGTCGATCACATTGTTCCACA AAGTTTCCTTAAAGACGATTCAATAGACAATAAGGTCTTAACGCGTTCTGATAAAAATCGTGGTAAATCGGATAACGTTCCAAG TGAAGAAGTAGTCAAAAAGATGAAAAACTATTGGAGACAACTTCTAAACGCCAAGTTAATCACTCAACGTAAGTTTGATAATTT AACGAAAGCTGAACGTGGAGGTTTGAGTGAACTTGATAAAGCTGGTTTTATCAAACGCCAATTGGTTGAAACTCGCCAAATCA CTAAGCATGTGGCACAAATTTTGGATAGTCGCATGAATACTAAATACGATGAAAATGATAAACTTATTCGAGAGGTTAAAGTG ATTACCTTAAAATCTAAATTAGTTTCTGACTTCCGAAAAGATTTCCAATTCTATAAAGTACGTGAGATTAACAATTACCATCATG CCCATGATGCGTATCTAAATGCCGTCGTTGGAACTGCTTTGATTAAGAAATATCCAAAACTTGAATCGGAGTTTGTCTATGGTG ATTATAAAGTTTATGATGTTCGTAAAATGATTGCTAAGTCTGAGCAAGAAATAGGCAAAGCAACCGCAAAATATTTCTTTTACT CTAATATCATGAACTTCTTCAAAACAGAAATTACACTTGCAAATGGAGAGATTCGCAAACGCCCTCTAATCGAAACTAATGGGG AAACTGGAGAAATTGTCTGGGATAAAGGGCGAGATTTTGCCACAGTGCGCAAAGTATTGTCCATGCCCCAAGTCAATATTGTC AAGAAAACAGAAGTACAGACAGGCGGATTCTCCAAGGAGTCAATTTTACCAAAAAGAAATTCGGACAAGCTTATTGCTCGTAA AAAAGACTGGGATCCAAAAAAATATGGTGGTTTTGATAGTCCAACGGTAGCTTATTCAGTCCTAGTGGTTGCTAAGGTGGAAA AAGGGAAATCGAAGAAGTTAAAATCCGTTAAAGAGTTACTAGGGATCACAATTATGGAAAGAAGTTCCTTTGAAAAAAATCCG ATTGACTTTTTAGAAGCTAAAGGATATAAGGAAGTTAAAAAAGACTTAATCATTAAACTACCTAAATATAGTCTTTTTGAGTTA GAAAACGGTCGTAAACGGATGCTGGCTAGTGCCGGAGAATTACAAAAAGGAAATGAGCTGGCTCTGCCAAGCAAATATGTGA ATTTTTTATATTTAGCTAGTCATTATGAAAAGTTGAAGGGTAGTCCAGAAGATAACGAACAAAAACAATTGTTTGTGGAGCAGC ATAAGCATTATTTAGATGAGATTATTGAGCAAATCAGTGAATTTTCTAAGCGTGTTATTTTAGCAGATGCCAATTTAGATAAAG TTCTTAGTGCATATAACAAACATAGAGACAAACCAATACGTGAACAAGCAGAAAATATTATTCATTTATTTACGTTGACGAATC TTGGAGCTCCCGCTGCTTTTAAATATTTTGATACAACAATTGATCGTAAACGATATACGTCTACAAAAGAAGTTTTAGATGCCAC TCTTATCCATCAATCCATCACTGGTCTTTATGAAACACGCATTGATTTGAGTCAGCTAGGAGGTGACTGATAATCAAATGTCAG ACGAAAATGCCAATTATTGAAGCGGCTAACGCCGCTTTTTTTTGTTTCTGGTCTCCCTTGCTTGTACTTTACAGTATAGCTTCTAG AGATCTGCAGTGACTGGAGCTTCTAGAGATC

>pCas9g1-D15-cons

GATTAACTAATAAGGAGGGCAAACTACTAGAGATGAGTAAATCCTGGGGCAAATTCATCGAGGAGGAAGAGGCGGAAATGG CTTCCCGTCGTAATCTAATGATTGTCGATGGAACTAACTTAGGCTTTCGCTTCAAACATAACAATAGTAAAAAACCATTTGCCTC AAGTTATGTTTCAACTATTCAATCTCTGGCAAAATCCTACTCTGCCAGAACTACGATTGTTCTAGGTGATAAGGGCAAAAGCGT GTTCAGACTAGAACATCTACCAGAGTATAAAGGTAATCGTGATGAAAAGTACGCACAACGTACGGAAGAGGAGAAAGCGCTA GATGAGCAGTTCTTCGAATACCTCAAAGATGCTTTCGAGTTGTGTAAAACTACATTCCCAACTTTTACCATTCGTGGTGTAGAA GCAGATGACATGGCGGCGTATATTGTTAAGCTCATCGGGCATCTTTATGATCACGTTTGGCTAATATCTACAGATGGTGACTGG GATACTTTATTAACGGATAAAGTTTCTCGTTTTTCTTTCACAACACGTCGTGAGTATCATCTTCGTGATATGTATGAGCACCATA ACGTGGACGACGTAGAACAGTTTATCTCCCTGAAAGCAATTATGGGAGATCTAGGAGATAATATTCGTGGTGTTGAAGGAATA GGAGCAAAACGCGGATATAATATTATTCGTGAGTTTGGTAACGTACTGGATATTATTGATCAGCTTCCACTGCCTGGAAAGCA GAAATATATACAGAACCTGAATGCATCGGAAGAACTGCTTTTCCGAAACTTGATTCTGGTTGATTTACCTACCTACTGTGTGGA TGCTATTGCTGCTGTAGGTCAAGATGTGTTAGATAAGTTCACGAAAGACATCCTTGAGATCGCGGAACAATGATAAGCTCTTA AAGGGGGTTTTAGATACCAGAGATGGATAAGAAATACTCAATAGGCTTAGATATCGGCACAAATAGCGTCGGATGGGCGGTG 
ATCACTGATGAATATAAGGTTCCGTCTAAAAAGTTCAAGGTTCTGGGAAATACAGACCGCCACAGTATCAAAAAAAATCTTATA GGGGCTCTTTTATTTGACAGTGGAGAGACAGCGGAAGCGACTCGTCTCAAACGGACAGCTCGTAGAAGGTATACACGTCGGA AGAATCGTATTTGTTATCTACAGGAGATTTTTTCAAATGAGATGGCGAAAGTAGATGATAGTTTCTTTCATCGACTTGAAGAGT CTTTTTTGGTGGAAGAAGACAAGAAGCATGAACGTCATCCTATTTTTGGAAATATAGTAGATGAAGTTGCTTATCATGAGAAAT ATCCAACTATCTATCATCTGCGAAAAAAATTGGTAGATTCTACTGATAAAGCGGATTTGCGCTTAATCTATTTGGCCTTAGCGCA TATGATTAAGTTTCGTGGTCATTTTTTGATTGAGGGAGATTTAAATCCTGATAATAGTGATGTGGACAAACTATTTATCCAGTTG GTACAAACCTACAATCAATTATTTGAAGAAAACCCTATTAACGCAAGTGGAGTAGATGCTAAAGCGATTCTTTCTGCACGATTG AGTAAATCAAGACGATTAGAAAATCTCATTGCTCAGCTCCCCGGTGAGAAGAAAAATGGCTTATTTGGGAATCTCATTGCTTTG TCATTGGGTTTGACCCCTAATTTTAAATCAAATTTTTGATTTGGCAGAAGATGCTAAATTACAGCTTTCAAAAGATACTTACGATG ATGATTTAGATAATTTATTGGCGCAAATTGGAGATCAATATGCTGATTTGTTTTTGGCAGCTAAGAATTTATCAGATGCTATTTT ACTTTCAGATATCCTAAGAGTAAATACTGAAATAACTAAGGCTCCCCTATCAGCTTCAATGATTAAACGCTACGATGAACATCAT CAAGACTTGACTCTTTTAAAAGCTTTAGTTCGACAACAACTTCCAGAAAAGTATAAAGAAATCTTTTTTGATCAATCAAAAAACG GATATGCAGGTTATATTGATGGGGGAGCTAGCCAAGAAGAATTTTATAAATTTATCAAACCAATTTTAGAAAAAATGGATGGT ACTGAGGAATTATTGGTGAAACTAAATCGTGAAGATTTGCTGCGCAAGCAACGGACCTTTGACAACGGCTCTATTCCCCATCAA ATTCACTTGGGTGAGCTGCATGCTATTTTGAGAAGACAAGAAGACTTTTATCCATTTTTAAAAGACAATCGTGAGAAGATTGAA AAAATCTTGACTTTTTGAATTCCTTATTATGTTGGTCCATTGGCGCGTGGCAATAGTCGTTTTGCATGGATGACTCGGAAGTCTG AAGAAACAATTACCCCATGGAATTTTGAAGAAGTTGTCGATAAAGGTGCTTCAGCTCAATCATTTATTGAACGCATGACAAACT TTGATAAAAATCTTCCAAATGAAAAAGTACTACCAAAACATAGTTTGCTTTATGAGTATTTTACGGTTTATAACGAATTGACAAA GGTCAAATATGTTACTGAAGGAATGCGAAAACCAGCATTTCTTTCAGGTGAACAGAAGAAAGCCATTGTTGATTTACTCTTCAA AACAAATCGAAAAGTAACCGTTAAGCAATTAAAAGAAGATTATTTCAAAAAAATAGAATGTTTTGATAGTGTTGAAATTTCAG GAGTTGAAGATAGATTTAATGCTTCATTAGGTACCTACCATGATTTGCTAAAAATTATTAAAGATAAAGATTTTTTGGATAATG AAGAAAATGAAGATATCTTAGAGGATATTGTTTTAACATTGACCTTATTTGAAGATAGGGAGATGATTGAGGAAAGACTTAAA ACATATGCTCACCTCTTTGATGATAAGGTGATGAAACAGCTTAAACGTCGCCGTTATACTGGTTGGGGACGTTTGTCTCGAAAA TTGATTAATGGTATTAGGGATAAGCAATCTGGCAAAACAATATTAGATTTTTTGAAATCAGATGGTTTTGCCAATCGCAATTTTA TGCAGCTGATCCATGATGATAGTTTGACATTTAAAGAAGACATTCAAAAAGCACAAGTGTCTGGACAAGGCGATAGTTTACAT GAACATATTGCAAATTTAGCTGGTAGCCCTGCTATTAAAAAAGGTATTTTACAGACTGTAAAAGTTGTTGATGAATTGGTCAAA GTAATGGGGCGGCATAAGCCAGAAAATATCGTTATTGAAATGGCACGTGAAAATCAGACAACTCAAAAGGGCCAGAAAAATT CGCGAGAGCGTATGAAACGAATCGAAGAAGGTATCAAAGAATTAGGAAGTCAGATTCTTAAAGAGCATCCTGTTGAAAATAC TCAATTGCAAAATGAAAAGCTCTATCTCTATTATCTCCAAAATGGAAGAGACATGTATGTGGACCAAGAATTAGATATTAATCG TTTAAGTGATTATGATGTCGATCACATTGTTCCACAAAGTTTCCTTAAAGACGATTCAATAGACAATAAGGTCTTAACGCGTTCT GATAAAAATCGTGGTAAATCGGATAACGTTCCAAGTGAAGAAGTAGTCAAAAAGATGAAAAACTATTGGAGACAACTTCTAA ACGCCAAGTTAATCACTCAACGTAAGTTTGATAATTTAACGAAAGCTGAACGTGGAGGTTTGAGTGAACTTGATAAAGCTGGT TTTATCAAACGCCAATTGGTTGAAACTCGCCAAATCACTAAGCATGTGGCACAAATTTTGGATAGTCGCATGAATACTAAATAC GATGAAAATGATAAACTTATTCGAGAGGTTAAAGTGATTACCTTAAAATCTAAATTAGTTTCTGACTTCCGAAAAGATTTCCAA TTCTATAAAGTACGTGAGATTAACAATTACCATCATGCCCATGATGCGTATCTAAATGCCGTCGTTGGAACTGCTTTGATTAAG AAATATCCAAAACTTGAATCGGAGTTTGTCTATGGTGATTATAAAGTTTATGATGTTCGTAAAATGATTGCTAAGTCTGAGCAA GAAATAGGCAAAGCAACCGCAAAATATTTCTTTTACTCTAATATCATGAACTTCTTCAAAACAGAAATTACACTTGCAAATGGA GAGATTCGCAAACGCCCTCTAATCGAAACTAATGGGGAAACTGGAGAAATTGTCTGGGATAAAGGGCGAGATTTTGCCACAG TGCGCAAAGTATTGTCCATGCCCCAAGTCAATATTGTCAAGAAAACAGAAGTACAGACAGGCGGATTCTCCAAGGAGTCAATT TTACCAAAAAGAAATTCGGACAAGCTTATTGCTCGTAAAAAAGACTGGGATCCAAAAAAATATGGTGGTTTTGATAGTCCAAC GGTAGCTTATTCAGTCCTAGTGGTTGCTAAGGTGGAAAAAGGGAAATCGAAGAAGTTAAAATCCGTTAAAGAGTTACTAGGG ATCACAATTATGGAAAGAAGTTCCTTTGAAAAAAATCCGATTGACTTTTTAGAAGCTAAAGGATATAAGGAAGTTAAAAAAGA CTTAATCATTAAACTACCTAAATATAGTCTTTTTGAGTTAGAAAACGGTCGTAAACGGATGCTGGCTAGTGCCGGAGAATTACA AAAAGGAAATGAGCTGGCTCTGCCAAGCAAATATGTGAATTTTTTATATTTAGCTAGTCATTATGAAAAGTTGAAGGGTAGTCC AGAAGATAACGAACAAAAACAATTGTTTGTGGAGCAGCATAAGCATTATTTAGATGAGATTATTGAGCAAATCAGTGAATTTT CTAAGCGTGTTATTTTAGCAGATGCCAATTTAGATAAAGTTCTTAGTGCATATAACAAACATAGAGACAAACCAATACGTGAAC AAGCAGAAAATATTATTCATTTATTTACGTTGACGAATCTTGGAGCTCCCGCTGCTTTTAAATATTTTGATACAACAATTGATCG TAAACGATATACGTCTACAAAAGAAGTTTTAGATGCCACTCTTATCCATCAATCCATCACTGGTCTTTATGAAACACGCATTGAT TTGAGTCAGCTAGGAGGTGACTGATAATCAAATGTCAGACGAAAATGCCAATTATTGAAGCGGCTAACGCCGCTTTTTTTTGTT CTGGTCTCCCTTGCTTGTACTTTACAGTATAGCTTCTAGAGATCTGCAGTGACTGGAGCTTCTAGAGATCTGCAGTGACTGGGA AAACCCTGGCGACTAGTCTTGGACTCCTGTTGATAGATCCAGTAATGACCTCAGAACTCCATCTGGATTTGTTCAGAACGCTCG GTTGCCGCCGGGCGTTTTTTATTGGTGAGAATCCAGGGGTCCCCAATAATTACGATTTAAATCCTTCAAACTTCCCAAAGGCGA GCCCTAGTGACATTAGAAAACCGACTGTAAAAAGTACAGTCGGCATTATCTCATATTATAAAAGCCAGTCATTAGGCCTATCTG 
ACAATTCCTGAATAGAGTTCATAAACAATCCTGCATGATAACCATCACAAACAGAATGATGTACCTGTAAAGATAGCGGTAAAT ATATTGAATTACCTTTATTAATGAATTTTCCTGCTGTAATAATGGGTAGAAGGTAATTACTATTATTATTGATATTTAAGTTAAAC CCAGTAAATGAAGTCCATGGAATAATAGAAAGAGAAAAAGCATTTTCAGGTATAGGTGTTTTGGGAAACAATTTCCCCGAACC ATTATATTTCTCTACATCAGAAAGGTATAAATCATAAAACTCTTTGAAGTCATTCTTTACAGGAGTCCAAATACCAGAGAATGTT TTAGATACACCATCAAAAATTGTATAAAGTGGCTCTAACTTATCCCAATAACCTAACTCTCCGTCGCTATTGTAACCAGTTCTAA AAGCTGTATTTGAGTTTATCACCCTTGTCACTAAGAAAATAAATGCAGGGTAAAATTTATATCCTTCTTGTTTTATGTTTCGGTAT AAAACACTAATATCAATTTCTGTGGTTATACTAAAAGTCGTTTGTTGGTTCAAATAATGATTAAATATCTCTTTTCTCTTCCAATT GTCTAAATCAATTTTATTAAAGTTCATTTGATATGCCTCCTAAATTTTTATCTAAAGTGAATTTAGGAGGCTTACTTGTCTGCTTT CTTCATTAGAATCAATCCTTTTTTAAAAGTCAATCCCGTTTGTTGAAGACTTTTGTCCTTTTCCGCTGCATAAACAATATGGCCCG TTTGTTGAACTACTCTTTAATAAAATAATTTTTCCGTTCCCAATTCCACATTGCAATAATAGAAAATCCATCTTCATCGGCTTTTTC GTCATCATCTGTATGAATCAAATCGCCTTCTTCTGTGTCATCAAGGTTTAATTTTTTATGTATTTCTTTTAACAAACCACCATAGG AGATTAACCTTTTACGGTGTAAACCTTCCTCCAAATCAGACAAACGTTTCAAATTCTTTTCTTCATCATCGGTCATAAAATCCGTA TCCTTTACAGGATATTTTGCAGTTTCGTCAATTGCCGATTGTATATCCGATTTATATTTATTTTTCGGTCGAATCATTTGAACTTTT ACATTTGGATCATAGTCTAATTTCATTGCCTTTTTCCAAAATTGAATCCATTGTTTTTGATTCACGTAGTTTTCTGTATTCTTAAAA TAAGTTGGTTCCACACATACCAATACATGCATGTGCTGATTATAAGAATTATCTTTATTATTTATTGTCACTTCCGTTGCACGCAT AAAACCAACAAGATTTTTATTAATTTTTTTATATTGCATCATTCGGCGAAATCCTTGAGCCATATCTGACAAACTCTTATTTAATT CTTCGCCATCATAAACATTTTTAACTGTTAATGTGAGAAACAACCAACGAACTGTTGGCTTTTGTTTAATAACTTCAGCAACAAC CTTTTGTGACTGAATGCCATGTTTCATTGCTCTCCTCCAGTTGCACATTGGACAAAGCCTGGATTTACAAAACCACACTCGATAC AACTTTCTTTCGCCTGTTTCACGATTTTGTTTATACTCTAATATTTCAGCACAATCTTTTACTCTTTCAGCCTTTTTAAATTCAAGAA TATGCAGAAGTTCAAAGTAATCAACATTAGCGATTTTCTTTTCTCTCCATGGTCTCACTTTTCCACTTTTTGTCTTGTCCACTAAAA CCCTTGATTTTTCATCTGAATAAATGCTACTATTAGGACACATAATATTAAAAGAAACCCCCATCTATTTAGTTATTTGTTTGGTC ACTTATAACTTTAACAGATGGGGTTTTTCTGTGCAACCAATTTTAAGGGTTTTCCAATACTTTAAAACACATACATACCAACACTT CAACGCACCTTTCAGCAACTAAAATAAAAATGACGTTATTTCTATATGTATCAAGATAAGAAAGAACAAGTTCAAAACCATCAA AAAAAGACACCTTTTCAGGTGCTTTTTTTATTTTATAAACTCATTCCCTGATCTCGACTTCGTTCTTTTTTTACCTCTCGGTTATGA GTTAGTTCAAATTCGTTCTTTTTAGGTTCTAAATCGTGTTTTTCTTGGAATTGTGCTGTTTTATCCTTTACCTTGTCTACAAACCCC TTAAAAACGTTTTTAAAGGCTTTTAAGCGTCTGTACGTTCCTTAAGGAATTATTCCTTAGTGCTTTCTAGGTTAATGTCATGATA ATAATGGTTTCTTAGACGTCAGGTGGCACTTTTCGGGGAAATGTCCGCGGAACCCCTATTTGTTTATTTTTCTAAATACATTCAA ATATGTATCCGCTCATGAGACAATAACCCTGATAAATGCTTCAATAATATTGAAAAAGGAAGAGTATGAGTATTCAACATTTCC GTGTCGCCCTTATTCCCTTTTTTGCGGCATTTTGCCTTCCTGTTTTTTGCTCACCCAGAAACGCTGGTGAAAGTAAAAGATGCTGA AGATCAGTTGGGTGCACGAGTGGGTTACATCGAACTGGATCTCAACAGCGGTAAGATCCTTGAGAGTTTTCGCCCCGAAGAAC GTTTTCCAATGATGAGCACTTTTAAAGTTCTGCTATGTGGCGCGGTATTATCCCGTGTTGACGCCGGGCAAGAGCAACTCGGTC GCCGCATACACTATTCTCAGAATGACTTGGTTGAGTACTCACCAGTCACAGAAAAGCATCTTACGGATGGCATGACAGTAAGA GAATTATGCAGTGCTGCCATAACCATGAGTGATAACACTGCGGCCAACTTACTTCTGACAACGATCGGAGGACCGAAGGAGCT AACCGCTTTTTTGCACAACATGGGGGATCATGTAACTCGCCTTGATCGTTGGGAACCGGAGCTGAATGAAGCCATACCAAACG ACGAGCGTGACACCACGATGCCTGCAGCAATGGCAACAACGTTGCGCAAACTATTAACTGGCGAACTACTTACTCTAGCTTCCC GGCAACAATTAATAGACTGGATGGAGGCGGATAAAGTTGCAGGACCACTTCTGCGCTCGGCCCTTCCGGCTGGCTGGTTTATT GCTGATAAATCTGGAGCCGGTGAGCGTGGGTCTCGCGGTATCATTGCAGCACTGGGGCCAGATGGTAAGCCCTCCCGTATCG TAGTTATCTACACGACGGGGAGTCAGGCAACTATGGATGAACGAAATAGACAGATCGCTGAGATAGGTGCCTCACTGATTAA GCATTGGTAACTGTCAGACCAAGTTTACTCATATATACTTTAGATTGATTTAAAACTTCATTTTTAATTTAAAAGGATCTAGGTG AAGATCCTTTTTGATAATCTCATGACCAAAATCCCTTAACGTGAGTTTTCGTTCCACTGAGCGTCAGACCCCTTAATAAGATGAT CTTCTTGAGATCGTTTTGGTCTGCGCGTAATCTCTTGCTCTGAAAACGAAAAAACCGCCTTGCAGGGAGGTTTTTCGAAGGTTC TCTGAGCTACCAACTCTTTGAACCGAGGTAACTGGCTTGCAGGAGCGCAGTCACCAAAACTTGTCCTTTCAGTTTAGCCTTAAC CGGCGCATGACTTCAAGACTAACTCCTCTAAATCAATTACCAGTGGCTGCTGCCAGTGGTGCTTTTGCATGTCTTTCCGGGTTG GACTCAAGACGATAGTTACCGGATAAGGCGCAGCGGTCGGACTGAACGGGGGGTTCGTGCATACAGTCCAGCTTGGAGCGA ACTGCCTACCCGGAACTGAGTGTCAGGCGTGGAATGAGACAAACGCGGCCATAACAGCGGAATGACACCGGTAAACCGAAA GGCAGGAACAGGAGAGCGCACGAGGGAGCCGCCAGGGGGAAACGCCTGGTATCTTTATAGTCCTGTCGGGTTTCGCCACCAC TGATTTGAGCGTCAGATTTCGTGATGCTTGTCAGGGGGCGGAGCCTATGGAAAAACGCTTTGCCCGAGCCTATGGAAAAACGC TTTGCCCCACACAGGCTCTTGAACAAGGGATAACAATTTGTCATAGCTGTATTCGTTACAAGGAGGATTTTCGAAAAAACACCC TAACGGGTGTTTTTTTATAGCTGGTCTCCCCAAGTGCTGTAAAAAACGCCGAATGAAGCGGATGAAATTGCTCATCGTCTCGTG AAAAAGCCGAAGAAGGTTAAGCCTGGGTATAAAAAGAAAATGAGCTATGAGATGGAGAAAGCCATAACCATGAGTGATAAC ACTGGTTTTAGAGCTAGAAATAGCAAGTTAAAATAAGGCTAGTCCGTTATCAACTTGAAAAAGTGGCACCGAGTCGGTGCTTT TTTTCTCGGTACCAAATTCCAGAAAAGAGGCCTCCCGAAAGGGGGGCCTTTTTTCGTTTTGGTCCCTCAATGATCTCCTTATCCG GTTAAGATGGCAAGCTTGACAAGTATTTCTTCAGAATTTACAATGAAGTTTACATGAC 
$>$ pCas9g2-D15-cons

GATTAACTAATAAGGAGGGCAAACTACTAGAGATGAGTAAATCCTGGGGCAAATTCATCGAGGAGGAAGAGGCGGAAATGG CTTCCCGTCGTAATCTAATGATTGTCGATGGAACTAACTTAGGCTTTCGCTTCAAACATAACAATAGTAAAAAACCATTTGCCTC AAGTTATGTTTCAACTATTCAATCTCTGGCAAAATCCTACTCTGCCAGAACTACGATTGTTCTAGGTGATAAGGGCAAAAGCGT GTTCAGACTAGAACATCTACCAGAGTATAAAGGTAATCGTGATGAAAAGTACGCACAACGTACGGAAGAGGAGAAAGCGCTA GATGAGCAGTTCTTCGAATACCTCAAAGATGCTTTCGAGTTGTGTAAAACTACATTCCCAACTTTTACCATTCGTGGTGTAGAA GCAGATGACATGGCGGCGTATATTGTTAAGCTCATCGGGCATCTTTATGATCACGTTTGGCTAATATCTACAGATGGTGACTGG GATACTTTATTAACGGATAAAGTTTCTCGTTTTTCTTTCACAACACGTCGTGAGTATCATCTTCGTGATATGTATGAGCACCATA ACGTGGACGACGTAGAACAGTTTATCTCCCTGAAAGCAATTATGGGAGATCTAGGAGATAATATTCGTGGTGTTGAAGGAATA GGAGCAAAACGCGGATATAATATTATTCGTGAGTTTGGTAACGTACTGGATATTATTGATCAGCTTCCACTGCCTGGAAAGCA GAAATATATACAGAACCTGAATGCATCGGAAGAACTGCTTTTCCGAAACTTGATTCTGGTTGATTTACCTACCTACTGTGTGGA TGCTATTGCTGCTGTAGGTCAAGATGTGTTAGATAAGTTCACGAAAGACATCCTTGAGATCGCGGAACAATGATAAGCTCTTA AAGGGGGTTTTAGATACCAGAGATGGATAAGAAATACTCAATAGGCTTAGATATCGGCACAAATAGCGTCGGATGGGCGGTG ATCACTGATGAATATAAGGTTCCGTCTAAAAAGTTCAAGGTTCTGGGAAATACAGACCGCCACAGTATCAAAAAAAATCTTATA GGGGCTCTTTTATTTGACAGTGGAGAGACAGCGGAAGCGACTCGTCTCAAACGGACAGCTCGTAGAAGGTATACACGTCGGA AGAATCGTATTTGTTATCTACAGGAGATTTTTTCAAATGAGATGGCGAAAGTAGATGATAGTTTCTTTCATCGACTTGAAGAGT CTTTTTTGGTGGAAGAAGACAAGAAGCATGAACGTCATCCTATTTTTGGAAATATAGTAGATGAAGTTGCTTATCATGAGAAAT ATCCAACTATCTATCATCTGCGAAAAAAATTGGTAGATTCTACTGATAAAGCGGATTTGCGCTTAATCTATTTGGCCTTAGCGCA TATGATTAAGTTTCGTGGTCATTTTTTGATTGAGGGAGATTTAAATCCTGATAATAGTGATGTGGACAAACTATTTATCCAGTTG GTACAAACCTACAATCAATTATTTGAAGAAAACCCTATTAACGCAAGTGGAGTAGATGCTAAAGCGATTCTTTCTGCACGATTG AGTAAATCAAGACGATTAGAAAATCTCATTGCTCAGCTCCCCGGTGAGAAGAAAAATGGCTTATTTGGGAATCTCATTGCTTTG TCATTGGGTTTGACCCCTAATTTTAAATCAAATTTTGATTTGGCAGAAGATGCTAAATTACAGCTTTCAAAAGATACTTACGATG ATGATTTAGATAATTTATTGGCGCAAATTGGAGATCAATATGCTGATTTGTTTTTGGCAGCTAAGAATTTATCAGATGCTATTTT ACTTTCAGATATCCTAAGAGTAAATACTGAAATAACTAAGGCTCCCCTATCAGCTTCAATGATTAAACGCTACGATGAACATCAT CAAGACTTGACTCTTTTAAAAGCTTTAGTTCGACAACAACTTCCAGAAAAGTATAAAGAAATCTTTTTTGATCAATCAAAAAACG GATATGCAGGTTATATTGATGGGGGAGCTAGCCAAGAAGAATTTTATAAATTTATCAAACCAATTTTAGAAAAAATGGATGGT ACTGAGGAATTATTGGTGAAACTAAATCGTGAAGATTTGCTGCGCAAGCAACGGACCTTTGACAACGGCTCTATTCCCCATCAA ATTCACTTGGGTGAGCTGCATGCTATTTTGAGAAGACAAGAAGACTTTTATCCATTTTTAAAAGACAATCGTGAGAAGATTGAA AAAATCTTGACTTTTCGAATTCCTTATTATGTTGGTCCATTGGCGCGTGGCAATAGTCGTTTTGCATGGATGACTCGGAAGTCTG AAGAAACAATTACCCCATGGAATTTTGAAGAAGTTGTCGATAAAGGTGCTTCAGCTCAATCATTTATTGAACGCATGACAAACT TTGATAAAAATCTTCCAAATGAAAAAGTACTACCAAAACATAGTTTGCTTTATGAGTATTTTACGGTTTATAACGAATTGACAAA GGTCAAATATGTTACTGAAGGAATGCGAAAACCAGCATTTCTTTCAGGTGAACAGAAGAAAGCCATTGTTGATTTACTCTTCAA AACAAATCGAAAAGTAACCGTTAAGCAATTAAAAGAAGATTATTTTAAAAAAATAGAATGTTTTGATAGTGTTGAAATTTCAG GAGTTGAAGATAGATTTAATGCTTCATTAGGTACCTACCATGATTTGCTAAAAATTATTAAAGATAAAGATTTTTTGGATAATG AAGAAAATGAAGATATCTTAGAGGATATTGTTTTAACATTGACCTTATTTGAAGATAGGGAGATGATTGAGGAAAGACTTAAA ACATATGCTCACCTCTTTGATGATAAGGTGATGAAACAGCTTAAACGTCGCCGTTATACTGGTTGGGGACGTTTGTCTCGAAAA TTGATTAATGGTATTAGGGATAAGCAATCTGGCAAAACAATATTAGATTTTTTTGAAATCAGATGGTTTTGCCAATCGCAATTTTA TGCAGCTGATCCATGATGATAGTTTGACATTTAAAGAAGACATTCAAAAAGCACAAGTGTCTGGACAAGGCGATAGTTTACAT GAACATATTGCAAATTTAGCTGGTAGCCCTGCTATTAAAAAAGGTATTTTACAGACTGTAAAAGTTGTTGATGAATTGGTCAAA GTAATGGGGCGGCATAAGCCAGAAAATATCGTTATTGAAATGGCACGTGAAAATCAGACAACTCAAAAGGGCCAGAAAAATT CGCGAGAGCGTATGAAACGAATCGAAGAAGGTATCAAAGAATTAGGAAGTCAGATTCTTAAAGAGCATCCTGTTGAAAATAC TCAATTGCAAAATGAAAAGCTCTATCTCTATTATCTCCAAAATGGAAGAGACATGTATGTGGACCAAGAATTAGATATTAATCG TTTAAGTGATTATGATGTCGATCACATTGTTCCACAAAGTTTCCTTAAAGACGATTCAATAGACAATAAGGTCTTAACGCGTTCT GATAAAAATCGTGGTAAATCGGATAACGTTCCAAGTGAAGAAGTAGTCAAAAAGATGAAAAACTATTGGAGACAACTTCTAA ACGCCAAGTTAATCACTCAACGTAAGTTTGATAATTTAACGAAAGCTGAACGTGGAGGTTTGAGTGAACTTGATAAAGCTGGT TTTATCAAACGCCAATTGGTTGAAACTCGCCAAATCACTAAGCATGTGGCACAAATTTTGGATAGTCGCATGAATACTAAATAC GATGAAAATGATAAACTTATTCGAGAGGTTAAAGTGATTACCTTAAAATCTAAATTAGTTTCTGACTTCCGAAAAGATTTCCAA TTCTATAAAGTACGTGAGATTAACAATTACCATCATGCCCATGATGCGTATCTAAATGCCGTCGTTGGAACTGCTTTGATTAAG AAATATCCAAAACTTGAATCGGAGTTTGTCTATGGTGATTATAAAGTTTATGATGTTCGTAAAATGATTGCTAAGTCTGAGCAA GAAATAGGCAAAGCAACCGCAAAATATTTCTTTTACTCTAATATCATGAACTTCTTCAAAACAGAAATTACACTTGCAAATGGA GAGATTCGCAAACGCCCTCTAATCGAAACTAATGGGGAAACTGGAGAAATTGTCTGGGATAAAGGGCGAGATTTTGCCACAG TGCGCAAAGTATTGTCCATGCCCCAAGTCAATATTGTCAAGAAAACAGAAGTACAGACAGGCGGATTCTCCAAGGAGTCAATT 
TTACCAAAAAGAAATTCGGACAAGCTTATTGCTCGTAAAAAAGACTGGGATCCAAAAAAATATGGTGGTTTTGATAGTCCAAC GGTAGCTTATTCAGTCCTAGTGGTTGCTAAGGTGGAAAAAGGGAAATCGAAGAAGTTAAAATCCGTTAAAGAGTTACTAGGG ATCACAATTATGGAAAGAAGTTCCTTTGAAAAAAATCCGATTGACTTTTTAGAAGCTAAAGgATATAAGGAAGTTAAAAAAGA CTTAATCATTAAACTACCTAAATATAGTCTTTTTGAGTTAGAAAACGGTCGTAAACGGATGCTGGCTAGTGCCGGAGAATTACA AAAAGGAAATGAGCTGGCTCTGCCAAGCAAATATGTGAATTTTTTATATTTAGCTAGTCATTATGAAAAGTTGAAGGGTAGTCC AGAAGATAACGAACAAAAACAATTGTTTGTGGAGCAGCATAAGCATTATTTAGATGAGATTATTGAGCAAATCAGTGAATTTT CTAAGCGTGTTATTTTAGCAGATGCCAATTTAGATAAAGTTCTTAGTGCATATAACAAACATAGAGACAAACCAATACGTGAAC AAGCAGAAAATATTATTCATTTATTTACGTTGACGAATCTTGGAGCTCCCGCTGCTTTTAAATATTTTGATACAACAATTGATCG TAAACGATATACGTCTACAAAAGAAGTTTTAGATGCCACTCTTATCCATCAATCCATCACTGGTCTTTATGAAACACGCATTGAT TTGAGTCAGCTAGGAGGTGACTGATAATCAAATGTCAGACGAAAATGCCAATTATTGAAGCGGCTAACGCCGCTTTTTTTGTTT CTGGTCTCCCTTGCTTGTACTTTACAGTATAGCTTCTAGAGATCTGCAGTGACTGGAGCTTCTAGAGATCAGGTCAGACGCGAT TTCCTGGGTGCTCGAGCCATGGGAAAGCGGTGGTGGAAAAAACGCCGAATGAAGCGGATGAAATTGCTCATCGTCTCGTGAA AAAGCCGAAGAAGGTTAAGCCTGGGTATAAAAAGAAAATGAGCTATGAGATGGAGAAATTGAAGTCATGCGCCGGTTAGTTT TAGAGCTAGAAATAGCAAGTTAAAATAAGGCTAGTCCGTTATCAACTTGAAAAAGTGGCACCGAGTCGGTGCTTTTTTTCTCG GTACCAAATTCCAGAAAAGAGGCCTCCCGAAAGGGGGGCCTTTTTTCGTTTTGGTCCCTCAATGATCCGACAGGAAGTGGTAT TAGCGACTCCTACAAGGAATAGGTGTCTGGGTCATGCAGTGACTGGGAAAACCCTGGCGACTAGTCTTGGACTCCTGTTGATA GATCCAGTAATGACCTCAGAACTCCATCTGGATTTGTTCAGAACGCTCGGTTGCCGCCGGGCGTTTTTTATTGGTGAGAATCCA GGGGTCCCCAATAATTACGATTTAAATCCTTCAAACTTCCCAAAGGCGAGCCCTAGTGACATTAGAAAACCGACTGTAAAAAGT ACAGTCGGCATTATCTCATATTATAAAAGCCAGTCATTAGGCCTATCTGACAATTCCTGAATAGAGTTCATAAACAATCCTGCAT GATAACCATCACAAACAGAATGATGTACCTGTAAAGATAGCGGTAAATATATTGAATTACCTTTATTAATGAATTTTCCTGCTGT AATAATGGGTAGAAGGTAATTACTATTATTATTGATATTTAAGTTAAACCCAGTAAATGAAGTCCATGGAATAATAGAAAGAG AAAAAGCATTTTCAGGTATAGGTGTTTTGGGAAACAATTTCCCCGAACCATTATATTTCTCTACATCAGAAAGGTATAAATCATA AAACTCTTTGAAGTCATTCTTTACAGGAGTCCAAATACCAGAGAATGTTTTAGATACACCATCAAAAATTGTATAAAGTGGCTC TAACTTATCCCAATAACCTAACTCTCCGTCGCTATTGTAACCAGTTCTAAAAGCTGTATTTGAGTTTATCACCCTTGTCACTAAGA AAATAAATGCAGGGTAAAATTTATATCCTTCTTGTTTTATGTTTCGGTATAAAACACTAATATCAATTTCTGTGGTTATACTAAAA GTCGTTTGTTGGTTCAAATAATGATTAAATATCTCTTTTCTCTTCCAATTGTCTAAATCAATTTTATTAAAGTTCATTTGATATGCC TCCTAAATTTTTATCTAAAGTGAATTTAGGAGGCTTACTTGTCTGCTTTCTTCATTAGAATCAATCCTTTTTTAAAAGTCAATCCC GTTTGTTGAAGACTTTTGTCCTTTTCCGCTGCATAAACAATATGGCCCGTTTGTTGAACTACTCTTTAATAAAATAATTTTTCCGT TCCCAATTCCACATTGCAATAATAGAAAATCCATCTTCATCGGCTTTTTCGTCATCATCTGTATGAATCAAATCGCCTTCTTCTGT GTCATCAAGGTTTAATTTTTTATGTATTTCTTTTAACAAACCACCATAGGAGATTAACCTTTTACGGTGTAAACCTTCCTCCAAAT CAGACAAACGTTTCAAATTCTTTTCTTCATCATCGGTCATAAAATCCGTATCCTTTACAGGATATTTTGCAGTTTCGTCAATTGCC GATTGTATATCCGATTTATATTTATTTTTCGGTCGAATCATTTGAACTTTTACATTTGGATCATAGTCTAATTTCATTGCCTTTTTC CAAAATTGAATCCATTGTTTTTGATTCACGTAGTTTTCTGTATTCTTAAAATAAGTTGGTTCCACACATACCAATACATGCATGTG CTGATTATAAGAATTATCTTTATTATTTATTGTCACTTCCGTTGCACGCATAAAACCAACAAGATTTTTATTAATTTTTTTATATTG CATCATTCGGCGAAATCCTTGAGCCATATCTGACAAACTCTTATTTAATTCTTCGCCATCATAAACATTTTTAACTGTTAATGTGA GAAACAACCAACGAACTGTTGGCTTTTGTTTAATAACTTCAGCAACAACCTTTTGTGACTGAATGCCATGTTTCATTGCTCTCCT CCAGTTGCACATTGGACAAAGCCTGGATTTACAAAACCACACTCGATACAACTTTCTTTCGCCTGTTTCACGATTTTGTTTATACT CTAATATTTCAGCACAATCTTTTACTCTTTCAGCCTTTTTAAATTCAAGAATATGCAGAAGTTCAAAGTAATCAACATTAGCGATT TTCTTTTCTCTCCATGGTCTCACTTTTCCACTTTTTGTCTTGTCCACTAAAACCCTTGATTTTTCATCTGAATAAATGCTACTATTAG GACACATAATATTAAAAGAAACCCCCATCTATTTAGTTATTTGTTTGGTCACTTATAACTTTAACAGATGGGGTTTTTCTGTGCA ACCAATTTTAAGGGTTTTCCAATACTTTAAAACACATACATACCAACACTTCAACGCACCTTTCAGCAACTAAAATAAAAATGAC GTTATTTCTATATGTATCAAGATAAGAAAGAACAAGTTCAAAACCATCAAAAAAAGACACCTTTTCAGGTGCTTTTTTTATTTTA TAAACTCATTCCCTGATCTCGACTTCGTTCTTTTTTTACCTCTCGGTTATGAGTTAGTTCAAATTCGTTCTTTTTAGGTTCTAAATC GTGTTTTTCTTGGAATTGTGCTGTTTTATCCTTTACCTTGTCTACAAACCCCTTAAAAACGTTTTTAAAGGCTTTTAAGCGTCTGT ACGTTCCTTAAGGAATTATTCCTTAGTGCTTTCTAGGTTAATGTCATGATAATAATGGTTTCTTAGACGTCAGGTGGCACTTTTC GGGGAAATGTCCGCGGAACCCCTATTTGTTTATTTTTCTAAATACATTCAAATATGTATCCGCTCATGAGACAATAACCCTGATA AATGCTTCAATAATATTGAAAAAGGAAGAGTATGAGTATTCAACATTTCCGTGTCGCCCTTATTCCCTTTTTTGCGGCATTTTGC CTTCCTGTTTTTGCTCACCCAGAAACGCTGGTGAAAGTAAAAGATGCTGAAGATCAGTTGGGTGCACGAGTGGGTTACATCGA ACTGGATCTCAACAGCGGTAAGATCCTTGAGAGTTTTCGCCCCGAAGAACGTTTTCCAATGATGAGCACTTTTAAAGTTCTGCT ATGTGGCGCGGTATTATCCCGTGTTGACGCCGGGCAAGAGCAACTCGGTCGCCGCATACACTATTCTCAGAATGACTTGGTTG AGTACTCACCAGTCACAGAAAAGCATCTTACGGATGGCATGACAGTAAGAGAATTATGCAGTGCTGCCATAACCATGAGTGAT AACACTGCGGCCAACTTACTTCTGACAACGATCGGAGGACCGAAGGAGCTAACCGCTTTTTTGCACAACATGGGGGATCATGT AACTCGCCTTGATCGTTGGGAACCGGAGCTGAATGAAGCCATACCAAACGACGAGCGTGACACCACGATGCCTGCAGCAATG 
GCAACAACGTTGCGCAAACTATTAACTGGCGAACTACTTACTCTAGCTTCCCGGCAACAATTAATAGACTGGATGGAGGCGGA TAAAGTTGCAGGACCACTTCTGCGCTCGGCCCTTCCGGCTGGCTGGTTTATTGCTGATAAATCTGGAGCCGGTGAGCGTGGGT CTCGCGGTATCATTGCAGCACTGGGGCCAGATGGTAAGCCCTCCCGTATCGTAGTTATCTACACGACGGGGAGTCAGGCAACT ATGGATGAACGAAATAGACAGATCGCTGAGATAGGTGCCTCACTGATTAAGCATTGGTAACTGTCAGACCAAGTTTACTCATA TATACTTTAGATTGATTTAAAACTTCATTTTTAATTTAAAAGGATCTAGGTGAAGATCCTTTTTGATAATCTCATGACCAAAATCC CTTAACGTGAGTTTTCGTTCCACTGAGCGTCAGACCCCTTAATAAGATGATCTTCTTGAGATCGTTTTGGTCTGCGCGTAATCTC TTGCTCTGAAAACGAAAAAACCGCCTTGCAGGGAGGTTTTTTCGAAGGTTCTCTGAGCTACCAACTCTTTGAACCGAGGTAACTG GCTTGCAGGAGCGCAGTCACCAAAACTTGTCCTTTCAGTTTAGCCTTAACCGGCGCATGACTTCAAGACTAACTCCTCTAAATC AATTACCAGTGGCTGCTGCCAGTGGTGCTTTTGCATGTCTTTCCGGGTTGGACTCAAGACGATAGTTACCGGATAAGGCGCAG CGGTCGGACTGAACGGGGGGTTCGTGCATACAGTCCAGCTTGGAGCGAACTGCCTACCCGGAACTGAGTGTCAGGCGTGGAA TGAGACAAACGCGGCCATAACAGCGGAATGACACCGGTAAACCGAAAGGCAGGAACAGGAGAGCGCACGAGGGAGCCGCC AGGGGGAAACGCCTGGTATCTTTATAGTCCTGTCGGGTTTCGCCACCACTGATTTGAGCGTCAGATTTCGTGATGCTTGTCAGG GGGCGGAGCCTATGGAAAAACGCTTTGCCCGAGCCTATGGAAAAACGCTTTGCCCCACACAGGCTCTTGAACAAGGGATAAC AATTTGTCATAGCTGTATTCGTTACAAGGAGGATTTTTGAAAAAACACCCTAACGGGTGTTTTTTTATAGCTGGTCTCCCCAAGT GCTGTAAAAAACGCCGAATGAAGCGGATGAAATTGCTCATCGTCTCGTGAAAAAGCCGAAGAAGGTTAAGCCTGGGTATAAA AAGAAAATGAGCTATGAGATGGAGAAAGCCATAACCATGAGTGATAACACTGGTTTTAGAGCTAGAAATAGCAAGTTAAAAT AAGGCTAGTCCGTTATCAACTTGAAAAAGTGGCACCGAGTCGGTGCTTTTTTTTCTCGGTACCAAATTCCAGAAAAGAGGCCTCC CGAAAGGGGGGCCTTTTTTCGTTTTGGTCCCTCAATGATCTCCTTATCCGGTTAAGATGGCAAGCTTGACAAGTATTTCTTCAGA ATTTACAATGAAGTTTACATGAC

$>$ pCas9g1

tccttatccggttaagatggCTTTTAAATCAATTTTCAGCTCCTGTATACAATTACCAAAGTTTTTCTGAATGAAGCCATGTGTTTTGACA CATTCTATACTCACAAGGAGGTGAGACACatggataagaaatactcaataggctTAGATATCGGCACAAATAGCGTCGGATGGGCGGT GATCACTGATGAATATAAGGTTCCGTCTAAAAAGTTCAAGGTTCTGGGAAATACAGACCGCCACAGTATCAAAAAAAATCTTAT AGGGGCTCTTTTATTTGACAGTGGAGAGACAGCGGAAGCGACTCGTCTCAAACGGACAGCTCGTAGAAGGTATACACGTCGG AAGAATCGTATTTGTTATCTACAGGAGATTTTTTCAAATGAGATGGCGAAAGTAGATGATAGTTTCTTTCATCGACTTGAAGAG TCTTTTTTGGTGGAAGAAGACAAGAAGCATGAACGTCATCCTATTTTTGGAAATATAGTAGATGAAGTTGCTTATCATGAGAAA TATCCAACTATCTATCATCTGCGAAAAAAATTGGTAGATTCTACTGATAAAGCGGATTTGCGCTTAATCTATTTGGCCTTAGCGC ATATGATTAAGTTTCGTGGTCATTTTTTGATTGAGGGAGATTTAAATCCTGATAATAGTGATGTGGACAAACTATTTATCCAGTT GGTACAAACCTACAATCAATTATTTGAAGAAAACCCTATTAACGCAAGTGGAGTAGATGCTAAAGCGATTCTTTCTGCACGATT GAGTAAATCAAGACGATTAGAAAATCTCATTGCTCAGCTCCCCGGTGAGAAGAAAAATGGCTTATTTGGGAATCTCATTGCTTT GTCATTGGGTTTGACCCCTAATTTTAAATCAAATTTTGATTTGGCAGAAGATGCTAAATTACAGCTTTCAAAAGATACTTACGAT GATGATTTAGATAATTTATTGGCGCAAATTGGAGATCAATATGCTGATTTGTTTTTGGCAGCTAAGAATTTATCAGATGCTATTT TACTTTCAGATATCCTAAGAGTAAATACTGAAATAACTAAGGCTCCCCTATCAGCTTCAATGATTAAACGCTACGATGAACATCA TCAAGACTTGACTCTTTTAAAAGCTTTAGTTCGACAACAACTTCCAGAAAAGTATAAAGAAATCTTTTTTTGATCAATCAAAAAAC GGATATGCAGGTTATATTGATGGGGGAGCTAGCCAAGAAGAATTTTATAAATTTATCAAACCAATTTTAGAAAAAATGGATGG TACTGAGGAATTATTGGTGAAACTAAATCGTGAAGATTTGCTGCGCAAGCAACGGACCTTTGACAACGGCTCTATTCCCCATCA AATTCACTTGGGTGAGCTGCATGCTATTTTGAGAAGACAAGAAGACTTTTATCCATTTTTAAAAGACAATCGTGAGAAGATTGA AAAAATCTTGACTTTTCGAATTCCTTATTATGTTGGTCCATTGGCGCGTGGCAATAGTCGTTTTGCATGGATGACTCGGAAGTCT GAAGAAACAATTACCCCATGGAATTTTGAAGAAGTTGTCGATAAAGGTGCTTCAGCTCAATCATTTATTGAACGCATGACAAAC TTTGATAAAAATCTTCCAAATGAAAAAGTACTACCAAAACATAGTTTGCTTTATGAGTATTTTACGGTTTATAACGAATTGACAA AGGTCAAATATGTTACTGAAGGAATGCGAAAACCAGCATTTCTTTCAGGTGAACAGAAGAAAGCCATTGTTGATTTACTCTTCA AAACAAATCGAAAAGTAACCGTTAAGCAATTAAAAGAAGATTATTTCAAAAAAATAGAATGTTTTGATAGTGTTGAAATTTCAG GAGTTGAAGATAGATTTAATGCTTCATTAGGTACCTACCATGATTTGCTAAAAATTATTAAAGATAAAGATTTTTTGGATAATG AAGAAAATGAAGATATCTTAGAGGATATTGTTTTAACATTGACCTTATTTGAAGATAGGGAGATGATTGAGGAAAGACTTAAA ACATATGCTCACCTCTTTGATGATAAGGTGATGAAACAGCTTAAACGTCGCCGTTATACTGGTTGGGGACGTTTGTCTCGAAAA TTGATTAATGGTATTAGGGATAAGCAATCTGGCAAAACAATATTAGATTTTTTGAAATCAGATGGTTTTGCCAATCGCAATTTTA TGCAGCTGATCCATGATGATAGTTTGACATTTAAAGAAGACATTCAAAAAGCACAAGTGTCTGGACAAGGCGATAGTTTACAT GAACATATTGCAAATTTAGCTGGTAGCCCTGCTATTAAAAAAGGTATTTTACAGACTGTAAAAGTTGTTGATGAATTGGTCAAA GTAATGGGGCGGCATAAGCCAGAAAATATCGTTATTGAAATGGCACGTGAAAATCAGACAACTCAAAAGGGCCAGAAAAATT CGCGAGAGCGTATGAAACGAATCGAAGAAGGTATCAAAGAATTAGGAAGTCAGATTCTTAAAGAGCATCCTGTTGAAAATAC TCAATTGCAAAATGAAAAGCTCTATCTCTATTATCTCCAAAATGGAAGAGACATGTATGTGGACCAAGAATTAGATATTAATCG TTTAAGTGATTATGATGTCGATCACATTGTTCCACAAAGTTTCCTTAAAGACGATTCAATAGACAATAAGGTCTTAACGCGTTCT 
GATAAAAATCGTGGTAAATCGGATAACGTTCCAAGTGAAGAAGTAGTCAAAAAGATGAAAAACTATTGGAGACAACTTCTAA ACGCCAAGTTAATCACTCAACGTAAGTTTGATAATTTAACGAAAGCTGAACGTGGAGGTTTGAGTGAACTTGATAAAGCTGGT TTTATCAAACGCCAATTGGTTGAAACTCGCCAAATCACTAAGCATGTGGCACAAATTTTGGATAGTCGCATGAATACTAAATAC GATGAAAATGATAAACTTATTCGAGAGGTTAAAGTGATTACCTTAAAATCTAAATTAGTTTCTGACTTCCGAAAAGATTTCCAA TTCTATAAAGTACGTGAGATTAACAATTACCATCATGCCCATGATGCGTATCTAAATGCCGTCGTTGGAACTGCTTTGATTAAG AAATATCCAAAACTTGAATCGGAGTTTGTCTATGGTGATTATAAAGTTTATGATGTTCGTAAAATGATTGCTAAGTCTGAGCAA GAAATAGGCAAAGCAACCGCAAAATATTTCTTTTACTCTAATATCATGAACTTCTTCAAAACAGAAATTACACTTGCAAATGGA GAGATTCGCAAACGCCCTCTAATCGAAACTAATGGGGAAACTGGAGAAATTGTCTGGGATAAAGGGCGAGATTTTGCCACAG TGCGCAAAGTATTGTCCATGCCCCAAGTCAATATTGTCAAGAAAACAGAAGTACAGACAGGCGGATTCTCCAAGGAGTCAATT TTACCAAAAAGAAATTCGGACAAGCTTATTGCTCGTAAAAAAGACTGGGATCCAAAAAAATATGGTGGTTTTGATAGTCCAAC GGTAGCTTATTCAGTCCTAGTGGTTGCTAAGGTGGAAAAAGGGAAATCGAAGAAGTTAAAATCCGTTAAAGAGTTACTAGGG ATCACAATTATGGAAAGAAGTTCCTTTGAAAAAAATCCGATTGACTTTTTAGAAGCTAAAGGATATAAGGAAGTTAAAAAAGA CTTAATCATTAAACTACCTAAATATAGTCTTTTTGAGTTAGAAAACGGTCGTAAACGGATGCTGGCTAGTGCCGGAGAATTACA AAAAGGAAATGAGCTGGCTCTGCCAAGCAAATATGTGAATTTTTTATATTTAGCTAGTCATTATGAAAAGTTGAAGGGTAGTCC AGAAGATAACGAACAAAAACAATTGTTTGTGGAGCAGCATAAGCATTATTTAGATGAGATTATTGAGCAAATCAGTGAATTTT CTAAGCGTGTTATTTTAGCAGATGCCAATTTAGATAAAGTTCTTAGTGCATATAACAAACATAGAGACAAACCAATACGTGAAC AAGCAGAAAATATTATTCATTTATTTACGTTGACGAATCTTGGAGCTCCCGCTGCTTTTTAAATATTTTGATACAACAATTGATCG TAAACGATATACGTCTACAAAAGAAGTTTTAGATGCCACTCTTATCCATCAATCCATCACTGGTCTTTATGAAACACGCATTGAT TTGAGTCAGCTAGGAGGTGACTGATAATCAAATGTCAGACGAAAATGCCAATTATTGAAGCGGCTAACGCCGCTTTTTTTGTTT CTGGTCTCCCTTGCTTGTACTTTACAGTATAGCTTCTAGAGATCTGCAGTGACTGGAGCTTCTAGAGATCTGCAGTGACTGGGA AAACCCTGGCGACTAGTCTTGGACTCCTGTTGATAGATCCAGTAATGACCTCAGAACTCCATCTGGATTTGTTCAGAACGCTCG GTTGCCGCCGGGCGTTTTTTATTGGTGAGAATCCAGGGGTCCCCAATAATTACGATTTAAATCCTTCAAACTTCCCAAAGGCGA GCCCTAGTGACATTAGAAAACCGACTGTAAAAAGTACAGTCGGCATTATCTCATATTATAAAAGCCAGTCATTAGGCCTATCTG ACAATTCCTGAATAGAGTTCATAAACAATCCTGCATGATAACCATCACAAACAGAATGATGTACCTGTAAAGATAGCGGTAAAT ATATTGAATTACCTTTATTAATGAATTTTCCTGCTGTAATAATGGGTAGAAGGTAATTACTATTATTATTGATATTTAAGTTAAAC CCAGTAAATGAAGTCCATGGAATAATAGAAAGAGAAAAAGCATTTTCAGGTATAGGTGTTTTGGGAAACAATTTCCCCGAACC ATTATATTTCTCTACATCAGAAAGGTATAAATCATAAAACTCTTTGAAGTCATTCTTTACAGGAGTCCAAATACCAGAGAATGTT TTAGATACACCATCAAAAATTGTATAAAGTGGCTCTAACTTATCCCAATAACCTAACTCTCCGTCGCTATTGTAACCAGTTCTAA AAGCTGTATTTGAGTTTATCACCCTTGTCACTAAGAAAATAAATGCAGGGTAAAATTTATATCCTTCTTGTTTTATGTTTCGGTAT AAAACACTAATATCAATTTCTGTGGTTATACTAAAAGTCGTTTGTTGGTTCAAATAATGATTAAATATCTCTTTTCTCTTCCAATT GTCTAAATCAATTTTATTAAAGTTCATTTGATATGCCTCCTAAATTTTTTATCTAAAGTGAATTTAGGAGGCTTACTTGTCTGCTTT CTTCATTAGAATCAATCCTTTTTTAAAAGTCAATCCCGTTTGTTGAAGACTTTTGTCCTTTTTCCGCTGCATAAACAATATGGCCCG TTTGTTGAACTACTCTTTAATAAAATAATTTTTCCGTTCCCAATTCCACATTGCAATAATAGAAAATCCATCTTCATCGGCTTTTTC GTCATCATCTGTATGAATCAAATCGCCTTCTTCTGTGTCATCAAGGTTTAATTTTTTTATGTATTTCTTTTAACAAACCACCATAGG AGATTAACCTTTTACGGTGTAAACCTTCCTCCAAATCAGACAAACGTTTCAAATTCTTTTCTTCATCATCGGTCATAAAATCCGTA TCCTTTACAGGATATTTTGCAGTTTCGTCAATTGCCGATTGTATATCCGATTTATATTTATTTTTCGGTCGAATCATTTGAACTTTT ACATTTGGATCATAGTCTAATTTCATTGCCTTTTTCCAAAATTGAATCCATTGTTTTTGATTCACGTAGTTTTCTGTATTCTTAAAA TAAGTTGGTTCCACACATACCAATACATGCATGTGCTGATTATAAGAATTATCTTTATTATTTATTGTCACTTCCGTTGCACGCAT AAAACCAACAAGATTTTTATTAATTTTTTTATATTGCATCATTCGGCGAAATCCTTGAGCCATATCTGACAAACTCTTATTTAATT CTTCGCCATCATAAACATTTTTAACTGTTAATGTGAGAAACAACCAACGAACTGTTGGCTTTTGTTTAATAACTTCAGCAACAAC CTTTTGTGACTGAATGCCATGTTTCATTGCTCTCCTCCAGTTGCACATTGGACAAAGCCTGGATTTACAAAACCACACTCGATAC AACTTTCTTTCGCCTGTTTCACGATTTTGTTTATACTCTAATATTTCAGCACAATCTTTTACTCTTTCAGCCTTTTTAAATTCAAGAA TATGCAGAAGTTCAAAGTAATCAACATTAGCGATTTTTCTTTTTCTCTCCATGGTCTCACTTTTTCCACTTTTTGTCTTGTCCACTAAAA CCCTTGATTTTTCATCTGAATAAATGCTACTATTAGGACACATAATATTAAAAGAAACCCCCATCTATTTAGTTATTTGTTTGGTC ACTTATAACTTTAACAGATGGGGTTTTTCTGTGCAACCAATTTTAAGGGTTTTCCAATACTTTAAAACACATACATACCAACACTT CAACGCACCTTTCAGCAACTAAAATAAAAATGACGTTATTTCTATATGTATCAAGATAAGAAAGAACAAGTTCAAAACCATCAA AAAAAGACACCTTTTCAGGTGCTTTTTTTTATTTTATAAACTCATTCCCTGATCTCGACTTCGTTCTTTTTTTTACCTCTCGGTTATGA GTTAGTTCAAATTCGTTCTTTTTAGGTTCTAAATCGTGTTTTTCTTGGAATTGTGCTGTTTTATCCTTTACCTTGTCTACAAACCCC TTAAAAACGTTTTTAAAGGCTTTTAAGCGTCTGTACGTTCCTTAAGGAATTATTCCTTAGTGCTTTCTAGGTTAATGTCATGATA ATAATGGTTTCTTAGACGTCAGGTGGCACTTTTCGGGGAAATGTCCGCGGAACCCCTATTTGTTTATTTTTCTAAATACATTCAA ATATGTATCCGCTCATGAGACAATAACCCTGATAAATGCTTCAATAATATTGAAAAAGGAAGAGTATGAGTATTCAACATTTCC GTGTCGCCCTTATTCCCTTTTTTGCGGCATTTTGCCTTCCTGTTTTTGCTCACCCAGAAACGCTGGTGAAAGTAAAAGATGCTGA AGATCAGTTGGGTGCACGAGTGGGTTACATCGAACTGGATCTCAACAGCGGTAAGATCCTTGAGAGTTTTCGCCCCGAAGAAC 
GTTTTCCAATGATGAGCACTTTTAAAGTTCTGCTATGTGGCGCGGTATTATCCCGTGTTGACGCCGGGCAAGAGCAACTCGGTC GCCGCATACACTATTCTCAGAATGACTTGGTTGAGTACTCACCAGTCACAGAAAAGCATCTTACGGATGGCATGACAGTAAGA GAATTATGCAGTGCTGCCATAACCATGAGTGATAACACTGCGGCCAACTTACTTCTGACAACGATCGGAGGACCGAAGGAGCT AACCGCTTTTTTTGCACAACATGGGGGATCATGTAACTCGCCTTGATCGTTGGGAACCGGAGCTGAATGAAGCCATACCAAACG ACGAGCGTGACACCACGATGCCTGCAGCAATGGCAACAACGTTGCGCAAACTATTAACTGGCGAACTACTTACTCTAGCTTCCC GGCAACAATTAATAGACTGGATGGAGGCGGATAAAGTTGCAGGACCACTTCTGCGCTCGGCCCTTCCGGCTGGCTGGTTTATT GCTGATAAATCTGGAGCCGGTGAGCGTGGGTCTCGCGGTATCATTGCAGCACTGGGGCCAGATGGTAAGCCCTCCCGTATCG TAGTTATCTACACGACGGGGAGTCAGGCAACTATGGATGAACGAAATAGACAGATCGCTGAGATAGGTGCCTCACTGATTAA GCATTGGTAACTGTCAGACCAAGTTTACTCATATATACTTTAGATTGATTTAAAACTTCATTTTTAATTTAAAAGGATCTAGGTG AAGATCCTTTTTGATAATCTCATGACCAAAATCCCTTAACGTGAGTTTTCGTTCCACTGAGCGTCAGACCCCTTAATAAGATGAT CTTCTTGAGATCGTTTTGGTCTGCGCGTAATCTCTTGCTCTGAAAACGAAAAAACCGCCTTGCAGGGAGGTTTTTCGAAGGTTC TCTGAGCTACCAACTCTTTGAACCGAGGTAACTGGCTTGCAGGAGCGCAGTCACCAAAACTTGTCCTTTCAGTTTAGCCTTAAC CGGCGCATGACTTCAAGACTAACTCCTCTAAATCAATTACCAGTGGCTGCTGCCAGTGGTGCTTTTGCATGTCTTTCCGGGTTG GACTCAAGACGATAGTTACCGGATAAGGCGCAGCGGTCGGACTGAACGGGGGGTTCGTGCATACAGTCCAGCTTGGAGCGA ACTGCCTACCCGGAACTGAGTGTCAGGCGTGGAATGAGACAAACGCGGCCATAACAGCGGAATGACACCGGTAAACCGAAA GGCAGGAACAGGAGAGCGCACGAGGGAGCCGCCAGGGGGAAACGCCTGGTATCTTTATAGTCCTGTCGGGTTTCGCCACCAC TGATTTGAGCGTCAGATTTCGTGATGCTTGTCAGGGGGCGGAGCCTATGGAAAAACGCTTTGCCCGAGCCTATGGAAAAACGC TTTGCCCCACACAGGCTCTTGAACAAGGGATAACAATTTGTCATAGCTGTATTCGTTACAAGGAGGATTTTCGAAAAAACACCC TAACGGGTGTTTTTTTATAGCTGGTCTCCCCAAGTGCTGTAAAAAACGCCGAATGAAGCGGATGAAATTGCTCATCGTCTCGTG AAAAAGCCGAAGAAGGTTAAGCCTGGGTATAAAAAGAAAATGAGCTATGAGATGGAGAAAGCCATAACCATGAGTGATAAC ACTGGTTTTAGAGCTAGAAATAGCAAGTTAAAATAAGGCTAGTCCGTTATCAACTTGAAAAAGTGGCACCGAGTCGGTGCTTT TTTTCTCGGTACCAAATTCCAGAAAAGAGGCCTCCCGAAAGGGGGGCCTTTTTTCGTTTTGGTCCCTCAATGATC

>pCas9g2

tccttatccggttaagatggCTTTTAAATCAATTTTCAGCTCCTGTATACAATTACCAAAGTTTTTCTGAATGAAGCCATGTGTTTTGACA CATTCTATACTCACAAGGAGGTGAGACACatggataagaaatactcaataggctTAGATATCGGCACAAATAGCGTCGGATGGGCGGT GATCACTGATGAATATAAGGTTCCGTCTAAAAAGTTCAAGGTTCTGGGAAATACAGACCGCCACAGTATCAAAAAAAATCTTAT AGGGGCTCTTTTATTTGACAGTGGAGAGACAGCGGAAGCGACTCGTCTCAAACGGACAGCTCGTAGAAGGTATACACGTCGG AAGAATCGTATTTGTTATCTACAGGAGATTTTTTCAAATGAGATGGCGAAAGTAGATGATAGTTTCTTTCATCGACTTGAAGAG TCTTTTTTGGTGGAAGAAGACAAGAAGCATGAACGTCATCCTATTTTTGGAAATATAGTAGATGAAGTTGCTTATCATGAGAAA TATCCAACTATCTATCATCTGCGAAAAAAATTGGTAGATTCTACTGATAAAGCGGATTTGCGCTTAATCTATTTGGCCTTAGCGC ATATGATTAAGTTTCGTGGTCATTTTTTGATTGAGGGAGATTTAAATCCTGATAATAGTGATGTGGACAAACTATTTATCCAGTT GGTACAAACCTACAATCAATTATTTGAAGAAAACCCTATTAACGCAAGTGGAGTAGATGCTAAAGCGATTCTTTCTGCACGATT GAGTAAATCAAGACGATTAGAAAATCTCATTGCTCAGCTCCCCGGTGAGAAGAAAAATGGCTTATTTGGGAATCTCATTGCTTT GTCATTGGGTTTGACCCCTAATTTTAAATCAAATTTTGATTTGGCAGAAGATGCTAAATTACAGCTTTCAAAAGATACTTACGAT GATGATTTAGATAATTTATTGGCGCAAATTGGAGATCAATATGCTGATTTGTTTTTGGCAGCTAAGAATTTATCAGATGCTATTT TACTTTCAGATATCCTAAGAGTAAATACTGAAATAACTAAGGCTCCCCTATCAGCTTCAATGATTAAACGCTACGATGAACATCA TCAAGACTTGACTCTTTTAAAAGCTTTAGTTCGACAACAACTTCCAGAAAAGTATAAAGAAATCTTTTTTTGATCAATCAAAAAAC GGATATGCAGGTTATATTGATGGGGGAGCTAGCCAAGAAGAATTTTATAAATTTATCAAACCAATTTTAGAAAAAATGGATGG TACTGAGGAATTATTGGTGAAACTAAATCGTGAAGATTTGCTGCGCAAGCAACGGACCTTTGACAACGGCTCTATTCCCCATCA AATTCACTTGGGTGAGCTGCATGCTATTTTGAGAAGACAAGAAGACTTTTATCCATTTTTAAAAGACAATCGTGAGAAGATTGA AAAAATCTTGACTTTTCGAATTCCTTATTATGTTGGTCCATTGGCGCGTGGCAATAGTCGTTTTGCATGGATGACTCGGAAGTCT GAAGAAACAATTACCCCATGGAATTTTGAAGAAGTTGTCGATAAAGGTGCTTCAGCTCAATCATTTATTGAACGCATGACAAAC TTTGATAAAAATCTTCCAAATGAAAAAGTACTACCAAAACATAGTTTGCTTTATGAGTATTTTACGGTTTATAACGAATTGACAA AGGTCAAATATGTTACTGAAGGAATGCGAAAACCAGCATTTCTTTCAGGTGAACAGAAGAAAGCCATTGTTGATTTACTCTTCA AAACAAATCGAAAAGTAACCGTTAAGCAATTAAAAGAAGATTATTTCAAAAAAATAGAATGTTTTGATAGTGTTGAAATTTCAG GAGTTGAAGATAGATTTAATGCTTCATTAGGTACCTACCATGATTTGCTAAAAATTATTAAAGATAAAGATTTTTTGGATAATG AAGAAAATGAAGATATCTTAGAGGATATTGTTTTAACATTGACCTTATTTGAAGATAGGGAGATGATTGAGGAAAGACTTAAA ACATATGCTCACCTCTTTGATGATAAGGTGATGAAACAGCTTAAACGTCGCCGTTATACTGGTTGGGGACGTTTGTCTCGAAAA TTGATTAATGGTATTAGGGATAAGCAATCTGGCAAAACAATATTAGATTTTTTGAAATCAGATGGTTTTGCCAATCGCAATTTTA TGCAGCTGATCCATGATGATAGTTTGACATTTAAAGAAGACATTCAAAAAGCACAAGTGTCTGGACAAGGCGATAGTTTACAT GAACATATTGCAAATTTAGCTGGTAGCCCTGCTATTAAAAAAGGTATTTTACAGACTGTAAAAGTTGTTGATGAATTGGTCAAA GTAATGGGGCGGCATAAGCCAGAAAATATCGTTATTGAAATGGCACGTGAAAATCAGACAACTCAAAAGGGCCAGAAAAATT 
CGCGAGAGCGTATGAAACGAATCGAAGAAGGTATCAAAGAATTAGGAAGTCAGATTCTTAAAGAGCATCCTGTTGAAAATAC TCAATTGCAAAATGAAAAGCTCTATCTCTATTATCTCCAAAATGGAAGAGACATGTATGTGGACCAAGAATTAGATATTAATCG TTTAAGTGATTATGATGTCGATCACATTGTTCCACAAAGTTTCCTTAAAGACGATTCAATAGACAATAAGGTCTTAACGCGTTCT GATAAAAATCGTGGTAAATCGGATAACGTTCCAAGTGAAGAAGTAGTCAAAAAGATGAAAAACTATTGGAGACAACTTCTAA ACGCCAAGTTAATCACTCAACGTAAGTTTGATAATTTAACGAAAGCTGAACGTGGAGGTTTGAGTGAACTTGATAAAGCTGGT TTTATCAAACGCCAATTGGTTGAAACTCGCCAAATCACTAAGCATGTGGCACAAATTTTGGATAGTCGCATGAATACTAAATAC GATGAAAATGATAAACTTATTCGAGAGGTTAAAGTGATTACCTTAAAATCTAAATTAGTTTCTGACTTCCGAAAAGATTTCCAA TTCTATAAAGTACGTGAGATTAACAATTACCATCATGCCCATGATGCGTATCTAAATGCCGTCGTTGGAACTGCTTTGATTAAG AAATATCCAAAACTTGAATCGGAGTTTGTCTATGGTGATTATAAAGTTTATGATGTTCGTAAAATGATTGCTAAGTCTGAGCAA GAAATAGGCAAAGCAACCGCAAAATATTTCTTTTACTCTAATATCATGAACTTCTTCAAAACAGAAATTACACTTGCAAATGGA GAGATTCGCAAACGCCCTCTAATCGAAACTAATGGGGAAACTGGAGAAATTGTCTGGGATAAAGGGCGAGATTTTGCCACAG TGCGCAAAGTATTGTCCATGCCCCAAGTCAATATTGTCAAGAAAACAGAAGTACAGACAGGCGGATTCTCCAAGGAGTCAATT TTACCAAAAAGAAATTCGGACAAGCTTATTGCTCGTAAAAAAGACTGGGATCCAAAAAAATATGGTGGTTTTGATAGTCCAAC GGTAGCTTATTCAGTCCTAGTGGTTGCTAAGGTGGAAAAAGGGAAATCGAAGAAGTTAAAATCCGTTAAAGAGTTACTAGGG ATCACAATTATGGAAAGAAGTTCCTTTGAAAAAAATCCGATTGACTTTTTAGAAGCTAAAGGATATAAGGAAGTTAAAAAAGA CTTAATCATTAAACTACCTAAATATAGTCTTTTTGAGTTAGAAAACGGTCGTAAACGGATGCTGGCTAGTGCCGGAGAATTACA AAAAGGAAATGAGCTGGCTCTGCCAAGCAAATATGTGAATTTTTTATATTTAGCTAGTCATTATGAAAAGTTGAAGGGTAGTCC AGAAGATAACGAACAAAAACAATTGTTTGTGGAGCAGCATAAGCATTATTTAGATGAGATTATTGAGCAAATCAGTGAATTTT CTAAGCGTGTTATTTTAGCAGATGCCAATTTAGATAAAGTTCTTAGTGCATATAACAAACATAGAGACAAACCAATACGTGAAC AAGCAGAAAATATTATTCATTTATTTACGTTGACGAATCTTGGAGCTCCCGCTGCTTTTAAATATTTTGATACAACAATTGATCG TAAACGATATACGTCTACAAAAGAAGTTTTAGATGCCACTCTTATCCATCAATCCATCACTGGTCTTTATGAAACACGCATTGAT TTGAGTCAGCTAGGAGGTGACTGATAATCAAATGTCAGACGAAAATGCCAATTATTGAAGCGGCTAACGCCGCTTTTTTTGTTT CTGGTCTCCCTTGCTTGTACTTTACAGTATAGCTTCTAGAGATCTGCAGTGACTGGAGCTTCTAGAGATCAGGTCAGACGCGAT TTCCTGGGTGCTCGAGCCATGGGAAAGCGGTGGTGGAAAAAACGCCGAATGAAGCGGATGAAATTGCTCATCGTCTCGTGAA AAAGCCGAAGAAGGTTAAGCCTGGGTATAAAAAGAAAATGAGCTATGAGATGGAGAAATTGAAGTCATGCGCCGGTTAGTTT TAGAGCTAGAAATAGCAAGTTAAAATAAGGCTAGTCCGTTATCAACTTGAAAAAGTGGCACCGAGTCGGTGCTTTTTTTCTCG GTACCAAATTCCAGAAAAGAGGCCTCCCGAAAGGGGGGCCTTTTTTCGTTTTGGTCCCTCAATGATCCGACAGGAAGTGGTAT TAGCGACTCCTACAAGGAATAGGTGTCTGGGTCATGCAGTGACTGGGAAAACCCTGGCGACTAGTCTTGGACTCCTGTTGATA GATCCAGTAATGACCTCAGAACTCCATCTGGATTTGTTCAGAACGCTCGGTTGCCGCCGGGCGTTTTTTATTGGTGAGAATCCA GGGGTCCCCAATAATTACGATTTAAATCCTTCAAACTTCCCAAAGGCGAGCCCTAGTGACATTAGAAAACCGACTGTAAAAAGT ACAGTCGGCATTATCTCATATTATAAAAGCCAGTCATTAGGCCTATCTGACAATTCCTGAATAGAGTTCATAAACAATCCTGCAT GATAACCATCACAAACAGAATGATGTACCTGTAAAGATAGCGGTAAATATATTGAATTACCTTTATTAATGAATTTTCCTGCTGT AATAATGGGTAGAAGGTAATTACTATTATTATTGATATTTAAGTTAAACCCAGTAAATGAAGTCCATGGAATAATAGAAAGAG AAAAAGCATTTTCAGGTATAGGTGTTTTGGGAAACAATTTCCCCGAACCATTATATTTCTCTACATCAGAAAGGTATAAATCATA AAACTCTTTGAAGTCATTCTTTACAGGAGTCCAAATACCAGAGAATGTTTTAGATACACCATCAAAAATTGTATAAAGTGGCTC TAACTTATCCCAATAACCTAACTCTCCGTCGCTATTGTAACCAGTTCTAAAAGCTGTATTTGAGTTTATCACCCTTGTCACTAAGA AAATAAATGCAGGGTAAAATTTATATCCTTCTTGTTTTATGTTTCGGTATAAAACACTAATATCAATTTCTGTGGTTATACTAAAA GTCGTTTGTTGGTTCAAATAATGATTAAATATCTCTTTTCTCTTCCAATTGTCTAAATCAATTTTATTAAAGTTCATTTGATATGCC TCCTAAATTTTTATCTAAAGTGAATTTAGGAGGCTTACTTGTCTGCTTTCTTCATTAGAATCAATCCTTTTTTAAAAATCAATCCC GTTTGTTGAAGACTTTTGTCCTTTTCCGCTGCATAAACAATATGGCCCGTTTGTTGAACTACTCTTTAATAAAATAATTTTTCCGT TCCCAATTCCACATTGCAATAATAGAAAATCCATCTTCATCGGCTTTTTCGTCATCATCTGTATGAATCAAATCGCCTTCTTCTGT GTCATCAAGGTTTAATTTTTTATGTATTTCTTTTAACAAACCACCATAGGAGATTAACCTTTTACGGTGTAAACCTTCCTCCAAAT CAGACAAACGTTTCAAATTCTTTTCTTCATCATCGGTCATAAAATCCGTATCCTTTACAGGATATTTTGCAGTTTCGTCAATTGCC GATTGTATATCCGATTTATATTTATTTTTCGGTCGAATCATTTGAACTTTTACATTTGGATCATAGTCTAATTTCATTGCCTTTTTC CAAAATTGAATCCATTGTTTTTGATTCACGTAGTTTTCTGTATTCTTAAAATAAGTTGGTTCCACACATACCAATACATGCATGTG CTGATTATAAGAATTATCTTTATTATTTATTGTCACTTCCGTTGCACGCATAAAACCAACAAGATTTTTATTAATTTTTTTATATTG CATCATTCGGCGAAATCCTTGAGCCATATCTGACAAACTCTTATTTAATTCTTCGCCATCATAAACATTTTTAACTGTTAATGTGA GAAACAACCAACGAACTGTTGGCTTTTGTTTAATAACTTCAGCAACAACCTTTTGTGACTGAATGCCATGTTTCATTGCTCTCCT CCAGTTGCACATTGGACAAAGCCTGGATTTACAAAACCACACTCGATACAACTTTTCTTTCGCCTGTTTCACGATTTTGTTTATACT CTAATATTTCAGCACAATCTTTTACTCTTTCAGCCTTTTTAAATTCAAGAATATGCAGAAGTTCAAAGTAATCAACATTAGCGATT TTCTTTTTCTCTCCATGGTCTCACTTTTCCACTTTTTGTCTTGTCCACTAAAACCCTTGATTTTTCATCTGAATAAATGCTACTATTAG GACACATAATATTAAAAGAAACCCCCATCTATTTAGTTATTTGTTTGGTCACTTATAACTTTAACAGATGGGGTTTTTCTGTGCA ACCAATTTTAAGGGTTTTCCAATACTTTAAAACACATACATACCAACACTTCAACGCACCTTTCAGCAACTAAAATAAAAATGAC 
GTTATTTCTATATGTATCAAGATAAGAAAGAACAAGTTCAAAACCATCAAAAAAAGACACCTTTTCAGGTGCTTTTTTTATTTTA TAAACTCATTCCCTGATCTCGACTTCGTTCTTTTTTTACCTCTCGGTTATGAGTTAGTTCAAATTCGTTCTTTTTAGGTTCTAAATC GTGTTTTTCTTGGAATTGTGCTGTTTTATCCTTTACCTTGTCTACAAACCCCTTAAAAACGTTTTTAAAGGCTTTTAAGCGTCTGT ACGTTCCTTAAGGAATTATTCCTTAGTGCTTTCTAGGTTAATGTCATGATAATAATGGTTTCTTAGACGTCAGGTGGCACTTTTC GGGGAAATGTCCGCGGAACCCCTATTTGTTTATTTTTTCTAAATACATTCAAATATGTATCCGCTCATGAGACAATAACCCTGATA AATGCTTCAATAATATTGAAAAAGGAAGAGTATGAGTATTCAACATTTCCGTGTCGCCCTTATTCCCTTTTTTGCGGCATTTTGC CTTCCTGTTTTTGCTCACCCAGAAACGCTGGTGAAAGTAAAAGATGCTGAAGATCAGTTGGGTGCACGAGTGGGTTACATCGA ACTGGATCTCAACAGCGGTAAGATCCTTGAGAGTTTTCGCCCCGAAGAACGTTTTCCAATGATGAGCACTTTTAAAGTTCTGCT ATGTGGCGCGGTATTATCCCGTGTTGACGCCGGGCAAGAGCAACTCGGTCGCCGCATACACTATTCTCAGAATGACTTGGTTG AGTACTCACCAGTCACAGAAAAGCATCTTACGGATGGCATGACAGTAAGAGAATTATGCAGTGCTGCCATAACCATGAGTGAT AACACTGCGGCCAACTTACTTCTGACAACGATCGGAGGACCGAAGGAGCTAACCGCTTTTTTGCACAACATGGGGGATCATGT AACTCGCCTTGATCGTTGGGAACCGGAGCTGAATGAAGCCATACCAAACGACGAGCGTGACACCACGATGCCTGCAGCAATG GCAACAACGTTGCGCAAACTATTAACTGGCGAACTACTTACTCTAGCTTCCCGGCAACAATTAATAGACTGGATGGAGGCGGA TAAAGTTGCAGGACCACTTCTGCGCTCGGCCCTTCCGGCTGGCTGGTTTATTGCTGATAAATCTGGAGCCGGTGAGCGTGGGT CTCGCGGTATCATTGCAGCACTGGGGCCAGATGGTAAGCCCTCCCGTATCGTAGTTATCTACACGACGGGGAGTCAGGCAACT ATGGATGAACGAAATAGACAGATCGCTGAGATAGGTGCCTCACTGATTAAGCATTGGTAACTGTCAGACCAAGTTTACTCATA TATACTTTAGATTGATTTAAAACTTCATTTTTAATTTAAAAGGATCTAGGTGAAGATCCTTTTTGATAATCTCATGACCAAAATCC CTTAACGTGAGTTTTCGTTCCACTGAGCGTCAGACCCCTTAATAAGATGATCTTCTTGAGATCGTTTTGGTCTGCGCGTAATCTC TTGCTCTGAAAACGAAAAAACCGCCTTGCAGGGAGGTTTTTCGAAGGTTCTCTGAGCTACCAACTCTTTGAACCGAGGTAACTG GCTTGCAGGAGCGCAGTCACCAAAACTTGTCCTTTCAGTTTAGCCTTAACCGGCGCATGACTTCAAGACTAACTCCTCTAAATC AATTACCAGTGGCTGCTGCCAGTGGTGCTTTTGCATGTCTTTCCGGGTTGGACTCAAGACGATAGTTACCGGATAAGGCGCAG CGGTCGGACTGAACGGGGGGTTCGTGCATACAGTCCAGCTTGGAGCGAACTGCCTACCCGGAACTGAGTGTCAGGCGTGGAA TGAGACAAACGCGGCCATAACAGCGGAATGACACCGGTAAACCGAAAGGCAGGAACAGGAGAGCGCACGAGGGAGCCGCC AGGGGGAAACGCCTGGTATCTTTATAGTCCTGTCGGGTTTCGCCACCACTGATTTGAGCGTCAGATTTCGTGATGCTTGTCAGG GGGCGGAGCCTATGGAAAAACGCTTTGCCCGAGCCTATGGAAAAACGCTTTGCCCCACACAGGCTCTTGAACAAGGGATAAC AATTTGTCATAGCTGTATTCGTTACAAGGAGGATTTTTGAAAAAACACCCTAACGGGTGTTTTTTTATAGCTGGTCTCCCCAAGT GCTGTAAAAAACGCCGAATGAAGCGGATGAAATTGCTCATCGTCTCGTGAAAAAGCCGAAGAAGGTTAAGCCTGGGTATAAA AAGAAAATGAGCTATGAGATGGAGAAAGCCATAACCATGAGTGATAACACTGGTTTTAGAGCTAGAAATAGCAAGTTAAAAT AAGGCTAGTCCGTTATCAACTTGAAAAAGTGGCACCGAGTCGGTGCTTTTTTTTCTCGGTACCAAATTCCAGAAAAGAGGCCTCC CGAAAGGGGGGCCTTTTTTCGTTTTGGTCCCTCAATGATC

$>$ pCas9g1-D15

tccttatccggttaagatggCTTTTAAATCAATTTTCAGCTCCTGTATACAATTACCAAAGTTTTTCTGAATGAAGCCATGTGTTTTGACA CATTCTATACTCACAAGGAGGTGAGACACatgagtaaatcctggggcaaATTCATCGAGGAGGAAGAGGCGGAAATGGCTTCCCGTC GTAATCTAATGATTGTCGATGGAACTAACTTAGGCTTTCGCTTCAAACATAACAATAGTAAAAAACCATTTGCCTCAAGTTATGT TTCAACTATTCAATCTCTGGCAAAATCCTACTCTGCCAGAACTACGATTGTTCTAGGTGATAAGGGCAAAAGCGTGTTCAGACT AGAACATCTACCAGAGTATAAAGGTAATCGTGATGAAAAGTACGCACAACGTACGGAAGAGGAGAAAGCGCTAGATGAGCA GTTCTTCGAATACCTCAAAGATGCTTTCGAGTTGTGTAAAACTACATTCCCAACTTTTACCATTCGTGGTGTAGAAGCAGATGAC ATGGCGGCGTATATTGTTAAGCTCATCGGGCATCTTTATGATCACGTTTGGCTAATATCTACAGATGGTGACTGGGATACTTTA TTAACGGATAAAGTTTCTCGTTTTTCTTTCACAACACGTCGTGAGTATCATCTTCGTGATATGTATGAGCACCATAACGTGGACG ACGTAGAACAGTTTATCTCCCTGAAAGCAATTATGGGAGATCTAGGAGATAATATTCGTGGTGTTGAAGGAATAGGAGCAAA ACGCGGATATAATATTATTCGTGAGTTTGGTAACGTACTGGATATTATTGATCAGCTTCCACTGCCTGGAAAGCAGAAATATAT ACAGAACCTGAATGCATCGGAAGAACTGCTTTTTCCGAAACTTGATTCTGGTTGATTTACCTACCTACTGTGTGGATGCTATTGCT GCTGTAGGTCAAGATGTGTTAGATAAGTTCACGAAAGACATCCTTGAGATCGCGGAACAATGATAAGCTCTTAAAGGGGGTTT TAGATACCAGAGATGGATAAGAAATACTCAATAGGCTTAGATATCGGCACAAATAGCGTCGGATGGGCGGTGATCACTGATG AATATAAGGTTCCGTCTAAAAAGTTCAAGGTTCTGGGAAATACAGACCGCCACAGTATCAAAAAAAATCTTATAGGGGCTCTTT TATTTGACAGTGGAGAGACAGCGGAAGCGACTCGTCTCAAACGGACAGCTCGTAGAAGGTATACACGTCGGAAGAATCGTAT TTGTTATCTACAGGAGATTTTTTCAAATGAGATGGCGAAAGTAGATGATAGTTTCTTTCATCGACTTGAAGAGTCTTTTTTGGTG GAAGAAGACAAGAAGCATGAACGTCATCCTATTTTTGGAAATATAGTAGATGAAGTTGCTTATCATGAGAAATATCCAACTATC TATCATCTGCGAAAAAAATTGGTAGATTCTACTGATAAAGCGGATTTGCGCTTAATCTATTTGGCCTTAGCGCATATGATTAAG TTTCGTGGTCATTTTTTGATTGAGGGAGATTTAAATCCTGATAATAGTGATGTGGACAAACTATTTATCCAGTTGGTACAAACCT ACAATCAATTATTTGAAGAAAACCCTATTAACGCAAGTGGAGTAGATGCTAAAGCGATTCTTTCTGCACGATTGAGTAAATCAA GACGATTAGAAAATCTCATTGCTCAGCTCCCCGGTGAGAAGAAAAATGGCTTATTTGGGAATCTCATTGCTTTGTCATTGGGTT 
TGACCCCTAATTTTAAATCAAATTTTGATTTGGCAGAAGATGCTAAATTACAGCTTTCAAAAGATACTTACGATGATGATTTAGA TAATTTATTGGCGCAAATTGGAGATCAATATGCTGATTTGTTTTTGGCAGCTAAGAATTTATCAGATGCTATTTTACTTTCAGAT ATCCTAAGAGTAAATACTGAAATAACTAAGGCTCCCCTATCAGCTTCAATGATTAAACGCTACGATGAACATCATCAAGACTTG ACTCTTTTAAAAGCTTTAGTTCGACAACAACTTCCAGAAAAGTATAAAGAAATCTTTTTTGATCAATCAAAAAACGGATATGCA GGTTATATTGATGGGGGAGCTAGCCAAGAAGAATTTTATAAATTTATCAAACCAATTTTAGAAAAAATGGATGGTACTGAGGA ATTATTGGTGAAACTAAATCGTGAAGATTTGCTGCGCAAGCAACGGACCTTTGACAACGGCTCTATTCCCCATCAAATTCACTT GGGTGAGCTGCATGCTATTTTGAGAAGACAAGAAGACTTTTATCCATTTTTAAAAGACAATCGTGAGAAGATTGAAAAAATCT TGACTTTTCGAATTCCTTATTATGTTGGTCCATTGGCGCGTGGCAATAGTCGTTTTGCATGGATGACTCGGAAGTCTGAAGAAA CAATTACCCCATGGAATTTTGAAGAAGTTGTCGATAAAGGTGCTTCAGCTCAATCATTTATTGAACGCATGACAAACTTTGATA AAAATCTTCCAAATGAAAAAGTACTACCAAAACATAGTTTGCTTTATGAGTATTTTACGGTTTATAACGAATTGACAAAGGTCA AATATGTTACTGAAGGAATGCGAAAACCAGCATTTCTTTCAGGTGAACAGAAGAAAGCCATTGTTGATTTACTCTTCAAAACAA ATCGAAAAGTAACCGTTAAGCAATTAAAAGAAGATTATTTCAAAAAAATAGAATGTTTTGATAGTGTTGAAATTTCAGGAGTTG AAGATAGATTTAATGCTTCATTAGGTACCTACCATGATTTGCTAAAAATTATTAAAGATAAAGATTTTTTGGATAATGAAGAAA ATGAAGATATCTTAGAGGATATTGTTTTAACATTGACCTTATTTGAAGATAGGGAGATGATTGAGGAAAGACTTAAAACATAT GCTCACCTCTTTGATGATAAGGTGATGAAACAGCTTAAACGTCGCCGTTATACTGGTTGGGGACGTTTGTCTCGAAAATTGATT AATGGTATTAGGGATAAGCAATCTGGCAAAACAATATTAGATTTTTTGAAATCAGATGGTTTTGCCAATCGCAATTTTATGCAG CTGATCCATGATGATAGTTTGACATTTAAAGAAGACATTCAAAAAGCACAAGTGTCTGGACAAGGCGATAGTTTACATGAACA TATTGCAAATTTAGCTGGTAGCCCTGCTATTAAAAAAGGTATTTTACAGACTGTAAAAGTTGTTGATGAATTGGTCAAAGTAAT GGGGCGGCATAAGCCAGAAAATATCGTTATTGAAATGGCACGTGAAAATCAGACAACTCAAAAGGGCCAGAAAAATTCGCGA GAGCGTATGAAACGAATCGAAGAAGGTATCAAAGAATTAGGAAGTCAGATTCTTAAAGAGCATCCTGTTGAAAATACTCAATT GCAAAATGAAAAGCTCTATCTCTATTATCTCCAAAATGGAAGAGACATGTATGTGGACCAAGAATTAGATATTAATCGTTTAAG TGATTATGATGTCGATCACATTGTTCCACAAAGTTTCCTTAAAGACGATTCAATAGACAATAAGGTCTTAACGCGTTCTGATAAA AATCGTGGTAAATCGGATAACGTTCCAAGTGAAGAAGTAGTCAAAAAGATGAAAAACTATTGGAGACAACTTCTAAACGCCAA GTTAATCACTCAACGTAAGTTTGATAATTTAACGAAAGCTGAACGTGGAGGTTTGAGTGAACTTGATAAAGCTGGTTTTATCAA ACGCCAATTGGTTGAAACTCGCCAAATCACTAAGCATGTGGCACAAATTTTGGATAGTCGCATGAATACTAAATACGATGAAA ATGATAAACTTATTCGAGAGGTTAAAGTGATTACCTTAAAATCTAAATTAGTTTCTGACTTCCGAAAAGATTTCCAATTCTATAA AGTACGTGAGATTAACAATTACCATCATGCCCATGATGCGTATCTAAATGCCGTCGTTGGAACTGCTTTGATTAAGAAATATCC AAAACTTGAATCGGAGTTTGTCTATGGTGATTATAAAGTTTATGATGTTCGTAAAATGATTGCTAAGTCTGAGCAAGAAATAGG CAAAGCAACCGCAAAATATTTCTTTTACTCTAATATCATGAACTTCTTCAAAACAGAAATTACACTTGCAAATGGAGAGATTCGC AAACGCCCTCTAATCGAAACTAATGGGGAAACTGGAGAAATTGTCTGGGATAAAGGGCGAGATTTTGCCACAGTGCGCAAAG TATTGTCCATGCCCCAAGTCAATATTGTCAAGAAAACAGAAGTACAGACAGGCGGATTCTCCAAGGAGTCAATTTTACCAAAA AGAAATTCGGACAAGCTTATTGCTCGTAAAAAAGACTGGGATCCAAAAAAATATGGTGGTTTTGATAGTCCAACGGTAGCTTA TTCAGTCCTAGTGGTTGCTAAGGTGGAAAAAGGGAAATCGAAGAAGTTAAAATCCGTTAAAGAGTTACTAGGGATCACAATTA TGGAAAGAAGTTCCTTTGAAAAAAATCCGATTGACTTTTTAGAAGCTAAAGGATATAAGGAAGTTAAAAAAGACTTAATCATT AAACTACCTAAATATAGTCTTTTTGAGTTAGAAAACGGTCGTAAACGGATGCTGGCTAGTGCCGGAGAATTACAAAAAGGAAA TGAGCTGGCTCTGCCAAGCAAATATGTGAATTTTTTATATTTAGCTAGTCATTATGAAAAGTTGAAGGGTAGTCCAGAAGATAA CGAACAAAAACAATTGTTTGTGGAGCAGCATAAGCATTATTTAGATGAGATTATTGAGCAAATCAGTGAATTTTCTAAGCGTGT TATTTTAGCAGATGCCAATTTAGATAAAGTTCTTAGTGCATATAACAAACATAGAGACAAACCAATACGTGAACAAGCAGAAA ATATTATTCATTTATTTACGTTGACGAATCTTGGAGCTCCCGCTGCTTTTAAATATTTTGATACAACAATTGATCGTAAACGATAT ACGTCTACAAAAGAAGTTTTAGATGCCACTCTTATCCATCAATCCATCACTGGTCTTTATGAAACACGCATTGATTTGAGTCAGC TAGGAGGTGACTGATAATCAAATGTCAGACGAAAATGCCAATTATTGAAGCGGCTAACGCCGCTTTTTTTGTTTCTGGTCTCCC TTGCTTGTACTTTACAGTATAGCTTCTAGAGATCTGCAGTGACTGGAGCTTCTAGAGATCTGCAGTGACTGGGAAAACCCTGGC GACTAGTCTTGGACTCCTGTTGATAGATCCAGTAATGACCTCAGAACTCCATCTGGATTTGTTCAGAACGCTCGGTTGCCGCCG GGCGTTTTTTATTGGTGAGAATCCAGGGGTCCCCAATAATTACGATTTAAATCCTTCAAACTTCCCAAAGGCGAGCCCTAGTGA CATTAGAAAACCGACTGTAAAAAGTACAGTCGGCATTATCTCATATTATAAAAGCCAGTCATTAGGCCTATCTGACAATTCCTG AATAGAGTTCATAAACAATCCTGCATGATAACCATCACAAACAGAATGATGTACCTGTAAAGATAGCGGTAAATATATTGAATT ACCTTTATTAATGAATTTTCCTGCTGTAATAATGGGTAGAAGGTAATTACTATTATTATTGATATTTAAGTTAAACCCAGTAAAT GAAGTCCATGGAATAATAGAAAGAGAAAAAGCATTTTCAGGTATAGGTGTTTTGGGAAACAATTTCCCCGAACCATTATATTTC TCTACATCAGAAAGGTATAAATCATAAAACTCTTTGAAGTCATTCTTTACAGGAGTCCAAATACCAGAGAATGTTTTAGATACA CCATCAAAAATTGTATAAAGTGGCTCTAACTTATCCCAATAACCTAACTCTCCGTCGCTATTGTAACCAGTTCTAAAAGCTGTAT TTGAGTTTATCACCCTTGTCACTAAGAAAATAAATGCAGGGTAAAATTTATATCCTTCTTGTTTTATGTTTCGGTATAAAACACTA ATATCAATTTCTGTGGTTATACTAAAAGTCGTTTGTTGGTTCAAATAATGATTAAATATCTCTTTTCTCTTCCAATTGTCTAAATC AATTTTATTAAAGTTCATTTGATATGCCTCCTAAATTTTTATCTAAAGTGAATTTAGGAGGCTTACTTGTCTGCTTTCTTCATTAG 
AATCAATCCTTTTTTAAAAGTCAATCCCGTTTGTTGAAGACTTTTGTCCTTTTTCCGCTGCATAAACAATATGGCCCGTTTGTTGAA CTACTCTTTAATAAAATAATTTTTCCGTTCCCAATTCCACATTGCAATAATAGAAAATCCATCTTCATCGGCTTTTTCGTCATCATC TGTATGAATCAAATCGCCTTCTTCTGTGTCATCAAGGTTTAATTTTTTATGTATTTCTTTTAACAAACCACCATAGGAGATTAACC TTTTACGGTGTAAACCTTCCTCCAAATCAGACAAACGTTTCAAATTCTTTTCTTCATCATCGGTCATAAAATCCGTATCCTTTACA GGATATTTTGCAGTTTCGTCAATTGCCGATTGTATATCCGATTTATATTTATTTTTCGGTCGAATCATTTGAACTTTTACATTTGG ATCATAGTCTAATTTCATTGCCTTTTTCCAAAATTGAATCCATTGTTTTTGATTCACGTAGTTTTCTGTATTCTTAAAATAAGTTGG TTCCACACATACCAATACATGCATGTGCTGATTATAAGAATTATCTTTATTATTTATTGTCACTTCCGTTGCACGCATAAAACCAA CAAGATTTTTATTAATTTTTTTATATTGCATCATTCGGCGAAATCCTTGAGCCATATCTGACAAACTCTTATTTAATTCTTCGCCAT CATAAACATTTTTAACTGTTAATGTGAGAAACAACCAACGAACTGTTGGCTTTTGTTTAATAACTTCAGCAACAACCTTTTGTGA CTGAATGCCATGTTTCATTGCTCTCCTCCAGTTGCACATTGGACAAAGCCTGGATTTACAAAACCACACTCGATACAACTTTCTT TCGCCTGTTTCACGATTTTGTTTATACTCTAATATTTCAGCACAATCTTTTACTCTTTCAGCCTTTTTAAATTCAAGAATATGCAGA AGTTCAAAGTAATCAACATTAGCGATTTTCTTTTCTCTCCATGGTCTCACTTTTCCACTTTTTTGTCTTGTCCACTAAAACCCTTGAT TTTTCATCTGAATAAATGCTACTATTAGGACACATAATATTAAAAGAAACCCCCATCTATTTAGTTATTTGTTTGGTCACTTATAA CTTTAACAGATGGGGTTTTTCTGTGCAACCAATTTTAAGGGTTTTCCAATACTTTAAAACACATACATACCAACACTTCAACGCA CCTTTCAGCAACTAAAATAAAAATGACGTTATTTCTATATGTATCAAGATAAGAAAGAACAAGTTCAAAACCATCAAAAAAAGA CACCTTTTCAGGTGCTTTTTTTTATTTTATAAACTCATTCCCTGATCTCGACTTCGTTCTTTTTTTTACCTCTCGGTTATGAGTTAGTTC AAATTCGTTCTTTTTAGGTTCTAAATCGTGTTTTTCTTGGAATTGTGCTGTTTTATCCTTTACCTTGTCTACAAACCCCTTAAAAAC GTTTTTAAAGGCTTTTAAGCGTCTGTACGTTCCTTAAGGAATTATTCCTTAGTGCTTTCTAGGTTAATGTCATGATAATAATGGTT TCTTAGACGTCAGGTGGCACTTTTCGGGGAAATGTCCGCGGAACCCCTATTTGTTTATTTTTCTAAATACATTCAAATATGTATC CGCTCATGAGACAATAACCCTGATAAATGCTTCAATAATATTGAAAAAGGAAGAGTATGAGTATTCAACATTTCCGTGTCGCCC TTATTCCCTTTTTTGCGGCATTTTGCCTTCCTGTTTTTGCTCACCCAGAAACGCTGGTGAAAGTAAAAGATGCTGAAGATCAGTT GGGTGCACGAGTGGGTTACATCGAACTGGATCTCAACAGCGGTAAGATCCTTGAGAGTTTTCGCCCCGAAGAACGTTTTCCAA TGATGAGCACTTTTAAAGTTCTGCTATGTGGCGCGGTATTATCCCGTGTTGACGCCGGGCAAGAGCAACTCGGTCGCCGCATA CACTATTCTCAGAATGACTTGGTTGAGTACTCACCAGTCACAGAAAAGCATCTTACGGATGGCATGACAGTAAGAGAATTATG CAGTGCTGCCATAACCATGAGTGATAACACTGCGGCCAACTTACTTCTGACAACGATCGGAGGACCGAAGGAGCTAACCGCTT TTTTGCACAACATGGGGGATCATGTAACTCGCCTTGATCGTTGGGAACCGGAGCTGAATGAAGCCATACCAAACGACGAGCGT GACACCACGATGCCTGCAGCAATGGCAACAACGTTGCGCAAACTATTAACTGGCGAACTACTTACTCTAGCTTCCCGGCAACAA TTAATAGACTGGATGGAGGCGGATAAAGTTGCAGGACCACTTCTGCGCTCGGCCCTTCCGGCTGGCTGGTTTATTGCTGATAA ATCTGGAGCCGGTGAGCGTGGGTCTCGCGGTATCATTGCAGCACTGGGGCCAGATGGTAAGCCCTCCCGTATCGTAGTTATCT ACACGACGGGGAGTCAGGCAACTATGGATGAACGAAATAGACAGATCGCTGAGATAGGTGCCTCACTGATTAAGCATTGGTA ACTGTCAGACCAAGTTTACTCATATATACTTTAGATTGATTTAAAACTTCATTTTTAATTTAAAAGGATCTAGGTGAAGATCCTTT TTGATAATCTCATGACCAAAATCCCTTAACGTGAGTTTTTCGTTCCACTGAGCGTCAGACCCCTTAATAAGATGATCTTCTTGAGA TCGTTTTGGTCTGCGCGTAATCTCTTGCTCTGAAAACGAAAAAACCGCCTTGCAGGGAGGTTTTTCGAAGGTTCTCTGAGCTAC CAACTCTTTGAACCGAGGTAACTGGCTTGCAGGAGCGCAGTCACCAAAACTTGTCCTTTCAGTTTAGCCTTAACCGGCGCATGA CTTCAAGACTAACTCCTCTAAATCAATTACCAGTGGCTGCTGCCAGTGGTGCTTTTTGCATGTCTTTCCGGGTTGGACTCAAGAC GATAGTTACCGGATAAGGCGCAGCGGTCGGACTGAACGGGGGGTTCGTGCATACAGTCCAGCTTGGAGCGAACTGCCTACCC GGAACTGAGTGTCAGGCGTGGAATGAGACAAACGCGGCCATAACAGCGGAATGACACCGGTAAACCGAAAGGCAGGAACAG GAGAGCGCACGAGGGAGCCGCCAGGGGGAAACGCCTGGTATCTTTATAGTCCTGTCGGGTTTCGCCACCACTGATTTGAGCG TCAGATTTCGTGATGCTTGTCAGGGGGCGGAGCCTATGGAAAAACGCTTTGCCCGAGCCTATGGAAAAACGCTTTGCCCCACA CAGGCTCTTGAACAAGGGATAACAATTTGTCATAGCTGTATTCGTTACAAGGAGGATTTTCGAAAAAACACCCTAACGGGTGT TTTTTTATAGCTGGTCTCCCCAAGTGCTGTAAAAAACGCCGAATGAAGCGGATGAAATTGCTCATCGTCTCGTGAAAAAGCCGA AGAAGGTTAAGCCTGGGTATAAAAAGAAAATGAGCTATGAGATGGAGAAAGCCATAACCATGAGTGATAACACTGGTTTTAG AGCTAGAAATAGCAAGTTAAAATAAGGCTAGTCCGTTATCAACTTGAAAAAGTGGCACCGAGTCGGTGCTTTTTTTCTCGGTAC CAAATTCCAGAAAAGAGGCCTCCCGAAAGGGGGGCCTTTTTTTCGTTTTGGTCCCTCAATGATC

$>$ pCas9g2-D15

tccttatccggttaagatggCTTTTAAATCAATTTTCAGCTCCTGTATACAATTACCAAAGTTTTTCTGAATGAAGCCATGTGTTTTGACA CATTCTATACTCACAAGGAGGTGAGACACatgagtaaatcctggggcaaATTCATCGAGGAGGAAGAGGCGGAAATGGCTTCCCGTC GTAATCTAATGATTGTCGATGGAACTAACTTAGGCTTTCGCTTCAAACATAACAATAGTAAAAAACCATTTGCCTCAAGTTATGT TTCAACTATTCAATCTCTGGCAAAATCCTACTCTGCCAGAACTACGATTGTTCTAGGTGATAAGGGCAAAAGCGTGTTCAGACT AGAACATCTACCAGAGTATAAAGGTAATCGTGATGAAAAGTACGCACAACGTACGGAAGAGGAGAAAGCGCTAGATGAGCA GTTCTTCGAATACCTCAAAGATGCTTTCGAGTTGTGTAAAACTACATTCCCAACTTTTACCATTCGTGGTGTAGAAGCAGATGAC ATGGCGGCGTATATTGTTAAGCTCATCGGGCATCTTTATGATCACGTTTGGCTAATATCTACAGATGGTGACTGGGATACTTTA 
TTAACGGATAAAGTTTCTCGTTTTTCTTTCACAACACGTCGTGAGTATCATCTTCGTGATATGTATGAGCACCATAACGTGGACG ACGTAGAACAGTTTATCTCCCTGAAAGCAATTATGGGAGATCTAGGAGATAATATTCGTGGTGTTGAAGGAATAGGAGCAAA ACGCGGATATAATATTATTCGTGAGTTTGGTAACGTACTGGATATTATTGATCAGCTTCCACTGCCTGGAAAGCAGAAATATAT ACAGAACCTGAATGCATCGGAAGAACTGCTTTTCCGAAACTTGATTCTGGTTGATTTACCTACCTACTGTGTGGATGCTATTGCT GCTGTAGGTCAAGATGTGTTAGATAAGTTCACGAAAGACATCCTTGAGATCGCGGAACAATGATAAGCTCTTAAAGGGGGTTT TAGATACCAGAGATGGATAAGAAATACTCAATAGGCTTAGATATCGGCACAAATAGCGTCGGATGGGCGGTGATCACTGATG AATATAAGGTTCCGTCTAAAAAGTTCAAGGTTCTGGGAAATACAGACCGCCACAGTATCAAAAAAAATCTTATAGGGGCTCTTT TATTTGACAGTGGAGAGACAGCGGAAGCGACTCGTCTCAAACGGACAGCTCGTAGAAGGTATACACGTCGGAAGAATCGTAT TTGTTATCTACAGGAGATTTTTTCAAATGAGATGGCGAAAGTAGATGATAGTTTCTTTCATCGACTTGAAGAGTCTTTTTTGGTG GAAGAAGACAAGAAGCATGAACGTCATCCTATTTTTGGAAATATAGTAGATGAAGTTGCTTATCATGAGAAATATCCAACTATC TATCATCTGCGAAAAAAATTGGTAGATTCTACTGATAAAGCGGATTTGCGCTTAATCTATTTGGCCTTAGCGCATATGATTAAG TTTCGTGGTCATTTTTTTGATTGAGGGAGATTTAAATCCTGATAATAGTGATGTGGACAAACTATTTATCCAGTTGGTACAAACCT ACAATCAATTATTTGAAGAAAACCCTATTAACGCAAGTGGAGTAGATGCTAAAGCGATTCTTTCTGCACGATTGAGTAAATCAA GACGATTAGAAAATCTCATTGCTCAGCTCCCCGGTGAGAAGAAAAATGGCTTATTTGGGAATCTCATTGCTTTGTCATTGGGTT TGACCCCTAATTTTAAATCAAATTTTGATTTGGCAGAAGATGCTAAATTACAGCTTTCAAAAGATACTTACGATGATGATTTAGA TAATTTATTGGCGCAAATTGGAGATCAATATGCTGATTTGTTTTTGGCAGCTAAGAATTTATCAGATGCTATTTTACTTTCAGAT ATCCTAAGAGTAAATACTGAAATAACTAAGGCTCCCCTATCAGCTTCAATGATTAAACGCTACGATGAACATCATCAAGACTTG ACTCTTTTAAAAGCTTTAGTTCGACAACAACTTCCAGAAAAGTATAAAGAAATCTTTTTTGATCAATCAAAAAACGGATATGCA GGTTATATTGATGGGGGAGCTAGCCAAGAAGAATTTTATAAATTTATCAAACCAATTTTAGAAAAAATGGATGGTACTGAGGA ATTATTGGTGAAACTAAATCGTGAAGATTTGCTGCGCAAGCAACGGACCTTTGACAACGGCTCTATTCCCCATCAAATTCACTT GGGTGAGCTGCATGCTATTTTGAGAAGACAAGAAGACTTTTATCCATTTTTAAAAGACAATCGTGAGAAGATTGAAAAAATCT TGACTTTTCGAATTCCTTATTATGTTGGTCCATTGGCGCGTGGCAATAGTCGTTTTGCATGGATGACTCGGAAGTCTGAAGAAA CAATTACCCCATGGAATTTTGAAGAAGTTGTCGATAAAGGTGCTTCAGCTCAATCATTTATTGAACGCATGACAAACTTTGATA AAAATCTTCCAAATGAAAAAGTACTACCAAAACATAGTTTGCTTTATGAGTATTTTACGGTTTATAACGAATTGACAAAGGTCA AATATGTTACTGAAGGAATGCGAAAACCAGCATTTCTTTCAGGTGAACAGAAGAAAGCCATTGTTGATTTACTCTTCAAAACAA ATCGAAAAGTAACCGTTAAGCAATTAAAAGAAGATTATTTCAAAAAAATAGAATGTTTTGATAGTGTTGAAATTTCAGGAGTTG AAGATAGATTTAATGCTTCATTAGGTACCTACCATGATTTGCTAAAAATTATTAAAGATAAAGATTTTTTGGATAATGAAGAAA ATGAAGATATCTTAGAGGATATTGTTTTAACATTGACCTTATTTGAAGATAGGGAGATGATTGAGGAAAGACTTAAAACATAT GCTCACCTCTTTGATGATAAGGTGATGAAACAGCTTAAACGTCGCCGTTATACTGGTTGGGGACGTTTGTCTCGAAAATTGATT AATGGTATTAGGGATAAGCAATCTGGCAAAACAATATTAGATTTTTTGAAATCAGATGGTTTTGCCAATCGCAATTTTATGCAG CTGATCCATGATGATAGTTTGACATTTAAAGAAGACATTCAAAAAGCACAAGTGTCTGGACAAGGCGATAGTTTACATGAACA TATTGCAAATTTAGCTGGTAGCCCTGCTATTAAAAAAGGTATTTTACAGACTGTAAAAGTTGTTGATGAATTGGTCAAAGTAAT GGGGCGGCATAAGCCAGAAAATATCGTTATTGAAATGGCACGTGAAAATCAGACAACTCAAAAGGGCCAGAAAAATTCGCGA GAGCGTATGAAACGAATCGAAGAAGGTATCAAAGAATTAGGAAGTCAGATTCTTAAAGAGCATCCTGTTGAAAATACTCAATT GCAAAATGAAAAGCTCTATCTCTATTATCTCCAAAATGGAAGAGACATGTATGTGGACCAAGAATTAGATATTAATCGTTTAAG TGATTATGATGTCGATCACATTGTTCCACAAAGTTTCCTTAAAGACGATTCAATAGACAATAAGGTCTTAACGCGTTCTGATAAA AATCGTGGTAAATCGGATAACGTTCCAAGTGAAGAAGTAGTCAAAAAGATGAAAAACTATTGGAGACAACTTCTAAACGCCAA GTTAATCACTCAACGTAAGTTTGATAATTTAACGAAAGCTGAACGTGGAGGTTTGAGTGAACTTGATAAAGCTGGTTTTATCAA ACGCCAATTGGTTGAAACTCGCCAAATCACTAAGCATGTGGCACAAATTTTGGATAGTCGCATGAATACTAAATACGATGAAA ATGATAAACTTATTCGAGAGGTTAAAGTGATTACCTTAAAATCTAAATTAGTTTCTGACTTCCGAAAAGATTTCCAATTCTATAA AGTACGTGAGATTAACAATTACCATCATGCCCATGATGCGTATCTAAATGCCGTCGTTGGAACTGCTTTGATTAAGAAATATCC AAAACTTGAATCGGAGTTTGTCTATGGTGATTATAAAGTTTATGATGTTCGTAAAATGATTGCTAAGTCTGAGCAAGAAATAGG CAAAGCAACCGCAAAATATTTCTTTTTACTCTAATATCATGAACTTCTTCAAAACAGAAATTACACTTGCAAATGGAGAGATTCGC AAACGCCCTCTAATCGAAACTAATGGGGAAACTGGAGAAATTGTCTGGGATAAAGGGCGAGATTTTGCCACAGTGCGCAAAG TATTGTCCATGCCCCAAGTCAATATTGTCAAGAAAACAGAAGTACAGACAGGCGGATTCTCCAAGGAGTCAATTTTACCAAAA AGAAATTCGGACAAGCTTATTGCTCGTAAAAAAGACTGGGATCCAAAAAAATATGGTGGTTTTGATAGTCCAACGGTAGCTTA TTCAGTCCTAGTGGTTGCTAAGGTGGAAAAAGGGAAATCGAAGAAGTTAAAATCCGTTAAAGAGTTACTAGGGATCACAATTA TGGAAAGAAGTTCCTTTGAAAAAAATCCGATTGACTTTTTAGAAGCTAAAGGATATAAGGAAGTTAAAAAAGACTTAATCATT AAACTACCTAAATATAGTCTTTTTGAGTTAGAAAACGGTCGTAAACGGATGCTGGCTAGTGCCGGAGAATTACAAAAAGGAAA TGAGCTGGCTCTGCCAAGCAAATATGTGAATTTTTTATATTTAGCTAGTCATTATGAAAAGTTGAAGGGTAGTCCAGAAGATAA CGAACAAAAACAATTGTTTGTGGAGCAGCATAAGCATTATTTAGATGAGATTATTGAGCAAATCAGTGAATTTTCTAAGCGTGT TATTTTAGCAGATGCCAATTTAGATAAAGTTCTTAGTGCATATAACAAACATAGAGACAAACCAATACGTGAACAAGCAGAAA ATATTATTCATTTATTTACGTTGACGAATCTTGGAGCTCCCGCTGCTTTTAAATATTTTGATACAACAATTGATCGTAAACGATAT 
ACGTCTACAAAAGAAGTTTTAGATGCCACTCTTATCCATCAATCCATCACTGGTCTTTATGAAACACGCATTGATTTGAGTCAGC TAGGAGGTGACTGATAATCAAATGTCAGACGAAAATGCCAATTATTGAAGCGGCTAACGCCGCTTTTTTTTGTTTCTGGTCTCCC TTGCTTGTACTTTACAGTATAGCTTCTAGAGATCTGCAGTGACTGGAGCTTCTAGAGATCAGGTCAGACGCGATTTCCTGGGTG CTCGAGCCATGGGAAAGCGGTGGTGGAAAAAACGCCGAATGAAGCGGATGAAATTGCTCATCGTCTCGTGAAAAAGCCGAA GAAGGTTAAGCCTGGGTATAAAAAGAAAATGAGCTATGAGATGGAGAAATTGAAGTCATGCGCCGGTTAGTTTTAGAGCTAG AAATAGCAAGTTAAAATAAGGCTAGTCCGTTATCAACTTGAAAAAGTGGCACCGAGTCGGTGCTTTTTTTCTCGGTACCAAATT CCAGAAAAGAGGCCTCCCGAAAGGGGGGCCTTTTTTCGTTTTGGTCCCTCAATGATCCGACAGGAAGTGGTATTAGCGACTCC TACAAGGAATAGGTGTCTGGGTCATGCAGTGACTGGGAAAACCCTGGCGACTAGTCTTGGACTCCTGTTGATAGATCCAGTAA TGACCTCAGAACTCCATCTGGATTTGTTCAGAACGCTCGGTTGCCGCCGGGCGTTTTTTATTGGTGAGAATCCAGGGGTCCCCA ATAATTACGATTTAAATCCTTCAAACTTCCCAAAGGCGAGCCCTAGTGACATTAGAAAACCGACTGTAAAAAGTACAGTCGGCA TTATCTCATATTATAAAAGCCAGTCATTAGGCCTATCTGACAATTCCTGAATAGAGTTCATAAACAATCCTGCATGATAACCATC ACAAACAGAATGATGTACCTGTAAAGATAGCGGTAAATATATTGAATTACCTTTATTAATGAATTTTCCTGCTGTAATAATGGG TAGAAGGTAATTACTATTATTATTGATATTTAAGTTAAACCCAGTAAATGAAGTCCATGGAATAATAGAAAGAGAAAAAGCATT TTCAGGTATAGGTGTTTTGGGAAACAATTTCCCCGAACCATTATATTTCTCTACATCAGAAAGGTATAAATCATAAAACTCTTTG AAGTCATTCTTTACAGGAGTCCAAATACCAGAGAATGTTTTAGATACACCATCAAAAATTGTATAAAGTGGCTCTAACTTATCCC AATAACCTAACTCTCCGTCGCTATTGTAACCAGTTCTAAAAGCTGTATTTGAGTTTATCACCCTTGTCACTAAGAAAATAAATGC AGGGTAAAATTTATATCCTTCTTGTTTTATGTTTCGGTATAAAACACTAATATCAATTTCTGTGGTTATACTAAAAGTCGTTTGTT GGTTCAAATAATGATTAAATATCTCTTTTCTCTTCCAATTGTCTAAATCAATTTTATTAAAGTTCATTTGATATGCCTCCTAAATTT TTATCTAAAGTGAATTTAGGAGGCTTACTTGTCTGCTTTCTTCATTAGAATCAATCCTTTTTTAAAAGTCAATCCCGTTTGTTGAA GACTTTTGTCCTTTTCCGCTGCATAAACAATATGGCCCGTTTGTTGAACTACTCTTTAATAAAATAATTTTTCCGTTCCCAATTCC ACATTGCAATAATAGAAAATCCATCTTCATCGGCTTTTTCGTCATCATCTGTATGAATCAAATCGCCTTCTTCTGTGTCATCAAGG TTTAATTTTTTATGTATTTCTTTTAACAAACCACCATAGGAGATTAACCTTTTTACGGTGTAAACCTTCCTCCAAATCAGACAAACG TTTCAAATTCTTTTCTTCATCATCGGTCATAAAATCCGTATCCTTTACAGGATATTTTGCAGTTTCGTCAATTGCCGATTGTATATC CGATTTATATTTATTTTTTCGGTCGAATCATTTGAACTTTTTACATTTGGATCATAGTCTAATTTCATTGCCTTTTTCCAAAATTGAAT CCATTGTTTTTGATTCACGTAGTTTTCTGTATTCTTAAAATAAGTTGGTTCCACACATACCAATACATGCATGTGCTGATTATAAG AATTATCTTTATTATTTATTGTCACTTCCGTTGCACGCATAAAACCAACAAGATTTTTTATTAATTTTTTTATATTGCATCATTCGGC GAAATCCTTGAGCCATATCTGACAAACTCTTATTTAATTCTTCGCCATCATAAACATTTTTAACTGTTAATGTGAGAAACAACCA ACGAACTGTTGGCTTTTGTTTAATAACTTCAGCAACAACCTTTTGTGACTGAATGCCATGTTTCATTGCTCTCCTCCAGTTGCACA TTGGACAAAGCCTGGATTTACAAAACCACACTCGATACAACTTTCTTTCGCCTGTTTTCACGATTTTGTTTATACTCTAATATTTCA GCACAATCTTTTACTCTTTCAGCCTTTTTTAAATTCAAGAATATGCAGAAGTTCAAAGTAATCAACATTAGCGATTTTCTTTTCTCT CCATGGTCTCACTTTTCCACTTTTTGTCTTGTCCACTAAAACCCTTGATTTTTCATCTGAATAAATGCTACTATTAGGACACATAAT ATTAAAAGAAACCCCCATCTATTTAGTTATTTGTTTGGTCACTTATAACTTTAACAGATGGGGTTTTTCTGTGCAACCAATTTTAA GGGTTTTCCAATACTTTAAAACACATACATACCAACACTTCAACGCACCTTTCAGCAACTAAAATAAAAATGACGTTATTTCTAT ATGTATCAAGATAAGAAAGAACAAGTTCAAAACCATCAAAAAAAGACACCTTTTTCAGGTGCTTTTTTTTATTTTATAAACTCATTC CCTGATCTCGACTTCGTTCTTTTTTTACCTCTCGGTTATGAGTTAGTTCAAATTCGTTCTTTTTAGGTTCTAAATCGTGTTTTTCTT GGAATTGTGCTGTTTTATCCTTTACCTTGTCTACAAACCCCTTAAAAACGTTTTTAAAGGCTTTTAAGCGTCTGTACGTTCCTTAA GGAATTATTCCTTAGTGCTTTCTAGGTTAATGTCATGATAATAATGGTTTCTTAGACGTCAGGTGGCACTTTTCGGGGAAATGT CCGCGGAACCCCTATTTGTTTATTTTTCTAAATACATTCAAATATGTATCCGCTCATGAGACAATAACCCTGATAAATGCTTCAAT AATATTGAAAAAGGAAGAGTATGAGTATTCAACATTTCCGTGTCGCCCTTATTCCCTTTTTTGCGGCATTTTGCCTTCCTGTTTTT GCTCACCCAGAAACGCTGGTGAAAGTAAAAGATGCTGAAGATCAGTTGGGTGCACGAGTGGGTTACATCGAACTGGATCTCA ACAGCGGTAAGATCCTTGAGAGTTTTCGCCCCGAAGAACGTTTTCCAATGATGAGCACTTTTAAAGTTCTGCTATGTGGCGCGG TATTATCCCGTGTTGACGCCGGGCAAGAGCAACTCGGTCGCCGCATACACTATTCTCAGAATGACTTGGTTGAGTACTCACCAG TCACAGAAAAGCATCTTACGGATGGCATGACAGTAAGAGAATTATGCAGTGCTGCCATAACCATGAGTGATAACACTGCGGCC AACTTACTTCTGACAACGATCGGAGGACCGAAGGAGCTAACCGCTTTTTTGCACAACATGGGGGATCATGTAACTCGCCTTGA TCGTTGGGAACCGGAGCTGAATGAAGCCATACCAAACGACGAGCGTGACACCACGATGCCTGCAGCAATGGCAACAACGTTG CGCAAACTATTAACTGGCGAACTACTTACTCTAGCTTCCCGGCAACAATTAATAGACTGGATGGAGGCGGATAAAGTTGCAGG ACCACTTCTGCGCTCGGCCCTTCCGGCTGGCTGGTTTATTGCTGATAAATCTGGAGCCGGTGAGCGTGGGTCTCGCGGTATCAT TGCAGCACTGGGGCCAGATGGTAAGCCCTCCCGTATCGTAGTTATCTACACGACGGGGAGTCAGGCAACTATGGATGAACGA AATAGACAGATCGCTGAGATAGGTGCCTCACTGATTAAGCATTGGTAACTGTCAGACCAAGTTTACTCATATATACTTTAGATT GATTTAAAACTTCATTTTTAATTTAAAAGGATCTAGGTGAAGATCCTTTTTTGATAATCTCATGACCAAAATCCCTTAACGTGAGT TTTCGTTCCACTGAGCGTCAGACCCCTTAATAAGATGATCTTCTTGAGATCGTTTTGGTCTGCGCGTAATCTCTTGCTCTGAAAA CGAAAAAACCGCCTTGCAGGGAGGTTTTTTCGAAGGTTCTCTGAGCTACCAACTCTTTGAACCGAGGTAACTGGCTTGCAGGAG CGCAGTCACCAAAACTTGTCCTTTCAGTTTAGCCTTAACCGGCGCATGACTTCAAGACTAACTCCTCTAAATCAATTACCAGTGG 
CTGCTGCCAGTGGTGCTTTTGCATGTCTTTCCGGGTTGGACTCAAGACGATAGTTACCGGATAAGGCGCAGCGGTCGGACTGA ACGGGGGGTTCGTGCATACAGTCCAGCTTGGAGCGAACTGCCTACCCGGAACTGAGTGTCAGGCGTGGAATGAGACAAACGC GGCCATAACAGCGGAATGACACCGGTAAACCGAAAGGCAGGAACAGGAGAGCGCACGAGGGAGCCGCCAGGGGGAAACGC CTGGTATCTTTATAGTCCTGTCGGGTTTCGCCACCACTGATTTGAGCGTCAGATTTCGTGATGCTTGTCAGGGGGCGGAGCCTA TGGAAAAACGCTTTGCCCGAGCCTATGGAAAAACGCTTTGCCCCACACAGGCTCTTGAACAAGGGATAACAATTTGTCATAGC TGTATTCGTTACAAGGAGGATTTTCGAAAAAACACCCTAACGGGTGTTTTTTTTATAGCTGGTCTCCCCAAGTGCTGTAAAAAAC GCCGAATGAAGCGGATGAAATTGCTCATCGTCTCGTGAAAAAGCCGAAGAAGGTTAAGCCTGGGTATAAAAAGAAAATGAGC TATGAGATGGAGAAAGCCATAACCATGAGTGATAACACTGGTTTTAGAGCTAGAAATAGCAAGTTAAAATAAGGCTAGTCCGT TATCAACTTGAAAAAGTGGCACCGAGTCGGTGCTTTTTTTCTCGGTACCAAATTCCAGAAAAGAGGCCTCCCGAAAGGGGGGC CTTTTTTTCGTTTTGGTCCCTCAATGATC

>pCas9g1-D15-mut GAGTAAATCCTGGGGCAAATTCATCGAGGAGGAAGAGGCGGAAATGGCTTCCCGTCGTAATCTAATGATTGTCGATGGAACT AACTTAGGCTTTCGCTTCAAACATAACAATAGTAAAAAACCATTTGCCTCAAGTTATGTTTCAACTATTCAATCTCTGGCAAAAT CCTACTCTGCCAGAACTACGATTGTTCTAGGTGATAAGGGCAAAAGCGTGTTCAGACTAGAACATCTACCAGAGTATAAAGGT AATCGTGATGAAAAGTACGCACAACGTACGGAAGAGGAGAAAGCGCTAGATGAGCAGTTCTTCGAATACCTCAAAGATGCTT TCGAGTTGTGTAAAACTACATTCCCAACTTTTACCATTCGTGGTGTAGAAGCAGATGACATGGCGGCGTATATTGTTAAGCTCA TCGGGCATCTTTATGATCACGTTTGGCTAATATCTACAGATGGTGACTGGGATACTTTATTAACGGATAAAGTTTCTCGTTTTTC TTTCACAACACGTCGTGAGTATCATCTTCGTGATATGTATGAGCACCATAACGTGGACGACGTAGAACAGTTTATCTCCCTGAA AGCAATTATGGGAGATCTAGGAGATAATATTCGTGGTGTTGAAGGAATAGGAGCAAAACGCGGATATAATATTATTCGTGAG TTTGGTAACGTACTGGATATTATTGATCAGCTTCCACTGCCTGGAAAGCAGAAATATATACAGAACCTGAATGCATCGGAAGA ACTGCTTTTCCGAAACTTGATTCTGGTTGATTTACCTACCTACTGTGTGGATGCTATTGCTGCTGTAGGTCAAGATGTGTTAGAT AAGTTCACGAAAGACATCCTTGAGATCGCGGAACAATGATAAGCTCTTAAAGGGGGTTTTAGATACCAGAGATGGATAAGAA ATACTCAATAGGCTTAGATATCGGCACAAATAGCGTCGGATGGGCGGTGATCACTGATGAATATAAGGTTCCGTCTAAAAAGT TCAAGGTTCTGGGAAATACAGACCGCCACAGTATCAAAAAAAATCTTATAGGGGCTCTTTTATTTGACAGTGGAGAGACAGCG GAAGCGACTCGTCTCAAACGGACAGCTCGTAGAAGGTATACACGTCGGAAGAATCGTATTTGTTATCTACAGGAGATTTTTTC AAATGAGATGGCGAAAGTAGATGATAGTTTCTTTCATCGACTTGAAGAGTCTTTTTTGGTGGAAGAAGACAAGAAGCATGAAC GTCATCCTATTTTTGGAAATATAGTAGATGAAGTTGCTTATCATGAGAAATATCCAACTATCTATCATCTGCGAAAAAAATTGGT AGATTCTACTGATAAAGCGGATTTGCGCTTAATCTATTTGGCCTTAGCGCATATGATTAAGTTTCGTGGTCATTTTTTGATTGAG GGAGATTTAAATCCTGATAATAGTGATGTGGACAAACTATTTATCCAGTTGGTACAAACCTACAATCAATTATTTGAAGAAAAC CCTATTAACGCAAGTGGAGTAGATGCTAAAGCGATTCTTTCTGCACGATTGAGTAAATCAAGACGATTAGAAAATCTCATTGCT CAGCTCCCCGGTGAGAAGAAAAATGGCTTATTTGGGAATCTCATTGCTTTGTCATTGGGTTTGACCCCTAATTTTAAATCAAATT TTGATTTGGCAGAAGATGCTAAATTACAGCTTTCAAAAGATACTTACGATGATGATTTAGATAATTTATTGGCGCAAATTGGAG ATCAATATGCTGATTTGTTTTTGGCAGCTAAGAATTTATCAGATGCTATTTTACTTTCAGATATCCTAAGAGTAAATACTGAAAT AACTAAGGCTCCCCTATCAGCTTCAATGATTAAACGCTACGATGAACATCATCAAGACTTGACTCTTTTAAAAGCTTTAGTTCGA CAACAACTTCCAGAAAAGTATAAAGAAATCTTTTTTGATCAATCAAAAAACGGATATGCAGGTTATATTGATGGGGGAGCTAG CCAAGAAGAATTTTATAAATTTATCAAACCAATTTTAGAAAAAATGGATGGTACTGAGGAATTATTGGTGAAACTAAATCGTGA AGATTTGCTGCGCAAGCAACGGACCTTTGACAACGGCTCTATTCCCCATCAAATTCACTTGGGTGAGCTGCATGCTATTTTGAG AAGACAAGAAGACTTTTATCCATTTTTAAAAGACAATCGTGAGAAGATTGAAAAAATCTTGACTTTTTCGAATTCCTTATTATGTT GGTCCATTGGCGCGTGGCAATAGTCGTTTTGCATGGATGACTCGGAAGTCTGAAGAAACAATTACCCCATGGAATTTTGAAGA AGTTGTCGATAAAGGTGCTTCAGCTCAATCATTTATTGAACGCATGACAAACTTTGATAAAAATCTTCCAAATGAAAAAGTACT ACCAAAACATAGTTTGCTTTATGAGTATTTTACGGTTTATAACGAATTGACAAAGGTCAAATATGTTACTGAAGGAATGCGAAA ACCAGCATTTCTTTCAGGTGAACAGAAGAAAGCCATTGTTGATTTACTCTTCAAAACAAATCGAAAAGTAACCGTTAAGCAATT AAAAGAAGATTATTTCAAAAAAATAGAATGTTTTGATAGTGTTGAAATTTCAGGAGTTGAAGATAGATTTAATGCTTCATTAGG TACCTACCATGATTTGCTAAAAATTATTAAAGATAAAGATTTTTTGGATAATGAAGAAAATGAAGATATCTTAGAGGATATTGT TTTAACATTGACCTTATTTGAAGATAGGGAGATGATTGAGGAAAGACTTAAAACATATGCTCACCTCTTTGATGATAAGGTGAT GAAACAGCTTAAACGTCGCCGTTATACTGGTTGGGGACGTTTGTCTCGAAAATTGATTAATGGTATTAGGGATAAGCAATCTG GCAAAACAATATTAGATTTTTTGAAATCAGATGGTTTTGCCAATCGCAATTTTATGCAGCTGATCCATGATGATAGTTTGACATT TAAAGAAGACATTCAAAAAGCACAAGTGTCTGGACAAGGCGATAGTTTACATGAACATATTGCAAATTTAGCTGGTAGCCCTG CTATTAAAAAAGGTATTTTACAGACTGTAAAAGTTGTTGATGAATTGGTCAAAGTAATGGGGCGGCATAAGCCAGAAAATATC GTTATTGAAATGGCACGTGAAAATCAGACAACTCAAAAGGGCCAGAAAAATTCGCGAGAGCGTATGAAACGAATCGAAGAAG GTATCAAAGAATTAGGAAGTCAGATTCTTAAAGAGCATCCTGTTGAAAATACTCAATTGCAAAATGAAAAGCTCTATCTCTATT ATCTCCAAAATGGAAGAGACATGTATGTGGACCAAGAATTAGATATTAATCGTTTAAGTGATTATGATGTCGATCACATTGTTC 
CACAAAGTTTCCTTAAAGACGATTCAATAGACAATAAGGTCTTAACGCGTTCTGATAAAAATCGTGGTAAATCGGATAACGTTC CAAGTGAAGAAGTAGTCAAAAAGATGAAAAACTATTGGAGACAACTTCTAAACGCCAAGTTAATCACTCAACGTAAGTTTGAT AATTTAACGAAAGCTGAACGTGGAGGTTTGAGTGAACTTGATAAAGCTGGTTTTATCAAACGCCAATTGGTTGAAACTCGCCA AATCACTAAGCATGTGGCACAAATTTTGGATAGTCGCATGAATACTAAATACGATGAAAATGATAAACTTATTCGAGAGGTTA AAGTGATTACCTTAAAATCTAAATTAGTTTCTGACTTCCGAAAAGATTTCCAATTCTATAAAGTACGTGAGATTAACAATTACCA TCATGCCCATGATGCGTATCTAAATGCCGTCGTTGGAACTGCTTTGATTAAGAAATATCCAAAACTTGAATCGGAGTTTGTCTAT GGTGATTATAAAGTTTATGATGTTCGTAAAATGATTGCTAAGTCTGAGCAAGAAATAGGCAAAGCAACCGCAAAATATTTCTTT TACTCTAATATCATGAACTTCTTCAAAACAGAAATTACACTTGCAAATGGAGAGATTCGCAAACGCCCTCTAATCGAAACTAAT GGGGAAACTGGAGAAATTGTCTGGGATAAAGGGCGAGATTTTGCCACAGTGCGCAAAGTATTGTCCATGCCCCAAGTCAATA TTGTCAAGAAAACAGAAGTACAGACAGGCGGATTCTCCAAGGAGTCAATTTTACCAAAAAGAAATTCGGACAAGCTTATTGCT CGTAAAAAAGACTGGGATCCAAAAAAATATGGTGGTTTTGATAGTCCAACGGTAGCTTATTCAGTCCTAGTGGTTGCTAAGGT GGAAAAAGGGAAATCGAAGAAGTTAAAATCCGTTAAAGAGTTACTAGGGATCACAATTATGGAAAGAAGTTCCTTTGAAAAA AATCCGATTGACTTTTTAGAAGCTAAAGGATATAAGGAAGTTAAAAAAGACTTAATCATTAAACTACCTAAATATAGTCTTTTTG AGTTAGAAAACGGTCGTAAACGGATGCTGGCTAGTGCCGGAGAATTACAAAAAGGAAATGAGCTGGCTCTGCCAAGCAAATA TGTGAATTTTTTATATTTAGCTAGTCATTATGAAAAGTTGAAGGGTAGTCCAGAAGATAACGAACAAAAACAATTGTTTGTGGA GCAGCATAAGCATTATTTAGATGAGATTATTGAGCAAATCAGTGAATTTTCTAAGCGTGTTATTTTAGCAGATGCCAATTTAGA TAAAGTTCTTAGTGCATATAACAAACATAGAGACAAACCAATACGTGAACAAGCAGAAAATATTATTCATTTATTTACGTTGAC GAATCTTGGAGCTCCCGCTGCTTTTAAATATTTTGATACAACAATTGATCGTAAACGATATACGTCTACAAAAGAAGTTTTAGAT GCCACTCTTATCCATCAATCCATCACTGGTCTTTATGAAACACGCATTGATTTTGAGTCAGCTAGGAGGTGACTGATAATCAAAT GTCAGACGAAAATGCCAATTATTGAAGCGGCTAACGCCGCTTTTTTTGTTTCTGGTCTCCCTTGCTTGTACTTTACAGTATAGCT TCTAGAGATCTGCAGTGACTGGAGCTTCTAGAGATCTGCAGTGACTGGGAAAACCCTGGCGACTAGTCTTGGACTCCTGTTGA TAGATCCAGTAATGACCTCAGAACTCCATCTGGATTTGTTCAGAACGCTCGGTTGCCGCCGGGCGTTTTTTATTGGTGAGAATC CAGGGGTCCCCAATAATTACGATTTAAATCCTTCAAACTTCCCAAAGGCGAGCCCTAGTGACATTAGAAAACCGACTGTAAAAA GTACAGTCGGCATTATCTCATATTATAAAAGCCAGTCATTAGGCCTATCTGACAATTCCTGAATAGAGTTCATAAACAATCCTGC ATGATAACCATCACAAACAGAATGATGTACCTGTAAAGATAGCGGTAAATATATTGAATTACCTTTATTAATGAATTTTCCTGCT GTAATAATGGGTAGAAGGTAATTACTATTATTATTGATATTTAAGTTAAACCCAGTAAATGAAGTCCATGGAATAATAGAAAGA GAAAAAGCATTTTCAGGTATAGGTGTTTTGGGAAACAATTTCCCCGAACCATTATATTTCTCTACATCAGAAAGGTATAAATCA TAAAACTCTTTGAAGTCATTCTTTACAGGAGTCCAAATACCAGAGAATGTTTTAGATACACCATCAAAAATTGTATAAAGTGGC TCTAACTTATCCCAATAACCTAACTCTCCGTCGCTATTGTAACCAGTTCTAAAAGCTGTATTTGAGTTTATCACCCTTGTCACTAA GAAAATAAATGCAGGGTAAAATTTATATCCTTCTTGTTTTATGTTTCGGTATAAAACACTAATATCAATTTCTGTGGTTATACTA AAAGTCGTTTGTTGGTTCAAATAATGATTAAATATCTCTTTTCTCTTCCAATTGTCTAAATCAATTTTTATTAAAGTTCATTTGATAT GCCTCCTAAATTTTTATCTAAAGTGAATTTAGGAGGCTTACTTGTCTGCTTTCTTCATTAGAATCAATCCTTTTTTAAAAGTCAAT CCCGTTTGTTGAAGACTTTTGTCCTTTTCCGCTGCATAAACAATATGGCCCGTTTGTTGAACTACTCTTTAATAAAATAATTTTTC CGTTCCCAATTCCACATTGCAATAATAGAAAATCCATCTTCATCGGCTTTTTCGTCATCATCTGTATGAATCAAATCGCCTTCTTC TGTGTCATCAAGGTTTAATTTTTTTATGTATTTCTTTTAACAAACCACCATAGGAGATTAACCTTTTACGGTGTAAACCTTCCTCCA AATCAGACAAACGTTTCAAATTCTTTTCTTCATCATCGGTCATAAAATCCGTATCCTTTACAGGATATTTTGCAGTTTCGTCAATT GCCGATTGTATATCCGATTTATATTTATTTTTCGGTCGAATCATTTGAACTTTTACATTTGGATCATAGTCTAATTTCATTGCCTTT TTCCAAAATTGAATCCATTGTTTTTGATTCACGTAGTTTTCTGTATTCTTAAAATAAGTTGGTTCCACACATACCAATACATGCAT GTGCTGATTATAAGAATTATCTTTATTATTTATTGTCACTTCCGTTGCACGCATAAAACCAACAAGATTTTTATTAATTTTTTTATA TTGCATCATTCGGCGAAATCCTTGAGCCATATCTGACAAACTCTTATTTAATTCTTCGCCATCATAAACATTTTTAACTGTTAATG TGAGAAACAACCAACGAACTGTTGGCTTTTGTTTAATAACTTCAGCAACAACCTTTTGTGACTGAATGCCATGTTTCATTGCTCT CCTCCAGTTGCACATTGGACAAAGCCTGGATTTACAAAACCACACTCGATACAACTTTCTTTTGGCTGTTTCACGATTTTGTTTAT ACTCTAATATTTCAGCACAATCTTTTACTCTTTCAGCCTTTTTAAATTCAAGAATATGCAGAAGTTCAAAGTAATCAACATTAGCG ATTTTCTTTTCTCTCCATGGTCTCACTTTTCCACTTTTTGTCTTGTCCACTAAAACCCTTGATTTTTCATCTGAATAAATGCTACTAT TAGGACACATAATATTAAAAGAAACCCCCATCTATTTAGTTATTTGTTTGGTCACTTATAACTTTAACAGATGGGGTTTTTCTGT GCAACCAATTTTAAGGGTTTTCCAATACTTTAAAACACATACATACCAACACTTCAACGCACCTTTCAGCAACTAAAATAAAAAT GACGTTATTTCTATATGTATCAAGATAAGAAAGAACAAGTTCAAAACCATCAAAAAAAGACACCTTTTCAGGTGCTTTTTTTATT TTATAAACTCATTCCCTGATCTCGACTTCGTTCTTTTTTTACCTCTCGGTTATGAGTTAGTTCAAATTCGTTCTTTTTAGGTTCTAA ATCGTGTTTTTCTTGGAATTGTGCTGTTTTATCCTTTACCTTGTCTACAAACCCCTTAAAAACGTTTTTTAAAGGCTTTTTAAGCGTCT GTACGTTCCTTAAGGAATTATTCCTTAGTGCTTTCTAGGTTAATGTCATGATAATAATGGTTTCTTAGACGTCAGGTGGCACTTT TCGGGGAAATGTCCGCGGAACCCCTATTTGTTTATTTTTCTAAATACATTCAAATATGTATCCGCTCATGAGACAATAACCCTGA TAAATGCTTCAATAATATTGAAAAAGGAAGAGTATGAGTATTCAACATTTCCGTGTCGCCCTTATTCCCTTTTTTGCGGCATTTT GCCTTCCTGTTTTTGCTCACCCAGAAACGCTGGTGAAAGTAAAAGATGCTGAAGATCAGTTGGGTGCACGAGTGGGTTACATC 
GAACTGGATCTCAACAGCGGTAAGATCCTTGAGAGTTTTCGCCCCGAAGAACGTTTTTCCAATGATGAGCACTTTTAAAGTTCTG CTATGTGGCGCGGTATTATCCCGTGTTGACGCCGGGCAAGAGCAACTCGGTCGCCGCATACACTATTCTCAGAATGACTTGGTT GAGTACTCACCAGTCACAGAAAAGCATCTTACGGATGGCATGACAGTAAGAGAATTATGCAGTGCTGCCATAACCATGAGTGA TAACACTGCGGCCAACTTACTTCTGACAACGATCGGAGGACCGAAGGAGCTAACCGCTTTTTTGCACAACATGGGGGATCATG TAACTCGCCTTGATCGTTGGGAACCGGAGCTGAATGAAGCCATACCAAACGACGAGCGTGACACCACGATGCCTGCAGCAAT GGCAACAACGTTGCGCAAACTATTAACTGGCGAACTACTTACTCTAGCTTCCCGGCAACAATTAATAGACTGGATGGAGGCGG ATAAAGTTGCAGGACCACTTCTGCGCTCGGCCCTTCCGGCTGGCTGGTTTATTGCTGATAAATCTGGAGCCGGTGAGCGTGGG TCTCGCGGTATCATTGCAGCACTGGGGCCAGATGGTAAGCCCTCCCGTATCGTAGTTATCTACACGACGGGGAGTCAGGCAAC TATGGATGAACGAAATAGACAGATCGCTGAGATAGGTGCCTCACTGATTAAGCATTGGTAACTGTCAGACCAAGTTTACTCAT ATATACTTTAGATTGATTTAAAACTTCATTTTTAATTTAAAAGGATCTAGGTGAAGATCCTTTTTGATAATCTCATGACCAAAATC CCTTAACGTGAGTTTTCGTTCCACTGAGCGTCAGACCCCTTAATAAGATGATCTTCTTGAGATCGTTTTTGGTCTGCGCGTAATCT CTTGCTCTGAAAACGAAAAAACCGCCTTGCAGGGAGGTTTTTCGAAGGTTCTCTGAGCTACCAACTCTTTGAACCGAGGTAACT GGCTTGCAGGAGCGCAGTCACCAAAACTTGTCCTTTCAGTTTAGCCTTAACCGGCGCATGACTTCAAGACTAACTCCTCTAAAT CAATTACCAGTGGCTGCTGCCAGTGGTGCTTTTGCATGTCTTTCCGGGTTGGACTCAAGACGATAGTTACCGGATAAGGCGCA GCGGTCGGACTGAACGGGGGGTTCGTGCATACAGTCCAGCTTGGAGCGAACTGCCTACCCGGAACTGAGTGTCAGGCGTGGA ATGAGACAAACGCGGCCATAACAGCGGAATGACACCGGTAAACCGAAAGGCAGGAACAGGAGAGCGCACGAGGGAGCCGC CAGGGGGAAACGCCTGGTATCTTTATAGTCCTGTCGGGTTTCGCCACCACTGATTTGAGCGTCAGATTTCGTGATGCTTGTCAG GGGGCGGAGCCTATGGAAAAACGCTTTGCCCGAGCCTATGGAAAAACGCTTTGCCCCACACAGGCTCTTGAACAAGGGATAA CAATTTGTCATAGCTGTATTCGTTACAAGGAGGATTTTCGAAAAAACACCCTAACGGGTGTTTTTTTATAGCTGGTCTCCCCAAG TGCTGTAAAAAACGCCGAATGAAGCGGATGAAATTGCTCATCGTCTCGTGAAAAAGCCGAAGAAGGTTAAGCCTGGGTATAA AAAGAAAATGAGCTATGAGATGGAGAAAGCCATAACCATGAGTGATAACACTGGTTTTAGAGCTAGAAATAGCAAGTTAAAA TAAGGCTAGTCCGTTATCAACTTGAAAAAGTGGCACCGAGTCGGTGCTTTTTTTCTCGGTACCAAATTCCAGAAAAGAGGCCTC CCGAAAGGGGGGCCTTTTTTCGTTTTGGTCCCTCAATGATCTCCTTATCCGGTTAAGATGGC

>pCas9g2-D15-mut

GAGTAAATCCTGGGGCAAATTCATCGAGGAGGAAGAGGCGGAAATGGCTTCCCGTCGTAATCTAATGATTGTCGATGGAACT AACTTAGGCTTTCGCTTCAAACATAACAATAGTAAAAAACCATTTGCCTCAAGTTATGTTTCAACTATTCAATCTCTGGCAAAAT CCTACTCTGCCAGAACTACGATTGTTCTAGGTGATAAGGGCAAAAGCGTGTTCAGACTAGAACATCTACCAGAGTATAAAGGT AATCGTGATGAAAAGTACGCACAACGTACGGAAGAGGAGAAAGCGCTAGATGAGCAGTTCTTCGAATACCTCAAAGATGCTT TCGAGTTGTGTAAAACTACATTCCCAACTTTTACCATTCGTGGTGTAGAAGCAGATGACATGGCGGCGTATATTGTTAAGCTCA TCGGGCATCTTTATGATCACGTTTGGCTAATATCTACAGATGGTGACTGGGATACTTTATTAACGGATAAAGTTTCTCGTTTTTC TTTCACAACACGTCGTGAGTATCATCTTCGTGATATGTATGAGCACCATAACGTGGACGACGTAGAACAGTTTATCTCCCTGAA AGCAATTATGGGAGATCTAGGAGATAATATTCGTGGTGTTGAAGGAATAGGAGCAAAACGCGGATATAATATTATTCGTGAG TTTGGTAACGTACTGGATATTATTGATCAGCTTCCACTGCCTGGAAAGCAGAAATATATACAGAACCTGAATGCATCGGAAGA ACTGCTTTTCCGAAACTTGATTCTGGTTGATTTACCTACCTACTGTGTGGATGCTATTGCTGCTGTAGGTCAAGATGTGTTAGAT AAGTTCACGAAAGACATCCTTGAGATCGCGGAACAATGATAAGCTCTTAAAGGGGGTTTTAGATACCAGAGATGGATAAGAA ATACTCAATAGGCTTAGATATCGGCACAAATAGCGTCGGATGGGCGGTGATCACTGATGAATATAAGGTTCCGTCTAAAAAGT TCAAGGTTCTGGGAAATACAGACCGCCACAGTATCAAAAAAAATCTTATAGGGGCTCTTTTATTTGACAGTGGAGAGACAGCG GAAGCGACTCGTCTCAAACGGACAGCTCGTAGAAGGTATACACGTCGGAAGAATCGTATTTGTTATCTACAGGAGATTTTTTC AAATGAGATGGCGAAAGTAGATGATAGTTTCTTTCATCGACTTGAAGAGTCTTTTTTGGTGGAAGAAGACAAGAAGCATGAAC GTCATCCTATTTTTGGAAATATAGTAGATGAAGTTGCTTATCATGAGAAATATCCAACTATCTATCATCTGCGAAAAAAATTGGT AGATTCTACTGATAAAGCGGATTTGCGCTTAATCTATTTGGCCTTAGCGCATATGATTAAGTTTCGTGGTCATTTTTTGATTGAG GGAGATTTAAATCCTGATAATAGTGATGTGGACAAACTATTTATCCAGTTGGTACAAACCTACAATCAATTATTTGAAGAAAAC CCTATTAACGCAAGTGGAGTAGATGCTAAAGCGATTCTTTCTGCACGATTGAGTAAATCAAGACGATTAGAAAATCTCATTGCT CAGCTCCCCGGTGAGAAGAAAAATGGCTTATTTGGGAATCTCATTGCTTTGTCATTGGGTTTGACCCCTAATTTTAAATCAAATT TTGATTTGGCAGAAGATGCTAAATTACAGCTTTCAAAAGATACTTACGATGATGATTTAGATAATTTATTGGCGCAAATTGGAG ATCAATATGCTGATTTGTTTTTGGCAGCTAAGAATTTATCAGATGCTATTTTACTTTCAGATATCCTAAGAGTAAATACTGAAAT AACTAAGGCTCCCCTATCAGCTTCAATGATTAAACGCTACGATGAACATCATCAAGACTTGACTCTTTTTAAAAGCTTTAGTTCGA CAACAACTTCCAGAAAAGTATAAAGAAATCTTTTTTTGATCAATCAAAAAACGGATATGCAGGTTATATTGATGGGGGAGCTAG CCAAGAAGAATTTTATAAATTTATCAAACCAATTTTAGAAAAAATGGATGGTACTGAGGAATTATTGGTGAAACTAAATCGTGA AGATTTGCTGCGCAAGCAACGGACCTTTGACAACGGCTCTATTCCCCATCAAATTCACTTGGGTGAGCTGCATGCTATTTTGAG AAGACAAGAAGACTTTTATCCATTTTTAAAAGACAATCGTGAGAAGATTGAAAAAATCTTGACTTTTCGAATTCCTTATTATGTT GGTCCATTGGCGCGTGGCAATAGTCGTTTTGCATGGATGACTCGGAAGTCTGAAGAAACAATTACCCCATGGAATTTTGAAGA 
AGTTGTCGATAAAGgTGCTTCAGCTCAATCATTTATTGAACGCATGACAAACTTTGATAAAAATCTTCCAAATGAAAAAGTACT ACCAAAACATAGTTTGCTTTATGAGTATTTTACGGTTTATAACGAATTGACAAAGGTCAAATATGTTACTGAAGGAATGCGAAA ACCAGCATTTCTTTCAGGTGAACAGAAGAAAGCCATTGTTGATTTACTCTTCAAAACAAATCGAAAAGTAACCGTTAAGCAATT AAAAGAAGATTATTTCAAAAAAATAGAATGTTTTGATAGTGTTGAAATTTCAGGAGTTGAAGATAGATTTAATGCTTCATTAGG TACCTACCATGATTTGCTAAAAATTATTAAAGATAAAGATTTTTTGGATAATGAAGAAAATGAAGATATCTTAGAGGATATTGT TTTAACATTGACCTTATTTGAAGATAGGGAGATGATTGAGGAAAGACTTAAAACATATGCTCACCTCTTTGATGATAAGGTGAT GAAACAGCTTAAACGTCGCCGTTATACTGGTTGGGGACGTTTGTCTCGAAAATTGATTAATGGTATTAGGGATAAGCAATCTG GCAAAACAATATTAGATTTTTTGAAATCAGATGGTTTTGCCAATCGCAATTTTATGCAGCTGATCCATGATGATAGTTTGACATT TAAAGAAGACATTCAAAAAGCACAAGTGTCTGGACAAGGCGATAGTTTACATGAACATATTGCAAATTTAGCTGGTAGCCCTG CTATTAAAAAAGGTATTTTACAGACTGTAAAAGTTGTTGATGAATTGGTCAAAGTAATGGGGCGGCATAAGCCAGAAAATATC GTTATTGAAATGGCACGTGAAAATCAGACAACTCAAAAGGGCCAGAAAAATTCGCGAGAGCGTATGAAACGAATCGAAGAAG GTATCAAAGAATTAGGAAGTCAGATTCTTAAAGAGCATCCTGTTGAAAATACTCAATTGCAAAATGAAAAGCTCTATCTCTATT ATCTCCAAAATGGAAGAGACATGTATGTGGACCAAGAATTAGATATTAATCGTTTAAGTGATTATGATGTCGATCACATTGTTC CACAAAGTTTCCTTAAAGACGATTCAATAGACAATAAGGTCTTAACGCGTTCTGATAAAAATCGTGGTAAATCGGATAACGTTC CAAGTGAAGAAGTAGTCAAAAAGATGAAAAACTATTGGAGACAACTTCTAAACGCCAAGTTAATCACTCAACGTAAGTTTGAT AATTTAACGAAAGCTGAACGTGGAGGTTTGAGTGAACTTGATAAAGCTGGTTTTATCAAACGCCAATTGGTTGAAACTCGCCA AATCACTAAGCATGTGGCACAAATTTTGGATAGTCGCATGAATACTAAATACGATGAAAATGATAAACTTATTCGAGAGGTTA AAGTGATTACCTTAAAATCTAAATTAGTTTCTGACTTCCGAAAAGATTTCCAATTCTATAAAGTACGTGAGATTAACAATTACCA TCATGCCCATGATGCGTATCTAAATGCCGTCGTTGGAACTGCTTTGATTAAGAAATATCCAAAACTTGAATCGGAGTTTGTCTAT GGTGATTATAAAGTTTATGATGTTCGTAAAATGATTGCTAAGTCTGAGCAAGAAATAGGCAAAGCAACCGCAAAATATTTCTTT TACTCTAATATCATGAACTTCTTCAAAACAGAAATTACACTTGCAAATGGAGAGATTCGCAAACGCCCTCTAATCGAAACTAAT GGGGAAACTGGAGAAATTGTCTGGGATAAAGGGCGAGATTTTGCCACAGTGCGCAAAGTATTGTCCATGCCCCAAGTCAATA TTGTCAAGAAAACAGAAGTACAGACAGGCGGATTCTCCAAGGAGTCAATTTTACCAAAAAGAAATTCGGACAAGCTTATTGCT CGTAAAAAAGACTGGGATCCAAAAAAATATGGTGGTTTTGATAGTCCAACGGTAGCTTATTCAGTCCTAGTGGTTGCTAAGGT GGAAAAAGGGAAATCGAAGAAGTTAAAATCCGTTAAAGAGTTACTAGGGATCACAATTATGGAAAGAAGTTCCTTTGAAAAA AATCCGATTGACTTTTTAGAAGCTAAAGGATATAAGGAAGTTAAAAAAGACTTAATCATTAAACTACCTAAATATAGTCTTTTTG AGTTAGAAAACGGTCGTAAACGGATGCTGGCTAGTGCCGGAGAATTACAAAAAGGAAATGAGCTGGCTCTGCCAAGCAAATA TGTGAATTTTTTATATTTAGCTAGTCATTATGAAAAGTTGAAGGGTAGTCCAGAAGATAACGAACAAAAACAATTGTTTGTGGA GCAGCATAAGCATTATTTAGATGAGATTATTGAGCAAATCAGTGAATTTTCTAAGCGTGTTATTTTAGCAGATGCCAATTTAGA TAAAGTTCTTAGTGCATATAACAAACATAGAGACAAACCAATACGTGAACAAGCAGAAAATATTATTCATTTATTTACGTTGAC GAATCTTGGAGCTCCCGCTGCTTTTAAATATTTTGATACAACAATTGATCGTAAACGATATACGTCTACAAAAGAAGTTTTAGAT GCCACTCTTATCCATCAATCCATCACTGGTCTTTATGAAACACGCATTGATTTGAGTCAGCTAGGAGGTGACTGATAATCAAAT GTCAGACGAAAATGCCAATTATTGAAGCGGCTAACGCCGCTTTTTTTGTTTCTGGTCTCCCTTGCTTGTACTTTACAGTATAGCT TCTAGAGATCTGCAGTGACTGGAGCTTCTAGAGATCAGGTCAGACGCGATTTCCTGGGTGCTCGAGCCATGGGAAAGCGGTG GTGGAAAAAACGCCGAATGAAGCGGATGAAATTGCTCATCGTCTCGTGAAAAAGCCGAAGAAGGTTAAGCCTGGGTATAAAA AGAAAATGAGCTATGAGATGGAGAAATTGAAGTCATGCGCCGGTTAGTTTTAGAGCTAGAAATAGCAAGTTAAAATAAGGCT AGTCCGTTATCAACTTGAAAAAGTGGCACCGAGTCGGTGCTTTTTTTCTCGGTACCAAATTCCAGAAAAGAGGCCTCCCGAAAG GGGGGCCTTTTTTCGTTTTGGTCCCTCAATGATCCGACAGGAAGTGGTATTAGCGACTCCTACAAGGAATAGGTGTCTGGGTC ATGCAGTGACTGGGAAAACCCTGGCGACTAGTCTTGGACTCCTGTTGATAGATCCAGTAATGACCTCAGAACTCCATCTGGATT TGTTCAGAACGCTCGGTTGCCGCCGGGCGTTTTTTATTGGTGAGAATCCAGGGGTCCCCAATAATTACGATTTAAATCCTTCAA ACTTCCCAAAGGCGAGCCCTAGTGACATTAGAAAACCGACTGTAAAAAGTACAGTCGGCATTATCTCATATTATAAAAGCCAGT CATTAGGCCTATCTGACAATTCCTGAATAGAGTTCATAAACAATCCTGCATGATAACCATCACAAACAGAATGATGTACCTGTA AAGATAGCGGTAAATATATTGAATTACCTTTATTAATGAATTTTCCTGCTGTAATAATGGGTAGAAGGTAATTACTATTATTATT GATATTTAAGTTAAACCCAGTAAATGAAGTCCATGGAATAATAGAAAGAGAAAAAGCATTTTCAGGTATAGGTGTTTTGGGAA ACAATTTCCCCGAACCATTATATTTCTCTACATCAGAAAGGTATAAATCATAAAACTCTTTGAAGTCATTCTTTACAGGAGTCCA AATACCAGAGAATGTTTTAGATACACCATCAAAAATTGTATAAAGTGGCTCTAACTTATCCCAATAACCTAACTCTCCGTCGCTA TTGTAACCAGTTCTAAAAGCTGTATTTGAGTTTATCACCCTTGTCACTAAGAAAATAAATGCAGGGTAAAATTTATATCCTTCTT GTTTTATGTTTCGGTATAAAACACTAATATCAATTTCTGTGGTTATACTAAAAGTCGTTTGTTGGTTCAAATAATGATTAAATATC TCTTTTCTCTTCCAATTGTCTAAATCAATTTTATTAAAGTTCATTTGATATGCCTCCTAAATTTTTATCTAAAGTGAATTTAGGAGG CTTACTTGTCTGCTTTCTTCATTAGAATCAATCCTTTTTTAAAAGTCAATCCCGTTTGTTGAAGACTTTTGTCCTTTTCCGCTGCAT AAACAATATGGCCCGTTTGTTGAACTACTCTTTAATAAAATAATTTTTCCGTTCCCAATTCCACATTGCAATAATAGAAAATCCAT CTTCATCGGCTTTTTCGTCATCATCTGTATGAATCAAATCGCCTTCTTCTGTGTCATCAAGGTTTAATTTTTTATGTATTTCTTTTA ACAAACCACCATAGGAGATTAACCTTTTACGGTGTAAACCTTCCTCCAAATCAGACAAACGTTTCAAATTCTTTTCTTCATCATC 
GGTCATAAAATCCGTATCCTTTACAGGATATTTTGCAGTTTCGTCAATTGCCGATTGTATATCCGATTTATATTTATTTTTCGGTC GAATCATTTGAACTTTTACATTTGGATCATAGTCTAATTTCATTGCCTTTTTTCCAAAATTGAATCCATTGTTTTTTGATTCACGTAGT TTTCTGTATTCTTAAAATAAGTTGGTTCCACACATACCAATACATGCATGTGCTGATTATAAGAATTATCTTTATTATTTATTGTC ACTTCCGTTGCACGCATAAAACCAACAAGATTTTTATTAATTTTTTTTATATTGCATCATTCGGCGAAATCCTTGAGCCATATCTGA CAAACTCTTATTTAATTCTTCGCCATCATAAACATTTTTAAACTGTTAATGTGAGAAACAACCAACGAACTGTTGGCTTTTGTTTAA TAACTTCAGCAACAACCTTTTTGTGACTGAATGCCATGTTTCATTGCTCTCCTCCAGTTGCACATTGGACAAAGCCTGGATTTACA AAACCACACTCGATACAACTTTCTTTCGCCTGTTTCACGATTTTGTTTATACTCTAATATTTCAGCACAATCTTTTACTCTTTCAGC CTTTTTAAATTCAAGAATATGCAGAAGTTCAAAGTAATCAACATTAGCGATTTTCTTTTCTCTCCATGGTCTCACTTTTCCACTTTT TGTCTTGTCCACTAAAACCCTTGATTTTTCATCTGAATAAATGCTACTATTAGGACACATAATATTAAAAGAAACCCCCATCTATT TAGTTATTTGTTTGGTCACTTATAACTTTAACAGATGGGGTTTTTCTGTGCAACCAATTTTAAGGGTTTTCCAATACTTTAAAACA CATACATACCAACACTTCAACGCACCTTTCAGCAACTAAAATAAAAATGACGTTATTTCTATATGTATCAAGATAAGAAAGAAC AAGTTCAAAACCATCAAAAAAAGACACCTTTTCAGGTGCTTTTTTTATTTTTATAAACTCATTCCCTGATCTCGACTTCGTTCTTTTT TTACCTCTCGGTTATGAGTTAGTTCAAATTCGTTCTTTTTAGGTTTCTAAATCGTGTTTTTCTTGGAATTGTGCTGTTTTATCCTTTA CCTTGTCTACAAACCCCTTAAAAACGTTTTTAAAGGCTTTTAAGCGTCTGTACGTTCCTTAAGGAATTATTCCTTAGTGCTTTCTA GGTTAATGTCATGATAATAATGGTTTCTTAGACGTCAGGTGGCACTTTTCGGGGAAATGTCCGCGGAACCCCTATTTGTTTATT TTTCTAAATACATTCAAATATGTATCCGCTCATGAGACAATAACCCTGATAAATGCTTCAATAATATTGAAAAAGGAAGAGTAT GAGTATTCAACATTTCCGTGTCGCCCTTATTCCCTTTTTTTGCGGCATTTTGCCTTCCTGTTTTTGCTCACCCAGAAACGCTGGTGA AAGTAAAAGATGCTGAAGATCAGTTGGGTGCACGAGTGGGTTACATCGAACTGGATCTCAACAGCGGTAAGATCCTTGAGAG TTTTCGCCCCGAAGAACGTTTTCCAATGATGAGCACTTTTAAAGTTCTGCTATGTGGCGCGGTATTATCCCGTGTTGACGCCGG GCAAGAGCAACTCGGTCGCCGCATACACTATTCTCAGAATGACTTGGTTGAGTACTCACCAGTCACAGAAAAGCATCTTACGG ATGGCATGACAGTAAGAGAATTATGCAGTGCTGCCATAACCATGAGTGATAACACTGCGGCCAACTTACTTCTGACAACGATC GGAGGACCGAAGGAGCTAACCGCTTTTTTGCACAACATGGGGGATCATGTAACTCGCCTTGATCGTTGGGAACCGGAGCTGA ATGAAGCCATACCAAACGACGAGCGTGACACCACGATGCCTGCAGCAATGGCAACAACGTTGCGCAAACTATTAACTGGCGA ACTACTTACTCTAGCTTCCCGGCAACAATTAATAGACTGGATGGAGGCGGATAAAGTTGCAGGACCACTTCTGCGCTCGGCCCT TCCGGCTGGCTGGTTTATTGCTGATAAATCTGGAGCCGGTGAGCGTGGGTCTCGCGGTATCATTGCAGCACTGGGGCCAGATG GTAAGCCCTCCCGTATCGTAGTTATCTACACGACGGGGAGTCAGGCAACTATGGATGAACGAAATAGACAGATCGCTGAGAT AGGTGCCTCACTGATTAAGCATTGGTAACTGTCAGACCAAGTTTACTCATATATACTTTAGATTGATTTAAAACTTCATTTTTAAT TTAAAAGGATCTAGGTGAAGATCCTTTTTGATAATCTCATGACCAAAATCCCTTAACGTGAGTTTTCGTTCCACTGAGCGTCAGA CCCCTTAATAAGATGATCTTCTTGAGATCGTTTTGGTCTGCGCGTAATCTCTTGCTCTGAAAACGAAAAAACCGCCTTGCAGGG AGGTTTTTCGAAGGTTCTCTGAGCTACCAACTCTTTGAACCGAGGTAACTGGCTTGCAGGAGCGCAGTCACCAAAACTTGTCCT TTCAGTTTAGCCTTAACCGGCGCATGACTTCAAGACTAACTCCTCTAAATCAATTACCAGTGGCTGCTGCCAGTGGTGCTTTTGC ATGTCTTTCCGGGTTGGACTCAAGACGATAGTTACCGGATAAGGCGCAGCGGTCGGACTGAACGGGGGGTTCGTGCATACAG TCCAGCTTGGAGCGAACTGCCTACCCGGAACTGAGTGTCAGGCGTGGAATGAGACAAACGCGGCCATAACAGCGGAATGACA CCGGTAAACCGAAAGGCAGGAACAGGAGAGCGCACGAGGGAGCCGCCAGGGGGAAACGCCTGGTATCTTTATAGTCCTGTC GGGTTTCGCCACCACTGATTTGAGCGTCAGATTTCGTGATGCTTGTCAGGGGGCGGAGCCTATGGAAAAACGCTTTGCCCGAG CCTATGGAAAAACGCTTTGCCCCACACAGGCTCTTGAACAAGGGATAACAATTTGTCATAGCTGTATTCGTTACAAGGAGGATT TTCGAAAAAACACCCTAACGGGTGTTTTTTTATAGCTGGTCTCCCCAAGTGCTGTAAAAAACGCCGAATGAAGCGGATGAAATT GCTCATCGTCTCGTGAAAAAGCCGAAGAAGGTTAAGCCTGGGTATAAAAAGAAAATGAGCTATGAGATGGAGAAAGCCATAA CCATGAGTGATAACACTGGTTTTAGAGCTAGAAATAGCAAGTTAAAATAAGGCTAGTCCGTTATCAACTTGAAAAAGTGGCAC CGAGTCGGTGCTTTTTTTCTCGGTACCAAATTCCAGAAAAGAGGCCTCCCGAAAGGGGGGCCTTTTTTTCGTTTTGGTCCCTCAA TGATCTCCTTATCCGGTTAAGATGGC

>pCas9g1-D15-GFP

GTGACTGGGAAAACCCTGGCGACTAGTCTTGGACTCCTGTTGATAGATCCAGTAATGACCTCAGAACTCCATCTGGATTTGTTC AGAACGCTCGGTTGCCGCCGGGCGTTTTTTTATTGGTGAGAATCCAGGGGTCCCCAATAATTACGATTTAAATCCTTCAAACTTC CCAAAGGCGAGCCCTAGTGACATTAGAAAACCGACTGTAAAAAGTACAGTCGGCATTATCTCATATTATAAAAGCCAGTCATT AGGCCTATCTGACAATTCCTGAATAGAGTTCATAAACAATCCTGCATGATAACCATCACAAACAGAATGATGTACCTGTAAAGA TAGCGGTAAATATATTGAATTACCTTTATTAATGAATTTTCCTGCTGTAATAATGGGTAGAAGGTAATTACTATTATTATTGATA TTTAAGTTAAACCCAGTAAATGAAGTCCATGGAATAATAGAAAGAGAAAAAGCATTTTCAGGTATAGGTGTTTTGGGAAACAA TTTCCCCGAACCATTATATTTCTCTACATCAGAAAGGTATAAATCATAAAACTCTTTGAAGTCATTCTTTACAGGAGTCCAAATA CCAGAGAATGTTTTAGATACACCATCAAAAATTGTATAAAGTGGCTCTAACTTATCCCAATAACCTAACTCTCCGTCGCTATTGT AACCAGTTCTAAAAGCTGTATTTGAGTTTATCACCCTTGTCACTAAGAAAATAAATGCAGGGTAAAATTTATATCCTTCTTGTTT TATGTTTCGGTATAAAACACTAATATCAATTTCTGTGGTTATACTAAAAGTCGTTTGTTGGTTCAAATAATGATTAAATATCTCTT 
TTCTCTTCCAATTGTCTAAATCAATTTTATTAAAGTTCATTTGATATGCCTCCTAAATTTTTATCTAAAGTGAATTTAGGAGGCTT ACTTGTCTGCTTTCTTCATTAGAATCAATCCTTTTTTAAAAAGTCAATCCCGTTTGTTGAAGACTTTTGTCCTTTTCCGCTGCATAAA CAATATGGCCCGTTTGTTGAACTACTCTTTAATAAAATAATTTTTTCCGTTCCCAATTCCACATTGCAATAATAGAAAATCCATCTT CATCGGCTTTTTCGTCATCATCTGTATGAATCAAATCGCCTTCTTCTGTGTCATCAAGGTTTAATTTTTTATGTATTTCTTTTAACA AACCACCATAGGAGATTAACCTTTTACGGTGTAAACCTTCCTCCAAATCAGACAAACGTTTCAAATTCTTTTCTTCATCATCGGTC ATAAAATCCGTATCCTTTACAGGATATTTTGCAGTTTCGTCAATTGCCGATTGTATATCCGATTTATATTTATTTTTCGGTCGAAT CATTTGAACTTTTACATTTGGATCATAGTCTAATTTCATTGCCTTTTTCCAAAATTGAATCCATTGTTTTTGATTCACGTAGTTTTC TGTATTCTTAAAATAAGTTGGTTCCACACATACCAATACATGCATGTGCTGATTATAAGAATTATCTTTATTATTTATTGTCACTT CCGTTGCACGCATAAAACCAACAAGATTTTTATTAATTTTTTTTATATTGCATCATTCGGCGAAATCCTTGAGCCATATCTGACAA ACTCTTATTTAATTCTTCGCCATCATAAACATTTTTAACTGTTAATGTGAGAAACAACCAACGAACTGTTGGCTTTTGTTTAATAA CTTCAGCAACAACCTTTTGTGACTGAATGCCATGTTTCATTGCTCTCCTCCAGTTGCACATTGGACAAAGCCTGGATTTACAAAA CCACACTCGATACAACTTTCTTTCGCCTGTTTCACGATTTTGTTTATACTCTAATATTTCAGCACAATCTTTTACTCTTTCAGCCTTT TTAAATTCAAGAATATGCAGAAGTTCAAAGTAATCAACATTAGCGATTTTCTTTTCTCTCCATGGTCTCACTTTTCCACTTTTTGT CTTGTCCACTAAAACCCTTGATTTTTCATCTGAATAAATGCTACTATTAGGACACATAATATTAAAAGAAACCCCCATCTATTTAG TTATTTGTTTGGTCACTTATAACTTTAACAGATGGGGTTTTTCTGTGCAACCAATTTTAAGGGTTTTCCAATACTTTAAAACACAT ACATACCAACACTTCAACGCACCTTTCAGCAACTAAAATAAAAATGACGTTATTTCTATATGTATCAAGATAAGAAAGAACAAG TTCAAAACCATCAAAAAAAGACACCTTTTCAGGTGCTTTTTTTATTTTATAAACTCATTCCCTGATCTCGACTTCGTTCTTTTTTTA CCTCTCGGTTATGAGTTAGTTCAAATTCGTTCTTTTTAGGTTCTAAATCGTGTTTTTCTTGGAATTGTGCTGTTTTATCCTTTACCT TGTCTACAAACCCCTTAAAAACGTTTTTAAAGGCTTTTAAGCGTCTGTACGTTCCTTAAGGAATTATTCCTTAGTGCTTTCTAGGT TAATGTCATGATAATAATGGTTTCTTAGACGTCAGGTGGCACTTTTCGGGGAAATGTCCGCGGAACCCCTATTTGTTTATTTTTC TAAATACATTCAAATATGTATCCGCTCATGAGACAATAACCCTGATAAATGCTTCAATAATATTGAAAAAGGAAGAGTATGAGT ATTCAACATTTCCGTGTCGCCCTTATTCCCTTTTTTGCGGCATTTTGCCTTCCTGTTTTTTGCTCACCCAGAAACGCTGGTGAAAGT AAAAGATGCTGAAGATCAGTTGGGTGCACGAGTGGGTTACATCGAACTGGATCTCAACAGCGGTAAGATCCTTGAGAGTTTTC GCCCCGAAGAACGTTTTCCAATGATGAGCACTTTTAAAGTTCTGCTATGTGGCGCGGTATTATCCCGTGTTGACGCCGGGCAAG AGCAACTCGGTCGCCGCATACACTATTCTCAGAATGACTTGGTTGAGTACTCACCAGTCACAGAAAAGCATCTTACGGATGGC ATGACAGTAAGAGAATTATGCAGTGCTGCCATAACCATGAGTGATAACACTGCGGCCAACTTACTTCTGACAACGATCGGAGG ACCGAAGGAGCTAACCGCTTTTTTGCACAACATGGGGGATCATGTAACTCGCCTTGATCGTTGGGAACCGGAGCTGAATGAAG CCATACCAAACGACGAGCGTGACACCACGATGCCTGCAGCAATGGCAACAACGTTGCGCAAACTATTAACTGGCGAACTACTT ACTCTAGCTTCCCGGCAACAATTAATAGACTGGATGGAGGCGGATAAAGTTGCAGGACCACTTCTGCGCTCGGCCCTTCCGGC TGGCTGGTTTATTGCTGATAAATCTGGAGCCGGTGAGCGTGGGTCTCGCGGTATCATTGCAGCACTGGGGCCAGATGGTAAG CCCTCCCGTATCGTAGTTATCTACACGACGGGGAGTCAGGCAACTATGGATGAACGAAATAGACAGATCGCTGAGATAGGTG CCTCACTGATTAAGCATTGGTAACTGTCAGACCAAGTTTACTCATATATACTTTAGATTGATTTAAAACTTCATTTTTAATTTAAA AGGATCTAGGTGAAGATCCTTTTTGATAATCTCATGACCAAAATCCCTTAACGTGAGTTTTCGTTCCACTGAGCGTCAGACCCCT TAATAAGATGATCTTCTTGAGATCGTTTTGGTCTGCGCGTAATCTCTTGCTCTGAAAACGAAAAAACCGCCTTGCAGGGAGGTT TTTCGAAGGTTCTCTGAGCTACCAACTCTTTGAACCGAGGTAACTGGCTTGCAGGAGCGCAGTCACCAAAACTTGTCCTTTCAG TTTAGCCTTAACCGGCGCATGACTTCAAGACTAACTCCTCTAAATCAATTACCAGTGGCTGCTGCCAGTGGTGCTTTTGCATGTC TTTCCGGGTTGGACTCAAGACGATAGTTACCGGATAAGGCGCAGCGGTCGGACTGAACGGGGGGTTCGTGCATACAGTCCAG CTTGGAGCGAACTGCCTACCCGGAACTGAGTGTCAGGCGTGGAATGAGACAAACGCGGCCATAACAGCGGAATGACACCGGT AAACCGAAAGGCAGGAACAGGAGAGCGCACGAGGGAGCCGCCAGGGGGAAACGCCTGGTATCTTTATAGTCCTGTCGGGTT TCGCCACCACTGATTTGAGCGTCAGATTTCGTGATGCTTGTCAGGGGGCGGAGCCTATGGAAAAACGCTTTGCCCGAGCCTAT GGAAAAACGCTTTGCCCCACACAGGCTCTTGAACAAGGGATAACAATTTGTCATAGCTGTATTCGTTACAAGGAGGATTTTCG AAAAAACACCCTAACGGGTGTTTTTTTATAGCTGGTCTCCCCAAGTGCTGTAAAAAACGCCGAATGAAGCGGATGAAATTGCT CATCGTCTCGTGAAAAAGCCGAAGAAGGTTAAGCCTGGGTATAAAAAGAAAATGAGCTATGAGATGGAGAAAGCCATAACCA TGAGTGATAACACTGGTTTTAGAGCTAGAAATAGCAAGTTAAAATAAGGCTAGTCCGTTATCAACTTGAAAAAGTGGCACCGA GTCGGTGCTTTTTTTCTCGGTACCAAATTCCAGAAAAGAGGCCTCCCGAAAGGGGGGCCTTTTTTCGTTTTTGGTCCCTCAATGA TCTCCTTATCCGGTTAAGATGGCTTTTAAATCAATTTTCAGCTCCTGTATACAATTACCAAAGTTTTTCTGAATGAAGCCATGTGT TTTGACACATTCTATACTCACAAGGAGGTGAGACACATGAGTAAATCCTGGGGCAAATTCATCGAGGAGGAAGAGGCGGAAA TGGCTTCCCGTCGTAATCTAATGATTGTCGATGGAACTAACTTAGGCTTTCGCTTCAAACATAACAATAGTAAAAAACCATTTGC CTCAAGTTATGTTTCAACTATTCAATCTCTGGCAAAATCCTACTCTGCCAGAACTACGATTGTTCTAGGTGATAAGGGCAAAAG CGTGTTCAGACTAGAACATCTACCAGAGTATAAAGGTAATCGTGATGAAAAGTACGCACAACGTACGGAAGAGGAGAAAGCG CTAGATGAGCAGTTCTTCGAATACCTCAAAGATGCTTTCGAGTTGTGTAAAACTACATTCCCAACTTTTACCATTCGTGGTGTAG AAGCAGATGACATGGCGGCGTATATTGTTAAGCTCATCGGGCATCTTTATGATCACGTTTGGCTAATATCTACAGATGGTGACT GGGATACTTTATTAACGGATAAAGTTTCTCGTTTTTCTTTCACAACACGTCGTGAGTATCATCTTCGTGATATGTATGAGCACCA 
TAACGTGGACGACGTAGAACAGTTTATCTCCCTGAAAGCAATTATGGGAGATCTAGGAGATAATATTCGTGGTGTTGAAGGAA TAGGAGCAAAACGCGGATATAATATTATTCGTGAGTTTGGTAACGTACTGGATATTATTGATCAGCTTCCACTGCCTGGAAAGC AGAAATATATACAGAACCTGAATGCATCGGAAGAACTGCTTTTCCGAAACTTGATTCTGGTTGATTTACCTACCTACTGTGTGG ATGCTATTGCTGCTGTAGGTCAAGATGTGTTAGATAAGTTCACGAAAGACATCCTTGAGATCGCGGAACAATGATAAGCTCTT AAAGGGGGTTTTAGATACCAGAGATGGATAAGAAATACTCAATAGGCTTAGATATCGGCACAAATAGCGTCGGATGGGCGGT GATCACTGATGAATATAAGGTTCCGTCTAAAAAGTTCAAGGTTCTGGGAAATACAGACCGCCACAGTATCAAAAAAAATCTTAT AGGGGCTCTTTTATTTGACAGTGGAGAGACAGCGGAAGCGACTCGTCTCAAACGGACAGCTCGTAGAAGGTATACACGTCGG AAGAATCGTATTTGTTATCTACAGGAGATTTTTTCAAATGAGATGGCGAAAGTAGATGATAGTTTCTTTCATCGACTTGAAGAG TCTTTTTTTGGTGGAAGAAGACAAGAAGCATGAACGTCATCCTATTTTTGGAAATATAGTAGATGAAGTTGCTTATCATGAGAAA TATCCAACTATCTATCATCTGCGAAAAAAATTGGTAGATTCTACTGATAAAGCGGATTTGCGCTTAATCTATTTGGCCTTAGCGC ATATGATTAAGTTTCGTGGTCATTTTTTGATTGAGGGAGATTTAAATCCTGATAATAGTGATGTGGACAAACTATTTATCCAGTT GGTACAAACCTACAATCAATTATTTGAAGAAAACCCTATTAACGCAAGTGGAGTAGATGCTAAAGCGATTCTTTCTGCACGATT GAGTAAATCAAGACGATTAGAAAATCTCATTGCTCAGCTCCCCGGTGAGAAGAAAAATGGCTTATTTGGGAATCTCATTGCTTT GTCATTGGGTTTGACCCCTAATTTTAAATCAAATTTTGATTTGGCAGAAGATGCTAAATTACAGCTTTCAAAAGATACTTACGAT GATGATTTAGATAATTTATTGGCGCAAATTGGAGATCAATATGCTGATTTGTTTTTGGCAGCTAAGAATTTATCAGATGCTATTT TACTTTCAGATATCCTAAGAGTAAATACTGAAATAACTAAGGCTCCCCTATCAGCTTCAATGATTAAACGCTACGATGAACATCA TCAAGACTTGACTCTTTTTAAAAGCTTTAGTTCGACAACAACTTCCAGAAAAGTATAAAGAAATCTTTTTTGGATCAATCAAAAAAC GGATATGCAGGTTATATTGATGGGGGAGCTAGCCAAGAAGAATTTTATAAATTTATCAAACCAATTTTAGAAAAAATGGATGG TACTGAGGAATTATTGGTGAAACTAAATCGTGAAGATTTGCTGCGCAAGCAACGGACCTTTGACAACGGCTCTATTCCCCATCA AATTCACTTGGGTGAGCTGCATGCTATTTTGAGAAGACAAGAAGACTTTTATCCATTTTTTAAAAGACAATCGTGAGAAGATTGA AAAAATCTTGACTTTTCGAATTCCTTATTATGTTGGTCCATTGGCGCGTGGCAATAGTCGTTTTGCATGGATGACTCGGAAGTCT GAAGAAACAATTACCCCATGGAATTTTGGAGAAGTTGTCGATAAAGGTGCTTCAGCTCAATCATTTATTGAACGCATGACAAAC TTTGATAAAAATCTTCCAAATGAAAAAGTACTACCAAAACATAGTTTGCTTTATGAGTATTTTACGGTTTATAACGAATTGACAA AGGTCAAATATGTTACTGAAGGAATGCGAAAACCAGCATTTCTTTTCAGGTGAACAGAAGAAAGCCATTGTTGATTTACTCTTCA AAACAAATCGAAAAGTAACCGTTAAGCAATTAAAAGAAGATTATTTCAAAAAAATAGAATGTTTTGATAGTGTTGAAATTTCAG GAGTTGAAGATAGATTTAATGCTTCATTAGGTACCTACCATGATTTGCTAAAAATTATTAAAGATAAAGATTTTTTGGATAATG AAGAAAATGAAGATATCTTAGAGGATATTGTTTTAACATTGACCTTATTTGAAGATAGGGAGATGATTGAGGAAAGACTTAAA ACATATGCTCACCTCTTTGATGATAAGGTGATGAAACAGCTTAAACGTCGCCGTTATACTGGTTGGGGACGTTTGTCTCGAAAA TTGATTAATGGTATTAGGGATAAGCAATCTGGCAAAACAATATTAGATTTTTTGAAATCAGATGGTTTTGCCAATCGCAATTTTA TGCAGCTGATCCATGATGATAGTTTGACATTTAAAGAAGACATTCAAAAAGCACAAGTGTCTGGACAAGGCGATAGTTTACAT GAACATATTGCAAATTTAGCTGGTAGCCCTGCTATTAAAAAAGGTATTTTACAGACTGTAAAAGTTGTTGATGAATTGGTCAAA GTAATGGGGCGGCATAAGCCAGAAAATATCGTTATTGAAATGGCACGTGAAAATCAGACAACTCAAAAGGGCCAGAAAAATT CGCGAGAGCGTATGAAACGAATCGAAGAAGGTATCAAAGAATTAGGAAGTCAGATTCTTAAAGAGCATCCTGTTGAAAATAC TCAATTGCAAAATGAAAAGCTCTATCTCTATTATCTCCAAAATGGAAGAGACATGTATGTGGACCAAGAATTAGATATTAATCG TTTAAGTGATTATGATGTCGATCACATTGTTCCACAAAGTTTCCTTAAAGACGATTCAATAGACAATAAGGTCTTAACGCGTTCT GATAAAAATCGTGGTAAATCGGATAACGTTCCAAGTGAAGAAGTAGTCAAAAAGATGAAAAACTATTGGAGACAACTTCTAA ACGCCAAGTTAATCACTCAACGTAAGTTTGATAATTTAACGAAAGCTGAACGTGGAGGTTTGAGTGAACTTGATAAAGCTGGT TTTATCAAACGCCAATTGGTTGAAACTCGCCAAATCACTAAGCATGTGGCACAAATTTTGGATAGTCGCATGAATACTAAATAC GATGAAAATGATAAACTTATTCGAGAGGTTAAAGTGATTACCTTAAAATCTAAATTAGTTTCTGACTTCCGAAAAGATTTCCAA TTCTATAAAGTACGTGAGATTAACAATTACCATCATGCCCATGATGCGTATCTAAATGCCGTCGTTGGAACTGCTTTGATTAAG AAATATCCAAAACTTGAATCGGAGTTTGTCTATGGTGATTATAAAGTTTATGATGTTCGTAAAATGATTGCTAAGTCTGAGCAA GAAATAGGCAAAGCAACCGCAAAATATTTCTTTTACTCTAATATCATGAACTTCTTCAAAACAGAAATTACACTTGCAAATGGA GAGATTCGCAAACGCCCTCTAATCGAAACTAATGGGGAAACTGGAGAAATTGTCTGGGATAAAGGGCGAGATTTTGCCACAG TGCGCAAAGTATTGTCCATGCCCCAAGTCAATATTGTCAAGAAAACAGAAGTACAGACAGGCGGATTCTCCAAGGAGTCAATT TTACCAAAAAGAAATTCGGACAAGCTTATTGCTCGTAAAAAAGACTGGGATCCAAAAAAATATGGTGGTTTTGATAGTCCAAC GGTAGCTTATTCAGTCCTAGTGGTTGCTAAGGTGGAAAAAGGGAAATCGAAGAAGTTAAAATCCGTTAAAGAGTTACTAGGG ATCACAATTATGGAAAGAAGTTCCTTTGAAAAAAATCCGATTGACTTTTTAGAAGCTAAAGGATATAAGGAAGTTAAAAAAGA CTTAATCATTAAACTACCTAAATATAGTCTTTTTGAGTTAGAAAACGGTCGTAAACGGATGCTGGCTAGTGCCGGAGAATTACA AAAAGGAAATGAGCTGGCTCTGCCAAGCAAATATGTGAATTTTTTATATTTAGCTAGTCATTATGAAAAGTTGAAGGGTAGTCC AGAAGATAACGAACAAAAACAATTGTTTGTGGAGCAGCATAAGCATTATTTAGATGAGATTATTGAGCAAATCAGTGAATTTT CTAAGCGTGTTATTTTAGCAGATGCCAATTTAGATAAAGTTCTTAGTGCATATAACAAACATAGAGACAAACCAATACGTGAAC AAGCAGAAAATATTATTCATTTATTTACGTTGACGAATCTTGGAGCTCCCGCTGCTTTTAAATATTTTGATACAACAATTGATCG TAAACGATATACGTCTACAAAAGAAGTTTTAGATGCCACTCTTATCCATCAATCCATCACTGGTCTTTATGAAACACGCATTGAT 
TTGAGTCAGCTAGGAGGTGACTGATAATCAAATGTCAGACGAAAATGCCAATTATTGAAGCGGCTAACGCCGCTTTTTTTGTTT CTGGTCTCCCTTGCTTGTACTTTACAGTATAGCTTCCTCAATGATCTCCTTATCCGGTTAAGATGGCAAGCTTGACAAGTATTTCT TCAGAATTTACAATGAAGTTTACTAGAGGATTAACTAATAAGGAGGGCAAACTACTAGAGATGTCTAAACGTCGTATGAAGTA CCACAGCAACAATGAGATCAGCTATTACAATTTCCTACATAGCATGAAGGACAAAATCGTGACGGTGTATAGAGGCGGCCCTG AGAGCAAGAAGGGCAAGCTGACGGCAGTGAAGAGCGACTACATCGCGCTGCAGGCGGAGAAGAAGATCATCTACTATCAAC TTGAACACGTTAAGTCAATCACGGAAGACACAAACAACTCAACAACGACGATCGAAACGGAAGAGATGTTAGACGCGGACGA CTTCCACTCACTGATCGGCCACCTGATCAATCAGAGCGTGCAGTTCAATCAGGGAGGACCAGAAAGCAAGAAAGGCCGCCTTG TATGGTTAGGCGACGACTATGCGGCACTGAATACGAACGAAGACGGAGTGGTATACTTCAACATTCACCACATTAAGTCCATC TCAAAGCACGAACCGGACCTTAAGATCGAGGAACAAACACCGGTGGGCGTGCTTGAGGCGGACGACCTGTCAGAAGTGTTCA AATCATTAACGCACAAGTGGGTGAGCATCAACAGAGGCGGCCCTGAGGCAATCGAAGGCATTTTAGTGGACAACGCAGATGG ACACTACACGATCGTTAAGAACCAGGAAGTATTGAGAATTTACCCGTTCCACATCAAATCAATTTCACTGGGCCCGAAGGGAA GTTATAAAAAGGAAGACCAGAAGAACGAGCAGAATCAAGAGGATAACAACGACAAAGATTCAAACAGCTTTATCAGCAGCAA AAGCTATTCAAGCAGTAAAAGCTCAAAGCGGAGCTTGAAGAGCTCTGACGACCAGAGCAGTAAGTCAGGCAGAAGTTCCAGA AGCAAGAGTAGCTCCAAGAGTAGTAAGAGGAGTTTGAAGTCCAGCGACTACCAGAGCAGTAAAAGTGGAAGAAGTTCCAGG AGCAAGTCCTCAAGTAAGTCCAGTAAGAGAAGCCTGAAGAGTAGCGACTACCAGAGCAGCAAAAGCTCAAAAAGGAGCCCG CGCAGTAGCGACTACCAGAGTAGTCGTAGCCCGGGATATAGCTCCTCCATCAAGTCTAGTGGCAAGCAGAAAGAGGACTATTC ATACGAGACAATCGTTCGCACAATCGATTATCATTGGAAGAGGAAATTCGGTTTCAAACTCGGGGGGGACGGAGGTGAAATG TCTAAGGGCGAAGAGTTATTCACGGGCGTAGTTCCGATTCTTGTGGAGCTCGACGGTGATGTAAACGGGCACAAGTTTTCTGT TTCTGGGGAAGGTGAAGGCGACGCGACCTATGGGAAACTCACTCTTAAGTTCATCTGCACCACGGGGAAGTTACCAGTCCCGT GGCCAACACTGGTGACTACCTTCGCATACGGACTGCAGTGTTTTGCGCGTTATCCTGATCACATGAAACAACACGATTTTTTCA AGAGCGCCATGCCAGAAGGGTACGTCCAAGAACGCACGATTTTTTTCAAAGATGATGGGAATTACAAAACGCGTGCAGAAGT AAAATTTGAGGGCGACACCCTGGTAAACCGTATTGAACTCAAAGGAATCGACTTTAAGGAGGACGGAAACATTCTGGGCCAC AAACTGGAATATAACTACAACTCACATAACGTTTATATAATGGCTGATAAGCAAAAAAATGGTATCAAGGTGAACTTTAAGATA AGACATAACATTGAGGACGGCAGTGTCCAACTTGCAGACCATTACCAGCAGAACACACCAATTGGCGACGGTCCAGTACTTTT ACCGGACAACCACTATCTCTCAACTCAGAGTGCTCTGTCAAAGGACCCTAATGAAAAACGCGACCACATGGTACTGCTCGAGTT TGTGACTGCGGCCGGAATCACCCACGGGATGGACGAGCTTTACAAGTAATCAAATGTCAGACGAAAATGCCAATTATTGAAGC GGCTAACGCCGCTTTTTTTGTTTCTGGTCTCCCAACTTTGCTAAAGAGCGTCTCAGCTTCTAGAG

>pCas9g2-D15-GFP

TAGAGATCTGCAGTGACTGGAGCTTCTAGAGATCAGGTCAGACGCGATTTCCTGGGTGCTCGAGCCATGGGAAAGCGGTGGT GGAAAAAACGCCGAATGAAGCGGATGAAATTGCTCATCGTCTCGTGAAAAAGCCGAAGAAGGTTAAGCCTGGGTATAAAAAG AAAATGAGCTATGAGATGGAGAAATTGAAGTCATGCGCCGGTTAGTTTTAGAGCTAGAAATAGCAAGTTAAAATAAGGCTAG TCCGTTATCAACTTGAAAAAGTGGCACCGAGTCGGTGCTTTTTTTCTCGGTACCAAATTCCAGAAAAGAGGCCTCCCGAAAGG GGGGCCTTTTTTCGTTTTGGTCCCTCAATGATCCGACAGGAAGTGGTATTAGCGACTCCTACAAGGAATAGGTGTCTGGGTCAT GCAGTGACTGGGAAAACCCTGGCGACTAGTCTTGGACTCCTGTTGATAGATCCAGTAATGACCTCAGAACTCCATCTGGATTTG TTCAGAACGCTCGGTTGCCGCCGGGCGTTTTTTATTGGTGAGAATCCAGGGGTCCCCAATAATTACGATTTAAATCCTTCAAAC TTCCCAAAGGCGAGCCCTAGTGACATTAGAAAACCGACTGTAAAAAGTACAGTCGGCATTATCTCATATTATAAAAGCCAGTCA TTAGGCCTATCTGACAATTCCTGAATAGAGTTCATAAACAATCCTGCATGATAACCATCACAAACAGAATGATGTACCTGTAAA GATAGCGGTAAATATATTGAATTACCTTTATTAATGAATTTTCCTGCTGTAATAATGGGTAGAAGGTAATTACTATTATTATTGA TATTTAAGTTAAACCCAGTAAATGAAGTCCATGGAATAATAGAAAGAGAAAAAGCATTTTCAGGTATAGGTGTTTTGGGAAAC AATTTCCCCGAACCATTATATTTCTCTACATCAGAAAGGTATAAATCATAAAACTCTTTGAAGTCATTCTTTACAGGAGTCCAAA TACCAGAGAATGTTTTAGATACACCATCAAAAATTGTATAAAGTGGCTCTAACTTATCCCAATAACCTAACTCTCCGTCGCTATT GTAACCAGTTCTAAAAGCTGTATTTGAGTTTATCACCCTTGTCACTAAGAAAATAAATGCAGGGTAAAATTTATATCCTTCTTGT TTTATGTTTCGGTATAAAACACTAATATCAATTTCTGTGGTTATACTAAAAGTCGTTTGTTGGTTCAAATAATGATTAAATATCTC TTTTCTCTTCCAATTGTCTAAATCAATTTTATTAAAGTTCATTTGATATGCCTCCTAAATTTTTATCTAAAGTGAATTTAGGAGGCT TACTTGTCTGCTTTCTTCATTAGAATCAATCCTTTTTTAAAAGTCAATCCCGTTTGTTGAAGACTTTTGTCCTTTTCCGCTGCATAA ACAATATGGCCCGTTTGTTGAACTACTCTTTAATAAAATAATTTTTCCGTTCCCAATTCCACATTGCAATAATAGAAAATCCATCT TCATCGGCTTTTTCGTCATCATCTGTATGAATCAAATCGCCTTCTTCTGTGTCATCAAGGTTTAATTTTTTATGTATTTCTTTTAAC AAACCACCATAGGAGATTAACCTTTTACGGTGTAAACCTTCCTCCAAATCAGACAAACGTTTCAAATTCTTTTCTTCATCATCGG TCATAAAATCCGTATCCTTTACAGGATATTTTGCAGTTTCGTCAATTGCCGATTGTATATCCGATTTATATTTATTTTTCGGTCGA ATCATTTGAACTTTTACATTTGGATCATAGTCTAATTTCATTGCCTTTTTCCAAAATTGAATCCATTGTTTTTGATTCACGTAGTTT TCTGTATTCTTAAAATAAGTTGGTTCCACACATACCAATACATGCATGTGCTGATTATAAGAATTATCTTTATTATTTATTGTCAC TTCCGTTGCACGCATAAAACCAACAAGATTTTTATTAATTTTTTTATATTGCATCATTCGGCGAAATCCTTGAGCCATATCTGACA 
AACTCTTATTTAATTCTTCGCCATCATAAACATTTTTAACTGTTAATGTGAGAAACAACCAACGAACTGTTGGCTTTTGTTTAATA ACTTCAGCAACAACCTTTTGTGACTGAATGCCATGTTTCATTGCTCTCCTCCAGTTGCACATTGGACAAAGCCTGGATTTACAAA ACCACACTCGATACAACTTTCTTTCGCCTGTTTCACGATTTTGTTTATACTCTAATATTTCAGCACAATCTTTTACTCTTTCAGCCT TTTTAAATTCAAGAATATGCAGAAGTTCAAAGTAATCAACATTAGCGATTTTCTTTTCTCTCCATGGTCTCACTTTTCCACTTTTT GTCTTGTCCACTAAAACCCTTGATTTTTCATCTGAATAAATGCTACTATTAGGACACATAATATTAAAAGAAACCCCCATCTATTT AGTTATTTGTTTGGTCACTTATAACTTTAACAGATGGGGTTTTTCTGTGCAACCAATTTTAAGGGTTTTCCAATACTTTAAAACAC ATACATACCAACACTTCAACGCACCTTTCAGCAACTAAAATAAAAATGACGTTATTTCTATATGTATCAAGATAAGAAAGAACA AGTTCAAAACCATCAAAAAAAGACACCTTTTCAGGTGCTTTTTTTTATTTTATAAACTCATTCCCTGATCTCGACTTCGTTCTTTTTT TACCTCTCGGTTATGAGTTAGTTCAAATTCGTTCTTTTTAGGTTCTAAATCGTGTTTTTCTTGGAATTGTGCTGTTTTATCCTTTAC CTTGTCTACAAACCCCTTAAAAACGTTTTTAAAGGCTTTTAAGCGTCTGTACGTTCCTTAAGGAATTATTCCTTAGTGCTTTCTAG GTTAATGTCATGATAATAATGGTTTCTTAGACGTCAGGTGGCACTTTTCGGGGAAATGTCCGCGGAACCCCTATTTGTTTATTTT TCTAAATACATTCAAATATGTATCCGCTCATGAGACAATAACCCTGATAAATGCTTCAATAATATTGAAAAAGGAAGAGTATGA GTATTCAACATTTCCGTGTCGCCCTTATTCCCTTTTTTGCGGCATTTTTGCCTTCCTGTTTTTTGCTCACCCAGAAACGCTGGTGAAA GTAAAAGATGCTGAAGATCAGTTGGGTGCACGAGTGGGTTACATCGAACTGGATCTCAACAGCGGTAAGATCCTTGAGAGTT TTCGCCCCGAAGAACGTTTTCCAATGATGAGCACTTTTAAAGTTCTGCTATGTGGCGCGGTATTATCCCGTGTTGACGCCGGGC AAGAGCAACTCGGTCGCCGCATACACTATTCTCAGAATGACTTGGTTGAGTACTCACCAGTCACAGAAAAGCATCTTACGGAT GGCATGACAGTAAGAGAATTATGCAGTGCTGCCATAACCATGAGTGATAACACTGCGGCCAACTTACTTCTGACAACGATCGG AGGACCGAAGGAGCTAACCGCTTTTTTGCACAACATGGGGGATCATGTAACTCGCCTTGATCGTTGGGAACCGGAGCTGAATG AAGCCATACCAAACGACGAGCGTGACACCACGATGCCTGCAGCAATGGCAACAACGTTGCGCAAACTATTAACTGGCGAACTA CTTACTCTAGCTTCCCGGCAACAATTAATAGACTGGATGGAGGCGGATAAAGTTGCAGGACCACTTCTGCGCTCGGCCCTTCCG GCTGGCTGGTTTATTGCTGATAAATCTGGAGCCGGTGAGCGTGGGTCTCGCGGTATCATTGCAGCACTGGGGCCAGATGGTA AGCCCTCCCGTATCGTAGTTATCTACACGACGGGGAGTCAGGCAACTATGGATGAACGAAATAGACAGATCGCTGAGATAGG TGCCTCACTGATTAAGCATTGGTAACTGTCAGACCAAGTTTACTCATATATACTTTAGATTGATTTAAAACTTCATTTTTAATTTA AAAGGATCTAGGTGAAGATCCTTTTTGATAATCTCATGACCAAAATCCCTTAACGTGAGTTTTCGTTCCACTGAGCGTCAGACC CCTTAATAAGATGATCTTCTTGAGATCGTTTTGGTCTGCGCGTAATCTCTTGCTCTGAAAACGAAAAAACCGCCTTGCAGGGAG GTTTTTCGAAGGTTCTCTGAGCTACCAACTCTTTGAACCGAGGTAACTGGCTTGCAGGAGCGCAGTCACCAAAACTTGTCCTTT CAGTTTAGCCTTAACCGGCGCATGACTTCAAGACTAACTCCTCTAAATCAATTACCAGTGGCTGCTGCCAGTGGTGCTTTTGCAT GTCTTTCCGGGTTGGACTCAAGACGATAGTTACCGGATAAGGCGCAGCGGTCGGACTGAACGGGGGGTTCGTGCATACAGTC CAGCTTGGAGCGAACTGCCTACCCGGAACTGAGTGTCAGGCGTGGAATGAGACAAACGCGGCCATAACAGCGGAATGACACC GGTAAACCGAAAGGCAGGAACAGGAGAGCGCACGAGGGAGCCGCCAGGGGGAAACGCCTGGTATCTTTATAGTCCTGTCGG GTTTCGCCACCACTGATTTGAGCGTCAGATTTCGTGATGCTTGTCAGGGGGCGGAGCCTATGGAAAAACGCTTTGCCCGAGCC TATGGAAAAACGCTTTGCCCCACACAGGCTCTTGAACAAGGGATAACAATTTGTCATAGCTGTATTCGTTACAAGGAGGATTTT CGAAAAAACACCCTAACGGGTGTTTTTTTATAGCTGGTCTCCCCAAGTGCTGTAAAAAACGCCGAATGAAGCGGATGAAATTG CTCATCGTCTCGTGAAAAAGCCGAAGAAGGTTAAGCCTGGGTATAAAAAGAAAATGAGCTATGAGATGGAGAAAGCCATAAC CATGAGTGATAACACTGGTTTTAGAGCTAGAAATAGCAAGTTAAAATAAGGCTAGTCCGTTATCAACTTGAAAAAGTGGCACC GAGTCGGTGCTTTTTTTCTCGGTACCAAATTCCAGAAAAGAGGCCTCCCGAAAGGGGGGCCTTTTTTCGTTTTGGTCCCTCAAT GATCTCCTTATCCGGTTAAGATGGCTTTTAAATCAATTTTCAGCTCCTGTATACAATTACCAAAGTTTTTCTGAATGAAGCCATGT GTTTTGACACATTCTATACTCACAAGGAGGTGAGACACATGAGTAAATCCTGGGGCAAATTCATCGAGGAGGAAGAGGCGGA AATGGCTTCCCGTCGTAATCTAATGATTGTCGATGGAACTAACTTAGGCTTTCGCTTCAAACATAACAATAGTAAAAAACCATTT GCCTCAAGTTATGTTTCAACTATTCAATCTCTGGCAAAATCCTACTCTGCCAGAACTACGATTGTTCTAGGTGATAAGGGCAAA AGCGTGTTCAGACTAGAACATCTACCAGAGTATAAAGGTAATCGTGATGAAAAGTACGCACAACGTACGGAAGAGGAGAAAG CGCTAGATGAGCAGTTCTTCGAATACCTCAAAGATGCTTTCGAGTTGTGTAAAACTACATTCCCAACTTTTACCATTCGTGGTGT AGAAGCAGATGACATGGCGGCGTATATTGTTAAGCTCATCGGGCATCTTTATGATCACGTTTGGCTAATATCTACAGATGGTG ACTGGGATACTTTATTAACGGATAAAGTTTCTCGTTTTTCTTTCACAACACGTCGTGAGTATCATCTTCGTGATATGTATGAGCA CCATAACGTGGACGACGTAGAACAGTTTATCTCCCTGAAAGCAATTATGGGAGATCTAGGAGATAATATTCGTGGTGTTGAAG GAATAGGAGCAAAACGCGGATATAATATTATTCGTGAGTTTGGTAACGTACTGGATATTATTGATCAGCTTCCACTGCCTGGAA AGCAGAAATATATACAGAACCTGAATGCATCGGAAGAACTGCTTTTCCGAAACTTGATTCTGGTTGATTTACCTACCTACTGTG TGGATGCTATTGCTGCTGTAGGTCAAGATGTGTTAGATAAGTTCACGAAAGACATCCTTGAGATCGCGGAACAATGATAAGCT CTTAAAGGGGGTTTTAGATACCAGAGATGGATAAGAAATACTCAATAGGCTTAGATATCGGCACAAATAGCGTCGGATGGGC GGTGATCACTGATGAATATAAGGTTCCGTCTAAAAAGTTCAAGGTTCTGGGAAATACAGACCGCCACAGTATCAAAAAAAATC TTATAGGGGCTCTTTTATTTGACAGTGGAGAGACAGCGGAAGCGACTCGTCTCAAACGGACAGCTCGTAGAAGGTATACACGT CGGAAGAATCGTATTTGTTATCTACAGGAGATTTTTTCAAATGAGATGGCGAAAGTAGATGATAGTTTCTTTCATCGACTTGAA GAGTCTTTTTTGGTGGAAGAAGACAAGAAGCATGAACGTCATCCTATTTTTGGAAATATAGTAGATGAAGTTGCTTATCATGA 
GAAATATCCAACTATCTATCATCTGCGAAAAAAATTGGTAGATTCTACTGATAAAGCGGATTTGCGCTTAATCTATTTGGCCTTA GCGCATATGATTAAGTTTCGTGGTCATTTTTTGATTGAGGGAGATTTAAATCCTGATAATAGTGATGTGGACAAACTATTTATCC AGTTGGTACAAACCTACAATCAATTATTTGAAGAAAACCCTATTAACGCAAGTGGAGTAGATGCTAAAGCGATTCTTTCTGCAC GATTGAGTAAATCAAGACGATTAGAAAATCTCATTGCTCAGCTCCCCGGTGAGAAGAAAAATGGCTTATTTGGGAATCTCATT GCTTTGTCATTGGGTTTGACCCCTAATTTTAAATCAAATTTTGATTTGGCAGAAGATGCTAAATTACAGCTTTCAAAAGATACTT ACGATGATGATTTAGATAATTTATTGGCGCAAATTGGAGATCAATATGCTGATTTGTTTTTGGCAGCTAAGAATTTATCAGATG CTATTTTACTTTCAGATATCCTAAGAGTAAATACTGAAATAACTAAGGCTCCCCTATCAGCTTCAATGATTAAACGCTACGATGA ACATCATCAAGACTTGACTCTTTTAAAAGCTTTAGTTCGACAACAACTTCCAGAAAAGTATAAAGAAATCTTTTTTGATCAATCA AAAAACGGATATGCAGGTTATATTGATGGGGGAGCTAGCCAAGAAGAATTTTATAAATTTATCAAACCAATTTTAGAAAAAAT GGATGGTACTGAGGAATTATTGGTGAAACTAAATCGTGAAGATTTGCTGCGCAAGCAACGGACCTTTGACAACGGCTCTATTC CCCATCAAATTCACTTGGGTGAGCTGCATGCTATTTTGAGAAGACAAGAAGACTTTTATCCATTTTTAAAAGACAATCGTGAGA AGATTGAAAAAATCTTGACTTTTCGAATTCCTTATTATGTTGGTCCATTGGCGCGTGGCAATAGTCGTTTTGCATGGATGACTCG GAAGTCTGAAGAAACAATTACCCCATGGAATTTTGAAGAAGTTGTCGATAAAGGTGCTTCAGCTCAATCATTTATTGAACGCAT GACAAACTTTGATAAAAATCTTCCAAATGAAAAAGTACTACCAAAACATAGTTTGCTTTATGAGTATTTTACGGTTTATAACGAA TTGACAAAGGTCAAATATGTTACTGAAGGAATGCGAAAACCAGCATTTCTTTCAGGTGAACAGAAGAAAGCCATTGTTGATTT ACTCTTCAAAACAAATCGAAAAGTAACCGTTAAGCAATTAAAAGAAGATTATTTCAAAAAAATAGAATGTTTTGATAGTGTTGA AATTTCAGGAGTTGAAGATAGATTTAATGCTTCATTAGGTACCTACCATGATTTGCTAAAAATTATTAAAGATAAAGATTTTTTG GATAATGAAGAAAATGAAGATATCTTAGAGGATATTGTTTTAACATTGACCTTATTTGAAGATAGGGAGATGATTGAGGAAAG ACTTAAAACATATGCTCACCTCTTTGATGATAAGGTGATGAAACAGCTTAAACGTCGCCGTTATACTGGTTGGGGACGTTTGTC TCGAAAATTGATTAATGGTATTAGGGATAAGCAATCTGGCAAAACAATATTAGATTTTTTGAAATCAGATGGTTTTGCCAATCG CAATTTTATGCAGCTGATCCATGATGATAGTTTGACATTTAAAGAAGACATTCAAAAAGCACAAGTGTCTGGACAAGGCGATA GTTTACATGAACATATTGCAAATTTAGCTGGTAGCCCTGCTATTAAAAAAGGTATTTTACAGACTGTAAAAGTTGTTGATGAAT TGGTCAAAGTAATGGGGCGGCATAAGCCAGAAAATATCGTTATTGAAATGGCACGTGAAAATCAGACAACTCAAAAGGGCCA GAAAAATTCGCGAGAGCGTATGAAACGAATCGAAGAAGGTATCAAAGAATTAGGAAGTCAGATTCTTAAAGAGCATCCTGTT GAAAATACTCAATTGCAAAATGAAAAGCTCTATCTCTATTATCTCCAAAATGGAAGAGACATGTATGTGGACCAAGAATTAGAT ATTAATCGTTTAAGTGATTATGATGTCGATCACATTGTTCCACAAAGTTTCCTTAAAGACGATTCAATAGACAATAAGGTCTTAA CGCGTTCTGATAAAAATCGTGGTAAATCGGATAACGTTCCAAGTGAAGAAGTAGTCAAAAAGATGAAAAACTATTGGAGACA ACTTCTAAACGCCAAGTTAATCACTCAACGTAAGTTTGATAATTTAACGAAAGCTGAACGTGGAGGTTTGAGTGAACTTGATAA AGCTGGTTTTATCAAACGCCAATTGGTTGAAACTCGCCAAATCACTAAGCATGTGGCACAAATTTTGGATAGTCGCATGAATAC TAAATACGATGAAAATGATAAACTTATTCGAGAGGTTAAAGTGATTACCTTAAAATCTAAATTAGTTTCTGACTTCCGAAAAGA TTTCCAATTCTATAAAGTACGTGAGATTAACAATTACCATCATGCCCATGATGCGTATCTAAATGCCGTCGTTGGAACTGCTTTG ATTAAGAAATATCCAAAACTTGAATCGGAGTTTGTCTATGGTGATTATAAAGTTTATGATGTTCGTAAAATGATTGCTAAGTCT GAGCAAGAAATAGGCAAAGCAACCGCAAAATATTTCTTTTTACTCTAATATCATGAACTTCTTCAAAACAGAAATTACACTTGCA AATGGAGAGATTCGCAAACGCCCTCTAATCGAAACTAATGGGGAAACTGGAGAAATTGTCTGGGATAAAGGGCGAGATTTTG CCACAGTGCGCAAAGTATTGTCCATGCCCCAAGTCAATATTGTCAAGAAAACAGAAGTACAGACAGGCGGATTCTCCAAGGAG TCAATTTTACCAAAAAGAAATTCGGACAAGCTTATTGCTCGTAAAAAAGACTGGGATCCAAAAAAATATGGTGGTTTTGATAGT CCAACGGTAGCTTATTCAGTCCTAGTGGTTGCTAAGGTGGAAAAAGGGAAATCGAAGAAGTTAAAATCCGTTAAAGAGTTACT AGGGATCACAATTATGGAAAGAAGTTCCTTTGAAAAAAATCCGATTGACTTTTTAGAAGCTAAAGGATATAAGGAAGTTAAAA AAGACTTAATCATTAAACTACCTAAATATAGTCTTTTTTGAGTTAGAAAACGGTCGTAAACGGATGCTGGCTAGTGCCGGAGAAT TACAAAAAGGAAATGAGCTGGCTCTGCCAAGCAAATATGTGAATTTTTTATATTTAGCTAGTCATTATGAAAAGTTGAAGGGTA GTCCAGAAGATAACGAACAAAAACAATTGTTTGTGGAGCAGCATAAGCATTATTTAGATGAGATTATTGAGCAAATCAGTGAA TTTTCTAAGCGTGTTATTTTAGCAGATGCCAATTTAGATAAAGTTCTTAGTGCATATAACAAACATAGAGACAAACCAATACGT GAACAAGCAGAAAATATTATTCATTTATTTACGTTGACGAATCTTGGAGCTCCCGCTGCTTTTAAATATTTTGATACAACAATTG ATCGTAAACGATATACGTCTACAAAAGAAGTTTTAGATGCCACTCTTATCCATCAATCCATCACTGGTCTTTATGAAACACGCAT TGATTTGAGTCAGCTAGGAGGTGACTGATAATCAAATGTCAGACGAAAATGCCAATTATTGAAGCGGCTAACGCCGCTTTTTTT GTTTCTGGTCTCCCTTGCTTGTACTTTACAGTATAGCTTCCTCAATGATCTCCTTATCCGGTTAAGATGGCAAGCTTGACAAGTAT TTCTTCAGAATTTACAATGAAGTTTACTAGAGGATTAACTAATAAGGAGGGCAAACTACTAGAGATGTCTAAACGTCGTATGA AGTACCACAGCAACAATGAGATCAGCTATTACAATTTCCTACATAGCATGAAGGACAAAATCGTGACGGTGTATAGAGGCGGC CCTGAGAGCAAGAAGGGCAAGCTGACGGCAGTGAAGAGCGACTACATCGCGCTGCAGGCGGAGAAGAAGATCATCTACTAT CAACTTGAACACGTTAAGTCAATCACGGAAGACACAAACAACTCAACAACGACGATCGAAACGGAAGAGATGTTAGACGCGG ACGACTTCCACTCACTGATCGGCCACCTGATCAATCAGAGCGTGCAGTTCAATCAGGGAGGACCAGAAAGCAAGAAAGGCCG CCTTGTATGGTTAGGCGACGACTATGCGGCACTGAATACGAACGAAGACGGAGTGGTATACTTCAACATTCACCACATTAAGT CCATCTCAAAGCACGAACCGGACCTTAAGATCGAGGAACAAACACCGGTGGGCGTGCTTGAGGCGGACGACCTGTCAGAAGT 
GTTCAAATCATTAACGCACAAGTGGGTGAGCATCAACAGAGGCGGCCCTGAGGCAATCGAAGGCATTTTAGTGGACAACGCA GATGGACACTACACGATCGTTAAGAACCAGGAAGTATTGAGAATTTACCCGTTCCACATCAAATCAATTTCACTGGGCCCGAA GGGAAGTTATAAAAAGGAAGACCAGAAGAACGAGCAGAATCAAGAGGATAACAACGACAAAGATTCAAACAGCTTTATCAG CAGCAAAAGCTATTCAAGCAGTAAAAGCTCAAAGCGGAGCTTGAAGAGCTCTGACGACCAGAGCAGTAAGTCAGGCAGAAGT TCCAGAAGCAAGAGTAGCTCCAAGAGTAGTAAGAGGAGTTTGAAGTCCAGCGACTACCAGAGCAGTAAAAGTGGAAGAAGT TCCAGGAGCAAGTCCTCAAGTAAGTCCAGTAAGAGAAGCCTGAAGAGTAGCGACTACCAGAGCAGCAAAAGCTCAAAAAGG AGCCCGCGCAGTAGCGACTACCAGAGTAGTCGTAGCCCGGGATATAGCTCCTCCATCAAGTCTAGTGGCAAGCAGAAAGAGG ACTATTCATACGAGACAATCGTTCGCACAATCGATTATCATTGGAAGAGGAAATTCGGTTTCAAACTCGGGGGGGACGGAGGT GAAATGTCTAAGGGCGAAGAGTTATTCACGGGCGTAGTTCCGATTCTTGTGGAGCTCGACGGTGATGTAAACGGGCACAAGT TTTCTGTTTCTGGGGAAGGTGAAGGCGACGCGACCTATGGGAAACTCACTCTTAAGTTCATCTGCACCACGGGGAAGTTACCA GTCCCGTGGCCAACACTGGTGACTACCTTCGCATACGGACTGCAGTGTTTTGCGCGTTATCCTGATCACATGAAACAACACGAT TTTTTCAAGAGCGCCATGCCAGAAGGGTACGTCCAAGAACGCACGATTTTTTTCAAAGATGATGGGAATTACAAAACGCGTGC AGAAGTAAAATTTGAGGGCGACACCCTGGTAAACCGTATTGAACTCAAAGGAATCGACTTTAAGGAGGACGGAAACATTCTG GGCCACAAACTGGAATATAACTACAACTCACATAACGTTTATATAATGGCTGATAAGCAAAAAAATGGTATCAAGGTGAACTTT AAGATAAGACATAACATTGAGGACGGCAGTGTCCAACTTGCAGACCATTACCAGCAGAACACACCAATTGGCGACGGTCCAGT ACTTTTACCGGACAACCACTATCTCTCAACTCAGAGTGCTCTGTCAAAGGACCCTAATGAAAAACGCGACCACATGGTACTGCT CGAGTTTGTGACTGCGGCCGGAATCACCCACGGGATGGACGAGCTTTACAAGTAATCAAATGTCAGACGAAAATGCCAATTAT TGAAGCGGCTAACGCCGCTTTTTTTGTTTCTGGTCTCCCAACTTTGCTAAAGAGCGTCTCAGCTTCTAGAG

>pCas9g1-D15-KinA

ttaagatggcaagcttgacagccgaattcggattcccgcgTATATCTTGAATTAAAATTAAAATCTGCCAAGATCGAAAAATAAAAAGGATTT TTTGTGTCATTGGCGAATTATGATCTATTGAAGCAACCGTTAAGCTTACATAAGGAGGAACTACTATGGAACAGGATACGCAG CATGTTAAACCACTTCAAACAAAAACCGATATTCATGCAGTCTTGGCCTCTAATGGACGCATCATTTATATATCTGCCAACTCCA AACTGCATTTGGGCTATCTCCAAGGAGAGATGATCGGATCATTCCTCAAAACGTTTCTGCATGAGGAAGACCAATTTTTGGTTG AAAGCTATTTTTATAATGAACATCATCTGATGCCGTGCACCTTTCGTTTTATTAAAAAAGATCATACGATTGTGTGGGTGGAGG CTGCGGTAGAAATTGTTACGACAAGAGCTGAGCGGACAGAACGGGAAATCATTTTGAAAATGAAGGTTCTTGAAGAAGAAAC AGGCCATCAATCCCTAAACTGCGAAAAACATGAAATCGAACCTGCAAGCCCGGAATCGACTACATATATAACGGATGATTATG AACGGTTGGTTGAAAATCTCCCGAGTCCGCTATGCATCAGTGTCAAAGGCAAGATCGTCTATGTAAACAGCGCGATGCTTTCA ATGCTGGGAGCCAAAAGCAAGGATGCTATTATTGGTAAATCGTCCTATGAATTTATTGAAGAAGAATATCATGATATCGTGAA AAACAGGATTATACGAATGCAAAAAGGAATGGAAGTCGGAATGATTGAACAGACGTGGAAAAGGCTTGATGGCACACCTGTT CATTTAGAAGTGAAAGCATCCCCGACCGTCTACAAAAACCAGCAGGCTGAGCTGCTGCTGCTGATCGATATCTCTTCAAGGAA AAAATTCCAAACCATCCTGCAAAAAAGCCGTGAACGATATCAGCTGCTGATTCAAAATTCCATTGATACCATTGCGGTGATTCA CAATGGAAAATGGGTATTTATGAATGAATCGGGAATTTCCCTGTTTGAAGCGGCTACATATGAGGATTTAATTGGCAAAAACA TATACGATCAGCTGCATCCTTGCGATCACGAGGATGTAAAAGAGAGAATCCAAAACATTGCCGAGCAAAAAACAGAATCTGAA ATTGTCAAGCAATCCTGGTTCACCTTTCAGAACAGGGTCATCTATACGGAGATGGTCTGCATTCCGACGACCTTTTTTGGTGAA GCGGCCGTCCAGGTCATTCTTCGGGACATCTCAGAGAGAAAACAAACAGAAGAATTGATGCTGAAATCGGAAAAATTATCAAT CGCAGGGCAGCTCGCGGCGGGAATCGCCCATGAGATCCGCAACCCTCTTACAGCGATCAAAGGATTTTTACAGCTGATGAAAC CGACAATGGAAGGCAACGAACATTACTTTGATATTGTGTTTTCTGAACTCAGCCGTATCGAATTAATACTCAGTGAACTGCTCA TGCTGGCGAAACCTCAGCAAAATGCTGTCAAAGAATATTTGAACTTGAAAAAATTAATTGGTGAGGTTTCAGCCCTGTTAGAA ACGCAGGCGAATTTAAATGGCATTTTTATCAGAACAAGTTATGAAAAAGACAGCATTTATATAAACGGGGATCAAAACCAATT AAAGCAGGTATTCATTAATTTAATCAAAAATGCAGTTGAATCAATGCCTGATGGGGGAACAGTAGACATTATCATAACCGAAG ATGAGCATTCTGTTCATGTTACTGTCAAAGACGAAGGGGAAGGTATACCTGAAAAGGTACTAAACCGGATTGGAGAGCCATTT TTAACAACAAAAGAAAAAGGTACGGGGCTTGGATTAATGGTGACATTTAATATCATTGAAAACCATCAGGGAGTTATACATGT GGACAGCCATCCTGAAAAAGGCACAGCGTTTAAAATTTCATTTCcaaaaaaataagcatgcccggggatggacgagctttacaaGTAATCAA ATGTCAGACGAAAATGCCAATTATTGAAGCGGCTAACGCCGCTTTTTTTGTTTCTGGTCTCCCAACTTTGCTAAAGAGCGTCTCA GCTTCTAGAGGTGACTGGGAAAACCCTGGCGACTAGTCTTGGACTCCTGTTGATAGATCCAGTAATGACCTCAGAACTCCATCT GGATTTGTTCAGAACGCTCGGTTGCCGCCGGGCGTTTTTTATTGGTGAGAATCCAGGGGTCCCCAATAATTACGATTTAAATCC TTCAAACTTCCCAAAGGCGAGCCCTAGTGACATTAGAAAACCGACTGTAAAAAGTACAGTCGGCATTATCTCATATTATAAAAG CCAGTCATTAGGCCTATCTGACAATTCCTGAATAGAGTTCATAAACAATCCTGCATGATAACCATCACAAACAGAATGATGTAC CTGTAAAGATAGCGGTAAATATATTGAATTACCTTTATTAATGAATTTTCCTGCTGTAATAATGGGTAGAAGGTAATTACTATTA TTATTGATATTTAAGTTAAACCCAGTAAATGAAGTCCATGGAATAATAGAAAGAGAAAAAGCATTTTCAGGTATAGGTGTTTTG GGAAACAATTTCCCCGAACCATTATATTTCTCTACATCAGAAAGGTATAAATCATAAAACTCTTTGAAGTCATTCTTTACAGGAG TCCAAATACCAGAGAATGTTTTAGATACACCATCAAAAATTGTATAAAGTGGCTCTAACTTATCCCAATAACCTAACTCTCCGTC 
GCTATTGTAACCAGTTCTAAAAGCTGTATTTGAGTTTATCACCCTTGTCACTAAGAAAATAAATGCAGGGTAAAATTTATATCCT TCTTGTTTTATGTTTCGGTATAAAACACTAATATCAATTTCTGTGGTTATACTAAAAGTCGTTTGTTGGTTCAAATAATGATTAAA TATCTCTTTTCTCTTCCAATTGTCTAAATCAATTTTATTAAAGTTCATTTGATATGCCTCCTAAATTTTTATCTAAAGTGAATTTAG GAGGCTTACTTGTCTGCTTTCTTCATTAGAATCAATCCTTTTTTAAAAGTCAATCCCGTTTGTTGAAGACTTTTGTCCTTTTCCGCT GCATAAACAATATGGCCCGTTTGTTGAACTACTCTTTAATAAAATAATTTTTCCGTTCCCAATTCCACATTGCAATAATAGAAAA TCCATCTTCATCGGCTTTTTCGTCATCATCTGTATGAATCAAATCGCCTTCTTCTGTGTCATCAAGGTTTAATTTTTTATGTATTTC TTTTAACAAACCACCATAGGAGATTAACCTTTTACGGTGTAAACCTTCCTCCAAATCAGACAAACGTTTCAAATTCTTTTCTTCAT CATCGGTCATAAAATCCGTATCCTTTACAGGATATTTTGCAGTTTCGTCAATTGCCGATTGTATATCCGATTTATATTTATTTTTC GGTCGAATCATTTGAACTTTTACATTTGGATCATAGTCTAATTTCATTGCCTTTTTTCCAAAATTGAATCCATTGTTTTTGATTCAC GTAGTTTTCTGTATTCTTAAAATAAGTTGGTTCCACACATACCAATACATGCATGTGCTGATTATAAGAATTATCTTTATTATTTA TTGTCACTTCCGTTGCACGCATAAAACCAACAAGATTTTTATTAATTTTTTTATATTGCATCATTCGGCGAAATCCTTGAGCCATA TCTGACAAACTCTTATTTAATTCTTCGCCATCATAAACATTTTTAACTGTTAATGTGAGAAACAACCAACGAACTGTTGGCTTTTG TTTAATAACTTCAGCAACAACCTTTTGTGACTGAATGCCATGTTTCATTGCTCTCCTCCAGTTGCACATTGGACAAAGCCTGGAT TTACAAAACCACACTCGATACAACTTTCTTTCGCCTGTTTCACGATTTTGTTTTATACTCTAATATTTCAGCACAATCTTTTACTCTT TCAGCCTTTTTAAATTCAAGAATATGCAGAAGTTCAAAGTAATCAACATTAGCGATTTTCTTTTCTCTCCATGGTCTCACTTTTCC ACTTTTTGTCTTGTCCACTAAAACCCTTGATTTTTCATCTGAATAAATGCTACTATTAGGACACATAATATTAAAAGAAACCCCCA TCTATTTAGTTATTTGTTTGGTCACTTATAACTTTAACAGATGGGGTTTTTCTGTGCAACCAATTTTAAGGGTTTTCCAATACTTT AAAACACATACATACCAACACTTCAACGCACCTTTCAGCAACTAAAATAAAAATGACGTTATTTCTATATGTATCAAGATAAGA AAGAACAAGTTCAAAACCATCAAAAAAAGACACCTTTTCAGGTGCTTTTTTTATTTTATAAACTCATTCCCTGATCTCGACTTCGT TCTTTTTTTACCTCTCGGTTATGAGTTAGTTCAAATTCGTTCTTTTTAAGGTTCTAAATCGTGTTTTTCTTGGAATTGTGCTGTTTTA TCCTTTACCTTGTCTACAAACCCCTTAAAAACGTTTTTAAAGGCTTTTAAGCGTCTGTACGTTCCTTAAGGAATTATTCCTTAGTG CTTTCTAGGTTAATGTCATGATAATAATGGTTTCTTAGACGTCAGGTGGCACTTTTCGGGGAAATGTCCGCGGAACCCCTATTT GTTTATTTTTCTAAATACATTCAAATATGTATCCGCTCATGAGACAATAACCCTGATAAATGCTTCAATAATATTGAAAAAGGAA GAGTATGAGTATTCAACATTTCCGTGTCGCCCTTATTCCCTTTTTTGCGGCATTTTGCCTTCCTGTTTTTGCTCACCCAGAAACGC TGGTGAAAGTAAAAGATGCTGAAGATCAGTTGGGTGCACGAGTGGGTTACATCGAACTGGATCTCAACAGCGGTAAGATCCT TGAGAGTTTTCGCCCCGAAGAACGTTTTCCAATGATGAGCACTTTTAAAGTTCTGCTATGTGGCGCGGTATTATCCCGTGTTGA CGCCGGGCAAGAGCAACTCGGTCGCCGCATACACTATTCTCAGAATGACTTGGTTGAGTACTCACCAGTCACAGAAAAGCATC TTACGGATGGCATGACAGTAAGAGAATTATGCAGTGCTGCCATAACCATGAGTGATAACACTGCGGCCAACTTACTTCTGACA ACGATCGGAGGACCGAAGGAGCTAACCGCTTTTTTGCACAACATGGGGGATCATGTAACTCGCCTTGATCGTTGGGAACCGG AGCTGAATGAAGCCATACCAAACGACGAGCGTGACACCACGATGCCTGCAGCAATGGCAACAACGTTGCGCAAACTATTAACT GGCGAACTACTTACTCTAGCTTCCCGGCAACAATTAATAGACTGGATGGAGGCGGATAAAGTTGCAGGACCACTTCTGCGCTC GGCCCTTCCGGCTGGCTGGTTTATTGCTGATAAATCTGGAGCCGGTGAGCGTGGGTCTCGCGGTATCATTGCAGCACTGGGGC CAGATGGTAAGCCCTCCCGTATCGTAGTTATCTACACGACGGGGAGTCAGGCAACTATGGATGAACGAAATAGACAGATCGCT GAGATAGGTGCCTCACTGATTAAGCATTGGTAACTGTCAGACCAAGTTTACTCATATATACTTTAGATTGATTTAAAACTTCATT TTTAATTTAAAAGGATCTAGGTGAAGATCCTTTTTGATAATCTCATGACCAAAATCCCTTAACGTGAGTTTTCGTTCCACTGAGC GTCAGACCCCTTAATAAGATGATCTTCTTGAGATCGTTTTGGTCTGCGCGTAATCTCTTGCTCTGAAAACGAAAAAACCGCCTTG CAGGGAGGTTTTTCGAAGGTTCTCTGAGCTACCAACTCTTTGAACCGAGGTAACTGGCTTGCAGGAGCGCAGTCACCAAAACT TGTCCTTTCAGTTTAGCCTTAACCGGCGCATGACTTCAAGACTAACTCCTCTAAATCAATTACCAGTGGCTGCTGCCAGTGGTGC TTTTGCATGTCTTTCCGGGTTGGACTCAAGACGATAGTTACCGGATAAGGCGCAGCGGTCGGACTGAACGGGGGGTTCGTGC ATACAGTCCAGCTTGGAGCGAACTGCCTACCCGGAACTGAGTGTCAGGCGTGGAATGAGACAAACGCGGCCATAACAGCGGA ATGACACCGGTAAACCGAAAGGCAGGAACAGGAGAGCGCACGAGGGAGCCGCCAGGGGGAAACGCCTGGTATCTTTATAGT CCTGTCGGGTTTCGCCACCACTGATTTGAGCGTCAGATTTCGTGATGCTTGTCAGGGGGCGGAGCCTATGGAAAAACGCTTTG CCCGAGCCTATGGAAAAACGCTTTGCCCCACACAGGCTCTTGAACAAGGGATAACAATTTGTCATAGCTGTATTCGTTACAAGG AGGATTTTCGAAAAAACACCCTAACGGGTGTTTTTTTATAGCTGGTCTCCCCAAGTGCTGTAAAAAACGCCGAATGAAGCGGA TGAAATTGCTCATCGTCTCGTGAAAAAGCCGAAGAAGGTTAAGCCTGGGTATAAAAAGAAAATGAGCTATGAGATGGAGAAA GCCATAACCATGAGTGATAACACTGGTTTTAGAGCTAGAAATAGCAAGTTAAAATAAGGCTAGTCCGTTATCAACTTGAAAAA GTGGCACCGAGTCGGTGCTTTTTTTCTCGGTACCAAATTCCAGAAAAGAGGCCTCCCGAAAGGGGGGCCTTTTTTCGTTTTGGT CCCTCAATGATCTCCTTATCCGGTTAAGATGGCTTTTAAATCAATTTTCAGCTCCTGTATACAATTACCAAAGTTTTTCTGAATGA AGCCATGTGTTTTGACACATTCTATACTCACAAGGAGGTGAGACACATGAGTAAATCCTGGGGCAAATTCATCGAGGAGGAAG AGGCGGAAATGGCTTCCCGTCGTAATCTAATGATTGTCGATGGAACTAACTTAGGCTTTCGCTTCAAACATAACAATAGTAAAA AACCATTTGCCTCAAGTTATGTTTCAACTATTCAATCTCTGGCAAAATCCTACTCTGCCAGAACTACGATTGTTCTAGGTGATAA GGGCAAAAGCGTGTTCAGACTAGAACATCTACCAGAGTATAAAGGTAATCGTGATGAAAAGTACGCACAACGTACGGAAGAG GAGAAAGCGCTAGATGAGCAGTTCTTCGAATACCTCAAAGATGCTTTCGAGTTGTGTAAAACTACATTCCCAACTTTTACCATT 
CGTGGTGTAGAAGCAGATGACATGGCGGCGTATATTGTTAAGCTCATCGGGCATCTTTATGATCACGTTTGGCTAATATCTACA GATGGTGACTGGGATACTTTATTAACGGATAAAGTTTCTCGTTTTTTCTTTCACAACACGTCGTGAGTATCATCTTCGTGATATGT ATGAGCACCATAACGTGGACGACGTAGAACAGTTTATCTCCCTGAAAGCAATTATGGGAGATCTAGGAGATAATATTCGTGGT GTTGAAGGAATAGGAGCAAAACGCGGATATAATATTATTCGTGAGTTTGGTAACGTACTGGATATTATTGATCAGCTTCCACT GCCTGGAAAGCAGAAATATATACAGAACCTGAATGCATCGGAAGAACTGCTTTTCCGAAACTTGATTCTGGTTGATTTACCTAC CTACTGTGTGGATGCTATTGCTGCTGTAGGTCAAGATGTGTTAGATAAGTTCACGAAAGACATCCTTGAGATCGCGGAACAAT GATAAGCTCTTAAAGGGGGTTTTAGATACCAGAGATGGATAAGAAATACTCAATAGGCTTAGATATCGGCACAAATAGCGTCG GATGGGCGGTGATCACTGATGAATATAAGGTTCCGTCTAAAAAGTTCAAGGTTCTGGGAAATACAGACCGCCACAGTATCAAA AAAAATCTTATAGGGGCTCTTTTATTTGACAGTGGAGAGACAGCGGAAGCGACTCGTCTCAAACGGACAGCTCGTAGAAGGT ATACACGTCGGAAGAATCGTATTTGTTATCTACAGGAGATTTTTTTCAAATGAGATGGCGAAAGTAGATGATAGTTTCTTTCATC GACTTGAAGAGTCTTTTTTGGTGGAAGAAGACAAGAAGCATGAACGTCATCCTATTTTTGGAAATATAGTAGATGAAGTTGCT TATCATGAGAAATATCCAACTATCTATCATCTGCGAAAAAAATTGGTAGATTCTACTGATAAAGCGGATTTGCGCTTAATCTATT TGGCCTTAGCGCATATGATTAAGTTTCGTGGTCATTTTTTGATTGAGGGAGATTTAAATCCTGATAATAGTGATGTGGACAAAC TATTTATCCAGTTGGTACAAACCTACAATCAATTATTTGAAGAAAACCCTATTAACGCAAGTGGAGTAGATGCTAAAGCGATTC TTTCTGCACGATTGAGTAAATCAAGACGATTAGAAAATCTCATTGCTCAGCTCCCCGGTGAGAAGAAAAATGGCTTATTTGGG AATCTCATTGCTTTGTCATTGGGTTTGACCCCTAATTTTAAATCAAATTTTGATTTGGCAGAAGATGCTAAATTACAGCTTTCAAA AGATACTTACGATGATGATTTAGATAATTTATTGGCGCAAATTGGAGATCAATATGCTGATTTGTTTTTGGCAGCTAAGAATTT ATCAGATGCTATTTTACTTTCAGATATCCTAAGAGTAAATACTGAAATAACTAAGGCTCCCCTATCAGCTTCAATGATTAAACGC TACGATGAACATCATCAAGACTTGACTCTTTTAAAAGCTTTAGTTCGACAACAACTTCCAGAAAAGTATAAAGAAATCTTTTTTG ATCAATCAAAAAACGGATATGCAGGTTATATTGATGGGGGAGCTAGCCAAGAAGAATTTTATAAATTTATCAAACCAATTTTAG AAAAAATGGATGGTACTGAGGAATTATTGGTGAAACTAAATCGTGAAGATTTGCTGCGCAAGCAACGGACCTTTGACAACGG CTCTATTCCCCATCAAATTCACTTGGGTGAGCTGCATGCTATTTTGAGAAGACAAGAAGACTTTTTATCCATTTTTAAAAGACAAT CGTGAGAAGATTGAAAAAATCTTGACTTTTCGAATTCCTTATTATGTTGGTCCATTGGCGCGTGGCAATAGTCGTTTTGCATGG ATGACTCGGAAGTCTGAAGAAACAATTACCCCATGGAATTTTGAAGAAGTTGTCGATAAAGGTGCTTCAGCTCAATCATTTATT GAACGCATGACAAACTTTGATAAAAATCTTCCAAATGAAAAAGTACTACCAAAACATAGTTTGCTTTATGAGTATTTTACGGTTT ATAACGAATTGACAAAGGTCAAATATGTTACTGAAGGAATGCGAAAACCAGCATTTCTTTTCAGGTGAACAGAAGAAAGCCATT GTTGATTTACTCTTCAAAACAAATCGAAAAGTAACCGTTAAGCAATTAAAAGAAGATTATTTCAAAAAAATAGAATGTTTTGAT AGTGTTGAAATTTCAGGAGTTGAAGATAGATTTAATGCTTCATTAGGTACCTACCATGATTTGCTAAAAATTATTAAAGATAAA GATTTTTTGGATAATGAAGAAAATGAAGATATCTTAGAGGATATTGTTTTAACATTGACCTTATTTGAAGATAGGGAGATGATT GAGGAAAGACTTAAAACATATGCTCACCTCTTTGATGATAAGGTGATGAAACAGCTTAAACGTCGCCGTTATACTGGTTGGGG ACGTTTGTCTCGAAAATTGATTAATGGTATTAGGGATAAGCAATCTGGCAAAACAATATTAGATTTTTTGAAATCAGATGGTTT TGCCAATCGCAATTTTATGCAGCTGATCCATGATGATAGTTTGACATTTAAAGAAGACATTCAAAAAGCACAAGTGTCTGGACA AGGCGATAGTTTACATGAACATATTGCAAATTTAGCTGGTAGCCCTGCTATTAAAAAAGGTATTTTACAGACTGTAAAAGTTGT TGATGAATTGGTCAAAGTAATGGGGCGGCATAAGCCAGAAAATATCGTTATTGAAATGGCACGTGAAAATCAGACAACTCAA AAGGGCCAGAAAAATTCGCGAGAGCGTATGAAACGAATCGAAGAAGGTATCAAAGAATTAGGAAGTCAGATTCTTAAAGAG CATCCTGTTGAAAATACTCAATTGCAAAATGAAAAGCTCTATCTCTATTATCTCCAAAATGGAAGAGACATGTATGTGGACCAA GAATTAGATATTAATCGTTTAAGTGATTATGATGTCGATCACATTGTTCCACAAAGTTTCCTTAAAGACGATTCAATAGACAATA AGGTCTTAACGCGTTCTGATAAAAATCGTGGTAAATCGGATAACGTTCCAAGTGAAGAAGTAGTCAAAAAGATGAAAAACTAT TGGAGACAACTTCTAAACGCCAAGTTAATCACTCAACGTAAGTTTGATAATTTAACGAAAGCTGAACGTGGAGGTTTGAGTGA ACTTGATAAAGCTGGTTTTATCAAACGCCAATTGGTTGAAACTCGCCAAATCACTAAGCATGTGGCACAAATTTTGGATAGTCG CATGAATACTAAATACGATGAAAATGATAAACTTATTCGAGAGGTTAAAGTGATTACCTTAAAATCTAAATTAGTTTCTGACTTC CGAAAAGATTTCCAATTCTATAAAGTACGTGAGATTAACAATTACCATCATGCCCATGATGCGTATCTAAATGCCGTCGTTGGA ACTGCTTTGATTAAGAAATATCCAAAACTTGAATCGGAGTTTGTCTATGGTGATTATAAAGTTTATGATGTTCGTAAAATGATTG CTAAGTCTGAGCAAGAAATAGGCAAAGCAACCGCAAAATATTTCTTTTACTCTAATATCATGAACTTCTTCAAAACAGAAATTA CACTTGCAAATGGAGAGATTCGCAAACGCCCTCTAATCGAAACTAATGGGGAAACTGGAGAAATTGTCTGGGATAAAGGGCG AGATTTTGCCACAGTGCGCAAAGTATTGTCCATGCCCCAAGTCAATATTGTCAAGAAAACAGAAGTACAGACAGGCGGATTCT CCAAGGAGTCAATTTTACCAAAAAGAAATTCGGACAAGCTTATTGCTCGTAAAAAAGACTGGGATCCAAAAAAATATGGTGGT TTTGATAGTCCAACGGTAGCTTATTCAGTCCTAGTGGTTGCTAAGGTGGAAAAAGGGAAATCGAAGAAGTTAAAATCCGTTAA AGAGTTACTAGGGATCACAATTATGGAAAGAAGTTCCTTTGAAAAAAATCCGATTGACTTTTTAGAAGCTAAAGGATATAAGG AAGTTAAAAAAGACTTAATCATTAAACTACCTAAATATAGTCTTTTTGAGTTAGAAAACGGTCGTAAACGGATGCTGGCTAGTG CCGGAGAATTACAAAAAGGAAATGAGCTGGCTCTGCCAAGCAAATATGTGAATTTTTTATATTTAGCTAGTCATTATGAAAAGT TGAAGGGTAGTCCAGAAGATAACGAACAAAAACAATTGTTTGTGGAGCAGCATAAGCATTATTTAGATGAGATTATTGAGCAA ATCAGTGAATTTTCTAAGCGTGTTATTTTAGCAGATGCCAATTTAGATAAAGTTCTTAGTGCATATAACAAACATAGAGACAAA 
CCAATACGTGAACAAGCAGAAAATATTATTCATTTATTTACGTTGACGAATCTTGGAGCTCCCGCTGCTTTTAAATATTTTGATA CAACAATTGATCGTAAACGATATACGTCTACAAAAGAAGTTTTAGATGCCACTCTTATCCATCAATCCATCACTGGTCTTTATGA AACACGCATTGATTTGAGTCAGCTAGGAGGTGACTGATAATCAAATGTCAGACGAAAATGCCAATTATTGAAGCGGCTAACGC CGCTTTTTTTGTTTCTGGTCTCCCTTGCTTGTACTTTACAGTATAGCTTCCTCAATGATCTCCTTATCCGG

$>$ pCas9g2-D15-KinA

ttaagatggcaagcttgacagccgaattcggattcccgcgTATATCTTGAATTAAAATTAAAATCTGCCAAGATCGAAAAATAAAAAGGATTT TTTGTGTCATTGGCGAATTATGATCTATTGAAGCAACCGTTAAGCTTACATAAGGAGGAACTACTATGGAACAGGATACGCAG CATGTTAAACCACTTCAAACAAAAACCGATATTCATGCAGTCTTGGCCTCTAATGGACGCATCATTTATATATCTGCCAACTCCA AACTGCATTTGGGCTATCTCCAAGGAGAGATGATCGGATCATTCCTCAAAACGTTTCTGCATGAGGAAGACCAATTTTTGGTTG AAAGCTATTTTTATAATGAACATCATCTGATGCCGTGCACCTTTCGTTTTATTAAAAAAGATCATACGATTGTGTGGGTGGAGG CTGCGGTAGAAATTGTTACGACAAGAGCTGAGCGGACAGAACGGGAAATCATTTTGAAAATGAAGGTTCTTGAAGAAGAAAC AGGCCATCAATCCCTAAACTGCGAAAAACATGAAATCGAACCTGCAAGCCCGGAATCGACTACATATATAACGGATGATTATG AACGGTTGGTTGAAAATCTCCCGAGTCCGCTATGCATCAGTGTCAAAGGCAAGATCGTCTATGTAAACAGCGCGATGCTTTCA ATGCTGGGAGCCAAAAGCAAGGATGCTATTATTGGTAAATCGTCCTATGAATTTATTGAAGAAGAATATCATGATATCGTGAA AAACAGGATTATACGAATGCAAAAAGGAATGGAAGTCGGAATGATTGAACAGACGTGGAAAAGGCTTGATGGCACACCTGTT CATTTAGAAGTGAAAGCATCCCCGACCGTCTACAAAAACCAGCAGGCTGAGCTGCTGCTGCTGATCGATATCTCTTCAAGGAA AAAATTCCAAACCATCCTGCAAAAAAGCCGTGAACGATATCAGCTGCTGATTCAAAATTCCATTGATACCATTGCGGTGATTCA CAATGGAAAATGGGTATTTATGAATGAATCGGGAATTTCCCTGTTTGAAGCGGCTACATATGAGGATTTAATTGGCAAAAACA TATACGATCAGCTGCATCCTTGCGATCACGAGGATGTAAAAGAGAGAATCCAAAACATTGCCGAGCAAAAAACAGAATCTGAA ATTGTCAAGCAATCCTGGTTCACCTTTCAGAACAGGGTCATCTATACGGAGATGGTCTGCATTCCGACGACCTTTTTTGGTGAA GCGGCCGTCCAGGTCATTCTTCGGGACATCTCAGAGAGAAAACAAACAGAAGAATTGATGCTGAAATCGGAAAAATTATCAAT CGCAGGGCAGCTCGCGGCGGGAATCGCCCATGAGATCCGCAACCCTCTTACAGCGATCAAAGGATTTTTACAGCTGATGAAAC CGACAATGGAAGGCAACGAACATTACTTTGATATTGTGTTTTCTGAACTCAGCCGTATCGAATTAATACTCAGTGAACTGCTCA TGCTGGCGAAACCTCAGCAAAATGCTGTCAAAGAATATTTGAACTTGAAAAAATTAATTGGTGAGGTTTCAGCCCTGTTAGAA ACGCAGGCGAATTTAAATGGCATTTTTATCAGAACAAGTTATGAAAAAGACAGCATTTATATAAACGGGGATCAAAACCAATT AAAGCAGGTATTCATTAATTTAATCAAAAATGCAGTTGAATCAATGCCTGATGGGGGAACAGTAGACATTATCATAACCGAAG ATGAGCATTCTGTTCATGTTACTGTCAAAGACGAAGGGGAAGGTATACCTGAAAAGGTACTAAACCGGATTGGAGAGCCATTT TTAACAACAAAAGAAAAAGGTACGGGGCTTGGATTAATGGTGACATTTAATATCATTGAAAACCATCAGGGAGTTATACATGT GGACAGCCATCCTGAAAAAGGCACAGCGTTTAAAATTTCATTTCcaaaaaaataagcatgcccggggatggacgagctttacaaGTAATCAA ATGTCAGACGAAAATGCCAATTATTGAAGCGGCTAACGCCGCTTTTTTTGTTTCTGGTCTCCCAACTTTGCTAAAGAGCGTCTCA GCTTCTAGAGTAGAGATCTGCAGTGACTGGAGCTTCTAGAGATCAGGTCAGACGCGATTTCCTGGGTGCTCGAGCCATGGGA AAGCGGTGGTGGAAAAAAGCCGAATGAAGCGGATGAAATTGCTCATCGTCTCGTGAAAAAGCCGAAGAAGGTTAAGCCTG GGTATAAAAAGAAAATGAGCTATGAGATGGAGAAATTGAAGTCATGCGCCGGTTAGTTTTAGAGCTAGAAATAGCAAGTTAA AATAAGGCTAGTCCGTTATCAACTTGAAAAAGTGGCACCGAGTCGGTGCTTTTTTTCTCGGTACCAAATTCCAGAAAAGAGGCC TCCCGAAAGGGGGGCCTTTTTTCGTTTTGGTCCCTCAATGATCCGACAGGAAGTGGTATTAGCGACTCCTACAAGGAATAGGT GTCTGGGTCATGCAGTGACTGGGAAAACCCTGGCGACTAGTCTTGGACTCCTGTTGATAGATCCAGTAATGACCTCAGAACTC CATCTGGATTTGTTCAGAACGCTCGGTTGCCGCCGGGCGTTTTTTATTGGTGAGAATCCAGGGGTCCCCAATAATTACGATTTA AATCCTTCAAACTTCCCAAAGGCGAGCCCTAGTGACATTAGAAAACCGACTGTAAAAAGTACAGTCGGCATTATCTCATATTAT AAAAGCCAGTCATTAGGCCTATCTGACAATTCCTGAATAGAGTTCATAAACAATCCTGCATGATAACCATCACAAACAGAATGA TGTACCTGTAAAGATAGCGGTAAATATATTGAATTACCTTTATTAATGAATTTTCCTGCTGTAATAATGGGTAGAAGGTAATTAC TATTATTATTGATATTTAAGTTAAACCCAGTAAATGAAGTCCATGGAATAATAGAAAGAGAAAAAGCATTTTCAGGTATAGGTG TTTTGGGAAACAATTTCCCCGAACCATTATATTTCTCTACATCAGAAAGGTATAAATCATAAAACTCTTTGAAGTCATTCTTTACA GGAGTCCAAATACCAGAGAATGTTTTAGATACACCATCAAAAATTGTATAAAGTGGCTCTAACTTATCCCAATAACCTAACTCTC CGTCGCTATTGTAACCAGTTCTAAAAGCTGTATTTGAGTTTATCACCCTTGTCACTAAGAAAATAAATGCAGGGTAAAATTTATA TCCTTCTTGTTTTATGTTTCGGTATAAAACACTAATATCAATTTCTGTGGTTATACTAAAAGTCGTTTGTTGGTTCAAATAATGAT TAAATATCTCTTTTCTCTTCCAATTGTCTAAATCAATTTTATTAAAGTTCATTTGATATGCCTCCTAAATTTTTATCTAAAGTGAAT TTAGGAGGCTTACTTGTCTGCTTTCTTCATTAGAATCAATCCTTTTTTAAAAGTCAATCCCGTTTGTTGAAGACTTTTGTCCTTTTC CGCTGCATAAACAATATGGCCCGTTTGTTGAACTACTCTTTAATAAAATAATTTTTCCGTTCCCAATTCCACATTGCAATAATAGA AAATCCATCTTCATCGGCTTTTTCGTCATCATCTGTATGAATCAAATCGCCTTCTTCTGTGTCATCAAGGTTTAATTTTTTATGTAT TTCTTTTAACAAACCACCATAGGAGATTAACCTTTTACGGTGTAAACCTTCCTCCAAATCAGACAAACGTTTCAAATTCTTTTCTT CATCATCGGTCATAAAATCCGTATCCTTTACAGGATATTTTGCAGTTTCGTCAATTGCCGATTGTATATCCGATTTATATTTATTT TTCGGTCGAATCATTTGAACTTTTACATTTGGATCATAGTCTAATTTCATTGCCTTTTTCCAAAATTGAATCCATTGTTTTTGATTC 
ACGTAGTTTTCTGTATTCTTAAAATAAGTTGGTTCCACACATACCAATACATGCATGTGCTGATTATAAGAATTATCTTTATTATT TATTGTCACTTCCGTTGCACGCATAAAACCAACAAGATTTTTATTAATTTTTTTATATTGCATCATTCGGCGAAATCCTTGAGCCA TATCTGACAAACTCTTATTTAATTCTTCGCCATCATAAACATTTTTAACTGTTAATGTGAGAAACAACCAACGAACTGTTGGCTTT TGTTTAATAACTTCAGCAACAACCTTTTGTGACTGAATGCCATGTTTCATTGCTCTCCTCCAGTTGCACATTGGACAAAGCCTGG ATTTACAAAACCACACTCGATACAACTTTCTTTCGCCTGTTTCACGATTTTGTTTATACTCTAATATTTCAGCACAATCTTTTACTC TTTCAGCCTTTTTAAATTCAAGAATATGCAGAAGTTCAAAGTAATCAACATTAGCGATTTTCTTTTCTCTCCATGGTCTCACTTTT CCACTTTTTGTCTTGTCCACTAAAACCCTTGATTTTTCATCTGAATAAATGCTACTATTAGGACACATAATATTAAAAGAAACCCC CATCTATTTAGTTATTTGTTTGGTCACTTATAACTTTAACAGATGGGGTTTTTCTGTGCAACCAATTTTAAGGGTTTTCCAATACT TTAAAACACATACATACCAACACTTCAACGCACCTTTCAGCAACTAAAATAAAAATGACGTTATTTCTATATGTATCAAGATAAG AAAGAACAAGTTCAAAACCATCAAAAAAAGACACCTTTTCAGGTGCTTTTTTTATTTTATAAACTCATTCCCTGATCTCGACTTC GTTCTTTTTTTTACCTCTCGGTTATGAGTTAGTTCAAATTCGTTCTTTTTAAGGTTCTAAATCGTGTTTTTCTTGGAATTGTGCTGTTT TATCCTTTACCTTGTCTACAAACCCCTTAAAAACGTTTTTAAAGGCTTTTAAGCGTCTGTACGTTCCTTAAGGAATTATTCCTTAG TGCTTTCTAGGTTAATGTCATGATAATAATGGTTTCTTAGACGTCAGGTGGCACTTTTTCGGGGAAATGTCCGCGGAACCCCTAT TTGTTTATTTTTCTAAATACATTCAAATATGTATCCGCTCATGAGACAATAACCCTGATAAATGCTTCAATAATATTGAAAAAGG AAGAGTATGAGTATTCAACATTTCCGTGTCGCCCTTATTCCCTTTTTTGCGGCATTTTGCCTTCCTGTTTTTGCTCACCCAGAAAC GCTGGTGAAAGTAAAAGATGCTGAAGATCAGTTGGGTGCACGAGTGGGTTACATCGAACTGGATCTCAACAGCGGTAAGATC CTTGAGAGTTTTCGCCCCGAAGAACGTTTTCCAATGATGAGCACTTTTAAAGTTCTGCTATGTGGCGCGGTATTATCCCGTGTT GACGCCGGGCAAGAGCAACTCGGTCGCCGCATACACTATTCTCAGAATGACTTGGTTGAGTACTCACCAGTCACAGAAAAGCA TCTTACGGATGGCATGACAGTAAGAGAATTATGCAGTGCTGCCATAACCATGAGTGATAACACTGCGGCCAACTTACTTCTGA CAACGATCGGAGGACCGAAGGAGCTAACCGCTTTTTTGCACAACATGGGGGATCATGTAACTCGCCTTGATCGTTGGGAACCG GAGCTGAATGAAGCCATACCAAACGACGAGCGTGACACCACGATGCCTGCAGCAATGGCAACAACGTTGCGCAAACTATTAA CTGGCGAACTACTTACTCTAGCTTCCCGGCAACAATTAATAGACTGGATGGAGGCGGATAAAGTTGCAGGACCACTTCTGCGC TCGGCCCTTCCGGCTGGCTGGTTTATTGCTGATAAATCTGGAGCCGGTGAGCGTGGGTCTCGCGGTATCATTGCAGCACTGGG GCCAGATGGTAAGCCCTCCCGTATCGTAGTTATCTACACGACGGGGAGTCAGGCAACTATGGATGAACGAAATAGACAGATC GCTGAGATAGGTGCCTCACTGATTAAGCATTGGTAACTGTCAGACCAAGTTTACTCATATATACTTTAGATTGATTTAAAACTTC ATTTTTAATTTAAAAGGATCTAGGTGAAGATCCTTTTTGATAATCTCATGACCAAAATCCCTTAACGTGAGTTTTCGTTCCACTG AGCGTCAGACCCCTTAATAAGATGATCTTCTTGAGATCGTTTTGGTCTGCGCGTAATCTCTTGCTCTGAAAACGAAAAAACCGC CTTGCAGGGAGGTTTTTCGAAGGTTCTCTGAGCTACCAACTCTTTGAACCGAGGTAACTGGCTTGCAGGAGCGCAGTCACCAA AACTTGTCCTTTCAGTTTAGCCTTAACCGGCGCATGACTTCAAGACTAACTCCTCTAAATCAATTACCAGTGGCTGCTGCCAGTG GTGCTTTTGCATGTCTTTCCGGGTTGGACTCAAGACGATAGTTACCGGATAAGGCGCAGCGGTCGGACTGAACGGGGGGTTC GTGCATACAGTCCAGCTTGGAGCGAACTGCCTACCCGGAACTGAGTGTCAGGCGTGGAATGAGACAAACGCGGCCATAACAG CGGAATGACACCGGTAAACCGAAAGGCAGGAACAGGAGAGCGCACGAGGGAGCCGCCAGGGGGAAACGCCTGGTATCTTTA TAGTCCTGTCGGGTTTCGCCACCACTGATTTGAGCGTCAGATTTCGTGATGCTTGTCAGGGGGCGGAGCCTATGGAAAAACGC TTTGCCCGAGCCTATGGAAAAACGCTTTGCCCCACACAGGCTCTTGAACAAGGGATAACAATTTGTCATAGCTGTATTCGTTAC AAGGAGGATTTTCGAAAAAACACCCTAACGGGTGTTTTTTTATAGCTGGTCTCCCCAAGTGCTGTAAAAAACGCCGAATGAAG CGGATGAAATTGCTCATCGTCTCGTGAAAAAGCCGAAGAAGGTTAAGCCTGGGTATAAAAAGAAAATGAGCTATGAGATGGA GAAAGCCATAACCATGAGTGATAACACTGGTTTTAGAGCTAGAAATAGCAAGTTAAAATAAGGCTAGTCCGTTATCAACTTGA AAAAGTGGCACCGAGTCGGTGCTTTTTTTCTCGGTACCAAATTCCAGAAAAGAGGCCTCCCGAAAGGGGGGCCTTTTTTCGTTT TGGTCCCTCAATGATCTCCTTATCCGGTTAAGATGGCTTTTAAATCAATTTTCAGCTCCTGTATACAATTACCAAAGTTTTTCTGA ATGAAGCCATGTGTTTTGACACATTCTATACTCACAAGGAGGTGAGACACATGAGTAAATCCTGGGGCAAATTCATCGAGGAG GAAGAGGCGGAAATGGCTTCCCGTCGTAATCTAATGATTGTCGATGGAACTAACTTAGGCTTTCGCTTCAAACATAACAATAGT AAAAAACCATTTGCCTCAAGTTATGTTTCAACTATTCAATCTCTGGCAAAATCCTACTCTGCCAGAACTACGATTGTTCTAGGTG ATAAGGGCAAAAGCGTGTTCAGACTAGAACATCTACCAGAGTATAAAGGTAATCGTGATGAAAAGTACGCACAACGTACGGA AGAGGAGAAAGCGCTAGATGAGCAGTTCTTCGAATACCTCAAAGATGCTTTCGAGTTGTGTAAAACTACATTCCCAACTTTTAC CATTCGTGGTGTAGAAGCAGATGACATGGCGGCGTATATTGTTAAGCTCATCGGGCATCTTTATGATCACGTTTGGCTAATATC TACAGATGGTGACTGGGATACTTTATTAACGGATAAAGTTTCTCGTTTTTCTTTCACAACACGTCGTGAGTATCATCTTCGTGAT ATGTATGAGCACCATAACGTGGACGACGTAGAACAGTTTATCTCCCTGAAAGCAATTATGGGAGATCTAGGAGATAATATTCG TGGTGTTGAAGGAATAGGAGCAAAACGCGGATATAATATTATTCGTGAGTTTGGTAACGTACTGGATATTATTGATCAGCTTC CACTGCCTGGAAAGCAGAAATATATACAGAACCTGAATGCATCGGAAGAACTGCTTTTCCGAAACTTGATTCTGGTTGATTTAC CTACCTACTGTGTGGATGCTATTGCTGCTGTAGGTCAAGATGTGTTAGATAAGTTCACGAAAGACATCCTTGAGATCGCGGAA CAATGATAAGCTCTTAAAGGGGGTTTTAGATACCAGAGATGGATAAGAAATACTCAATAGGCTTAGATATCGGCACAAATAGC GTCGGATGGGCGGTGATCACTGATGAATATAAGGTTCCGTCTAAAAAGTTCAAGGTTCTGGGAAATACAGACCGCCACAGTAT CAAAAAAAATCTTATAGGGGCTCTTTTATTTGACAGTGGAGAGACAGCGGAAGCGACTCGTCTCAAACGGACAGCTCGTAGAA 
GGTATACACGTCGGAAGAATCGTATTTGTTATCTACAGGAGATTTTTTCAAATGAGATGGCGAAAGTAGATGATAGTTTCTTTC ATCGACTTGAAGAGTCTTTTTTGGTGGAAGAAGACAAGAAGCATGAACGTCATCCTATTTTTGGAAATATAGTAGATGAAGTT GCTTATCATGAGAAATATCCAACTATCTATCATCTGCGAAAAAAATTGGTAGATTCTACTGATAAAGCGGATTTGCGCTTAATCT ATTTGGCCTTAGCGCATATGATTAAGTTTCGTGGTCATTTTTTGATTGAGGGAGATTTAAATCCTGATAATAGTGATGTGGACA AACTATTTATCCAGTTGGTACAAACCTACAATCAATTATTTGAAGAAAACCCTATTAACGCAAGTGGAGTAGATGCTAAAGCGA TTCTTTCTGCACGATTGAGTAAATCAAGACGATTAGAAAATCTCATTGCTCAGCTCCCCGGTGAGAAGAAAAATGGCTTATTTG GGAATCTCATTGCTTTGTCATTGGGTTTGACCCCTAATTTTAAATCAAATTTTGATTTGGCAGAAGATGCTAAATTACAGCTTTC AAAAGATACTTACGATGATGATTTAGATAATTTATTGGCGCAAATTGGAGATCAATATGCTGATTTGTTTTTGGCAGCTAAGAA TTTATCAGATGCTATTTTACTTTCAGATATCCTAAGAGTAAATACTGAAATAACTAAGGCTCCCCTATCAGCTTCAATGATTAAA CGCTACGATGAACATCATCAAGACTTGACTCTTTTAAAAGCTTTAGTTCGACAACAACTTCCAGAAAAGTATAAAGAAATCTTTT TTGATCAATCAAAAAACGGATATGCAGGTTATATTGATGGGGGAGCTAGCCAAGAAGAATTTTATAAATTTATCAAACCAATTT TAGAAAAAATGGATGGTACTGAGGAATTATTGGTGAAACTAAATCGTGAAGATTTGCTGCGCAAGCAACGGACCTTTGACAAC GGCTCTATTCCCCATCAAATTCACTTGGGTGAGCTGCATGCTATTTTGAGAAGACAAGAAGACTTTTATCCATTTTTAAAAGACA ATCGTGAGAAGATTGAAAAAATCTTGACTTTTCGAATTCCTTATTATGTTGGTCCATTGGCGCGTGGCAATAGTCGTTTTGCAT GGATGACTCGGAAGTCTGAAGAAACAATTACCCCATGGAATTTTGAAGAAGTTGTCGATAAAGGTGCTTCAGCTCAATCATTT ATTGAACGCATGACAAACTTTGATAAAAATCTTCCAAATGAAAAAGTACTACCAAAACATAGTTTGCTTTATGAGTATTTTACG GTTTATAACGAATTGACAAAGGTCAAATATGTTACTGAAGGAATGCGAAAACCAGCATTTCTTTCAGGTGAACAGAAGAAAGC CATTGTTGATTTACTCTTCAAAACAAATCGAAAAGTAACCGTTAAGCAATTAAAAGAAGATTATTTCAAAAAAATAGAATGTTTT GATAGTGTTGAAATTTCAGGAGTTGAAGATAGATTTAATGCTTCATTAGGTACCTACCATGATTTGCTAAAAATTATTAAAGAT AAAGATTTTTTGGATAATGAAGAAAATGAAGATATCTTAGAGGATATTGTTTTAACATTGACCTTATTTGAAGATAGGGAGATG ATTGAGGAAAGACTTAAAACATATGCTCACCTCTTTGATGATAAGGTGATGAAACAGCTTAAACGTCGCCGTTATACTGGTTGG GGACGTTTGTCTCGAAAATTGATTAATGGTATTAGGGATAAGCAATCTGGCAAAACAATATTAGATTTTTTGAAATCAGATGGT TTTGCCAATCGCAATTTTATGCAGCTGATCCATGATGATAGTTTGACATTTAAAGAAGACATTCAAAAAGCACAAGTGTCTGGA CAAGGCGATAGTTTACATGAACATATTGCAAATTTAGCTGGTAGCCCTGCTATTAAAAAAGGTATTTTACAGACTGTAAAAGTT GTTGATGAATTGGTCAAAGTAATGGGGCGGCATAAGCCAGAAAATATCGTTATTGAAATGGCACGTGAAAATCAGACAACTC AAAAGGGCCAGAAAAATTCGCGAGAGCGTATGAAACGAATCGAAGAAGGTATCAAAGAATTAGGAAGTCAGATTCTTAAAG AGCATCCTGTTGAAAATACTCAATTGCAAAATGAAAAGCTCTATCTCTATTATCTCCAAAATGGAAGAGACATGTATGTGGACC AAGAATTAGATATTAATCGTTTAAGTGATTATGATGTCGATCACATTGTTCCACAAAGTTTCCTTAAAGACGATTCAATAGACAA TAAGGTCTTAACGCGTTCTGATAAAAATCGTGGTAAATCGGATAACGTTCCAAGTGAAGAAGTAGTCAAAAAGATGAAAAACT ATTGGAGACAACTTCTAAACGCCAAGTTAATCACTCAACGTAAGTTTGATAATTTAACGAAAGCTGAACGTGGAGGTTTGAGT GAACTTGATAAAGCTGGTTTTATCAAACGCCAATTGGTTGAAACTCGCCAAATCACTAAGCATGTGGCACAAATTTTGGATAGT CGCATGAATACTAAATACGATGAAAATGATAAACTTATTCGAGAGGTTAAAGTGATTACCTTAAAATCTAAATTAGTTTCTGAC TTCCGAAAAGATTTCCAATTCTATAAAGTACGTGAGATTAACAATTACCATCATGCCCATGATGCGTATCTAAATGCCGTCGTTG GAACTGCTTTGATTAAGAAATATCCAAAACTTGAATCGGAGTTTGTCTATGGTGATTATAAAGTTTATGATGTTCGTAAAATGA TTGCTAAGTCTGAGCAAGAAATAGGCAAAGCAACCGCAAAATATTTCTTTTACTCTAATATCATGAACTTCTTCAAAACAGAAA TTACACTTGCAAATGGAGAGATTCGCAAACGCCCTCTAATCGAAACTAATGGGGAAACTGGAGAAATTGTCTGGGATAAAGG GCGAGATTTTGCCACAGTGCGCAAAGTATTGTCCATGCCCCAAGTCAATATTGTCAAGAAAACAGAAGTACAGACAGGCGGAT TCTCCAAGGAGTCAATTTTACCAAAAAGAAATTCGGACAAGCTTATTGCTCGTAAAAAAGACTGGGATCCAAAAAAATATGGT GGTTTTGATAGTCCAACGGTAGCTTATTCAGTCCTAGTGGTTGCTAAGGTGGAAAAAGGGAAATCGAAGAAGTTAAAATCCGT TAAAGAGTTACTAGGGATCACAATTATGGAAAGAAGTTCCTTTGAAAAAAATCCGATTGACTTTTTAGAAGCTAAAGGATATA AGGAAGTTAAAAAAGACTTAATCATTAAACTACCTAAATATAGTCTTTTTTGAGTTAGAAAACGGTCGTAAACGGATGCTGGCTA GTGCCGGAGAATTACAAAAAGGAAATGAGCTGGCTCTGCCAAGCAAATATGTGAATTTTTTATATTTAGCTAGTCATTATGAAA AGTTGAAGGGTAGTCCAGAAGATAACGAACAAAAACAATTGTTTGTGGAGCAGCATAAGCATTATTTAGATGAGATTATTGAG CAAATCAGTGAATTTTCTAAGCGTGTTATTTTAGCAGATGCCAATTTAGATAAAGTTCTTAGTGCATATAACAAACATAGAGAC AAACCAATACGTGAACAAGCAGAAAATATTATTCATTTATTTACGTTGACGAATCTTGGAGCTCCCGCTGCTTTTAAATATTTTG ATACAACAATTGATCGTAAACGATATACGTCTACAAAAGAAGTTTTAGATGCCACTCTTATCCATCAATCCATCACTGGTCTTTA TGAAACACGCATTGATTTGAGTCAGCTAGGAGGTGACTGATAATCAAATGTCAGACGAAAATGCCAATTATTGAAGCGGCTAA CGCCGCTTTTTTTGTTTCTGGTCTCCCTTGCTTGTACTTTACAGTATAGCTTCCTCAATGATCTCCTTATCCGG 